\title{
Phosphine-Mediated MBH-Type/Acyl Transfer/Wittig Sequence for Construction of Functionalized Furo[3,2-c]coumarins
}

Sandip Sambhaji Vagh, Bo-Jhih Hou, Athukuri Edukondalu, Pin-Ching Wang, and Wenwei Lin* Department of Chemistry, National Taiwan Normal University, 88, Sec. 4, Tingchow Road, Taipei 11677, Taiwan R.O.C.

\section{Table of Contents}

$\begin{array}{ll}\text { I. General information } & \text { S2-S2 }\end{array}$

II. Reaction optimization

III. Control experiments

IV. Experimental procedures

a. Typical procedure (TP-1) for the preparation of alkynoates $\mathbf{1 / 9}$

b. Typical procedure (TP-2) for the preparation of functionalized furo[3,2-c]coumarins 4

c. Typical procedure (TP-3) for the preparation of 2,3-disubstituted furo[3,2-c]coumarins 6

d. Typical procedure (TP-4) for the preparation of 2,3-disubstituted furo[3,2-c]quinolinones 7 


\section{General Information}

All reactions were carried out under argon atmosphere in oven-dried glassware with magnetic stirring. Unless otherwise stated, all reagents were used as purchased from commercial suppliers without further purification. 1,2-Dichloroethane (DCE) was freshly distilled from calcium hydride under argon atmosphere. Yields refer to isolated yields of compounds estimated to be $>95 \%$ pure as determined by ${ }^{1} \mathrm{H}$ NMR. Analytical thin layer chromatography (TLC) was performed on pre coated alumina-backed silica gel plates (Merck 60 F254, $0.2 \mathrm{~mm}$ thickness) which were developed using UV irradiation at $254 \mathrm{~nm}$. Flash column chromatography was performed using silica gel (SiliCycle SiliaFlash P60, 230-400 mesh). Melting points were measured on a hostage melting point apparatus and are uncorrected. IR spectra were recorded on a Perkin Elmer 500 spectrometer and only selected peaks are mentioned. ${ }^{1} \mathrm{H}$ NMR spectra were recorded on either an Oxford JEOL $400 \mathrm{MHz}$ spectrometer or a Bruker Ascend $400 \mathrm{MHz}$ spectrometer. ${ }^{13} \mathrm{C}$ NMR spectra at $100 \mathrm{MHz},{ }^{31} \mathrm{P}$ NMR at 162 $\mathrm{MHz}$ and ${ }^{19} \mathrm{~F}$ NMR at $376 \mathrm{MHz}$. Chemical shifts are reported in $\delta \mathrm{ppm}$ referenced to an internal TMS standard $(\delta=0.0 \mathrm{ppm})$ for ${ }^{1} \mathrm{H} \mathrm{NMR}, \mathrm{CDCl} 3(\delta=77.0 \mathrm{ppm})$ for $\mathrm{CD}_{3} \mathrm{OD}(\delta 3.31 \mathrm{ppm}$ for ${ }^{1} \mathrm{H}$ NMR and $49.0 \mathrm{ppm}$ for $\left.{ }^{13} \mathrm{C} \mathrm{NMR}\right), \mathrm{H}_{3} \mathrm{PO}_{4}(\delta=0.0 \mathrm{ppm})$ for ${ }^{31} \mathrm{P} \mathrm{NMR}$, and $\mathrm{C}_{6} \mathrm{H}_{5} \mathrm{~F}(\delta=-$ $113.15 \mathrm{ppm}$ ) for ${ }^{19} \mathrm{~F}$ NMR. The following abbreviations (or combinations thereof) were used to explain the multiplicities: $\mathrm{s}=$ singlet, $\mathrm{d}=$ doublet, $\mathrm{t}=$ triplet, $\mathrm{q}=$ quartet, $\mathrm{m}=$ multiplet, $\mathrm{dd}$ $=$ doublet of doublet, $\mathrm{dt}=$ doublet of triplet, $\mathrm{td}=$ triplet of doublet, $\mathrm{tt}=$ triplet of triplet, $\mathrm{qd}=$ quartet of doublet, $\mathrm{br}=$ broad, $\mathrm{p}=$ pseudo. High resolution mass spectra were recorded on Waters XeVo G2-S QTof using ESI (TOF analyzer). The X-ray diffraction measurements were carried out at $200 \mathrm{~K}$ on either a Bruker D8 Venture or a Bruker KAPPA APEX II CCD area detector system equipped with a graphite monochromator and a Mo-K $\alpha$ fine-focus sealed tube $(\mathrm{k}=0.71073 \AA)$. 


\section{Reaction Optimization:}

\section{Table S1. Optimization of Reaction Conditions for 4aa}

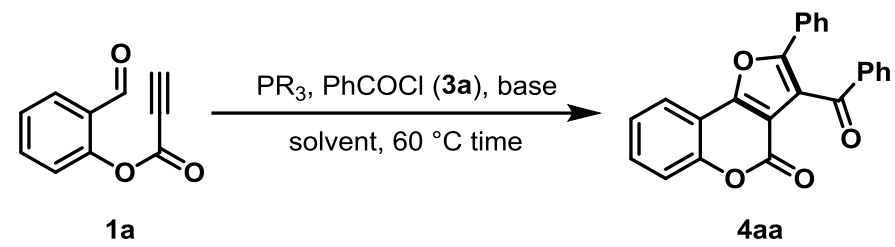

\begin{tabular}{|c|c|c|c|c|c|}
\hline entry & $\mathrm{PR}_{3}$ & base & solvent & $t(\mathrm{~h})$ & $4 \mathbf{a a}^{b}$ \\
\hline $1^{c, d}$ & $\mathrm{PPh}_{3}$ & $\mathrm{Et}_{3} \mathrm{~N}$ & $\mathrm{CH}_{3} \mathrm{CN}$ & 12 & 22 \\
\hline $2^{c}$ & $\mathrm{PPh}_{3}$ & $\mathrm{Et}_{3} \mathrm{~N}$ & $\mathrm{CH}_{3} \mathrm{CN}$ & 6 & 29 \\
\hline 3 & $\mathrm{PPh}_{3}$ & $\mathrm{Et}_{3} \mathrm{~N}$ & $\mathrm{CH}_{3} \mathrm{CN}$ & 8 & 60 \\
\hline 4 & $\mathrm{PBu}_{3}$ & $\mathrm{Et}_{3} \mathrm{~N}$ & $\mathrm{CH}_{3} \mathrm{CN}$ & 2 & 7 \\
\hline 5 & $\mathrm{MePPh}_{2}$ & $\mathrm{Et}_{3} \mathrm{~N}$ & $\mathrm{CH}_{3} \mathrm{CN}$ & 2 & 66 \\
\hline 6 & $\mathrm{Me}_{2} \mathrm{PPh}$ & $\mathrm{Et}_{3} \mathrm{~N}$ & $\mathrm{CH}_{3} \mathrm{CN}$ & 2 & 40 \\
\hline 7 & $\mathrm{EtPPh}_{2}$ & $\mathbf{E t}_{3} \mathbf{N}$ & $\mathrm{CH}_{3} \mathrm{CN}$ & 4 & 80 \\
\hline 8 & $\mathrm{Et}_{2} \mathrm{PPh}$ & $\mathrm{Et}_{3} \mathrm{~N}$ & $\mathrm{CH}_{3} \mathrm{CN}$ & 2 & 50 \\
\hline 9 & $\mathrm{P}(\mathrm{Cy})_{3}$ & $\mathrm{Et}_{3} \mathrm{~N}$ & $\mathrm{CH}_{3} \mathrm{CN}$ & 2 & 16 \\
\hline 10 & $\mathrm{EtPPh}_{2}$ & $\mathrm{Et}_{3} \mathrm{~N}$ & THF & 2 & 74 \\
\hline 11 & $\mathrm{EtPPh}_{2}$ & $\mathrm{Et}_{3} \mathrm{~N}$ & toluene & 1 & 75 \\
\hline 12 & $\mathrm{EtPPh}_{2}$ & $\mathrm{Et}_{3} \mathrm{~N}$ & EtOAc & 1 & 71 \\
\hline 13 & $\mathrm{EtPPh}_{2}$ & $\mathrm{Et}_{3} \mathrm{~N}$ & DCE & 1 & 61 \\
\hline 14 & $\mathrm{EtPPh}_{2}$ & DIPEA & $\mathrm{CH}_{3} \mathrm{CN}$ & 1 & 76 \\
\hline 15 & $\mathrm{EtPPh}_{2}$ & DBU & $\mathrm{CH}_{3} \mathrm{CN}$ & 1 & 70 \\
\hline 16 & $\mathrm{EtPPh}_{2}$ & TMG & $\mathrm{CH}_{3} \mathrm{CN}$ & 2 & 59 \\
\hline 17 & $\mathrm{EtPPh}_{2}$ & pyridine & $\mathrm{CH}_{3} \mathrm{CN}$ & 2 & 56 \\
\hline $18^{e}$ & $\mathrm{EtPPh}_{2}$ & $\mathrm{Et}_{3} \mathrm{~N}$ & $\mathrm{CH}_{3} \mathrm{CN}$ & 3 & 67 \\
\hline $19^{f}$ & $\mathrm{EtPPh}_{2}$ & $\mathrm{Et}_{3} \mathrm{~N}$ & $\mathrm{CH}_{3} \mathrm{CN}$ & 1 & 77 \\
\hline
\end{tabular}

${ }^{a}$ The reactions were carried out with compound $1 \mathbf{a}(0.1 \mathrm{mmol}), \mathrm{PR}_{3}$ (1.1 equiv), $\mathrm{PhCOCl}$ (3a) (2.5 equiv) and base $\left(1.5\right.$ equiv) in dry solvent $(1.0 \mathrm{~mL})$ under argon atmosphere at $60{ }^{\circ} \mathrm{C} .{ }^{b}$ Yield of the product $4 \mathbf{a a}$ was determined by NMR analysis of the crude reaction mixture using 1,3,5- trimethoxybenzene as an internal standard. ${ }^{c} 1.2$ equiv of $\mathrm{PhCOCl}$ (3a) was used. ${ }^{d}$ Reaction carried out at $30{ }^{\circ} \mathrm{C}$. ${ }^{e}$ Reaction carried out at $40{ }^{\circ} \mathrm{C} .{ }^{f}$ Reaction carried out at $100{ }^{\circ} \mathrm{C}$. DCE $=1,2$-Dichloroethane. 
Table S2. Optimization of Reaction Conditions for 4aa by Using $\mathbf{P P h}_{3}{ }^{a}$

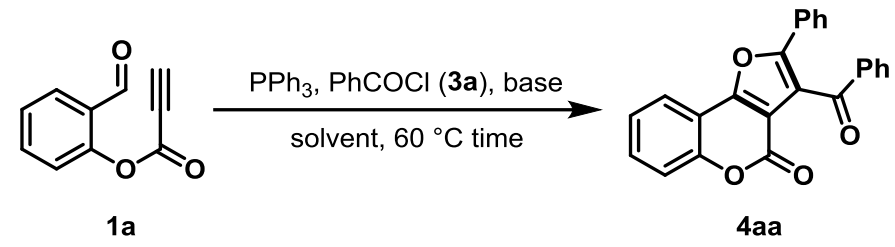

\begin{tabular}{|c|c|c|c|c|}
\hline entry & base & solvent & $t(\mathrm{~h})$ & $\mathbf{4 a a}^{b}$ \\
\hline 1 & $\mathrm{Et}_{3} \mathrm{~N}$ & $\mathrm{CH}_{3} \mathrm{CN}$ & 8 & 60 \\
\hline 2 & $\mathrm{Et}_{3} \mathrm{~N}$ & THF & 6 & 52 \\
\hline 3 & $\mathrm{Et}_{3} \mathrm{~N}$ & toluene & 3 & 60 \\
\hline 4 & $\mathrm{Et}_{3} \mathrm{~N}$ & EtOAc & 3 & 47 \\
\hline 5 & $\mathrm{Et}_{3} \mathrm{~N}$ & DCE & 3 & 76 \\
\hline 6 & DIPEA & DCE & 3 & 80 \\
\hline 7 & DBU & DCE & 3 & 70 \\
\hline 8 & TMG & DCE & 3 & 55 \\
\hline 9 & pyridine & DCE & 3 & 34 \\
\hline $10^{c}$ & DIPEA & DCE & 3 & 62 \\
\hline $11^{d}$ & DIPEA & DCE & 2 & 77 \\
\hline $12^{e}$ & DIPEA & DCE & 3 & 80 \\
\hline $13^{f}$ & DIPEA & DCE & 3 & 77 \\
\hline $14^{g}$ & DIPEA & DCE & 3 & 78 \\
\hline $15^{h}$ & DIPEA & DCE & 6 & 80 \\
\hline $16^{i}$ & DIPEA & DCE & 3 & 80 \\
\hline
\end{tabular}

${ }^{a}$ The reactions were carried out with compound $1 \mathbf{a}(0.1 \mathrm{mmol}), \mathrm{PPh}_{3}$ (1.1 equiv), $\mathrm{PhCOCl}$ (3a) (2.5 equiv) and base $\left(1.5\right.$ equiv) in dry solvent $(1.0 \mathrm{~mL})$ under argon atmosphere at $60{ }^{\circ} \mathrm{C} .{ }^{b}$ Yield of the product $4 \mathbf{a a}$ was determined by NMR analysis of the crude reaction mixture using 1,3,5-trimethoxybenzene as an internal standard. ${ }^{c}$ Reaction carried out at $40{ }^{\circ} \mathrm{C}$. ${ }^{d}$ Reaction carried out at $80{ }^{\circ} \mathrm{C}$. ${ }^{e} 2.0$ equiv of DIPEA was used. ${ }^{f}$ Addition sequence changed to $\mathrm{PPh}_{3}$, base and benzoyl chloride. ${ }^{g} 0.5 \mathrm{~mL}$ DCE was used. ${ }^{h} 1.5 \mathrm{~mL}$ DCE was used. ${ }^{i 2} .2$ equiv of $\mathbf{3 a}$ was used. DCE $=1,2$-Dichloroethane. 
At first, the reaction conditions were screened with different phosphines using 1a and $\mathrm{PhCOCl}$ (3a) as model substrates (Table S1, entries 2-9). We found that $\mathrm{PPh}_{2} \mathrm{Et}$ has good reactivity than $\mathrm{PPh}_{3}$ whereas nucleophilic $\mathrm{PBu}_{3}$ provided only $7 \%$ yield of the product $4 \mathbf{a a}$. Initial evaluation of bases and solvents with $\mathrm{PPh}_{2}$ Et shown good results (Table S1), but we further optimized with $\mathrm{PPh}_{3}$ because of its significant advantages such as inexpensive, air stable, crystalline, and useful to industrial scale reactions (Table S2). While screening of various solvents, toluene, THF, and EtOAc also showed lower reactivity towards the formation of 4aa accompanied by the formation of the zwitterion 2a (Table S2 entries 2-4). Delightfully, 1,2-dichloroethane (DCE) was found to be the best solvent affording the desired product 4 aa in $76 \%$ yield within $3 \mathrm{~h}$ (Table S2, entry 5). Further improvement in the yield of 4 aa was found by using DIPEA as a base when different bases screened under the reaction conditions (Table S2 entries 6-9). To find the optimal conditions, other factors such as temperature, equivalents of substrates, and addition sequence were further investigated, but no further improvement was observed in the yield of $4 \mathbf{a a}$ (Table S2, entries 10-16). 


\section{Control Experiments:}

Several control experiments have been examined to find out the intermediates by monitoring the ${ }^{31} \mathrm{P}$ NMR analysis (Figure S1). The zwitterion $\mathbf{2 a}(23.7 \mathrm{ppm})$ could be easily prepared in quantitative yields by the reaction of $1 \mathrm{a}$ with $\mathrm{PPh}_{3}$ in $\mathrm{DCE}$ at $30^{\circ} \mathrm{C}$ within $5 \mathrm{~min}$. We further attempted a reaction of the zwitterion 2a with acyl chloride $\mathbf{3 a}$ in the absence of a base. After $6 \mathrm{~h}$, peak at $21.4 \mathrm{ppm}$ was found by diminishing the peak at $23.7 \mathrm{ppm}$ in the reaction mixture by monitoring the ${ }^{31} \mathrm{P}$ NMR, and the phosphonium salt 10aa was further conformed by the ESIHRMS analysis. Notably, the mono-acylated phosphonium species were not found even usage of 1.1 equiv of $\mathrm{PhCOCl}(\mathbf{3 a})$ in the reaction mixture. It could be understood that the acyl transfer is highly feasible and the ylide $\mathbf{C}$ could easily formed even absence of a base under our reaction conditions.

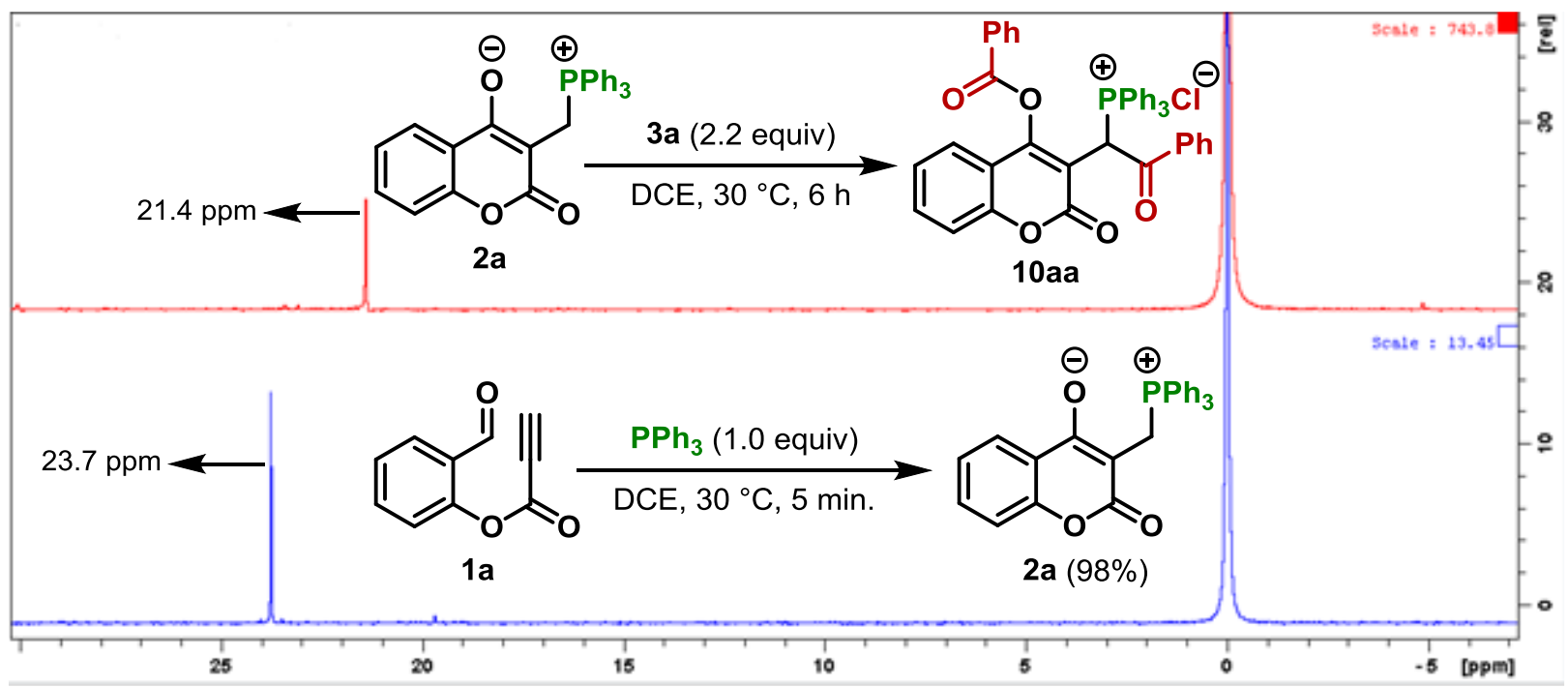

Figure S1. ${ }^{31} \mathrm{P}$ NMR analysis of phosphonium intermediates $\mathbf{2 a}$ and 10aa. $\left(\mathrm{H}_{3} \mathrm{PO}_{4}\right.$ as an internal standard).

(1-(4-(Benzoyloxy)-2-oxo-2H-chromen-3-yl)-2-oxo-2-phenylethyl)triphenylphosphonium chloride (10aa):

${ }^{31} \mathbf{P}$ NMR (162 $\left.\mathrm{MHz} \mathrm{CDCl}_{3}, 25^{\circ} \mathrm{C}\right) \delta / \mathrm{ppm}: 21.4$.

HRMS (ESI) $m / z$ : $[\mathrm{M}]^{+}$Calcd. for $\mathrm{C}_{42} \mathrm{H}_{30} \mathrm{O}_{5} \mathrm{P}$ 645.1831; found 645.1828. 


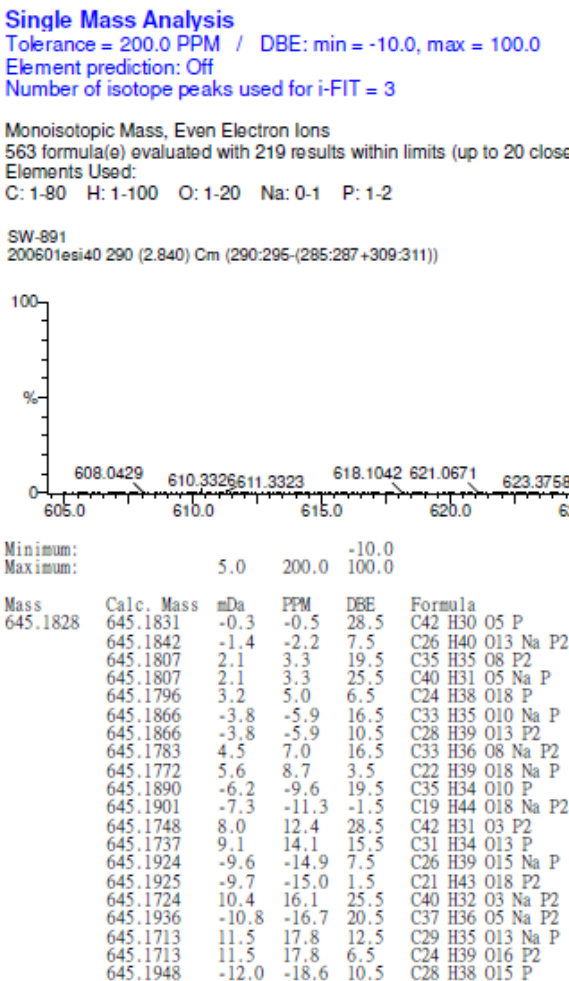

Figure S2. ESI-HRMS analysis of bis-acylated phosphonium salt intermediate 10aa.

Next, we have carried out the reactions using two different acyl chlorides (3a, and 3c) by changing the addition sequence of acyl chlorides under the standard conditions. Interestingly, we could not found any crossover products and only two products 4aa and 4ac were obtained as major and minor products in both the reactions, depending on the first addition of acyl chloride (Scheme S1a). It clearly indicates that the intermolecular $C$-acylation has been ruled out under our conditions, and the rearrangement of betaine intermediate $\mathbf{D}$ would facilitate the intramolecular acyl transfer reaction (manuscript Scheme 6). Furthermore, the phosphorus ylide 11an was found in 53\% yield while 1a mixing with pentafluorobenzoyl chloride (3n) under optimal conditions in $10 \mathrm{~h}$ (Scheme S1b). It was found that the further cyclization and Wittig reaction could not be progressed, presumably, the ortho-substituted fluoro groups hampered the attack of ylide to ester as well as the ylide nucleophilic ability was diminished by the adjacent acyl functionality which was fully substituted with electron-withdrawing fluoro groups. The phosphorus ylide 11an could further characterized by the NMR, ESI-HRMS and X-ray diffraction analysis. 


\section{Scheme S1: Control Experiments: a) Examination of Reaction by Using Two Different Acyl}

\section{Chlorides b) Preparation of bis-Acylated Ylide 11an}

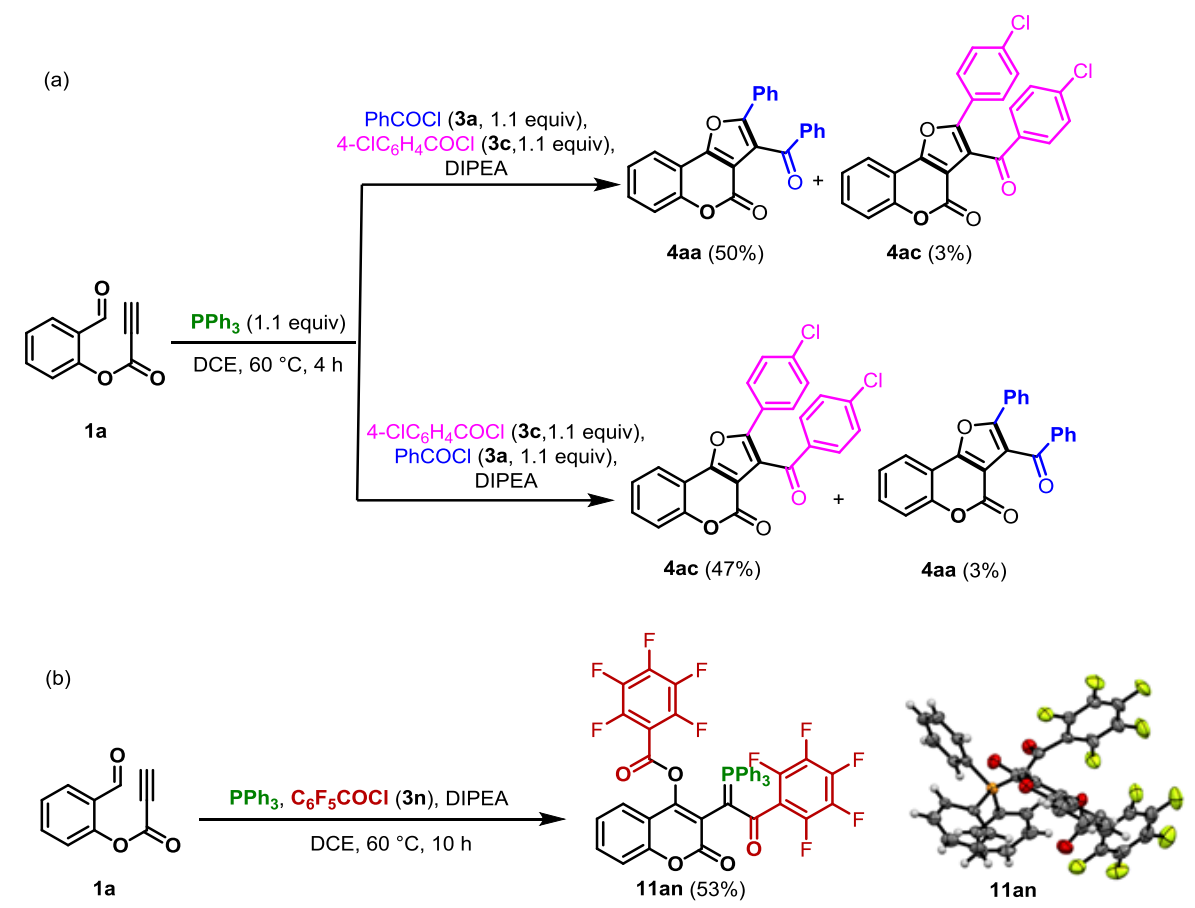

Elemental Composition Report

Page'1

Single Mass Analysis

Tolerance $=200.0$ PPM / DBE: $\min =-10.0, \max =100.0$

Element prediction: Off

Number of isotope peaks used for $\mathrm{i}-\mathrm{FIT}=3$

Monoisotopic Mass, Even Electron lons

11190 formula(e) evaluated with 5223 results within limits (up to 20 closest results for each mass)

$\begin{array}{lllll}\text { C: } 1-80 & \text { H: } 1-100 & \text { O: } 1-15 \quad F: 1-10 & \text { Na: } 0-1 & P: 1-5\end{array}$

David 167
200709esi19 332 (3.239) $\mathrm{Cm}$ (332:338-(313:315+364:366))

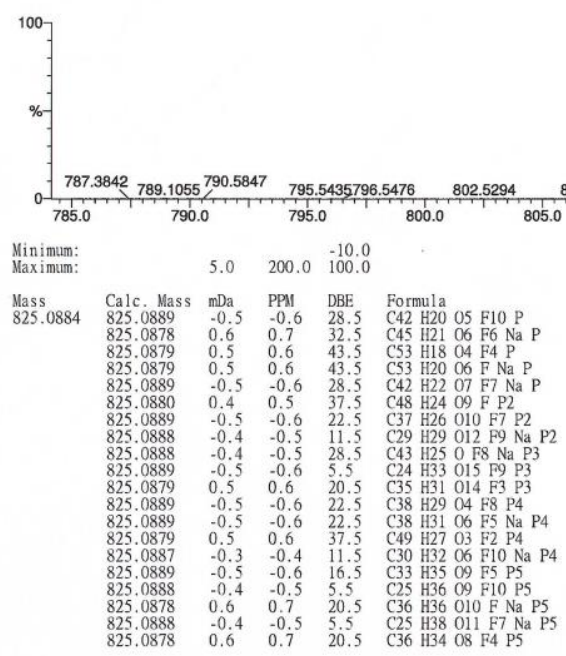

Figure S3. ESI-HRMS analysis of bis-acylated phosphonium ylide intermediate 11an. 


\section{Experimental Procedures:}

a. Typical procedure (TP-1) for the preparation of alkynoates $1 / 9^{1}$ :<smiles>[R]C#CC(=O)O</smiles>

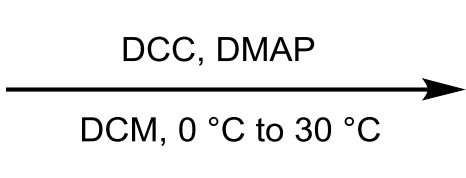

$\mathrm{R}=\mathrm{H}$ or $\mathrm{Ph}$<smiles>[R]C#CC(=O)Oc1ccccc1C=O</smiles>

$1 / 9$

To a mixture of salicylaldehyde (1.04 mL, $10 \mathrm{mmol}, 1.0$ equiv), prop-2-ynoic acid derivative (840 mg, 1.1 equiv), and DMAP (36.7 mg, 0.03 equiv) in anhydrous $\mathrm{CH}_{2} \mathrm{Cl}_{2}(10 \mathrm{~mL})$ cooled to 0 ${ }^{\circ} \mathrm{C}$, and a solution of DCC (2.06 g, 1.0 equiv) in $\mathrm{CH}_{2} \mathrm{Cl}_{2}(10 \mathrm{~mL})$ was added dropwise in $20 \mathrm{~min}$. The reaction mixture was then stirred for 10 minutes at $0{ }^{\circ} \mathrm{C}$ and allowed to $30{ }^{\circ} \mathrm{C}$ for $1-5 \mathrm{~h}$ (monitored by TLC). Precipitated urea was then filtered off and the filtrate was evaporated in vacuum. The crude residue was subjected to flash column chromatography on silica gel (hexanes/EA: 9:1) to obtain the pure alkynoate 1/9.

b. Typical procedure (TP-2) for the preparation of functionalized furo[3,2-c]coumarins 4:<smiles>[R][M]1ccc(OC(=O)C#C)c(C=O)c1</smiles>

1

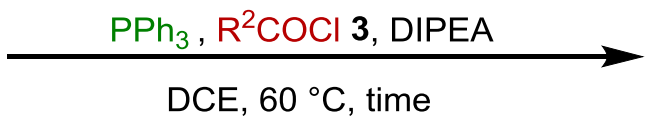

DCE, $60^{\circ} \mathrm{C}$, time<smiles>[R]C(=O)c1c([R])oc2c3c(oc(=O)c12)C=C[R1]C=C3</smiles>

4

A dry and argon-flushed $10 \mathrm{~mL}$ round bottom flask equipped with a magnetic stir bar was sequentially charged with 1 ( $0.3 \mathrm{mmol}), \mathrm{PPh}_{3}$ (1.1 equiv), anhydrous DCE ( $\left.3 \mathrm{~mL}\right)$, acyl chloride 3 (2.2 equiv) and DIPEA (1.5 equiv) at $30^{\circ} \mathrm{C}$. The reaction mixture was stirred at $60^{\circ} \mathrm{C}$ for $2-12$ hours in an oil bath. After completion of the reaction, as observed by ${ }^{1} \mathrm{H}$ NMR analysis, solvent was removed in vacuo and the crude residue was subjected to flash column chromatography on silica gel to obtain the product 4 .

c. Typical procedure (TP-3) for the preparation of 2,3-disubstituted furo[3,2-c]coumarins 6: 
<smiles>O=Cc1ccccc1OC(=O)C#Cc1ccccc1</smiles>

9

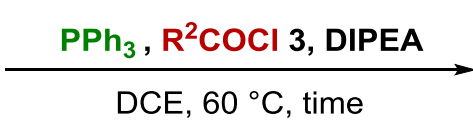

DCE, $60^{\circ} \mathrm{C}$, time<smiles>[R]c1cc2c(=O)oc3c(c2o1)C=C[R1]C=C3</smiles>

6

A dry and argon-flushed $10 \mathrm{~mL}$ round bottom flask equipped with a magnetic stir bar was sequentially charged with 9 (0.3 mmol), $\mathrm{PPh}_{3}$ (1.1 equiv), anhydrous DCE ( $\left.3 \mathrm{~mL}\right)$, acyl chloride 3 (1.1 equiv) and DIPEA ( 1.5 equiv) at $30{ }^{\circ} \mathrm{C}$. The reaction mixture was stirred at $60{ }^{\circ} \mathrm{C}$ for $2-12$ hours in an oil bath. After completion of the reaction, as observed by ${ }^{1} \mathrm{H}$ NMR analysis, solvent was removed in vacuo and the crude residue was subjected to flash column chromatography on silica gel to obtain the product $\mathbf{6}$, and all the desired products $\mathbf{6}$ are intense-fluorescent under UV light.

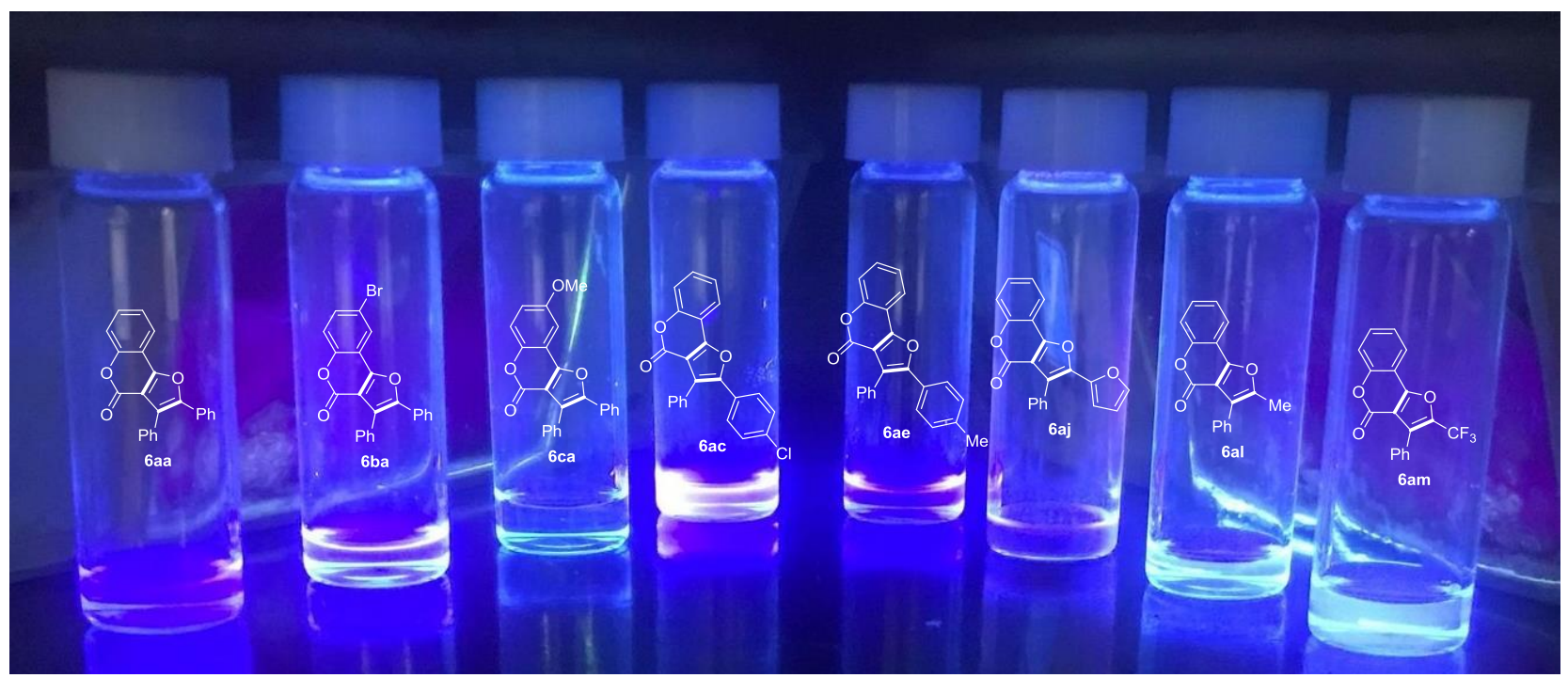

Figure S4. Selected compounds of furo[3,2-c] coumarin 6 solution in $\mathrm{CH}_{2} \mathrm{Cl}_{2}$ are intensely fluorescence under UV light.

\section{d. Typical procedure (TP-4) for the preparation of furo[3,2-c]quinolinones 7:}


<smiles>O=Cc1ccccc1NC(=O)C#Cc1ccccc1</smiles>

$12 a$

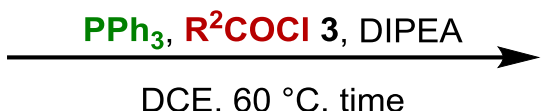

DCE, $60^{\circ} \mathrm{C}$, time

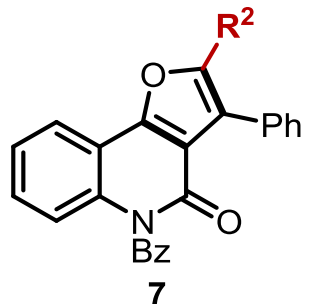

7

A dry and argon-flushed $10 \mathrm{~mL}$ round bottom flask equipped with a magnetic stir bar was sequentially charged with 12a $(0.3 \mathrm{mmol}), \mathrm{PPh}_{3}$ (1.1 equiv), anhydrous DCE ( $\left.3 \mathrm{~mL}\right)$, acyl chloride 3 (1.1 equiv) and DIPEA (1.5 equiv) at $30{ }^{\circ} \mathrm{C}$. The reaction mixture was stirred at $60{ }^{\circ} \mathrm{C}$ for $3-4$ hours in an oil bath. After completion of the reaction, as observed by ${ }^{1} \mathrm{H}$ NMR analysis, solvent was removed in vacuo and the crude residue was subjected to flash column chromatography on silica gel to obtain the product 7 .

\section{Analytical Data for All New Compounds:}

\section{2-Formylphenyl propiolate (1a)}<smiles>C#CC(=O)Oc1ccccc1C=O</smiles>

Following the TP-1, 1a was obtained from Salicylaldehyde (1044 $\mu \mathrm{L}, 10 \mathrm{mmol})$, prop-2-ynoic acid, (680 $\mu \mathrm{L}, 1.1$ equiv.), DMAP (36.7 mg, 0.03 equiv.), and DCC (2063 mg, 1.0 equiv.) at 0 to $30{ }^{\circ} \mathrm{C}$. The residue was purified by column chromatography $\left(\mathrm{SiO}_{2}, \mathrm{Hexanes} / \mathrm{EtOAc}=90: 10\right)$ to give as a white solid $(957.7 \mathrm{mg}, 55 \%$ yield $) ; \mathrm{R}_{f}=0.31(\mathrm{EA}: \mathrm{Hex}=1: 6) ; \mathrm{mp}=49.3-50.1^{\circ} \mathrm{C}$.

${ }^{1} \mathbf{H}$ NMR (400 MHz, $\left.\mathrm{CDCl}_{3}, 25^{\circ} \mathrm{C}\right) \delta / \mathrm{ppm}: 10.14(\mathrm{~s}, 1 \mathrm{H}), 7.90(\mathrm{dd}, J=7.5,1.7 \mathrm{~Hz}, 1 \mathrm{H}), 7.66(\mathrm{td}$, $J=7.5,1.7 \mathrm{~Hz}, 1 \mathrm{H}), 7.45(\mathrm{t}, J=7.5 \mathrm{~Hz}, 1 \mathrm{H}), 7.25(\mathrm{~d}, J=8.0 \mathrm{~Hz}, 1 \mathrm{H}), 3.22(\mathrm{~s}, 1 \mathrm{H})$.

${ }^{13} \mathrm{C}$ NMR $\left(100 \mathrm{MHz} \mathrm{CDCl}_{3}, 25^{\circ} \mathrm{C}\right) \delta / \mathrm{ppm}: 188.1,150.4,150.0,135.4,131.2,127.6,127.2,123.0$, 77.9, 73.6 .

IR $(\mathrm{KBr}) \tilde{v}\left(\mathrm{~cm}^{-1}\right):$ 3260, 2361, 2126, 1737, 1703, 1606, 1188, 914, 758.

HRMS (FAB) $m / z:[\mathrm{M}+\mathrm{H}]^{+}$Calcd. for $\mathrm{C}_{10} \mathrm{H}_{7} \mathrm{O}_{3}$ 175.0395; found 175.0392.

\section{4-Bromo-2-formylphenyl propiolate (1b)}




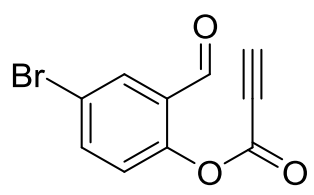

Following the TP-1, 1b was obtained from 5-bromo-2-hydroxybenzaldehyde (2010 mg, $10 \mathrm{mmol}$ ), prop-2-ynoic acid, (680 $\mu \mathrm{L}, 1.1$ equiv.), DMAP (36.7 mg, 0.03 equiv.), and DCC (2063 mg, 1.0 equiv.) at 0 to $30^{\circ} \mathrm{C}$. The residue was purified by column chromatography $\left(\mathrm{SiO}_{2}, \mathrm{Hexanes} / \mathrm{EtOAc}=\right.$ 91:9) to give as a white solid (1062 $\mathrm{mg}, 42 \%$ yield); $\mathrm{R}_{f}=0.36(\mathrm{EA}: \mathrm{Hex}=1: 6) ; \mathrm{mp}=103.0-103.7$ ${ }^{\circ} \mathrm{C}$.

${ }^{1} \mathbf{H}$ NMR $\left(400 \mathrm{MHz}, \mathrm{CDCl}_{3}, 25^{\circ} \mathrm{C}\right) \delta / \mathrm{ppm}: 10.09$ (s, 1H), $8.03(\mathrm{~d}, J=2.4 \mathrm{~Hz}, 1 \mathrm{H}), 7.76(\mathrm{dd}, J=$ 8.7, $2.4 \mathrm{~Hz}, 1 \mathrm{H}), 7.17$ (d, $J=8.8 \mathrm{~Hz}, 1 \mathrm{H}), 3.20(\mathrm{~s}, 1 \mathrm{H})$.

${ }^{13} \mathrm{C} \mathrm{NMR}\left(100 \mathrm{MHz}, \mathrm{CDCl}_{3}, 25^{\circ} \mathrm{C}\right) \delta / \mathrm{ppm}: 186.6,150.0,149.3,138.1,133.4,128.9,124.9,120.6$, 78.3, 73.4 .

IR (KBr) $\tilde{v}\left(\mathrm{~cm}^{-1}\right): 3266,2362,2127,1736,1707,1472,1185,887,740$.

HRMS (FAB) $m / z:[\mathrm{M}+\mathrm{H}]^{+}$Calcd. for $\mathrm{C}_{10} \mathrm{H}_{6}{ }^{79} \mathrm{BrO}_{3} 252.9500$; found 252.9494.

HRMS (FAB) $m / z:[\mathrm{M}+\mathrm{H}]^{+}$Calcd. for $\mathrm{C}_{10} \mathrm{H}_{6}{ }^{81} \mathrm{BrO}_{3} 254.9480$; found 254.9499.

\section{4-Chloro-2-formylphenyl propiolate (1c)}

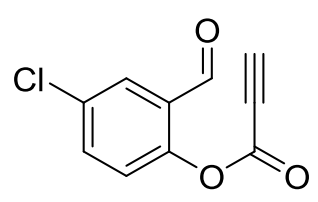

Following the TP-1, 1c was obtained from 5-chloro-2-hydroxybenzaldehyde (1565 mg, $10 \mathrm{mmol})$, prop-2-ynoic acid, (680 $\mu \mathrm{L}, 1.1$ equiv.), DMAP (36.7 mg, 0.03 equiv.), and DCC (2063 mg, 1.0 equiv.) at 0 to $30^{\circ} \mathrm{C}$. The residue was purified by column chromatography $\left(\mathrm{SiO}_{2}, \mathrm{Hexanes} / \mathrm{EtOAc}=\right.$ 91:9) to give as a white solid (813.9 $\mathrm{mg}, 39 \%$ yield); $\mathrm{R}_{f}=0.36(\mathrm{EA}: \mathrm{Hex}=1: 6) ; \mathrm{mp}=83.5-84.3{ }^{\circ} \mathrm{C}$. ${ }^{1} \mathbf{H}$ NMR (400 MHz, $\left.\mathrm{CDCl}_{3}, 25^{\circ} \mathrm{C}\right) \delta / \mathrm{ppm}: 10.10(\mathrm{~s}, 1 \mathrm{H}), 7.88(\mathrm{~d}, J=2.7 \mathrm{~Hz}, 1 \mathrm{H}), 7.61(\mathrm{dd}, J=$ 8.8, $2.9 \mathrm{~Hz}, 1 \mathrm{H}), 7.24$ (d, $J=8.8 \mathrm{~Hz}, 1 \mathrm{H}), 3.21(\mathrm{~s}, 1 \mathrm{H})$.

${ }^{13} \mathrm{C}$ NMR $\left(100 \mathrm{MHz} \mathrm{CDCl}_{3}, 25^{\circ} \mathrm{C}\right) \delta / \mathrm{ppm}: 186.6,150.1,148.8,135.1,133.2,130.3,128.7,124.6$, $78.3,73.4$.

IR $(\mathrm{KBr}) \tilde{v}\left(\mathrm{~cm}^{-1}\right): 3261,2362,2125,1734,1693,1474,1175,895,732$.

HRMS (FAB) $m / z:[\mathrm{M}+\mathrm{H}]^{+}$Calcd. for $\mathrm{C}_{10} \mathrm{H}_{6}{ }^{35} \mathrm{ClO}_{3} 209.0005$; found 209.0008. 
HRMS (FAB) $m / z:[\mathrm{M}+\mathrm{H}]^{+}$Calcd. for $\mathrm{C}_{10} \mathrm{H}_{6}{ }^{37} \mathrm{ClO}_{3} 210.9976$; found 211.0001 .

\section{4-Fluoro-2-formylphenyl propiolate (1d)}

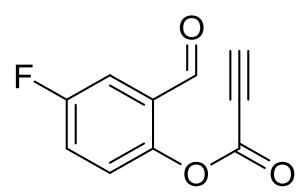

Following the TP-1, 1d was obtained from 5-fluoro-2-hydroxybenzaldehyde (1401 mg, $10 \mathrm{mmol}$ ), prop-2-ynoic acid, (680 $\mu \mathrm{L}, 1.1$ equiv.), DMAP (36.7 mg, 0.03 equiv.), and DCC (2063 mg, 1.0 equiv.) at 0 to $30^{\circ} \mathrm{C}$. The residue was purified by column chromatography $\left(\mathrm{SiO}_{2}, \mathrm{Hexanes} / \mathrm{EtOAc}=\right.$ 90:10) to give as a white solid (806.8 $\mathrm{mg}, 42 \%$ yield); $\mathrm{R}_{f}=0.32$ (EA:Hex =1:6); $\mathrm{mp}=75.7-76.6$ ${ }^{\circ} \mathrm{C}$.

${ }^{1} \mathbf{H}$ NMR $\left(400 \mathrm{MHz} \mathrm{CDCl}_{3}, 25^{\circ} \mathrm{C}\right) \delta / \mathrm{ppm}: 10.11(\mathrm{~s}, 1 \mathrm{H}), 7.60(\mathrm{dd}, J=8.1,3.1 \mathrm{~Hz}, 1 \mathrm{H}), 7.36$ (dddd, $J=7.3,3.1 \mathrm{~Hz}, 1 \mathrm{H}), 7.26(\mathrm{dd}, J=8.7,4.4 \mathrm{~Hz}, 1 \mathrm{H}), 3.20(\mathrm{~s}, 1 \mathrm{H})$.

${ }^{13} \mathrm{C}$ NMR $\left(100 \mathrm{MHz}, \mathrm{CDCl}_{3}, 25{ }^{\circ} \mathrm{C}\right) \delta / \mathrm{ppm}: 186.6,160.6\left(\mathrm{~d},{ }^{1} J_{\mathrm{C}-\mathrm{F}}=250 \mathrm{~Hz}\right), 150.4,146.4\left(\mathrm{~d},{ }^{4} J_{\mathrm{C}-}\right.$ $\mathrm{F}=2.9 \mathrm{~Hz}), 129.0\left(\mathrm{~d},{ }^{3} J_{\mathrm{C}-\mathrm{F}}=6.3 \mathrm{~Hz}\right), 124.90\left(\mathrm{~d},{ }^{3} J_{\mathrm{C}-\mathrm{F}}=8.0 \mathrm{~Hz}\right), 122.3\left(\mathrm{~d},{ }^{2} J_{\mathrm{C}-\mathrm{F}}=24.0 \mathrm{~Hz}\right), 116.4$ $\left(\mathrm{d},{ }^{2} J_{\mathrm{C}-\mathrm{F}}=24.3 \mathrm{~Hz}\right), 78.2,73.4$.

${ }^{19}$ F NMR $\left(376 \mathrm{MHz}, \mathrm{CDCl}_{3}, 25^{\circ} \mathrm{C}\right) \delta / \mathrm{ppm}:-113.05$.

IR (KBr) $\tilde{v}\left(\mathrm{~cm}^{-1}\right): 3269,2363,2127,1735,1701,1487,1264,1188,909,743$.

HRMS (FAB) $m / z:[\mathrm{M}+\mathrm{H}]^{+}$Calcd. for $\mathrm{C}_{10} \mathrm{H}_{6} \mathrm{FO}_{3}$ 193.0301; found 193.0302.

\section{2-Formyl-4-methylphenyl propiolate (1e)}

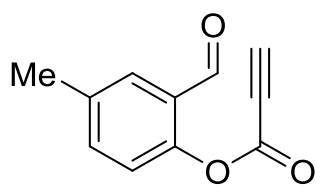

Following the TP-1, 1e was obtained from 2-hydroxy-5-methylbenzaldehyde (1361 mg, $10 \mathrm{mmol}$ ), prop-2-ynoic acid, (680 $\mu \mathrm{L}, 1.1$ equiv.), DMAP (36.7 mg, 0.03 equiv.), and DCC (2063 mg, 1.0 equiv.) at 0 to $30^{\circ} \mathrm{C}$. The residue was purified by column chromatography $\left(\mathrm{SiO}_{2}, \mathrm{Hexanes} / \mathrm{EtOAc}=\right.$ 91:9) to give as a white solid (658.4 mg, 35\% yield); $\mathrm{R}_{f}=0.39$ (EA:Hex $\left.=1: 6\right) ; \mathrm{mp}=64.8-65.7{ }^{\circ} \mathrm{C}$. ${ }^{1} \mathbf{H}$ NMR (400 MHz, $\left.\mathrm{CDCl}_{3}, 25{ }^{\circ} \mathrm{C}\right) \delta / \mathrm{ppm}: 10.1(\mathrm{~s}, 1 \mathrm{H}), 7.69(\mathrm{~d}, J=1.9 \mathrm{~Hz}, 1 \mathrm{H}), 7.45(\mathrm{dd}, J=$ 8.5, $2.5 \mathrm{~Hz}, 1 \mathrm{H}), 7.13(\mathrm{~d}, J=8.4 \mathrm{~Hz}, 1 \mathrm{H}), 3.18(\mathrm{~s}, 1 \mathrm{H}), 2.42(\mathrm{~s}, 3 \mathrm{H})$. 
${ }^{13} \mathrm{C} \mathrm{NMR}\left(100 \mathrm{MHz}, \mathrm{CDCl}_{3}, 25^{\circ} \mathrm{C}\right) \delta / \mathrm{ppm}: 188.2,150.6,148.1,137.3,136.0,131.3,127.3,122.7$, 77.6, 73.7, 20.6 .

IR $(\mathrm{KBr}) \tilde{v}\left(\mathrm{~cm}^{-1}\right): 3247,2362,2126,1729,1700,1493,1203,891,753$.

HRMS (FAB) $m / z:[\mathrm{M}+\mathrm{H}]^{+}$Calcd. for $\mathrm{C}_{11} \mathrm{H}_{9} \mathrm{O}_{3}$ 189.0552; found.189.0553

2-Formyl-4-methoxyphenyl propiolate (1f)

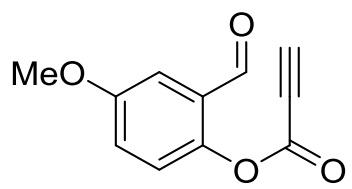

Following the TP-1, 1 f was obtained from 2-hydroxy-5-methoxybenzaldehyde (1247 $\mu \mathrm{L}, 10$ mmol), prop-2-ynoic acid, (680 $\mu \mathrm{L}, 1.1$ equiv.), DMAP (36.7 mg, 0.03 equiv.), and DCC (2063 mg, 1.0 equiv.) at 0 to $30{ }^{\circ} \mathrm{C}$. The residue was purified by column chromatography $\left(\mathrm{SiO}_{2}\right.$, Hexanes/EtOAc= 90:10) to give as a white solid $(755.2 \mathrm{mg}, 37 \%$ yield $) ; \mathrm{R}_{f}=0.24(\mathrm{EA}: \mathrm{Hex}=1: 6)$; $\mathrm{mp}=84.4-85.3{ }^{\circ} \mathrm{C}$.

${ }^{1} \mathbf{H}$ NMR (400 MHz, $\left.\mathrm{CDCl}_{3}, 25^{\circ} \mathrm{C}\right) \delta / \mathrm{ppm}: 10.12(\mathrm{~s}, 1 \mathrm{H}), 7.37$ (t, $\left.J=1.8 \mathrm{~Hz}, 1 \mathrm{H}\right), 7.17(\mathrm{~d}, J=1.8$ $\mathrm{Hz}, 2 \mathrm{H}), 3.86$ (s, 3H), 3.17 (s, 1H).

${ }^{13} \mathrm{C}$ NMR $\left(100 \mathrm{MHz}, \mathrm{CDCl}_{3}, 25{ }^{\circ} \mathrm{C}\right) \delta / \mathrm{ppm}: 187.7,158.1,150.9,144.3,128.2,124.1,122.0,113.1$, 77.8, 73.7, 55.9.

IR (KBr) $\tilde{v}\left(\mathrm{~cm}^{-1}\right):$ 3259, 2356, 2125, 1732, 1696, 1492, 1276, 1196, 1033, 741.

HRMS (FAB) $m / z:[\mathrm{M}+\mathrm{H}]^{+}$Calcd. for $\mathrm{C}_{11} \mathrm{H}_{9} \mathrm{O}_{4} 205.0501$; found 205.0502 .

\section{2-Formyl-5-methoxyphenyl propiolate (1g)}

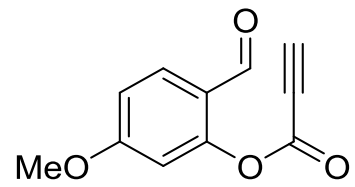

Following the TP-1, $1 \mathrm{~g}$ was obtained from 2-hydroxy-4-methoxybenzaldehyde (1521 mg, 10 mmol), prop-2-ynoic acid, (680 $\mu \mathrm{L}, 1.1$ equiv.), DMAP (36.7 mg, 0.03 equiv.), and DCC (2063 mg, 1.0 equiv.) at 0 to $30{ }^{\circ} \mathrm{C}$. The residue was purified by column chromatography $\left(\mathrm{SiO}_{2}\right.$, Hexanes/EtOAc $=88: 12)$ to give as a white solid $(796.0 \mathrm{mg}, 39 \%$ yield $) ; \mathrm{R}_{f}=0.18(\mathrm{EA}: \mathrm{Hex}=1: 6)$; $\mathrm{mp}=88.1-89.0{ }^{\circ} \mathrm{C}$.

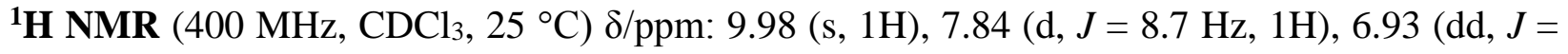
8.8, $2.6 \mathrm{~Hz}, 1 \mathrm{H}), 6.73$ (d, $J=2.2 \mathrm{~Hz}, 1 \mathrm{H}), 3.88$ (s, 3H), $3.16(\mathrm{~s}, 1 \mathrm{H})$. 
${ }^{13} \mathbf{C} \mathbf{N M R}\left(100 \mathrm{MHz}, \mathrm{CDCl}_{3}, 25{ }^{\circ} \mathrm{C}\right) \delta / \mathrm{ppm}: 186.9,165.2,151.9,150.3,133.1,121.2,112.9,108.5$, 77.6, 73.7, 56.0.

IR (KBr) $\tilde{v}\left(\mathrm{~cm}^{-1}\right): 3255,2350,2123,1734,1693,1609,1252,1184,904,740$.

HRMS (FAB) $m / z:[\mathrm{M}+\mathrm{H}]^{+}$Calcd. for $\mathrm{C}_{11} \mathrm{H}_{9} \mathrm{O}_{4}$ 205.0501; found 205.0504 .

\section{2-Formyl-6-methoxyphenyl propiolate (1h)}

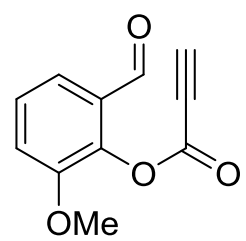

Following the TP-1, $\mathbf{1 h}$ was obtained from 2-hydroxy-3-methoxybenzaldehyde (1521 mg, 10 mmol), prop-2-ynoic acid, (680 $\mu \mathrm{L}, 1.1$ equiv.), DMAP (36.7 mg, 0.03 equiv.), and DCC (2063 mg, 1.0 equiv.) at 0 to $30{ }^{\circ} \mathrm{C}$. The residue was purified by column chromatography $\left(\mathrm{SiO}_{2}\right.$, Hexanes/EtOAc $=88: 12$ ) to give as a white solid (1020 mg, 50\% yield); $\mathrm{R}_{f}=0.19$ (EA:Hex $=1: 6$ ); $\mathrm{mp}=76.1-77.0^{\circ} \mathrm{C}$.

${ }^{1} \mathrm{H}$ NMR $\left(400 \mathrm{MHz}, \mathrm{CDCl}_{3}, 25{ }^{\circ} \mathrm{C}\right) \delta / \mathrm{ppm}: 10.17(\mathrm{~s}, 1 \mathrm{H}), 7.48(\mathrm{dd}, J=7.7,1.4 \mathrm{~Hz}, 1 \mathrm{H}), 7.38(\mathrm{t}$, $J=8.1 \mathrm{~Hz}, 1 \mathrm{H}), 7.24(\mathrm{dd}, J=8.0,1.7 \mathrm{~Hz}, 1 \mathrm{H}), 3.89$ (s, 3H), 3.14 (s, 1H).

${ }^{13} \mathrm{C}$ NMR $\left(100 \mathrm{MHz}, \mathrm{CDCl}_{3}, 25{ }^{\circ} \mathrm{C}\right) \delta / \mathrm{ppm}: 188.1,151.4,149.8,140.1,128.9,127.6,121.1,118.0$, 77.5, 73.6, 56.4.

IR $(\mathrm{KBr}) \tilde{v}\left(\mathrm{~cm}^{-1}\right): 3260,2362,2126,1736,1702,1481,1280,1186,951,783$.

HRMS (FAB) $m / z:[\mathrm{M}+\mathrm{H}]^{+}$Calcd. for $\mathrm{C}_{11} \mathrm{H}_{9} \mathrm{O}_{4}$ 205.0501; found 205.0499.

\section{1-Formylnaphthalen-2-yl propiolate (1i)}

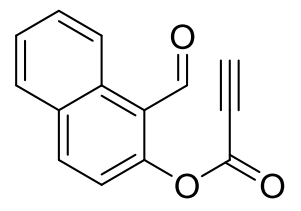

Following the TP-1, 1i was obtained from 2-hydroxy-1-naphthaldehyde (1721 mg, $10 \mathrm{mmol}$ ), prop-2-ynoic acid, (680 $\mu \mathrm{L}, 1.1$ equiv.), DMAP (36.7 mg, 0.03 equiv.), and DCC (2063 mg, 1.0 equiv.) at 0 to $30^{\circ} \mathrm{C}$. The residue was purified by column chromatography $\left(\mathrm{SiO}_{2}, \mathrm{Hexanes} / \mathrm{EtOAc}=\right.$ 90:10) to give as a white solid (964.1 mg, $43 \%$ yield); $\mathrm{R}_{f}=0.35$ (EA:Hex $\left.=1: 6\right) ; \mathrm{mp}=99.5-100.3$ ${ }^{\circ} \mathrm{C}$. 
${ }^{1} \mathrm{H}$ NMR (400 MHz, $\left.\mathrm{CDCl}_{3}, 25^{\circ} \mathrm{C}\right)$ d/ppm: $10.74(\mathrm{~s}, 1 \mathrm{H}), 9.13(\mathrm{~d}, J=8.6 \mathrm{~Hz}, 1 \mathrm{H}), 8.12(\mathrm{~d}, J=$ $8.9 \mathrm{~Hz}, 1 \mathrm{H}), 7.89(\mathrm{~d}, J=7.9 \mathrm{~Hz}, 1 \mathrm{H}), 7.70(\mathrm{td}, J=7.9,1.6 \mathrm{~Hz}, 1 \mathrm{H}), 7.58(\mathrm{td}, J=7.8,1.5 \mathrm{~Hz}, 1 \mathrm{H})$, $7.32(\mathrm{~d}, J=8.9 \mathrm{~Hz}, 1 \mathrm{H}), 3.20(\mathrm{~s}, 1 \mathrm{H})$.

${ }^{13} \mathrm{C} \mathrm{NMR}\left(100 \mathrm{MHz}, \mathrm{CDCl}_{3}, 25{ }^{\circ} \mathrm{C}\right) \delta / \mathrm{ppm}: 189.5,152.9,150.5,136.6,132.1,131.0,129.8,128.5$, 127.0, 125.2, 121.2, 120.8, 78.2, 73.5.

IR $(\mathrm{KBr}) \tilde{v}\left(\mathrm{~cm}^{-1}\right): 3251,2362,2122,1733,1681,1513,1189,1064,772$.

HRMS (FAB) $m / z:[\mathrm{M}+\mathrm{H}]^{+}$Calcd. for $\mathrm{C}_{14} \mathrm{H}_{9} \mathrm{O}_{3}$ 225.0552; found 225.0553 .

\section{2-Formylphenyl 3-phenylpropiolate (9a)}

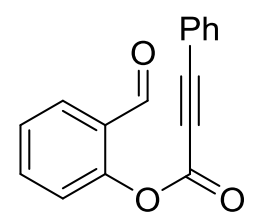

Following the TP-1, 9a was obtained from Salicylaldehyde (522 $\mu \mathrm{L}, 5 \mathrm{mmol})$, Phenylpropiolic acid, (803.8 mg, 1.1 equiv.), DMAP (18.3 mg, 0.03 equiv.), and DCC (1031 mg, 1.0 equiv.) at 0 to $30{ }^{\circ} \mathrm{C}$. The residue was purified by column chromatography $\left(\mathrm{SiO}_{2}\right.$, Hexanes/EtOAc $\left.=90: 10\right)$ to give as a white solid (738.2 mg, 59\% yield); $\mathrm{R}_{f}=0.34$ (EA:Hex $\left.=1: 6\right) ; \mathrm{mp}=80.3-81.2{ }^{\circ} \mathrm{C}$.

${ }^{1} \mathbf{H}$ NMR $\left(400 \mathrm{MHz}, \mathrm{CDCl}_{3}, 25{ }^{\circ} \mathrm{C}\right) \delta / \mathrm{ppm}: 10.23(\mathrm{~s}, 1 \mathrm{H}), 7.94(\mathrm{dd}, J=7.9,1.8 \mathrm{~Hz}, 1 \mathrm{H}), 7.68(\mathrm{dd}$, $J=7.8,1.8 \mathrm{~Hz}, 1 \mathrm{H}), 7.66(\mathrm{~d}, J=7.5 \mathrm{~Hz}, 2 \mathrm{H}), 7.50(\mathrm{t}, J=7.6 \mathrm{~Hz}, 1 \mathrm{H}), 7.45(\mathrm{~d}, J=7.6 \mathrm{~Hz}, 1 \mathrm{H})$, $7.42(\mathrm{pt}, J=7.8 \mathrm{~Hz}, 2 \mathrm{H}), 7.30(\mathrm{~d}, J=8.1 \mathrm{~Hz}, 1 \mathrm{H})$.

${ }^{13} \mathrm{C} \mathrm{NMR}\left(100 \mathrm{MHz}, \mathrm{CDCl}_{3}, 25^{\circ} \mathrm{C}\right) \delta / \mathrm{ppm}: 188.2,151.8,151.0,135.4,133.3,131.3,130.5,128.7$, 127.9, 127.0, 123.2, 118.9, 89.8, 79.6.

IR $(\mathrm{KBr}) \tilde{v}\left(\mathrm{~cm}^{-1}\right): 3245,2230,1731,1699,1281,1140,931,756$.

HRMS (ESI) $m / z:[\mathrm{M}+\mathrm{Na}]^{+} \mathrm{Calcd}$. for $\mathrm{C}_{16} \mathrm{H}_{10} \mathrm{O}_{3} \mathrm{Na} 273.0528$; found 273.0528 .

\section{4-Bromo-2-formylphenyl 3-phenylpropiolate (9b)}<smiles>O=Cc1cc(Br)ccc1OC(=O)C#CPc1ccccc1</smiles>

Following the TP-1, 9b was obtained from 5-bromo-2-hydroxybenzaldehyde (1005 mg, $5 \mathrm{mmol}$ ), Phenylpropiolic acid, (803.8 mg, 1.1 equiv.), DMAP (18.3 mg, 0.03 equiv.), and DCC (1031 mg, 1.0 equiv.) at 0 to $30{ }^{\circ} \mathrm{C}$. The residue was purified by column chromatography $\left(\mathrm{SiO}_{2}\right.$, 
Hexanes/EtOAc $=93: 7)$ to give as a white solid $(789.8 \mathrm{mg}, 48 \%$ yield $) ; \mathrm{R}_{f}=0.57(\mathrm{EA}: \mathrm{Hex}=1: 6)$; $\mathrm{mp}=91.9-92.8^{\circ} \mathrm{C}$.

${ }^{1} \mathbf{H}$ NMR $\left(400 \mathrm{MHz} \mathrm{CDCl}_{3}, 25^{\circ} \mathrm{C}\right) \delta / \mathrm{ppm}: 10.18(\mathrm{~s}, 1 \mathrm{H}), 8.05(\mathrm{~d}, J=2.6 \mathrm{~Hz}, 1 \mathrm{H}), 7.77(\mathrm{dd}, J=$ 8.8, $2.5 \mathrm{~Hz}, 1 \mathrm{H}), 7.66(\mathrm{~d}, J=7.9 \mathrm{~Hz}, 2 \mathrm{H}), 7.53(\mathrm{t}, J=7.4 \mathrm{~Hz}, 1 \mathrm{H}), 7.43(\mathrm{t}, J=7.6 \mathrm{~Hz}, 2 \mathrm{H}), 7.22$ $(\mathrm{d}, J=8.3 \mathrm{~Hz}, 1 \mathrm{H})$.

${ }^{13} \mathrm{C}$ NMR $\left(100 \mathrm{MHz}, \mathrm{CDCl}_{3}, 25{ }^{\circ} \mathrm{C}\right) \delta / \mathrm{ppm}: 186.7,151.3,150.0,138.1,133.4,132.8,131.5,129.1$, 128.7, 125.0, 120.4, 118.7, 90.3, 79.3.

IR $(\mathrm{KBr}) \tilde{v}\left(\mathrm{~cm}^{-1}\right): 3069,2231,1731,1709,1471,1282,1138,880,757$.

HRMS (ESI) $m / z$ : [M+Na $]^{+}$Calcd. for $\mathrm{C}_{16} \mathrm{H}_{9}{ }^{79} \mathrm{BrO}_{3} \mathrm{Na} 350.9633$; found 350.9634 .

HRMS (ESI) $m / z$ : [M+Na] ${ }^{+}$Calcd. for $\mathrm{C}_{16} \mathrm{H}_{9}{ }^{81} \mathrm{BrO}_{3} \mathrm{Na} 352.9612$; found 352.9617.

2-Formyl-4-methoxyphenyl 3-phenylpropiolate (9c)

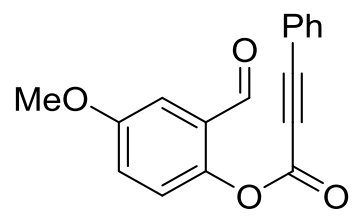

Following the TP-1, 9c was obtained from 2-hydroxy-5-methoxybenzaldehyde $(624.0 \mu \mathrm{L}, 5$ mmol), Phenylpropiolic acid, (682 mg, 1.1 equiv.), DMAP (18.3 mg, 0.03 equiv.), and DCC (1031 mg, 1.0 equiv.) at 0 to $30{ }^{\circ} \mathrm{C}$. The residue was purified by column chromatography $\left(\mathrm{SiO}_{2}\right.$, Hexanes/EtOAc= 90:10) to give as a white solid (602.5 mg, 43\% yield); $\mathrm{R}_{f}=0.38(\mathrm{EA}: \mathrm{Hex}=1: 6)$; $\mathrm{mp}=104.1-105.0^{\circ} \mathrm{C}$.

${ }^{1} \mathbf{H}$ NMR $\left(400 \mathrm{MHz}, \mathrm{CDCl}_{3}, 25^{\circ} \mathrm{C}\right) \delta / \mathrm{ppm}: 10.19(\mathrm{~s}, 1 \mathrm{H}), 7.64(\mathrm{~d}, J=7.7 \mathrm{~Hz}, 2 \mathrm{H}), 7.50(\mathrm{t}, J=7.5$ $\mathrm{Hz}, 1 \mathrm{H}), 7.44-7.38$ (m, 3H), 7.24-7.16 (m, 2H), 3.85 (s, 3H).

${ }^{13} \mathrm{C}$ NMR (100 MHz, $\left.\mathrm{CDCl}_{3}, 25^{\circ} \mathrm{C}\right) \delta / \mathrm{ppm}: 187.8,157.9,152.2,145.0,133.2,131.2,128.7,128.3$, 124.2, 122.1, 118.9, 112.3, 89.7, 79.6, 55.8.

IR $(\mathrm{KBr}) \tilde{v}\left(\mathrm{~cm}^{-1}\right):$ 3070, 2359, 2231, 1729, 1697, 1489, 1275, 1142, 1032, 758.

HRMS (ESI) $m / z$ : [M+Na] $]^{+}$Calcd. for $\mathrm{C}_{17} \mathrm{H}_{12} \mathrm{O}_{4} \mathrm{Na} 303.0633$; found 303.0633.

General procedure for the synthesis of $N$-(2-formylphenyl)- $N$-(3-phenylpropioloyl) benzamide (12a): 


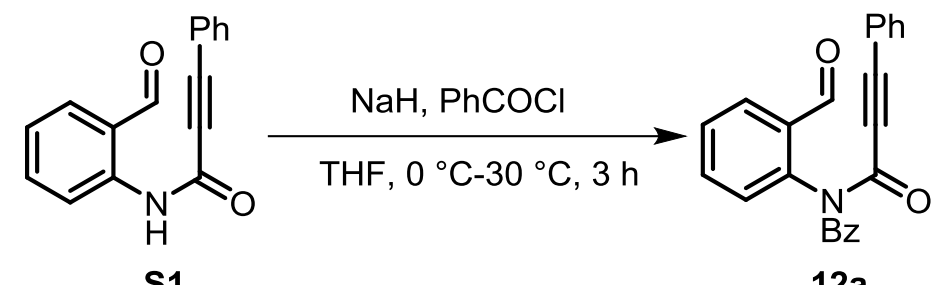

S1

$12 a$

The phenylpropiolamide $\mathbf{S 1}$ was prepared according to the reported procedure, ${ }^{2}$ and $\mathbf{S 1}$ (1.246 $\mathrm{g}, 5 \mathrm{mmol})$ in THF $(10 \mathrm{~mL})$ was added dropwise in $5 \mathrm{~min}$. to the solution of $\mathrm{NaH}(156 \mathrm{mg}, 6.5$ mmol, 1.3 equiv) in THF $(10 \mathrm{~mL})$ at $0{ }^{\circ} \mathrm{C}$. The mixture was stirred at $0{ }^{\circ} \mathrm{C}$ for 0.5 hour, then benzoyl chloride ( $0.7 \mathrm{~mL}, 6 \mathrm{mmol}, 1.2$ equiv) was added. The reaction mixture was stirred for 10 min. at $0{ }^{\circ} \mathrm{C}$, and allowed to $30{ }^{\circ} \mathrm{C}$ for $3 \mathrm{~h}$ (monitored by TLC), then quenched with saturated aqueous $\mathrm{Na}_{2} \mathrm{CO}_{3}$ solution $(5 \mathrm{~mL})$. The mixture was extracted with EtOAc, and the organic layer was washed with $\mathrm{H}_{2} \mathrm{O}$ and brine, dried over anhydrous $\mathrm{Na}_{2} \mathrm{SO}_{4}$, filtered, and concentrated under vacuum. The residue was purified by column chromatography on silica gel (eluent: Hexanes/EtOAc $=12 / 88)$ to afforded the corresponding products 12a as white solid $(1.20 \mathrm{gm}, 68 \%$ yield).

$\mathrm{R}_{f}=0.14(\mathrm{EA}: \mathrm{Hex}=1: 6) ; \mathrm{mp}=124.6-125.6^{\circ} \mathrm{C}$.

${ }^{1} \mathbf{H}$ NMR $\left(400 \mathrm{MHz}, \mathrm{CDCl}_{3}, 25^{\circ} \mathrm{C}\right) \delta / \mathrm{ppm}: 10.17$ (s, 1H), 8.01 (dd, $\left.J=7.5,1.7 \mathrm{~Hz}, 1 \mathrm{H}\right), 7.93-$ $7.88(\mathrm{~m}, 2 \mathrm{H}), 7.74(\mathrm{td}, J=7.4,1.8 \mathrm{~Hz}, 1 \mathrm{H}), 7.66(\mathrm{td}, J=7.4,1.8 \mathrm{~Hz}, 1 \mathrm{H}), 7.55(\mathrm{tt}, J=7.4,2.1 \mathrm{~Hz}$, $1 \mathrm{H}), 7.47(\mathrm{t}, J=7.7,2 \mathrm{H}), 7.45(\mathrm{dd}, J=7.7,1.2 \mathrm{~Hz}, 1 \mathrm{H}), 7.38(\mathrm{tt}, J=7.5,2.1 \mathrm{~Hz}, 1 \mathrm{H}), 7.26(\mathrm{t}, J=$ $7.5 \mathrm{~Hz}, 2 \mathrm{H}), 7.12(\mathrm{dd}, J=8.5,1.3 \mathrm{~Hz}, 2 \mathrm{H})$.

${ }^{13} \mathrm{C}$ NMR $\left(100 \mathrm{MHz}, \mathrm{CDCl}_{3}, 25{ }^{\circ} \mathrm{C}\right) \delta / \mathrm{ppm}: 189.6,171.6,154.3,138.4,134.7,133.2,132.9,132.7$, 132.6, 131.2, 130.9, 129.7, 129.4, 128.44, 128.43, 119.1, 96.0, 82.6.

IR $(\mathrm{KBr}) \tilde{v}\left(\mathrm{~cm}^{-1}\right): 3650,2362,2207,1725,1696,1489,1264,1198,950,757$.

HRMS (ESI) $m / z:[\mathrm{M}+\mathrm{H}]^{+}$Calcd. for $\mathrm{C}_{23} \mathrm{H}_{16} \mathrm{NO}_{3} 354.1130$; found 354.1130.

HRMS (ESI) $m / z:[\mathrm{M}+\mathrm{Na}]^{+}$Calcd. for $\mathrm{C}_{23} \mathrm{H}_{15} \mathrm{NO}_{3} \mathrm{Na}$ 376.0950; found 376.0952 .

\section{3-Benzoyl-2-phenyl-4H-furo[3,2-c]chromen-4-one (4aa)}<smiles>O=C(c1ccccc1)c1c(-c2ccccc2)oc2c1c(=O)oc1ccccc12</smiles> 
Following the TP-2, 4aa was obtained from $1 \mathbf{a}(52.2 \mathrm{mg}, 0.3 \mathrm{mmol}), \mathrm{PPh}_{3}(86.6 \mathrm{mg}, 1.1$ equiv.), benzoyl chloride 3a (76.7 $\mu \mathrm{L}, 2.2$ equiv.) and DIPEA (78.4 $\mu \mathrm{L}, 1.5$ equiv.). The residue was purified by column chromatography $\left(\mathrm{SiO}_{2}\right.$, Hexanes/EtOAc= 90:10) to give as a white solid $(82.4$ $\mathrm{mg}, 75 \%$ yield); $\mathrm{R}_{f}=0.54$ (EA:Hex $\left.=2: 8\right) ; \mathrm{mp}=208.7-209.4{ }^{\circ} \mathrm{C}$.

${ }^{1} \mathbf{H}$ NMR $\left(400 \mathrm{MHz}, \mathrm{CDCl}_{3}, 25^{\circ} \mathrm{C}\right) \delta / \mathrm{ppm}: 8.00(\mathrm{~d}, J=7.8 \mathrm{~Hz}, 1 \mathrm{H}), 7.97(\mathrm{~d}, J=8.0 \mathrm{~Hz}, 2 \mathrm{H}), 7.74$ $-7.69(\mathrm{~m}, 2 \mathrm{H}), 7.56$ (q, J = 7.9 Hz, 2H), $7.48-7.39$ (m, 4H), $7.38-7.34$ (m, 3H).

${ }^{13} \mathrm{C} \mathrm{NMR}\left(100 \mathrm{MHz}, \mathrm{CDCl}_{3}, 25^{\circ} \mathrm{C}\right) \delta / \mathrm{ppm}: 190.5,156.5,156.2,154.1,153.0,136.8,134.1,131.3$, 129.9, 129.7, 128.9, 128.8, 128.1, 126.5, 124.7, 121.1, 118.3, 117.5, 112.3, 111.6.

IR (KBr) $\tilde{v}\left(\mathrm{~cm}^{-1}\right): 2920,2360,1731,1665,1487,1324,1227,1037,895,754$.

HRMS (ESI) $m / z:[\mathrm{M}+\mathrm{H}]^{+}$Calcd. for $\mathrm{C}_{24} \mathrm{H}_{15} \mathrm{O}_{4} 367.0970$; found 367.0973 .

General procedure for the gram scale preparation of furo[3,2-c] coumarin derivative 4aa

A dry and argon-flushed $100 \mathrm{~mL}$ round bottom flask equipped with a magnetic stir bar was sequentially charged with $1 \mathrm{a}(1.045 \mathrm{~g}, 6 \mathrm{mmol}), \mathrm{PPh}_{3}(1.73 \mathrm{~g}, 1.1$ equiv), anhydrous DCE (60 $\mathrm{mL}), \mathrm{PhCOCl} 3 \mathrm{a}(1.53 \mathrm{~mL}, 2.2$ equiv). and DIPEA ( $1.57 \mathrm{~mL}, 1.5$ equiv). The reaction mixture was stirred at $60{ }^{\circ} \mathrm{C}$ for 3 hours in an oil bath. After completion of the reaction, as observed by TLC analysis, solvent was removed in vacuo and the crude residue was subjected to flash column chromatography on silica gel to obtain the product $4 \mathbf{4 a a}$ (1.65 g, $75 \%$ yield).

\section{3-Benzoyl-8-bromo-2-phenyl-4H-furo[3,2-c] chromen-4-one (4ba)}

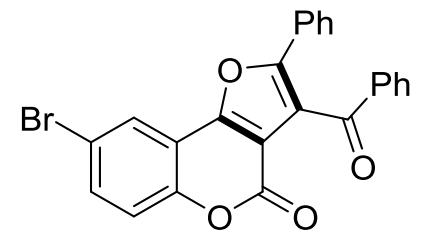

Following the TP-2, 4ba was obtained from $\mathbf{1 b}(75.6 \mathrm{mg}, 0.3 \mathrm{mmol}), \mathrm{PPh}_{3}$ (86.6 mg, 1.1 equiv.), benzoyl chloride 3a (76.7 $\mu \mathrm{L}, 2.2$ equiv.) and DIPEA ( $78.4 \mu \mathrm{L}, 1.5$ equiv.). The residue was purified by column chromatography $\left(\mathrm{SiO}_{2}, \mathrm{Hexanes} / \mathrm{EtOAc}=91: 9\right)$ to give as a white solid $(100.2$ $\mathrm{mg}, 75 \%$ yield); $\mathrm{R}_{f}=0.56$ (EA:Hex $\left.=2: 8\right) ; \mathrm{mp}=258.5-259.4{ }^{\circ} \mathrm{C}$.

${ }^{1} \mathbf{H}$ NMR $\left(400 \mathrm{MHz}, \mathrm{CDCl}_{3}, 25^{\circ} \mathrm{C}\right) \delta / \mathrm{ppm}: 8.14(\mathrm{~d}, J=2.3 \mathrm{~Hz}, 1 \mathrm{H}), 7.95(\mathrm{~d}, J=8.0 \mathrm{~Hz}, 2 \mathrm{H}), 7.71$ (dd, $J=7.3,3.6 \mathrm{~Hz}, 2 \mathrm{H}), 7.65(\mathrm{dd}, J=8.6,2.8 \mathrm{~Hz}, 1 \mathrm{H}), 7.59$ (t, $J=7.2 \mathrm{~Hz}, 1 \mathrm{H}), 7.44$ (t, $J=7.8$ $\mathrm{Hz}, 2 \mathrm{H}), 7.41-7.37(\mathrm{~m}, 3 \mathrm{H}), 7.34(\mathrm{~d}, J=8.8 \mathrm{~Hz}, 1 \mathrm{H})$. 
${ }^{13} \mathrm{C}$ NMR $\left(100 \mathrm{MHz}, \mathrm{CDCl}_{3}, 25{ }^{\circ} \mathrm{C}\right) \delta / \mathrm{ppm}: 190.2,155.8,154.73,154.70,151.7,136.7,134.3$, 134.1, 130.1, 129.7, 129.0, 128.8, 127.8, 126.6, 123.6, 119.2, 118.3, 117.6, 113.8, 112.3.

IR (KBr) $\tilde{v}\left(\mathrm{~cm}^{-1}\right): 2917,2342,1737,1663,1491,1299,976,732$.

HRMS (ESI) $m / z:[\mathrm{M}+\mathrm{H}]^{+}$Calcd. for $\mathrm{C}_{24} \mathrm{H}_{14}{ }^{79} \mathrm{BrO}_{4} 445.0075$; found 445.0076 .

HRMS (ESI) $m / z:[\mathrm{M}+\mathrm{H}]^{+}$Calcd. for $\mathrm{C}_{24} \mathrm{H}_{14}{ }^{81} \mathrm{BrO}_{4} 447.0055$; found 447.0061 .

3-Benzoyl-8-chloro-2-phenyl-4H-furo[3,2-c]chromen-4-one (4ca)

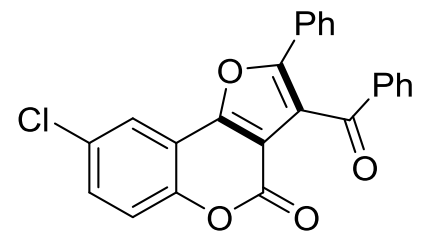

Following the TP-2, 4ca was obtained from $1 \mathbf{c}(62.4 \mathrm{mg}, 0.3 \mathrm{mmol}), \mathrm{PPh}_{3}$ ( $86.6 \mathrm{mg}, 1.1$ equiv.), benzoyl chloride 3a (76.7 $\mu \mathrm{L}, 2.2$ equiv.) and DIPEA (78.4 $\mu \mathrm{L}, 1.5$ equiv.). The residue was purified by column chromatography $\left(\mathrm{SiO}_{2}\right.$, Hexanes/EtOAc $\left.=92: 8\right)$ to give as a white solid $(85.4$ $\mathrm{mg}, 71 \%$ yield); $\mathrm{R}_{f}=0.67$ (EA:Hex $\left.=2: 8\right) ; \mathrm{mp}=243.4-244.0^{\circ} \mathrm{C}$.

${ }^{1} \mathbf{H}$ NMR $\left(400 \mathrm{MHz}, \mathrm{CDCl}_{3}, 25^{\circ} \mathrm{C}\right) \delta / \mathrm{ppm}: 7.99(\mathrm{~d}, J=2.6 \mathrm{~Hz}, 1 \mathrm{H}), 7.96(\mathrm{~d}, J=8.1 \mathrm{~Hz}, 2 \mathrm{H}), 7.76$ $-7.69(\mathrm{~m}, 2 \mathrm{H}), 7.59$ (t, $J=7.5 \mathrm{~Hz}, 1 \mathrm{H}), 7.51(\mathrm{dd}, J=8.1,2.3 \mathrm{~Hz}, 1 \mathrm{H}), 7.44(\mathrm{pt}, J=8.0 \mathrm{~Hz}, 2 \mathrm{H})$, $7.42-7.36(\mathrm{~m}, 4 \mathrm{H})$.

${ }^{13} \mathrm{C}$ NMR $\left(100 \mathrm{MHz}, \mathrm{CDCl}_{3}, 25{ }^{\circ} \mathrm{C}\right) \delta / \mathrm{ppm}: 190.2,155.9,154.9,154.7,151.2,136.7,134.3,131.2$, 130.4, 130.1, 129.7, 129.0, 128.8, 127.8, 126.6, 120.6, 118.9, 118.3, 113.3, 112.3.

IR (KBr) $\tilde{v}\left(\mathrm{~cm}^{-1}\right):$ 2950, 2320, 1750, 1667, 1495, 1230, 1107, 978, 891, 737.

HRMS (ESI) $m / z:[\mathrm{M}+\mathrm{H}]^{+}$Calcd. for $\mathrm{C}_{24} \mathrm{H}_{14}{ }^{35} \mathrm{ClO}_{4} 401.0581$; found 401.0576 .

HRMS (ESI) $m / z:[\mathrm{M}+\mathrm{H}]^{+}$Calcd. for $\mathrm{C}_{24} \mathrm{H}_{14}{ }^{37} \mathrm{ClO}_{4} 403.0551$; found 403.0555 .

\section{3-Benzoyl-8-fluoro-2-phenyl-4H-furo[3,2-c] chromen-4-one (4da)}

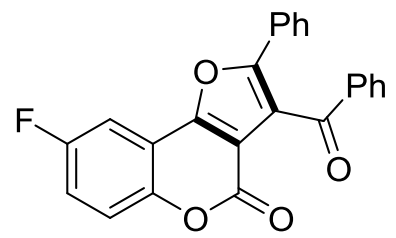

Following the TP-2, 4da was obtained from $1 \mathbf{1 d}(57.6 \mathrm{mg}, 0.3 \mathrm{mmol}), \mathrm{PPh}_{3}(86.6 \mathrm{mg}, 1.1$ equiv.), benzoyl chloride 3a (76.7 $\mu \mathrm{L}, 2.2$ equiv.) and DIPEA (78.4 $\mu \mathrm{L}, 1.5$ equiv.). The residue was purified by column chromatography $\left(\mathrm{SiO}_{2}, \mathrm{Hexanes} / \mathrm{EtOAc}=90: 10\right)$ to give as a white solid $(83.0$ $\mathrm{mg}, 72 \%$ yield); $\mathrm{R}_{f}=0.56$ (EA:Hex $\left.=2: 8\right) ; \mathrm{mp}=251.0-251.5^{\circ} \mathrm{C}$. 
${ }^{1} \mathrm{H}$ NMR $\left(400 \mathrm{MHz}, \mathrm{CDCl}_{3}, 25{ }^{\circ} \mathrm{C}\right) \delta / \mathrm{ppm}: 7.96(\mathrm{~d}, J=8.0 \mathrm{~Hz}, 2 \mathrm{H}), 7.75-7.66(\mathrm{~m}, 3 \mathrm{H}), 7.59(\mathrm{t}$, $J=7.3 \mathrm{~Hz}, 1 \mathrm{H}), 7.48-7.42(\mathrm{~m}, 3 \mathrm{H}), 7.38(\mathrm{t}, J=2.8 \mathrm{~Hz}, 3 \mathrm{H}), 7.29(\mathrm{dd}, J=8.5,2.8 \mathrm{~Hz}, 1 \mathrm{H})$.

${ }^{13} \mathrm{C}$ NMR $\left(100 \mathrm{MHz}, \mathrm{CDCl}_{3}, 25{ }^{\circ} \mathrm{C}\right) \delta / \mathrm{ppm}: 190.2,159.0\left(\mathrm{~d},{ }^{1} J_{\mathrm{C}-\mathrm{F}}=245.7 \mathrm{~Hz}\right), 156.1,155.29(\mathrm{~d}$, $\left.{ }^{4} J_{\mathrm{C}-\mathrm{F}}=2.7 \mathrm{~Hz}\right), 154.6,149.03,149.02,136.7,134.3,130.1,129.7,129.0,128.8,127.8,126.6$, $119.2\left(\mathrm{~d},{ }^{3} J_{\mathrm{C}-\mathrm{F}}=8.5 \mathrm{~Hz}\right), 118.7\left(\mathrm{~d},{ }^{2} J_{\mathrm{C}-\mathrm{F}}=24.4 \mathrm{~Hz}\right), 118.3,112.9\left(\mathrm{~d},{ }^{3} J_{\mathrm{C}-\mathrm{F}}=9.9 \mathrm{~Hz}\right), 112.3,106.9$ $\left(\mathrm{d},{ }^{2} J_{\mathrm{C}-\mathrm{F}}=25.5 \mathrm{~Hz}\right)$.

${ }^{19} \mathbf{F}$ NMR $\left(376 \mathrm{MHz}, \mathrm{CDCl} 3,25^{\circ} \mathrm{C}\right) \delta / \mathrm{ppm}:-115.87$

IR $(\mathrm{KBr}) \tilde{v}\left(\mathrm{~cm}^{-1}\right): 2943,2310,2098,1749,1664,1433,1082,892,753$.

HRMS (ESI) $m / z:[\mathrm{M}+\mathrm{H}]^{+}$Calcd. for $\mathrm{C}_{24} \mathrm{H}_{14} \mathrm{FO}_{4} 385.0876$; found 385.0880 .

3-Benzoyl-8-methyl-2-phenyl-4H-furo[3,2-c]chromen-4-one (4ea)<smiles>COc1ccc2oc(=O)c3c(C(=O)c4ccccc4)c(-c4ccccc4)oc3c2c1</smiles>

Following the TP-2, 4ea was obtained from $1 \mathrm{e}(56.4 \mathrm{mg}, 0.3 \mathrm{mmol}), \mathrm{PPh}_{3}$ (86.6 mg, 1.1 equiv.), benzoyl chloride 3a (76.7 $\mu \mathrm{L}, 2.2$ equiv.) and DIPEA ( $78.4 \mu \mathrm{L}, 1.5$ equiv.). The residue was purified by column chromatography $\left(\mathrm{SiO}_{2}, \mathrm{Hexanes} / \mathrm{EtOAc}=90: 10\right)$ to give as a white solid $(84.0$ $\mathrm{mg}, 74 \%$ yield); $\mathrm{R}_{f}=0.46$ (EA:Hex $\left.=2: 8\right) ; \mathrm{mp}=248.9-249.4{ }^{\circ} \mathrm{C}$.

${ }^{1} \mathbf{H}$ NMR $\left(400 \mathrm{MHz}, \mathrm{CDCl}_{3}, 25{ }^{\circ} \mathrm{C}\right) \delta / \mathrm{ppm}: 7.97(\mathrm{dd}, J=8.0,1.5 \mathrm{~Hz}, 2 \mathrm{H}), 7.79(\mathrm{~s}, 1 \mathrm{H}), 7.75-$ $7.70(\mathrm{~m}, 2 \mathrm{H}), 7.58(\mathrm{tt}, J=7.5,1.9 \mathrm{~Hz}, 1 \mathrm{H}), 7.44(\mathrm{t}, J=7.8 \mathrm{~Hz}, 2 \mathrm{H}), 7.40-7.31(\mathrm{~m}, 5 \mathrm{H}), 2.50(\mathrm{~s}$, $3 \mathrm{H})$.

${ }^{13} \mathrm{C} \mathrm{NMR}\left(100 \mathrm{MHz}, \mathrm{CDCl}_{3}, 25^{\circ} \mathrm{C}\right) \delta / \mathrm{ppm}: 190.6,156.7,156.3,153.8,151.2,136.8,134.6,134.1$, $132.4,129.8,129.7,128.9,128.7,128.1,126.5,120.8,118.3,117.2,111.9,111.5,20.9$.

IR $(\mathrm{KBr}) \tilde{v}\left(\mathrm{~cm}^{-1}\right): 2910,2340,2084,1743,1653,1230,1037,892,751$.

HRMS (ESI) $m / z:[\mathrm{M}+\mathrm{H}]^{+}$Calcd. for $\mathrm{C}_{25} \mathrm{H}_{17} \mathrm{O}_{4}$ 381.1127; found 381.1127 .

3-Benzoyl-8-methoxy-2-phenyl-4H-furo[3,2-c] chromen-4-one (4fa)<smiles>COc1ccc2oc(=O)c3c(C(=O)c4ccccc4)c(-c4ccccc4)oc3c2c1</smiles> 
Following the TP-2, 4fa was obtained from $1 \mathbf{f}(61.2 \mathrm{mg}, 0.3 \mathrm{mmol}), \mathrm{PPh}_{3}$ (86.6 mg, 1.1 equiv.), benzoyl chloride 3a (76.7 $\mu \mathrm{L}, 2.2$ equiv.) and DIPEA (78.4 $\mu \mathrm{L}, 1.5$ equiv.). The residue was purified by column chromatography $\left(\mathrm{SiO}_{2}\right.$, Hexanes/EtOAc $\left.=88: 12\right)$ to give as a white solid $(96.3$ $\mathrm{mg}, 81 \%$ yield); $\mathrm{R}_{f}=0.37$ (EA:Hex $\left.=2: 8\right) ; \mathrm{mp}=261.7-262.8^{\circ} \mathrm{C}$.

${ }^{1} \mathbf{H}$ NMR $\left(400 \mathrm{MHz}, \mathrm{CDCl}_{3}, 25{ }^{\circ} \mathrm{C}\right) \delta / \mathrm{ppm}: 7.96(\mathrm{dd}, J=8.2,1.7 \mathrm{~Hz}, 2 \mathrm{H}), 7.76-7.70(\mathrm{~m}, 2 \mathrm{H})$, $7.58(\mathrm{tt}, J=7.4,1.8 \mathrm{~Hz}, 1 \mathrm{H}), 7.43(\mathrm{t}, J=7.7 \mathrm{~Hz}, 2 \mathrm{H}), 7.40(\mathrm{~d}, J=3.1 \mathrm{~Hz}, 1 \mathrm{H}), 7.39-7.37(\mathrm{~m}$, 2H), $7.36(\mathrm{~d}, J=2.4 \mathrm{~Hz}, 2 \mathrm{H}), 7.13$ (dd, $J=9.2,3.0 \mathrm{~Hz}, 1 \mathrm{H}), 3.94(\mathrm{~s}, 3 \mathrm{H})$.

${ }^{13} \mathrm{C} \mathrm{NMR}\left(100 \mathrm{MHz}, \mathrm{CDCl}_{3}, 25^{\circ} \mathrm{C}\right) \mathrm{\delta} / \mathrm{ppm}: 190.5,156.6,156.4,156.1,154.0,147.5,136.8,134.1$, 129.9, 129.7, 128.9, 128.8, 128.1, 126.6, 119.3, 118.7, 118.4, 112.5, 111.8, 103.1, 56.0.

IR (KBr) $\tilde{v}\left(\mathrm{~cm}^{-1}\right): 2915,2342,1742,1668,1421,1325,1228,1033,892,751$.

HRMS (ESI) $m / z:[\mathrm{M}+\mathrm{H}]^{+}$Calcd. for $\mathrm{C}_{25} \mathrm{H}_{17} \mathrm{O}_{5}$ 397.1076; found 397.1078.

\section{3-Benzoyl-7-methoxy-2-phenyl-4H-furo[3,2-c] chromen-4-one (4ga)}

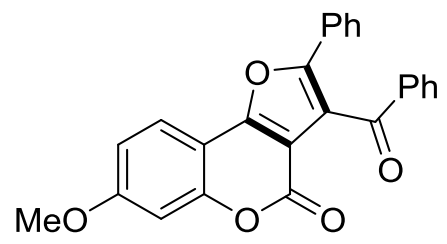

Following the TP-2, 4ga was obtained from $1 \mathrm{~g}$ (61.2 mg, $0.3 \mathrm{mmol}), \mathrm{PPh}_{3}(86.6 \mathrm{mg}, 1.1$ equiv.), benzoyl chloride 3a (76.7 $\mu \mathrm{L}, 2.2$ equiv.) and DIPEA ( $78.4 \mu \mathrm{L}, 1.5$ equiv.). The residue was purified by column chromatography $\left(\mathrm{SiO}_{2}\right.$, Hexanes/EtOAc= 85:15) to give as a white solid $(98.7$ $\mathrm{mg}, 83 \%$ yield); $\mathrm{R}_{f}=0.26$ (EA:Hex $\left.=2: 8\right) ; \mathrm{mp}=213.6-214.5^{\circ} \mathrm{C}$.

${ }^{1} \mathbf{H}$ NMR $\left(400 \mathrm{MHz}, \mathrm{CDCl}_{3}, 25{ }^{\circ} \mathrm{C}\right) \delta / \mathrm{ppm}: 7.97(\mathrm{dd}, J=8.2,1.4 \mathrm{~Hz}, 2 \mathrm{H}), 7.88(\mathrm{~d}, J=8.7 \mathrm{~Hz}$, 1H), $7.73-7.66(\mathrm{~m}, 2 \mathrm{H}), 7.57(\mathrm{tt}, J=7.3,1.3 \mathrm{~Hz}, 1 \mathrm{H}), 7.43(\mathrm{t}, J=8.0 \mathrm{~Hz}, 2 \mathrm{H}), 7.39-7.30(\mathrm{~m}$, $3 \mathrm{H}), 6.98(\mathrm{dd}, J=8.6,2.7 \mathrm{~Hz}, 1 \mathrm{H}), 6.92(\mathrm{~d}, J=2.5 \mathrm{~Hz}, 1 \mathrm{H}), 3.88(\mathrm{~s}, 3 \mathrm{H})$.

${ }^{13} \mathrm{C}$ NMR $\left(100 \mathrm{MHz}, \mathrm{CDCl}_{3}, 25{ }^{\circ} \mathrm{C}\right) \delta / \mathrm{ppm}: 190.6,162.5,156.9,156.7,154.8,153.2,136.9,134.0$, 129.66, 129.59, 128.9, 128.7, 128.2, 126.4, 122.1, 118.1, 113.1, 109.2, 105.5, 101.5, 55.8.

IR $(\mathrm{KBr}) \tilde{v}\left(\mathrm{~cm}^{-1}\right): 2950,2362,1745,1666,1450,1172,1029,893,751$.

HRMS (ESI) $m / z:[\mathrm{M}+\mathrm{H}]^{+}$Calcd. for $\mathrm{C}_{25} \mathrm{H}_{17} \mathrm{O}_{5} 397.1076$; found 397.1080.

3-Benzoyl-6-methoxy-2-phenyl-4H-furo[3,2-c]chromen-4-one (4ha) 


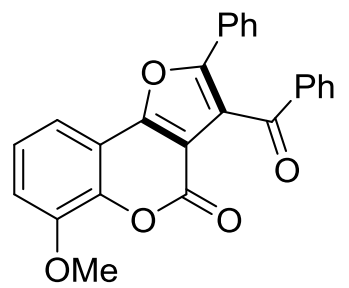

Following the TP-2, 4ha was obtained from $1 \mathbf{h}(61.2 \mathrm{mg}, 0.3 \mathrm{mmol}), \mathrm{PPh}_{3}$ ( $86.6 \mathrm{mg}, 1.1$ equiv.), benzoyl chloride 3a (76.7 $\mu \mathrm{L}, 2.2$ equiv.) and DIPEA (78.4 $\mu \mathrm{L}, 1.5$ equiv.). The residue was purified by column chromatography $\left(\mathrm{SiO}_{2}, \mathrm{Hexanes} / \mathrm{EtOAc}=85: 15\right)$ to give as a white solid $(95.1$ $\mathrm{mg}, 80 \%$ yield); $\mathrm{R}_{f}=0.28(\mathrm{EA}: \mathrm{Hex}=2: 8) ; \mathrm{mp}=233.3-234.3^{\circ} \mathrm{C}$.

${ }^{1} \mathbf{H}$ NMR $\left(400 \mathrm{MHz}, \mathrm{CDCl}_{3}, 25^{\circ} \mathrm{C}\right) \delta / \mathrm{ppm}: 7.95(\mathrm{dd}, J=8.1,1.3 \mathrm{~Hz}, 2 \mathrm{H}), 7.72(\mathrm{dd}, J=7.4,1.8$ $\mathrm{Hz}, 2 \mathrm{H}), 7.61-7.54(\mathrm{~m}, 2 \mathrm{H}), 7.42(\mathrm{t}, J=7.8 \mathrm{~Hz}, 2 \mathrm{H}), 7.39-7.35(\mathrm{~m}, 3 \mathrm{H}), 7.32(\mathrm{~d}, J=7.9 \mathrm{~Hz}$, $1 \mathrm{H}), 7.10(\mathrm{dd}, J=8.0,1.3 \mathrm{~Hz}, 1 \mathrm{H}), 3.96(\mathrm{~s}, 3 \mathrm{H})$.

${ }^{13} \mathrm{C}$ NMR $\left(100 \mathrm{MHz}, \mathrm{CDCl}_{3}, 25{ }^{\circ} \mathrm{C}\right) \delta / \mathrm{ppm}: 190.4,156.3,155.8,154.1,147.8,142.7,136.8,134.1$, $129.8,129.7,128.9,128.7,128.1,126.5,124.8,118.3,113.3,112.9,112.4,111.7,56.3$.

IR $(\mathrm{KBr}) \tilde{v}\left(\mathrm{~cm}^{-1}\right): 2938,2362,1744,1663,1471,1274,1065,897,750$.

HRMS (ESI) $m / z:[\mathrm{M}+\mathrm{H}]^{+}$Calcd. for $\mathrm{C}_{25} \mathrm{H}_{17} \mathrm{O}_{5}$ 397.1076; found 397.1076.

\section{3-Benzoyl-2-phenyl-4H-benzo[f]furo[3,2-c]chromen-4-one (4ia)}

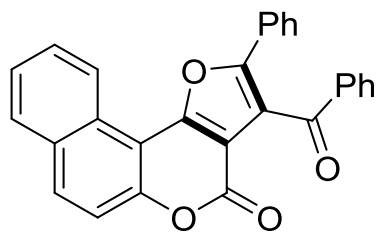

Following the TP-2, 4ia was obtained from $1 \mathbf{i}(67.2 \mathrm{mg}, 0.3 \mathrm{mmol}), \mathrm{PPh}_{3}(86.6 \mathrm{mg}, 1.1$ equiv.), benzoyl chloride 3a (76.7 $\mu \mathrm{L}, 2.2$ equiv.) and DIPEA (78.4 $\mu \mathrm{L}, 1.5$ equiv.). The residue was purified by column chromatography $\left(\mathrm{SiO}_{2}\right.$, Hexanes/EtOAc= 90:10) to give as a white solid $(28.7$ $\mathrm{mg}, 23 \%$ yield); $\mathrm{R}_{f}=0.54$ (EA:Hex $\left.=2: 8\right) ; \mathrm{mp}=298.5-299.4{ }^{\circ} \mathrm{C}$.

${ }^{1} \mathbf{H}$ NMR $\left(400 \mathrm{MHz}, \mathrm{CDCl}_{3}, 25{ }^{\circ} \mathrm{C}\right) \delta / \mathrm{ppm}: 9.12(\mathrm{~d}, J=8.5 \mathrm{~Hz}, 1 \mathrm{H}), 8.02(\mathrm{t}, J=4.4 \mathrm{~Hz}, 2 \mathrm{H}), 7.99$ $(\mathrm{d}, J=8.1 \mathrm{~Hz}, 2 \mathrm{H}), 7.85(\mathrm{pt}, J=7.8 \mathrm{~Hz}, 1 \mathrm{H}), 7.82(\mathrm{~d}, J=7.2 \mathrm{~Hz}, 2 \mathrm{H}), 7.66(\mathrm{t}, J=7.7 \mathrm{~Hz}, 1 \mathrm{H})$, 7.60 (pt, $J=7.2 \mathrm{~Hz}, 1 \mathrm{H}), 7.58$ (d, $J=8.4 \mathrm{~Hz}, 1 \mathrm{H}), 7.50-7.40$ (m, 5H).

${ }^{13} \mathrm{C}$ NMR $\left(100 \mathrm{MHz}, \mathrm{CDCl}_{3}, 25{ }^{\circ} \mathrm{C}\right) \delta / \mathrm{ppm}: 190.7,157.6,156.6,154.0,153.1,136.9,134.2,132.5$, $130.5,129.9,129.7,129.1,129.02,128.97,128.8,128.2,127.1,126.53,126.45,125.0,118.0$, $117.4,112.0,106.9$. 
IR $(\mathrm{KBr}) \tilde{v}\left(\mathrm{~cm}^{-1}\right): 2982,2362,1746,1669,1324,1225,1005,895,746$.

HRMS (ESI) $m / z:[\mathrm{M}+\mathrm{H}]^{+}$Calcd. for $\mathrm{C}_{28} \mathrm{H}_{17} \mathrm{O}_{4} 417.1127$; found 417.1130.

3-(4-Bromobenzoyl)-2-(4-bromophenyl)-4H-furo[3,2-c]chromen-4-one (4ab)

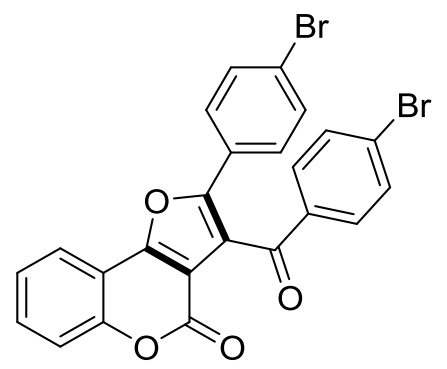

Following the TP-2, 4ab was obtained from $1 \mathbf{a}(52.2 \mathrm{mg}, 0.3 \mathrm{mmol}), \mathrm{PPh}_{3}(86.6 \mathrm{mg}, 1.1$ equiv.), 4-bromobenzoyl chloride $3 \mathbf{b}$ (144.8 mg, 2.2 equiv.) and DIPEA (78.4 $\mu \mathrm{L}, 1.5$ equiv.). The residue was purified by column chromatography $\left(\mathrm{SiO}_{2}\right.$, Hexanes/EtOAc $\left.=92: 8\right)$ to give as a white solid (125.8 mg, 80\% yield); $\mathrm{R}_{f}=0.63$ (EA:Hex $\left.=2: 8\right) ; \mathrm{mp}=276.0-276.9^{\circ} \mathrm{C}$.

${ }^{1} \mathbf{H}$ NMR $\left(400 \mathrm{MHz}, \mathrm{CDCl}_{3}, 25^{\circ} \mathrm{C}\right) \delta / \mathrm{ppm}: 8.01(\mathrm{dd}, J=8.1,1.7 \mathrm{~Hz}, 1 \mathrm{H}), 7.80(\mathrm{dt}, J=8.5,1.7$ $\mathrm{Hz}, 2 \mathrm{H}), 7.63-7.57(\mathrm{~m}, 5 \mathrm{H}), 7.53(\mathrm{dt}, J=8.5,1.9 \mathrm{~Hz}, 2 \mathrm{H}), 7.46(\mathrm{~d}, J=8.2 \mathrm{~Hz}, 1 \mathrm{H}), 7.43(\mathrm{t}, J=$ $7.4 \mathrm{~Hz}, 1 \mathrm{H})$.

${ }^{13} \mathrm{C}$ NMR $\left(100 \mathrm{MHz}, \mathrm{CDCl}_{3}, 25{ }^{\circ} \mathrm{C}\right) \delta / \mathrm{ppm}: 189.3,156.5,156.3,153.3,153.1,135.6,132.3,132.2$, 131.7, 131.0, 129.8, 127.9, 126.8, 124.9, 124.6, 121.2, 118.3, 117.6, 112.0, 111.5.

IR $(\mathrm{KBr}) \tilde{v}\left(\mathrm{~cm}^{-1}\right):$ 2910, 2362, 1747, 1667, 1487, 1094, 898, 754.

HRMS (ESI) $m / z:[\mathrm{M}+\mathrm{H}]^{+}$Calcd. for $\mathrm{C}_{24} \mathrm{H}_{13}{ }^{79} \mathrm{Br}^{79} \mathrm{BrO}_{4}$ 522.9181; found 522.9194 .

HRMS (ESI) $m / z:[\mathrm{M}+\mathrm{H}]^{+}$Calcd. for $\mathrm{C}_{24} \mathrm{H}_{13}{ }^{79} \mathrm{Br}^{81} \mathrm{BrO}_{4}$ 524.9160; found 524.9172 .

HRMS (ESI) $m / z:[\mathrm{M}+\mathrm{H}]^{+}$Calcd. for $\mathrm{C}_{24} \mathrm{H}_{13}{ }^{81} \mathrm{Br}^{79} \mathrm{BrO}_{4}$ 524.9160; found 524.9172 .

HRMS (ESI) $m / z:[\mathrm{M}+\mathrm{H}]^{+}$Calcd. for $\mathrm{C}_{24} \mathrm{H}_{13}{ }^{81} \mathrm{Br}^{81} \mathrm{BrO}_{4}$ 526.9140; found 526.9149 .

3-(4-Chlorobenzoyl)-2-(4-chlorophenyl)-4H-furo[3,2-c]chromen-4-one (4ac)

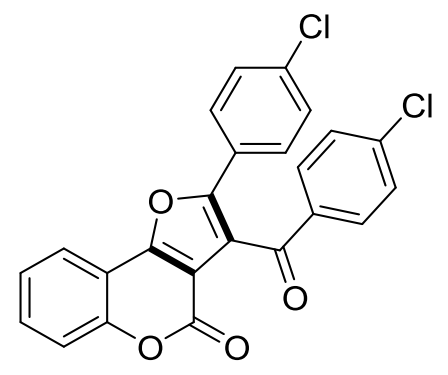

Following the TP-2, 4ac was obtained from $1 \mathrm{a}(52.2 \mathrm{mg}, 0.3 \mathrm{mmol}), \mathrm{PPh}_{3}(86.6 \mathrm{mg}, 1.1$ equiv.), 4-chlorobenzoyl chloride 3c ( $84.6 \mu \mathrm{L}, 2.2$ equiv.) and DIPEA (78.4 $\mu \mathrm{L}, 1.5$ equiv.). The residue 
was purified by column chromatography $\left(\mathrm{SiO}_{2}\right.$, Hexanes/EtOAc= 91:9) to give as a white solid (112.3 mg, 86\% yield); $\mathrm{R}_{f}=0.56(\mathrm{EA}: \mathrm{Hex}=2: 8) ; \mathrm{mp}=263.1-264.0{ }^{\circ} \mathrm{C}$.

${ }^{1} \mathbf{H}$ NMR $\left(400 \mathrm{MHz} \mathrm{CDCl}_{3}, 25{ }^{\circ} \mathrm{C}\right) \delta / \mathrm{ppm}: 8.00(\mathrm{dd}, J=7.9,1.7 \mathrm{~Hz}, 1 \mathrm{H}), 7.88(\mathrm{dt}, J=8.5,1.9$ $\mathrm{Hz}, 2 \mathrm{H}), 7.66(\mathrm{dt}, J=8.5,1.8 \mathrm{~Hz}, 2 \mathrm{H}), 7.59$ (td, $J=7.8,1.8 \mathrm{~Hz}, 1 \mathrm{H}), 7.46(\mathrm{~d}, J=8.6 \mathrm{~Hz}, 1 \mathrm{H})$, $7.42(\mathrm{~d}, J=8.5 \mathrm{~Hz}, 3 \mathrm{H}), 7.37(\mathrm{dt}, J=8.7,2.0 \mathrm{~Hz}, 2 \mathrm{H})$.

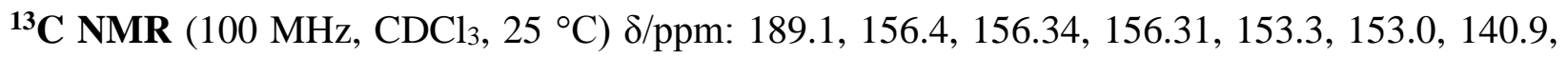
136.2 , 135.14, 135.12, 131.7, 131.5, 130.9, 129.3, 129.2, 128.9, 127.8, 126.4, 124.9, 121.2, 118.3, 117.6, 112.0, 111.4 .

IR $(\mathrm{KBr}) \tilde{v}\left(\mathrm{~cm}^{-1}\right):$ 2917, 2389, 2098, 1736, 1658, 1484, 1093, 895, 757.

HRMS (ESI) $m / z:[\mathrm{M}+\mathrm{H}]^{+}$Calcd. for $\mathrm{C}_{24} \mathrm{H}_{13}{ }^{35} \mathrm{Cl}^{35} \mathrm{ClO}_{4} 435.0191$; found 435.0190 .

HRMS (ESI) $m / z:[\mathrm{M}+\mathrm{H}]^{+}$Calcd. for $\mathrm{C}_{24} \mathrm{H}_{13}{ }^{35} \mathrm{Cl}^{37} \mathrm{ClO}_{4} 437.0161$; found 437.0165.

HRMS (ESI) $m / z:[\mathrm{M}+\mathrm{H}]^{+}$Calcd. for $\mathrm{C}_{24} \mathrm{H}_{13}{ }^{37} \mathrm{Cl}^{35} \mathrm{ClO}_{4} 437.0161$; found 437.0165.

HRMS (ESI) $m / z:[\mathrm{M}+\mathrm{H}]^{+}$Calcd. for $\mathrm{C}_{24} \mathrm{H}_{13}{ }^{37} \mathrm{Cl}^{37} \mathrm{ClO}_{4} 439.0132$; found 439.0147 .

3-(4-Fluorobenzoyl)-2-(4-fluorophenyl)-4H-furo[3,2-c]chromen-4-one (4ad)

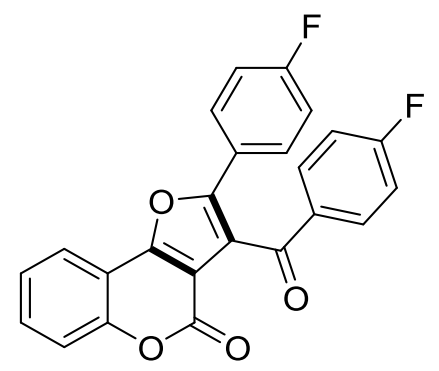

Following the TP-2, 4ad was obtained from $1 \mathbf{a}(52.2 \mathrm{mg}, 0.3 \mathrm{mmol}), \mathrm{PPh}_{3}$ (86.6 mg, 1.1 equiv.), 4-fluorobenzoyl chloride 3d (78.0 $\mu \mathrm{L}, 2.2$ equiv.) and DIPEA (78.4 $\mu \mathrm{L}, 1.5$ equiv.). The residue was purified by column chromatography $\left(\mathrm{SiO}_{2}, \mathrm{Hexanes} / \mathrm{EtOAc}=90: 10\right)$ to give as a white solid (96.6 mg, 80\% yield); $\mathrm{R}_{f}=0.47(\mathrm{EA}: \mathrm{Hex}=2: 8) ; \mathrm{mp}=224.7-225.6{ }^{\circ} \mathrm{C}$.

${ }^{1} \mathbf{H}$ NMR (400 MHz, $\left.\mathrm{CDCl}_{3}, 25^{\circ} \mathrm{C}\right) \delta / \mathrm{ppm}: 8.00$ (t, $\left.J=7.6 \mathrm{~Hz}, 2 \mathrm{H}\right), 7.97(\mathrm{~d}, J=8.0 \mathrm{~Hz}, 1 \mathrm{H}), 7.78$ $-7.70(\mathrm{~m}, 2 \mathrm{H}), 7.59(\mathrm{td}, J=7.6,1.5 \mathrm{~Hz}, 1 \mathrm{H}), 7.47(\mathrm{~d}, J=8.2 \mathrm{~Hz}, 1 \mathrm{H}), 7.43(\mathrm{t}, J=7.7 \mathrm{~Hz}, 1 \mathrm{H})$, $7.11(\mathrm{q}, J=7.9 \mathrm{~Hz}, 4 \mathrm{H})$.

${ }^{13} \mathrm{C}$ NMR $\left(100 \mathrm{MHz}, \mathrm{CDCl}_{3}, 25{ }^{\circ} \mathrm{C}\right) \delta / \mathrm{ppm}: 188.7,166.4\left(\mathrm{~d},{ }^{1} J_{\mathrm{C}-\mathrm{F}}=258.0 \mathrm{~Hz}\right), 163.6\left(\mathrm{~d},{ }^{1} J_{\mathrm{C}-\mathrm{F}}=\right.$ $251.7 \mathrm{~Hz}), 156.4,156.3,153.5,153.0,133.38\left(\mathrm{~d},{ }^{4} J_{\mathrm{C}-\mathrm{F}}=2.7 \mathrm{~Hz}\right), 132.35\left(\mathrm{~d},{ }^{3} J_{\mathrm{C}-\mathrm{F}}=9.6 \mathrm{~Hz}\right), 131.5$, $128.8\left(\mathrm{~d},{ }^{3} J_{\mathrm{C}-\mathrm{F}}=8.6 \mathrm{~Hz}\right), 124.8,124.3\left(\mathrm{~d},{ }^{4} J_{\mathrm{C}-\mathrm{F}}=2.8 \mathrm{~Hz}\right), 121.1,117.8,117.5,116.3\left(\mathrm{~d},{ }^{2} J_{\mathrm{C}-\mathrm{F}}=\right.$ $20.0 \mathrm{~Hz}), 116.1\left(\mathrm{~d},{ }^{2} J_{\mathrm{C}-\mathrm{F}}=19.7 \mathrm{~Hz}\right)$. 
${ }^{19}$ F NMR (376 MHz, $\left.\mathrm{CDCl}_{3}, 25^{\circ} \mathrm{C}\right) \delta / \mathrm{ppm}:-102.87,-109.14$.

IR $(\mathrm{KBr}) \tilde{v}\left(\mathrm{~cm}^{-1}\right):$ 2938, 2362, 1745, 1665, 1499, 1233, 1033, 855, 754.

HRMS (ESI) $m / z$ : $[\mathrm{M}+\mathrm{H}]^{+}$Calcd. for $\mathrm{C}_{24} \mathrm{H}_{13} \mathrm{~F}_{2} \mathrm{O}_{4}$ 403.0782; found 403.0785.

3-(4-Methylbenzoyl)-2-(p-tolyl)-4H-furo[3,2-c]chromen-4-one (4ae)

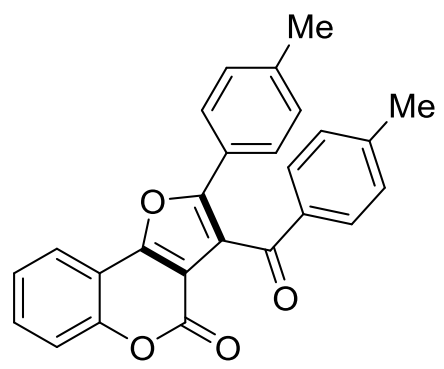

Following the TP-2, 4ae was obtained from $1 \mathbf{a}(52.2 \mathrm{mg}, 0.3 \mathrm{mmol}), \mathrm{PPh}_{3}$ (86.6 mg, 1.1 equiv.), 4-methylbenzoyl chloride $3 \mathbf{e}(87.9 \mu \mathrm{L}, 2.2$ equiv.) and DIPEA (78.4 $\mu \mathrm{L}, 1.5$ equiv.). The residue was purified by column chromatography $\left(\mathrm{SiO}_{2}\right.$, Hexanes/EtOAc= 88:12) to give as a white solid (79.3 mg, 67\% yield); $\mathrm{R}_{f}=0.43(\mathrm{EA}: \mathrm{Hex}=2: 8) ; \mathrm{mp}=252.8-253.8{ }^{\circ} \mathrm{C}$.

${ }^{1} \mathbf{H}$ NMR (400 MHz, $\left.\mathrm{CDCl}_{3}, 25^{\circ} \mathrm{C}\right) \delta / \mathrm{ppm}: 8.00(\mathrm{dd}, J=8.0,1.7 \mathrm{~Hz}, 1 \mathrm{H}), 7.86(\mathrm{~d}, J=8.1 \mathrm{~Hz}$, $2 \mathrm{H}), 7.60(\mathrm{~d}, J=8.3 \mathrm{~Hz}, 2 \mathrm{H}), 7.55(\mathrm{td}, J=8.0,1.4 \mathrm{~Hz}, 1 \mathrm{H}), 7.44(\mathrm{~d}, J=8.2 \mathrm{~Hz}, 1 \mathrm{H}), 7.40(\mathrm{td}, J=$ 7.5, 1.1 Hz, 1H), $7.23(\mathrm{~d}, J=8.0 \mathrm{~Hz}, 2 \mathrm{H}), 7.17(\mathrm{~d}, J=8.0 \mathrm{~Hz}, 2 \mathrm{H}), 2.39$ (s, 3H), 2.34 (s, 3H).

${ }^{13} \mathrm{C}$ NMR $\left(100 \mathrm{MHz}, \mathrm{CDCl}_{3}, 25{ }^{\circ} \mathrm{C}\right) \delta / \mathrm{ppm}: 190.2,156.5,155.9,154.1,152.9,145.1,140.1,134.5$, 131.1, 129.8, 129.6, 129.5, 126.4, 125.4, 124.6, 121.0, 117.7, 117.4, 112.3, 111.7, 21.8, 21.4.

IR $(\mathrm{KBr}) \tilde{v}\left(\mathrm{~cm}^{-1}\right): 2917,2310,1743,1656,1499,1237,1035,897,761$.

HRMS (ESI) $m / z$ : [M+H] $]^{+}$Calcd. for $\mathrm{C}_{26} \mathrm{H}_{19} \mathrm{O}_{4}$ 395.1283; found 395.1282.

3-(4-Methoxybenzoyl)-2-(4-methoxyphenyl)-4H-furo[3,2-c]chromen-4-one (4af)

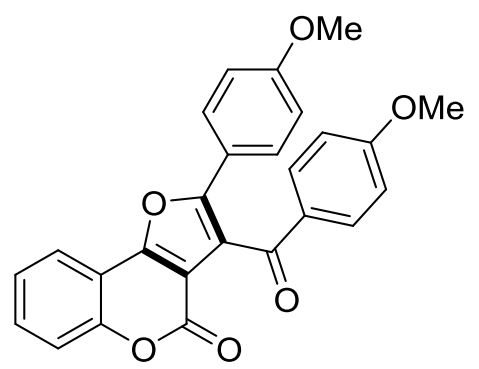

Following the TP-2, 4af was obtained from $1 \mathrm{a}(52.2 \mathrm{mg}, 0.3 \mathrm{mmol}), \mathrm{PPh}_{3}$ (86.6 mg, 1.1 equiv.), 4-methoxybenzoyl chloride $3 f(89.3 \mu \mathrm{L}, 2.2$ equiv.) and DIPEA (78.4 $\mu \mathrm{L}, 1.5$ equiv.). The residue was purified by column chromatography $\left(\mathrm{SiO}_{2}\right.$, Hexanes/EtOAc $\left.=85: 15\right)$ to give as a white solid (80.6 mg, 63\% yield); $\mathrm{R}_{f}=0.21(\mathrm{EA}: \mathrm{Hex}=2: 8) ; \mathrm{mp}=231.2-232.3{ }^{\circ} \mathrm{C}$. 
${ }^{1} \mathrm{H}$ NMR $\left(400 \mathrm{MHz}, \mathrm{CDCl}_{3}, 25{ }^{\circ} \mathrm{C}\right) \delta / \mathrm{ppm}: 7.99(\mathrm{dd}, J=7.8,1.3 \mathrm{~Hz}, 1 \mathrm{H}), 7.94(\mathrm{~d}, J=8.8 \mathrm{~Hz}$, 2H), $7.67(\mathrm{~d}, J=8.9 \mathrm{~Hz}, 2 \mathrm{H}), 7.55(\mathrm{td}, J=8.0,1.8 \mathrm{~Hz}, 1 \mathrm{H}), 7.45(\mathrm{~d}, J=8.3 \mathrm{~Hz}, 1 \mathrm{H}), 7.40(\mathrm{t}, J=$ $7.6 \mathrm{~Hz}, 1 \mathrm{H}), 6.90(\mathrm{~d}, J=4.5 \mathrm{~Hz}, 2 \mathrm{H}), 6.88(\mathrm{~d}, J=4.6 \mathrm{~Hz}, 2 \mathrm{H}), 3.85$ (s, 3H), 3.81 (s, 3H).

${ }^{13} \mathrm{C}$ NMR $\left(100 \mathrm{MHz}, \mathrm{CDCl}_{3}, 25{ }^{\circ} \mathrm{C}\right) \delta / \mathrm{ppm}: 189.1,164.4,160.8,156.6,155.7,154.1,152.9,132.2$, 131.0, 130.2, 128.1, 124.6, 120.98, 120.89, 117.4, 116.9, 114.4, 114.0, 112.4, 111.7, 55.5, 55.4.

IR $(\mathrm{KBr}) \tilde{v}\left(\mathrm{~cm}^{-1}\right): 2843,2344,1744,1654,1457,1259,1033,850,755$.

HRMS (ESI) $m / z:[\mathrm{M}+\mathrm{H}]^{+}$Calcd. for $\mathrm{C}_{26} \mathrm{H}_{19} \mathrm{O}_{6} 427.1182$; found 427.1179 .

3-(3-Chlorobenzoyl)-2-(3-chlorophenyl)-4H-furo[3,2-c]chromen-4-one (4ag)

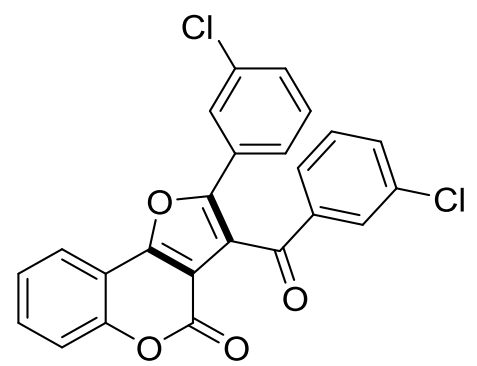

Following the TP-2, 4ag was obtained from $1 \mathbf{a}(52.2 \mathrm{mg}, 0.3 \mathrm{mmol}), \mathrm{PPh}_{3}$ (86.6 mg, 1.1 equiv.), 3-chlorobenzoyl chloride $3 \mathrm{~g}$ ( $84.5 \mu \mathrm{L}, 2.2$ equiv.) and DIPEA (78.4 $\mu \mathrm{L}, 1.5$ equiv.). The residue was purified by column chromatography $\left(\mathrm{SiO}_{2}\right.$, Hexanes/EtOAc $\left.=92: 8\right)$ to give as a white solid (104.5 mg, 80\% yield); $\mathrm{R}_{f}=0.58$ (EA:Hex $\left.=2: 8\right) ; \mathrm{mp}=192.8-193.7^{\circ} \mathrm{C}$.

${ }^{1} \mathbf{H}$ NMR $\left(400 \mathrm{MHz}, \mathrm{CDCl}_{3}, 25^{\circ} \mathrm{C}\right) \delta / \mathrm{ppm}: 8.03(\mathrm{dd}, J=8.0,1.6 \mathrm{~Hz}, 1 \mathrm{H}), 7.92(\mathrm{t}, J=1.9 \mathrm{~Hz}, 1 \mathrm{H})$, $7.83-7.77(\mathrm{~m}, 2 \mathrm{H}), 7.60(\mathrm{dd}, J=8.0,1.7 \mathrm{~Hz}, 1 \mathrm{H}), 7.55$ (tt, $J=7.0,1.4 \mathrm{~Hz}, 2 \mathrm{H}), 7.45(\mathrm{~d}, J=8.4$ $\mathrm{Hz}, 1 \mathrm{H}), 7.43$ (t, $J=7.6 \mathrm{~Hz}, 1 \mathrm{H}), 7.38$ (pt, $J=7.8 \mathrm{~Hz}, 1 \mathrm{H}), 7.37-7.34$ (m, 1H), 7.30 (t, $J=7.8$ $\mathrm{Hz}, 1 \mathrm{H})$

${ }^{13} \mathrm{C}$ NMR $\left(100 \mathrm{MHz}, \mathrm{CDCl}_{3}, 25{ }^{\circ} \mathrm{C}\right) \delta / \mathrm{ppm}: 188.9,156.6,156.2,153.1,152.8,138.3,135.17$, 135.12, 134.2, 131.8, 130.3, 130.13, 130.09, 129.5, 129.3, 127.8, 126.4, 124.9, 124.7, 121.3, 118.7, 117.5, 111.9, 111.4 .

IR (KBr) $\tilde{v}\left(\mathrm{~cm}^{-1}\right): 2938,2360,1744,1665,1420,1223,1036,856,744$.

HRMS (ESI) $m / z:[\mathrm{M}+\mathrm{H}]^{+}$Calcd. for $\mathrm{C}_{24} \mathrm{H}_{13}{ }^{35} \mathrm{Cl}^{35} \mathrm{ClO}_{4} 435.0191$; found 435.0190 .

HRMS (ESI) $m / z:[\mathrm{M}+\mathrm{H}]^{+}$Calcd. for $\mathrm{C}_{24} \mathrm{H}_{13}{ }^{35} \mathrm{Cl}^{37} \mathrm{ClO}_{4} 437.0161$; found 437.0164 .

HRMS (ESI) $m / z$ : $[\mathrm{M}+\mathrm{H}]^{+}$Calcd. for $\mathrm{C}_{24} \mathrm{H}_{13}{ }^{37} \mathrm{Cl}^{35} \mathrm{ClO}_{4} 437.0161$; found 437.0164 .

HRMS (ESI) $m / z:[\mathrm{M}+\mathrm{H}]^{+}$Calcd. for $\mathrm{C}_{24} \mathrm{H}_{13}{ }^{37} \mathrm{Cl}^{37} \mathrm{ClO}_{4} 439.0132$; found 439.0147.

3-(3-Methylbenzoyl)-2-(m-tolyl)-4H-furo[3,2-c]chromen-4-one (4ah) 


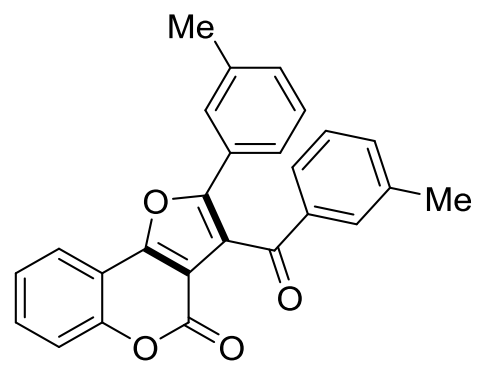

Following the TP-2, 4ah was obtained from 1a (52.2 mg, $0.3 \mathrm{mmol}), \mathrm{PPh}_{3}(86.6 \mathrm{mg}, 1.1$ equiv.), 3-methylbenzoyl chloride $3 \mathbf{h}(86.7 \mu \mathrm{L}, 2.2$ equiv.) and DIPEA (78.4 $\mu \mathrm{L}, 1.5$ equiv.). The residue was purified by column chromatography $\left(\mathrm{SiO}_{2}\right.$, Hexanes/EtOAc= 90:10) to give as a white solid (88.8 mg, 75\% yield); $\mathrm{R}_{f}=0.52(\mathrm{EA}: \mathrm{Hex}=2: 8) ; \mathrm{mp}=215.3-216.3{ }^{\circ} \mathrm{C}$.

${ }^{1} \mathbf{H}$ NMR $\left(400 \mathrm{MHz}, \mathrm{CDCl}_{3}, 25^{\circ} \mathrm{C}\right) \delta / \mathrm{ppm}: 8.04(\mathrm{dd}, J=7.8,1.4 \mathrm{~Hz}, 1 \mathrm{H}), 7.81(\mathrm{~s}, 1 \mathrm{H}), 7.71(\mathrm{~d}, J$ $=7.9 \mathrm{~Hz}, 1 \mathrm{H}), 7.57(\mathrm{ptd}, J=8.1,1.7 \mathrm{~Hz}, 1 \mathrm{H}), 7.55(\mathrm{~s}, 1 \mathrm{H}), 7.49(\mathrm{~d}, J=8.0 \mathrm{~Hz}, 1 \mathrm{H}), 7.46(\mathrm{~d}, J=$ $8.1 \mathrm{~Hz}, 1 \mathrm{H}), 7.43(\mathrm{~d}, J=8.0 \mathrm{~Hz}, 1 \mathrm{H}), 7.39(\mathrm{~d}, J=7.0 \mathrm{~Hz}, 1 \mathrm{H}), 7.30$ (t, $J=7.5 \mathrm{~Hz}, 1 \mathrm{H}), 7.25$ (t, $J$ $=7.5 \mathrm{~Hz}, 1 \mathrm{H}), 7.17(\mathrm{~d}, J=8.0 \mathrm{~Hz}, 1 \mathrm{H}), 2.37(\mathrm{~s}, 3 \mathrm{H}), 2.34(\mathrm{~s}, 3 \mathrm{H})$.

${ }^{13} \mathrm{C} \mathrm{NMR}\left(100 \mathrm{MHz}, \mathrm{CDCl}_{3}, 25^{\circ} \mathrm{C}\right) \delta / \mathrm{ppm}: 190.8,156.5,156.1,154.1,153.0,138.7,138.6,136.9$, $135.0,131.2,130.7,129.9,128.8,128.6,128.1,127.2,127.0,124.7,123.8,121.1,118.3,117.5$, 112.4, 111.7, 21.4, 21.3.

IR $(\mathrm{KBr}) \tilde{v}\left(\mathrm{~cm}^{-1}\right): 2950,2375,1476,1669,1473,1204,965,754$.

HRMS (ESI) $m / z:[\mathrm{M}+\mathrm{H}]^{+}$Calcd. for $\mathrm{C}_{26} \mathrm{H}_{19} \mathrm{O}_{4} 395.1283$; found 395.1280.

2-(Thiophen-2-yl)-3-(thiophene-2-carbonyl)-4H-furo[3,2-c]chromen-4-one (4ai)

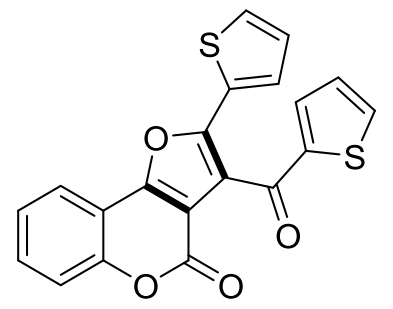

Following the TP-2, 4ai was obtained from $1 \mathbf{a}(52.2 \mathrm{mg}, 0.3 \mathrm{mmol}), \mathrm{PPh}_{3}(86.6 \mathrm{mg}, 1.1$ equiv.), 2-thiophenecarbonyl chloride 3i (70.6 $\mu \mathrm{L}, 2.2$ equiv.) and DIPEA (78.4 $\mu \mathrm{L}, 1.5$ equiv.). The residue was purified by column chromatography $\left(\mathrm{SiO}_{2}, \mathrm{Hexanes} / \mathrm{EtOAc}=88: 12\right)$ to give as a white solid (71.5 mg, 63\% yield); $\mathrm{R}_{f}=0.36(\mathrm{EA}: \mathrm{Hex}=2: 8) ; \mathrm{mp}=236.1-237.0^{\circ} \mathrm{C}$.

${ }^{1} \mathbf{H}$ NMR (400 MHz, $\left.\mathrm{CDCl}_{3}, 25^{\circ} \mathrm{C}\right) \delta / \mathrm{ppm}: 8.00(\mathrm{dd}, J=8.0,1.4 \mathrm{~Hz}, 1 \mathrm{H}), 7.75(\mathrm{~d}, J=5.0 \mathrm{~Hz}$, $1 \mathrm{H}), 7.61(\mathrm{dd}, J=3.9,1.4 \mathrm{~Hz}, 1 \mathrm{H}), 7.58(\mathrm{dd}, J=3.8,1.3 \mathrm{~Hz}, 1 \mathrm{H}), 7.57(\mathrm{dd}, J=7.1,1.7 \mathrm{~Hz}, 1 \mathrm{H})$, 
$7.46(\mathrm{~d}, J=8.4 \mathrm{~Hz}, 1 \mathrm{H}), 7.44(\mathrm{~d}, J=4.5 \mathrm{~Hz}, 1 \mathrm{H}), 7.40(\mathrm{~d}, J=7.7 \mathrm{~Hz}, 1 \mathrm{H}), 7.10(\mathrm{~d}, J=4.8 \mathrm{~Hz}$, $1 \mathrm{H}), 7.07(\mathrm{~d}, J=4.7 \mathrm{~Hz}, 1 \mathrm{H})$.

${ }^{13} \mathrm{C}$ NMR $\left(100 \mathrm{MHz}, \mathrm{CDCl}_{3}, 25{ }^{\circ} \mathrm{C}\right) \delta / \mathrm{ppm}: 181.1,156.3,155.9,153.0,150.7,143.9,135.7,135.2$, $131.4,129.5,128.6,128.4,128.3,128.1,124.8,121.2,117.4,116.8,112.0,111.0$.

IR $(\mathrm{KBr}) \tilde{v}\left(\mathrm{~cm}^{-1}\right): 2918,2350,1721,1659,1412,1095,829,751$.

HRMS (ESI) $m / z:[\mathrm{M}+\mathrm{H}]^{+}$Calcd. for $\mathrm{C}_{20} \mathrm{H}_{11} \mathrm{O}_{4} \mathrm{~S}_{2}$ 379.0099; found 379.0099 .

3-(Furan-2-carbonyl)-2-(furan-2-yl)-4H-furo[3,2-c]chromen-4-one (4aj)

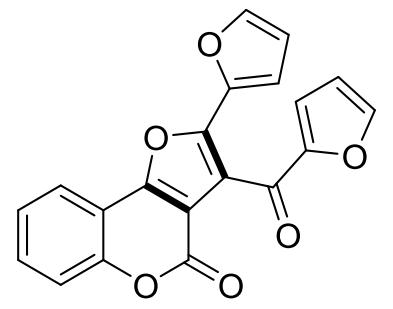

Following the TP-2, 4aj was obtained from $1 \mathrm{a}(52.2 \mathrm{mg}, 0.3 \mathrm{mmol}), \mathrm{PPh}_{3}$ ( $86.6 \mathrm{mg}, 1.1$ equiv.), 2-furoyl chloride $3 \mathbf{j}$ (64.8 $\mu \mathrm{L}, 2.2$ equiv.) and DIPEA (78.4 $\mu \mathrm{L}, 1.5$ equiv.). The residue was purified by column chromatography $\left(\mathrm{SiO}_{2}, \mathrm{Hexanes} / \mathrm{EtOAc}=85: 15\right)$ to give as a white solid $(67.5$ $\mathrm{mg}, 65 \%$ yield); $\mathrm{R}_{f}=0.25$ (EA:Hex $\left.=2: 8\right) ; \mathrm{mp}=251.7-252.6{ }^{\circ} \mathrm{C}$.

${ }^{1} \mathbf{H}$ NMR $\left(400 \mathrm{MHz}, \mathrm{CDCl}_{3}, 25{ }^{\circ} \mathrm{C}\right) \delta / \mathrm{ppm}: 8.00(\mathrm{dd}, J=7.8,1.5 \mathrm{~Hz}, 1 \mathrm{H}), 7.60(\mathrm{~d}, J=1.2 \mathrm{~Hz}$, $1 \mathrm{H}), 7.57(\mathrm{dd}, J=7.8,1.7 \mathrm{~Hz}, 1 \mathrm{H}), 7.53(\mathrm{~d}, J=1.3 \mathrm{~Hz}, 1 \mathrm{H}), 7.46(\mathrm{~d}, J=8.3 \mathrm{~Hz}, 1 \mathrm{H}), 7.41(\mathrm{t}, J=$ $7.9 \mathrm{~Hz}, 1 \mathrm{H}), 7.28(\mathrm{~d}, J=3.7 \mathrm{~Hz}, 1 \mathrm{H}), 7.08(\mathrm{~d}, J=3.5 \mathrm{~Hz}, 1 \mathrm{H}), 6.58(\mathrm{dd}, J=3.6,1.6 \mathrm{~Hz}, 1 \mathrm{H}), 6.53$ (dd, $J=3.6,1.6 \mathrm{~Hz}, 1 \mathrm{H})$.

${ }^{13} \mathrm{C}$ NMR $\left(100 \mathrm{MHz}, \mathrm{CDCl}_{3}, 25{ }^{\circ} \mathrm{C}\right) \delta / \mathrm{ppm}: 175.5,156.3,156.1,153.0,147.5,147.4,144.7,143.0$, $131.4,124.7,121.2,119.8,117.4,116.3,112.8,112.5,112.08,112.05,110.8$.

IR $(\mathrm{KBr}) \tilde{v}\left(\mathrm{~cm}^{-1}\right): 2918,2348,1731,1636,1319,1007,852,757$.

HRMS (ESI) $m / z$ : $[\mathrm{M}+\mathrm{H}]^{+}$Calcd. for $\mathrm{C}_{20} \mathrm{H}_{11} \mathrm{O}_{6}$ 347.0556; found 347.0559 .

\section{3-Butyryl-2-propyl-4H-furo[3,2-c]chromen-4-one (4ak)}

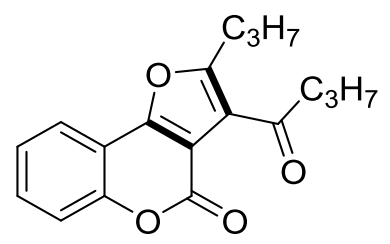

Following the TP-2, 4ak was obtained from 1a (52.2 mg, $0.3 \mathrm{mmol}), \mathrm{PPh}_{3}$ ( $86.6 \mathrm{mg}, 1.1$ equiv.), butyryl chloride 3k (68.2 $\mu \mathrm{L}, 2.2$ equiv.) and DIPEA (78.4 $\mu \mathrm{L}, 1.5$ equiv.). The residue was 
purified by column chromatography $\left(\mathrm{SiO}_{2}\right.$, Hexanes/EtOAc $\left.=95: 5\right)$ to give as a white solid $(60.9$ $\mathrm{mg}, 68 \%$ yield $) ; \mathrm{R}_{f}=0.79(\mathrm{EA}: \mathrm{Hex}=2: 8) ; \mathrm{mp}=90.0-90.9{ }^{\circ} \mathrm{C}$.

${ }^{1} \mathbf{H}$ NMR $\left(400 \mathrm{MHz} \mathrm{CDCl}_{3}, 25{ }^{\circ} \mathrm{C}\right) \delta / \mathrm{ppm}: 7.88(\mathrm{dd}, J=8.0,1.3 \mathrm{~Hz}, 1 \mathrm{H}), 7.55(\mathrm{td}, J=7.9,1.5$ $\mathrm{Hz}, 1 \mathrm{H}), 7.45(\mathrm{~d}, J=8.2 \mathrm{~Hz}, 1 \mathrm{H}), 7.37$ (t, $J=7.7 \mathrm{~Hz}, 1 \mathrm{H}), 3.22(\mathrm{t}, J=7.3 \mathrm{~Hz}, 2 \mathrm{H}), 2.99(\mathrm{t}, J=7.6$ $\mathrm{Hz}, 2 \mathrm{H}), 1.80$ (sext, $J=7.5 \mathrm{~Hz}, 2 \mathrm{H}), 1.71(\mathrm{sext}, J=7.5 \mathrm{~Hz}, 2 \mathrm{H}), 1.01$ (t, $J=7.5 \mathrm{~Hz}, 3 \mathrm{H}), 0.98$ (t, $J=7.5 \mathrm{~Hz}, 3 \mathrm{H})$.

${ }^{13} \mathrm{C}$ NMR $\left(100 \mathrm{MHz} \mathrm{CDCl}_{3}, 25^{\circ} \mathrm{C}\right) \delta / \mathrm{ppm}: 198.4,163.8,157.4,156.2,152.5,131.0,124.6,121.0$, 120.1, 117.1, 112.2, 108.0, 45.7, 29.4, 21.6, 17.7, 13.72, 13.67.

IR $(\mathrm{KBr}) \tilde{v}\left(\mathrm{~cm}^{-1}\right):$ 2961, 2364, 1756, 1671, 1393, 1086, 979, 895, 759.

HRMS (ESI) $m / z:[\mathrm{M}+\mathrm{H}]^{+}$Calcd. for $\mathrm{C}_{18} \mathrm{H}_{19} \mathrm{O}_{4}$ 299.1283; found 299.1282.

3-Acetyl-2-methyl-4H-furo[3,2-c]chromen-4-one (4al)

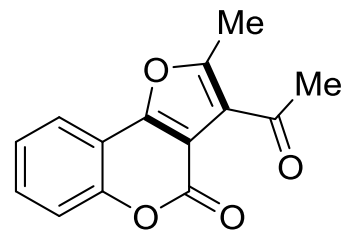

Following the TP-2, 4al was obtained from $1 \mathbf{a}(52.2 \mathrm{mg}, 0.3 \mathrm{mmol}), \mathrm{PPh} 3$ (86.6 mg, 1.1 equiv.), acetyl chloride $3 \mathrm{I}$ ( $46.9 \mu \mathrm{L}, 2.2$ equiv.) and DIPEA (78.4 $\mu \mathrm{L}, 1.5$ equiv.). The residue was purified by column chromatography $\left(\mathrm{SiO}_{2}, \mathrm{Hexanes} / \mathrm{EtOAc}=90: 10\right)$ to give as a white solid $(45.1 \mathrm{mg}, 62 \%$ yield); $\mathrm{R}_{f}=0.48(\mathrm{EA}: \mathrm{Hex}=2: 8) ; \mathrm{mp}=184.0-185.0{ }^{\circ} \mathrm{C}$.

${ }^{1} \mathbf{H}$ NMR $\left(400 \mathrm{MHz} \mathrm{CDCl}_{3}, 25{ }^{\circ} \mathrm{C}\right) \delta / \mathrm{ppm}: 7.86(\mathrm{dd}, J=8.0,1.3 \mathrm{~Hz}, 1 \mathrm{H}), 7.55(\mathrm{td}, J=7.8,1.5$ Hz, 1H), 7.44 (d, $J=8.3 \mathrm{~Hz}, 1 \mathrm{H}), 7.36$ (t, $J=7.6 \mathrm{~Hz}, 1 \mathrm{H}), 2.83$ (s, 3H), 2.69 (s, 3H).

${ }^{13} \mathrm{C}$ NMR $\left(100 \mathrm{MHz} \mathrm{CDCl}_{3}, 25^{\circ} \mathrm{C}\right) \delta / \mathrm{ppm}: 195.4,160.8,157.2,156.2,152.5,131.1,124.6,121.0$, 120.1, 117.0, 112.0, 108.0, 32.1, 14.1 .

IR $(\mathrm{KBr}) \tilde{v}\left(\mathrm{~cm}^{-1}\right): 2956,2357,1732,1674,1405,973,856,762$.

HRMS (ESI) $m / z:[\mathrm{M}+\mathrm{H}]^{+}$Calcd. for $\mathrm{C}_{14} \mathrm{H}_{11} \mathrm{O}_{4}$ 243.0657; found 243.0659.

3-(2,2,2-Trifluoro-1,1-dihydroxyethyl)-2-(trifluoromethyl)-4H-furo[3,2-c]chromen-4-one (8)<smiles>O=c1oc2ccccc2c2oc(C(F)(F)F)c(C(O)(O)C(F)(F)F)c12</smiles> 
Following the TP-2, 8 was obtained from $1 \mathbf{a}(52.2 \mathrm{mg}, 0.3 \mathrm{mmol}), \mathrm{PPh}_{3}$ (86.6 mg, 1.1 equiv.), trifluoroacetic anhydride 3m (104.2 $\mu \mathrm{L}, 2.5$ equiv.) and DIPEA (78.4 $\mu \mathrm{L}, 1.5$ equiv.). The residue was purified by column chromatography $\left(\mathrm{SiO}_{2}\right.$, Hexanes/EtOAc= 87:13) to give as a white solid (82.8 mg, 75\% yield); $\mathrm{R}_{f}=0.37$ (EA:Hex $\left.=2: 8\right) ; \mathrm{mp}=140.9-142.0^{\circ} \mathrm{C}$.

${ }^{1} \mathbf{H}$ NMR $\left(400 \mathrm{MHz}, \mathrm{CD}_{3} \mathrm{OD}, 25^{\circ} \mathrm{C}\right) \delta / \mathrm{ppm}: 8.56(\mathrm{dd}, J=7.9,1.8 \mathrm{~Hz}, 1 \mathrm{H}), 8.27(\mathrm{td}, J=8.0,1.9$ $\mathrm{Hz}, 1 \mathrm{H}), 8.14(\mathrm{~s}, 2 \mathrm{H}), 8.08(\mathrm{~d}, J=8.3 \mathrm{~Hz}, 1 \mathrm{H}), 8.04(\mathrm{td}, J=7.8,1.0 \mathrm{~Hz}, 1 \mathrm{H})$

${ }^{13} \mathrm{C}$ NMR $\left(100 \mathrm{MHz}, \mathrm{CD}_{3} \mathrm{OD}, 25{ }^{\circ} \mathrm{C}\right) \delta / \mathrm{ppm}: 180.4,178.6,173.2,163.1\left(\mathrm{q},{ }^{2} J_{\mathrm{C}-\mathrm{F}}=43.5 \mathrm{~Hz}\right)$, $153.4,146.0,145.6,143.1\left(\mathrm{q},{ }^{1} J_{\mathrm{C}-\mathrm{F}}=291.0 \mathrm{~Hz}\right), 141.9,138.7\left(\mathrm{q},{ }^{1} J_{\mathrm{C}-\mathrm{F}}=270.0 \mathrm{~Hz}\right), 137.5,131.4$, $129.2,111.4\left(\mathrm{q},{ }^{2} J_{\mathrm{C}-\mathrm{F}}=34.7 \mathrm{~Hz}\right)$.

${ }^{19}$ F NMR $\left(376 \mathrm{MHz}, \mathrm{CD}_{3} \mathrm{OD}, 25^{\circ} \mathrm{C}\right) \delta / \mathrm{ppm}:-58.7(\mathrm{q}, J=4.6 \mathrm{~Hz}),-84.9(\mathrm{q}, J=4.5 \mathrm{~Hz})$

IR (KBr) $\tilde{v}\left(\mathrm{~cm}^{-1}\right): 3261,2950,2364,2334,1702,1515,1169,1037,906,757$.

HRMS (ESI) $m / z:$ : $\mathrm{M}-\mathrm{H}]^{+}$Calcd. for $\mathrm{C}_{14} \mathrm{H}_{5} \mathrm{~F}_{6} \mathrm{O}_{5}$ 367.0041; found 367.0046.

\section{2,3-Diphenyl-4H-furo[3,2-c]chromen-4-one (6aa)}

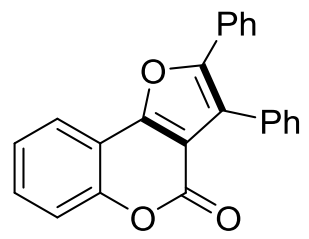

Following the TP-3, 6aa was obtained from 9a (75.1 mg, 0.3 mmol), $\mathrm{PPh}_{3}$ (86.6 mg, 1.1 equiv.), benzoyl chloride 3a (38.3 $\mu \mathrm{L}, 1.1$ equiv.) and DIPEA (78.4 $\mu \mathrm{L}, 1.5$ equiv.). The residue was purified by column chromatography $\left(\mathrm{SiO}_{2}, \mathrm{Hexanes} / \mathrm{DCM}=70: 30\right)$ to give as a white solid (80.2mg, 79\% yield); $\mathrm{R}_{f}=0.54$ (DCM:Hex $=2: 1$ ); $\mathrm{mp}=201.8-202.7^{\circ} \mathrm{C}$.

${ }^{1} \mathbf{H}$ NMR $\left(400 \mathrm{MHz}, \mathrm{CDCl}_{3}, 25^{\circ} \mathrm{C}\right) \delta / \mathrm{ppm}: 8.00(\mathrm{dd}, J=7.8,1.5 \mathrm{~Hz}, 1 \mathrm{H}), 7.59-7.49(\mathrm{~m}, 5 \mathrm{H})$, $7.48-7.41(\mathrm{~m}, 4 \mathrm{H}), 7.38(\mathrm{td}, J=7.5,0.9 \mathrm{~Hz}, 1 \mathrm{H}), 7.35-7.30(\mathrm{~m}, 3 \mathrm{H})$.

${ }^{13} \mathrm{C}$ NMR $\left(100 \mathrm{MHz}, \mathrm{CDCl}_{3}, 25{ }^{\circ} \mathrm{C}\right) \delta / \mathrm{ppm}: 157.5,156.4,152.7,151.4,130.7,130.22,130.18$, 129.3, 128.8, 128.59, 128.56, 128.4, 126.7, 124.4, 120.9, 117.2, 112.8, 111.3.

IR $(\mathrm{KBr}) \tilde{v}\left(\mathrm{~cm}^{-1}\right):$ 2950, 2350, 2014, 1742, 1093, 964, 745, 694.

HRMS (ESI) $m / z:[\mathrm{M}+\mathrm{H}]^{+}$Calcd. for $\mathrm{C}_{23} \mathrm{H}_{15} \mathrm{O}_{3} 339.1021$; found 339.1024 .

General procedure for the gram scale preparation of furo[3,2-c]coumarin derivative 6aa

A dry and argon-flushed $100 \mathrm{~mL}$ round bottom flask equipped with a magnetic stir bar was sequentially charged with 9a ( $1.0 \mathrm{~g}, 4 \mathrm{mmol}), \mathrm{PPh}_{3}(1.15 \mathrm{~g}, 1.1$ equiv), anhydrous DCE (40 mL), $\mathrm{PhCOCl} 3 \mathrm{a}(0.51 \mathrm{~mL}, 1.1$ equiv). and DIPEA ( $1.05 \mathrm{~mL}, 1.5$ equiv). The reaction mixture was 
stirred at $60{ }^{\circ} \mathrm{C}$ for 6 hours in an oil bath. After completion of the reaction, as observed by TLC analysis, solvent was removed in vасио and the crude residue was subjected to flash column chromatography on silica gel to obtain the product 6aa (1.06 g, 78\% yield).

\section{8-Bromo-2,3-diphenyl-4H-furo[3,2-c ]chromen-4-one (6ba)}<smiles>O=c1oc2ccc(Br)cc2c2oc(-c3ccccc3)c(-c3ccccc3)c12</smiles>

Following the TP-3, 6ba was obtained from 9b (98.7 mg, $0.3 \mathrm{mmol}), \mathrm{PPh}_{3}$ (86.6 mg, 1.1 equiv.), benzoyl chloride 3a (38.3 $\mu \mathrm{L}, 1.1$ equiv.) and DIPEA $(78.4 \mu \mathrm{L}, 1.5$ equiv.). The residue was purified by column chromatography $\left(\mathrm{SiO}_{2}\right.$, Hexanes/DCM=70:30) to give as a white solid (65.1mg, 52\% yield); $\mathrm{R}_{f}=0.55$ (DCM:Hex $\left.=2: 1\right) ; \mathrm{mp}=200.7-201.6{ }^{\circ} \mathrm{C}$.

${ }^{1} \mathbf{H}$ NMR $\left(400 \mathrm{MHz} \mathrm{CDCl}_{3}, 25^{\circ} \mathrm{C}\right) \delta / \mathrm{ppm}: 8.11(\mathrm{~d}, J=2.3 \mathrm{~Hz}, 1 \mathrm{H}), 7.61(\mathrm{dd}, J=8.5,2.2 \mathrm{~Hz}$, 1H), $7.57-7.52(\mathrm{~m}, 2 \mathrm{H}), 7.51-7.47(\mathrm{~m}, 2 \mathrm{H}), 7.47-7.42(\mathrm{~m}, 3 \mathrm{H}), 7.36-7.30(\mathrm{~m}, 4 \mathrm{H})$.

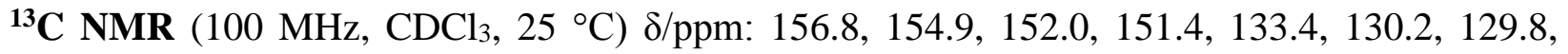
$129.05,128.98,128.63,128.60,128.5,126.7,123.4,120.9,118.9,117.2,114.3,112.0$.

IR $(\mathrm{KBr}) \tilde{v}\left(\mathrm{~cm}^{-1}\right): 2930,2363,1750,1443,1094,970,814,752$.

HRMS (ESI) $m / z:[\mathrm{M}+\mathrm{H}]^{+}$Calcd. for $\mathrm{C}_{23} \mathrm{H}_{14}{ }^{79} \mathrm{BrO}_{3} 417.0126$; found 417.0127.

HRMS (ESI) $m / z:[\mathrm{M}+\mathrm{H}]^{+}$Calcd. for $\mathrm{C}_{23} \mathrm{H}_{14}{ }^{81} \mathrm{BrO}_{3} 419.0106$; found 419.0108.

8-Methoxy-2,3-diphenyl-4H-furo[3,2-c]chromen-4-one (6ca)<smiles>COc1ccc2oc(=O)c3c(-c4ccccc4)c(-c4ccccc4)oc3c2c1</smiles>

Following the TP-3, 6ca was obtained from 9c $(84.1 \mathrm{mg}, 0.3 \mathrm{mmol}), \mathrm{PPh}_{3}$ (86.6 mg, 1.1 equiv.), benzoyl chloride 3a (38.3 $\mu \mathrm{L}, 1.1$ equiv.) and DIPEA (78.4 $\mu \mathrm{L}, 1.5$ equiv.). The residue was purified by column chromatography $\left(\mathrm{SiO}_{2}\right.$, Hexanes/DCM=60:40) to give as a white solid (79.6mg, 72\% yield); $\mathrm{R}_{f}=0.39$ (DCM:Hex $=2: 1$ ); $\mathrm{mp}=189.5-190.6{ }^{\circ} \mathrm{C}$.

${ }^{1} \mathbf{H}$ NMR $\left(400 \mathrm{MHz}, \mathrm{CDCl}_{3}, 25^{\circ} \mathrm{C}\right) \delta / \mathrm{ppm}: 7.58-7.53(\mathrm{~m}, 2 \mathrm{H}), 7.53-7.48(\mathrm{~m}, 2 \mathrm{H}), 7.47-7.41$ $(\mathrm{m}, 3 \mathrm{H}), 7.39(\mathrm{~s}, 1 \mathrm{H}), 7.37(\mathrm{~d}, J=5.5 \mathrm{~Hz}, 1 \mathrm{H}), 7.35-7.30(\mathrm{~m}, 3 \mathrm{H}), 7.10(\mathrm{dd}, J=8.5,3.0 \mathrm{~Hz}, 1 \mathrm{H})$, $3.94(\mathrm{~s}, 3 \mathrm{H})$. 
${ }^{13} \mathrm{C}$ NMR $\left(100 \mathrm{MHz}, \mathrm{CDCl}_{3}, 25{ }^{\circ} \mathrm{C}\right)$ d/ppm: 157.63, 157.59, 156.4, 156.2, 151.4, 147.2, 130.20, $130.16,129.3,128.8,128.6,128.5,128.4,126.8,121.0,118.8,118.4,113.0,111.5,102.86,102.82$, 56.0 .

IR $(\mathrm{KBr}) \tilde{v}\left(\mathrm{~cm}^{-1}\right): 2958,2360,1741,1421,1272,991,856,742,964$.

HRMS (ESI) $m / z:[\mathrm{M}+\mathrm{H}]^{+}$Calcd. for $\mathrm{C}_{24} \mathrm{H}_{17} \mathrm{O}_{4} 369.1127$; found 369.1128 .

2-(4-Chlorophenyl)-3-phenyl-4H-furo[3,2-c] chromen-4-one (6ac)

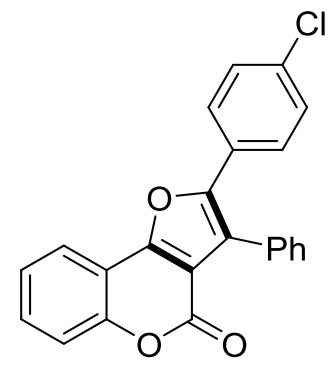

Following the TP-3, 6ac was obtained from 9a (75.1 mg, $0.3 \mathrm{mmol}), \mathrm{PPh}_{3}$ (86.6 mg, 1.1 equiv.), 4-chlorobenzoyl chloride 3c (42.3 $\mu \mathrm{L}, 1.1$ equiv.) and DIPEA (78.4 $\mu \mathrm{L}, 1.5$ equiv.). The residue was purified by column chromatography $\left(\mathrm{SiO}_{2}\right.$, Hexanes/DCM=65:35) to give as a white solid (90.6mg, 81\% yield); $\mathrm{R}_{f}=0.51$ (DCM:Hex $=2: 1$ ); $\mathrm{mp}=227.8-228.6^{\circ} \mathrm{C}$.

${ }^{1} \mathbf{H}$ NMR $\left(400 \mathrm{MHz}, \mathrm{CDCl}_{3}, 25^{\circ} \mathrm{C}\right) \delta / \mathrm{ppm}: 7.97(\mathrm{dd}, J=7.8,1.6 \mathrm{~Hz}, 1 \mathrm{H}), 7.53(\mathrm{td}, J=7.5,1.8$ $\mathrm{Hz}, 1 \mathrm{H}), 7.50-7.42$ (m, 8H), 7.38 (td, $J=7.7,1.3 \mathrm{~Hz}, 1 \mathrm{H}), 7.29$ (dt, $J=8.7,2.0 \mathrm{~Hz}, 2 \mathrm{H})$.

${ }^{13} \mathrm{C} \mathrm{NMR}\left(100 \mathrm{MHz}, \mathrm{CDCl}_{3}, 2{ }^{\circ} \mathrm{C}\right) \delta / \mathrm{ppm}: 157.3,156.5,152.7,150.2,134.7,130.9,130.1,129.8$, $128.9,128.7,128.6,127.8,127.7,124.5,121.4,120.9,117.3,112.6,111.3$.

IR $(\mathrm{KBr}) \tilde{v}\left(\mathrm{~cm}^{-1}\right): 2968,2359,1743,1505,1438,1093,967,832,750$.

HRMS (ESI) $m / z:[\mathrm{M}+\mathrm{H}]^{+}$Calcd. for $\mathrm{C}_{23} \mathrm{H}_{14}{ }^{35} \mathrm{ClO}_{3} 373.0631$; found 373.0637 .

HRMS (ESI) $m / z:[\mathrm{M}+\mathrm{H}]^{+} \mathrm{Calcd}$. for $\mathrm{C}_{23} \mathrm{H}_{14}{ }^{37} \mathrm{ClO}_{3} 375.0602$; found 375.0612 .

3-Phenyl-2-(p-tolyl)-4H-furo[3,2-c] chromen-4-one (6ae)

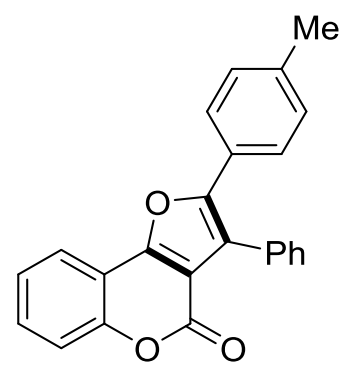

Following the TP-3, 6ae was obtained from 9a (75.1 mg, $0.3 \mathrm{mmol}$ ), $\mathrm{PPh}_{3}$ (86.6 mg, 1.1 equiv.), 4-methylbenzoyl chloride 3e (43.5 $\mu \mathrm{L}, 1.1$ equiv.) and DIPEA (78.4 $\mu \mathrm{L}, 1.5$ equiv.). The residue 
was purified by column chromatography $\left(\mathrm{SiO}_{2}\right.$, Hexanes/DCM=65:35) to give as a white solid (76.1mg, 72\% yield); $\mathrm{R}_{f}=0.46$ (DCM:Hex $=2: 1$ ); $\mathrm{mp}=229.5-230.4{ }^{\circ} \mathrm{C}$.

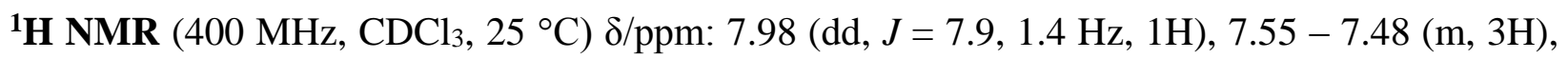
$7.47-7.40(\mathrm{~m}, 6 \mathrm{H}), 7.37(\mathrm{td}, J=7.4,1.5 \mathrm{~Hz}, 1 \mathrm{H}), 7.13(\mathrm{~d}, J=8.3 \mathrm{~Hz}, 2 \mathrm{H}), 2.35$ (s, 3H).

${ }^{13} \mathrm{C}$ NMR (100 MHz, $\left.\mathrm{CDCl}_{3}, 25{ }^{\circ} \mathrm{C}\right) \delta / \mathrm{ppm}: 157.56,157.51,156.2,152.6,151.7,139.0,130.5$, $130.31,130.25,129.3,128.5,128.3,126.6,126.5,124.4,120.8,120.2,117.2,112.8,111.3,21.3$. IR $(\mathrm{KBr}) \tilde{v}\left(\mathrm{~cm}^{-1}\right): 3021,2358,1743,1318,1088,965,821,754,701$.

HRMS (ESI) $m / z:[\mathrm{M}+\mathrm{H}]^{+}$Calcd. for $\mathrm{C}_{24} \mathrm{H}_{17} \mathrm{O}_{3} 353.1178$; found 353.1180.

\section{2-(Furan-2-yl)-3-phenyl-4H-furo[3,2-c]chromen-4-one (6aj)}

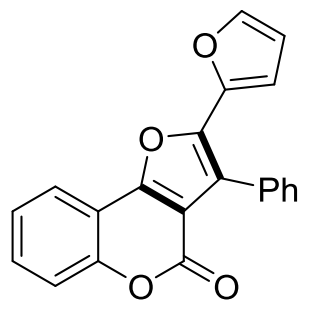

Following the TP-3, 6aj was obtained from 9a (75.1 mg, $0.3 \mathrm{mmol}), \mathrm{PPh}_{3}$ (86.6 mg, 1.1 equiv.), 2-furoyl chloride 3j (32.4 $\mu \mathrm{L}, 1.1$ equiv.) and DIPEA (78.4 $\mu \mathrm{L}, 1.5$ equiv.). The residue was purified by column chromatography $\left(\mathrm{SiO}_{2}\right.$, Hexanes/DCM=65:35) to give as a white solid (54.2mg, 55\% yield); $\mathrm{R}_{f}=0.49$ (DCM:Hex $\left.=2: 1\right) ; \mathrm{mp}=189.5-190.4{ }^{\circ} \mathrm{C}$.

${ }^{1} \mathbf{H}$ NMR $\left(400 \mathrm{MHz}, \mathrm{CDCl}_{3}, 25{ }^{\circ} \mathrm{C}\right) \delta / \mathrm{ppm}: 7.97(\mathrm{dd}, J=7.8,1.3 \mathrm{~Hz}, 1 \mathrm{H}), 7.57(\mathrm{dd}, J=7.8,1.7$ $\mathrm{Hz}, 2 \mathrm{H}), 7.51(\mathrm{td}, J=7.7,1.6 \mathrm{~Hz}, 1 \mathrm{H}), 7.49-7.40(\mathrm{~m}, 5 \mathrm{H}), 7.36(\mathrm{t}, J=7.8 \mathrm{~Hz}, 1 \mathrm{H}), 6.55(\mathrm{~d}, J=$ $3.5 \mathrm{~Hz}, 1 \mathrm{H}), 6.42(\mathrm{dd}, J=3.5,1.8 \mathrm{~Hz}, 1 \mathrm{H})$.

${ }^{13} \mathrm{C}$ NMR $\left(100 \mathrm{MHz} \mathrm{CDCl}_{3}, 25^{\circ} \mathrm{C}\right) \delta / \mathrm{ppm}: 157.2,156.5,152.7,144.3,143.7,143.2,130.8,130.2$, $129.1,128.5,128.1,124.4,120.9,120.5,117.1,112.5,111.5,110.8,109.6$.

IR $(\mathrm{KBr}) \tilde{v}\left(\mathrm{~cm}^{-1}\right): 2959,2360,1744,1458,1098,969,850,752,700$.

HRMS (ESI) $m / z:[\mathrm{M}+\mathrm{H}]^{+}$Calcd. for $\mathrm{C}_{21} \mathrm{H}_{13} \mathrm{O}_{4}$ 329.0814; found 329.0817 .

\section{2-Methyl-3-phenyl-4H-furo[3,2-c]chromen-4-one (6al)}

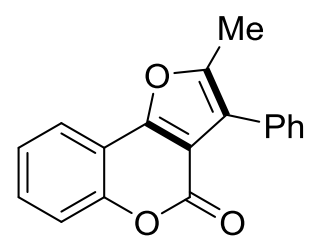


Following the TP-3, 6al was obtained from 9a $(75.1 \mathrm{mg}, 0.3 \mathrm{mmol}), \mathrm{PPh}_{3}(86.6 \mathrm{mg}, 1.1$ equiv.), acetyl chloride 31 (32.0 $\mu \mathrm{L}, 1.5$ equiv.) and DIPEA ( $78.4 \mu \mathrm{L}, 1.5$ equiv.). The residue was purified by column chromatography $\left(\mathrm{SiO}_{2}\right.$, Hexanes/DCM=65:35) to give as a white solid $(24.9 \mathrm{mg}, 30 \%$ yield); $\mathrm{R}_{f}=0.49$ (DCM:Hex $=2: 1$ ); $\mathrm{mp}=203.8-204.8^{\circ} \mathrm{C}$.

${ }^{1} \mathbf{H}$ NMR $\left(400 \mathrm{MHz}, \mathrm{CDCl}_{3}, 25^{\circ} \mathrm{C}\right) \delta / \mathrm{ppm}: 7.88(\mathrm{dd}, J=7.7,1.3 \mathrm{~Hz}, 1 \mathrm{H}), 7.54-7.46(\mathrm{~m}, 4 \mathrm{H})$, 7.44 (pt, $J=7.5 \mathrm{~Hz}, 2 \mathrm{H}), 7.38$ (t, $J=7.0 \mathrm{~Hz}, 1 \mathrm{H}), 7.34$ (t, $J=7.4 \mathrm{~Hz}, 1 \mathrm{H}), 2.53$ (s, 3H).

${ }^{13} \mathrm{C} \mathrm{NMR}\left(100 \mathrm{MHz}, \mathrm{CDCl}_{3}, 25^{\circ} \mathrm{C}\right) \delta / \mathrm{ppm}: 157.7,156.3,152.4,151.7,130.3,130.0,129.9,128.2$, 127.8, 124.3, 120.6, 120.5, 117.1, 112.8, 109.7, 12.6 .

IR (KBr) $\tilde{v}\left(\mathrm{~cm}^{-1}\right): 2968,2360,1743,1501,1084,961,852,750,698$.

HRMS (ESI) $m / z:[\mathrm{M}+\mathrm{H}]^{+}$Calcd. for $\mathrm{C}_{18} \mathrm{H}_{13} \mathrm{O}_{3} 277.0865$; found 277.0868 .

3-Phenyl-2-(trifluoromethyl)-4H-furo[3,2-c] chromen-4-one (6am)<smiles>O=c1oc2ccccc2c2oc(C(F)(F)F)c(-c3ccccc3)c12</smiles>

Following the TP-3, 6am was obtained from 9a (75.1 mg, $0.3 \mathrm{mmol}$ ), $\mathrm{PPh}_{3}(86.6 \mathrm{mg}, 1.1$ equiv.), trifluroacetic anhydride $3 \mathrm{~m}(62.6 \mu \mathrm{L}, 1.5$ equiv.) and DIPEA (78.4 $\mu \mathrm{L}, 1.5$ equiv.). The residue was purified by column chromatography $\left(\mathrm{SiO}_{2}\right.$, Hexanes/DCM= 70:30) to give as a white solid (39.6mg, 40\% yield); $\mathrm{R}_{f}=0.57$ (DCM:Hex $=2: 1$ ); $\mathrm{mp}=151.8-152.7^{\circ} \mathrm{C}$.

${ }^{1} \mathbf{H}$ NMR $\left(400 \mathrm{MHz}, \mathrm{CDCl}_{3}, 25{ }^{\circ} \mathrm{C}\right) \delta / \mathrm{ppm}: 7.99(\mathrm{dd}, J=7.8,1.5 \mathrm{~Hz}, 1 \mathrm{H}), 7.62(\mathrm{td}, J=7.7,1.6$ $\mathrm{Hz}, 1 \mathrm{H}), 7.55-7.46(\mathrm{~m}, 6 \mathrm{H}), 7.42(\mathrm{td}, J=7.5,1.3 \mathrm{~Hz}, 1 \mathrm{H})$.

${ }^{13} \mathrm{C} \mathrm{NMR}\left(100 \mathrm{MHz}, \mathrm{CDCl}_{3}, 25{ }^{\circ} \mathrm{C}\right) \delta / \mathrm{ppm}: 158.0,156.4,153.3,138.6\left(\mathrm{q},{ }^{2} J_{\mathrm{C}-\mathrm{F}}=41.0 \mathrm{~Hz}\right), 132.3$, $129.9,129.4,128.2,126.4,124.8,121.5,119.1\left(\mathrm{q},{ }^{1} J_{\mathrm{C}-\mathrm{F}}=270.0 \mathrm{~Hz}\right), 117.4,111.8,109.8$.

${ }^{19} \mathbf{F}$ NMR $\left(376 \mathrm{MHz}, \mathrm{CDCl}_{3}, 25{ }^{\circ} \mathrm{C}\right) \delta / \mathrm{ppm}:-60.39$

IR (KBr) $\tilde{v}\left(\mathrm{~cm}^{-1}\right): 2968,2362,1754,1425,1189,1121,972,753,720$.

HRMS (ESI) $m / z:[\mathrm{M}+\mathrm{H}]^{+}$Calcd. for $\mathrm{C}_{18} \mathrm{H}_{10} \mathrm{~F}_{3} \mathrm{O}_{3} 331.0582$; found 331.0587 .

\section{5-Benzoyl-2,3-diphenylfuro[3,2-c]quinolin-4(5H)-one (7aa)}

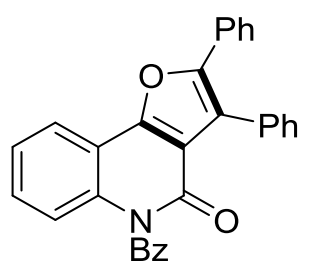

S35 
Following the TP-4, 7aa was obtained from $12 \mathbf{a}(106.0 \mathrm{mg}, 0.3 \mathrm{mmol}), \mathrm{PPh}_{3}(86.6 \mathrm{mg}, 1.1$ equiv.), benzoyl chloride 3a (38.3 $\mu \mathrm{L}, 1.1$ equiv.) and DIPEA (78.4 $\mu \mathrm{L}, 1.5$ equiv.). The residue was purified by column chromatography $\left(\mathrm{SiO}_{2}\right.$, Hexanes/EtOAc= 90:10) to give as a white solid (92.7mg, 70\% yield); $\mathrm{R}_{f}=0.48$ (EA:Hex $\left.=2: 8\right) ; \mathrm{mp}=227.1-228.0^{\circ} \mathrm{C}$.

${ }^{1} \mathbf{H}$ NMR $\left(400 \mathrm{MHz}, \mathrm{CDCl}_{3}, 25{ }^{\circ} \mathrm{C}\right) \delta / \mathrm{ppm}: 8.19(\mathrm{dd}, J=7.8,1.6 \mathrm{~Hz}, 1 \mathrm{H}), 7.90(\mathrm{dd}, J=8.0,1.6$ $\mathrm{Hz}, 2 \mathrm{H}), 7.64-7.57(\mathrm{~m}, 3 \mathrm{H}), 7.52(\mathrm{dd}, J=8.0,1.7 \mathrm{~Hz}, 2 \mathrm{H}), 7.44(\mathrm{pt}, J=8.0 \mathrm{~Hz}, 3 \mathrm{H}), 7.39-7.29$ $(\mathrm{m}, 7 \mathrm{H}), 7.06(\mathrm{~d}, J=8.1 \mathrm{~Hz}, 1 \mathrm{H})$.

${ }^{13} \mathrm{C} \mathrm{NMR}\left(100 \mathrm{MHz}, \mathrm{CDCl}_{3}, 25^{\circ} \mathrm{C}\right) \mathrm{\delta} / \mathrm{ppm}: 173.1,158.7,155.2,151.2,136.1,134.9,132.4,130.7$, $130.6,130.4,129.78,129.75,129.3,128.62,128.55,128.3,128.0,126.3,123.3,121.5,121.1$, $115.3,115.1,112.4$.

IR $(\mathrm{KBr}) \tilde{v}\left(\mathrm{~cm}^{-1}\right): 2923,2387,1723,1667,1232,1143,1013,856,750,698$.

HRMS (ESI) $m / z:[\mathrm{M}+\mathrm{H}]^{+}$Calcd. for $\mathrm{C}_{30} \mathrm{H}_{20} \mathrm{NO}_{3}$ 442.1443; found 442.1441 .

5-Benzoyl-3-phenyl-2-(trifluoromethyl)furo[3,2-c]quinolin-4(5H)-one(7am)

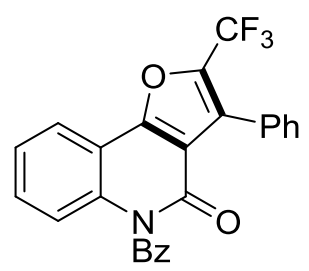

Following the TP-4, 7am was obtained from 12a (106.0 mg, $0.3 \mathrm{mmol}), \mathrm{PPh}_{3}(86.6 \mathrm{mg}, 1.1$ equiv.), trifluroacetic anhydride $3 \mathrm{~m}(62.6 \mu \mathrm{L}, 1.5$ equiv.) and DIPEA (78.4 $\mu \mathrm{L}, 1.5$ equiv.). The residue was purified by column chromatography $\left(\mathrm{SiO}_{2}, \mathrm{Hexanes} / \mathrm{EtOAc}=90: 10\right)$ to give as a white solid (52.0mg, $40 \%$ yield); $\mathrm{R}_{f}=0.55$ (EA:Hex $=2: 8$ ); $\mathrm{mp}=200.8-201.7^{\circ} \mathrm{C}$.

${ }^{1} \mathbf{H}$ NMR $\left(400 \mathrm{MHz}, \mathrm{CDCl}_{3}, 25{ }^{\circ} \mathrm{C}\right) \delta / \mathrm{ppm}: 8.18(\mathrm{dd}, J=8.1,1.3 \mathrm{~Hz}, 1 \mathrm{H}), 7.88(\mathrm{dd}, J=7.9,1.3$ $\mathrm{Hz}, 2 \mathrm{H}), 7.62(\mathrm{t}, J=7.5 \mathrm{~Hz}, 1 \mathrm{H}), 7.56-7.52(\mathrm{~m}, 2 \mathrm{H}), 7.49(\mathrm{td}, J=7.7,1.7 \mathrm{~Hz}, 1 \mathrm{H}), 7.44(\mathrm{t}, J=$ $7.8 \mathrm{~Hz}, 2 \mathrm{H}), 7.42-7.36(\mathrm{~m}, 4 \mathrm{H}), 7.09(\mathrm{~d}, J=8.3 \mathrm{~Hz}, 1 \mathrm{H})$.

${ }^{13} \mathbf{C ~ N M R}\left(100 \mathrm{MHz}, \mathrm{CDCl}_{3}, 25{ }^{\circ} \mathrm{C}\right) \delta / \mathrm{ppm}: 172.4,157.9,156.6,138.4\left(\mathrm{q},{ }^{2} J_{\mathrm{C}-\mathrm{F}}=40.9 \mathrm{~Hz}\right), 137.0$, $135.2,132.0,131.3,130.7,130.1,129.4,129.0,127.9,126.9,123.7,122.1,119.4\left(\mathrm{q},{ }^{1} J_{\mathrm{C}-\mathrm{F}}=270.2\right.$ $\mathrm{Hz}), 115.3,113.6,111.6$.

${ }^{19} \mathbf{F}$ NMR $\left(376 \mathrm{MHz}, \mathrm{CDCl}_{3}, 25{ }^{\circ} \mathrm{C}\right) \delta / \mathrm{ppm}:-60.64,-60.19$

IR (KBr) $\tilde{v}\left(\mathrm{~cm}^{-1}\right):$ 2989, 2356, 1732, 1673, 1450, 1232, 1139, 1014, 840, 751, 686.

HRMS (ESI) $m / z:[\mathrm{M}+\mathrm{H}]^{+}$Calcd. for $\mathrm{C}_{25} \mathrm{H}_{15} \mathrm{~F}_{3} \mathrm{NO}_{3}$ 434.1004; found 434.1007. 
General procedure for synthesis of Zwitterion: 2-oxo-3-((triphenylphosphonio)methyl)-2Hchromen-4-olate (2a)

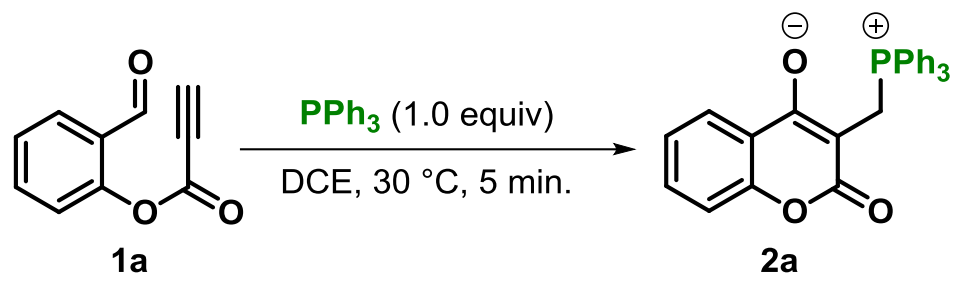

A dry and argon-flushed $10 \mathrm{~mL}$ Schlenk flask equipped with a magnetic stir bar and septum was sequentially charged with $1 \mathrm{a}(87.08 \mathrm{mg}, 0.5 \mathrm{mmol}), \mathrm{PPh}_{3}(131.14 \mathrm{mg}, 1.0$ equiv) and anhydrous DCE $(5 \mathrm{~mL})$. The reaction mixture was stirred for $5 \mathrm{~min}$ at $30^{\circ} \mathrm{C}$ and the residue was precipitated out in $5 \mathrm{~min}$. After completion of the reaction, solvent was removed in vacuo and the crude residue was washed with hexanes to get pure compound 2a as gray solid in $98 \%$ yield (213.9 $\mathrm{mg}$ ).

$\mathrm{R}_{f}=0.66($ EA:Hex $=2: 8) ; \mathrm{mp}=195.1-196.0^{\circ} \mathrm{C}$.

${ }^{1} \mathbf{H}$ NMR (400 MHz, $\mathrm{CDCl}_{3}, 25^{\circ} \mathrm{C}$ ) $\delta / \mathrm{ppm}: 7.87(\mathrm{dd}, J=7.7,1.7 \mathrm{~Hz}, 1 \mathrm{H}), 7.79(\mathrm{dd}, J=8.0,1.3$ $\mathrm{Hz}, 3 \mathrm{H}), 7.76$ (dd, $J=7.9,1.4 \mathrm{~Hz}, 3 \mathrm{H}), 7.67(\mathrm{td}, J=7.5,1.7 \mathrm{~Hz}, 3 \mathrm{H}), 7.54(\mathrm{td}, J=7.6,3.4 \mathrm{~Hz}$, $6 \mathrm{H}), 7.33(\mathrm{td}, J=7.4,1.6 \mathrm{~Hz}, 1 \mathrm{H}), 7.15-7.06(\mathrm{~m}, 2 \mathrm{H}), 4.50(\mathrm{~d}, J=9.9 \mathrm{~Hz}, 2 \mathrm{H})$.

${ }^{13} \mathrm{C}$ NMR $\left(100 \mathrm{MHz}, \mathrm{CDCl}_{3}, 25^{\circ} \mathrm{C}\right) \delta / \mathrm{ppm}: 174.9,165.7\left(\mathrm{~d},{ }^{3} J_{\mathrm{C}-\mathrm{P}}=2.6 \mathrm{~Hz}\right), 153.9,134.0,134.05$ $(\mathrm{d}, J=12.5 \mathrm{~Hz}), 130.3,129.4$ (d, $J=12.6 \mathrm{~Hz}), 124.7,122.2,122.1,121.1,120.3,116.2,86.15$ (d, $\left.{ }^{2} J_{\mathrm{C}-\mathrm{P}}=6.9 \mathrm{~Hz}\right), 24.4\left(\mathrm{~d},{ }^{1} J_{\mathrm{C}-\mathrm{P}}=46.0 \mathrm{~Hz}\right)$,

${ }^{31} \mathbf{P}$ NMR (162 MHz, $\left.\mathrm{CDCl}_{3}, 25^{\circ} \mathrm{C}\right) \delta / \mathrm{ppm:} 23.76$.

IR (KBr) $\tilde{v}\left(\mathrm{~cm}^{-1}\right): 3425,2991,1648,1601,1534,1438,1110,1043,850,751,689$.

HRMS (ESI) $m / z$ : $[\mathrm{M}+\mathrm{H}]^{+}$Calcd. for $\mathrm{C}_{28} \mathrm{H}_{22} \mathrm{O}_{3} \mathrm{P}$ 437.1307; found 437.1308.

General procedure for synthesis of perfluorophenyl ylide (11an)<smiles>C#CC(=O)Oc1ccccc1C=O</smiles> 
A dry and argon-flushed $10 \mathrm{~mL}$ Schlenk flask equipped with a magnetic stir bar and septum was sequentially charged with $1 \mathrm{a}$ (52.24 mg, $0.3 \mathrm{mmol}), \mathrm{PPh}_{3}$ ( $86.6 \mathrm{mg}, 1.1$ equiv), anhydrous DCE (3 mL), 2,3,4,5,6-pentafluorobenzoyl chloride 3n (95.1 $\mu \mathrm{L}, 2.2$ equiv.) and DIPEA (78.4 $\mu \mathrm{L}, 1.5$ equiv). The reaction mixture was stirred for $10 \mathrm{~h}$ at $60^{\circ} \mathrm{C}$. After completion of the reaction, solvent was removed in vacuo and the residue was purified by silica gel column chromatography (eluent: ethyl acetate/n-hexanes: $15 / 85$ ) to give the product 11an as yellow solid (131.1 mg, 53\% yield). $\mathrm{R}_{f}=0.16($ EA:Hex $=2: 8) ; \mathrm{mp}=194.8-195.7^{\circ} \mathrm{C}$.

${ }^{1} \mathbf{H}$ NMR $\left(400 \mathrm{MHz}, \mathrm{CDCl}_{3}, 25{ }^{\circ} \mathrm{C}\right) \delta / \mathrm{ppm}: 8.18(\mathrm{dd}, J=7.5 \mathrm{~Hz}, 2 \mathrm{H}), 7.81(\mathrm{pt}, J=7.5 \mathrm{~Hz}, 2 \mathrm{H})$, $7.65-7.53(\mathrm{~m}, 4 \mathrm{H}), 7.51-7.28(\mathrm{~m}, 8 \mathrm{H}), 7.24-7.15(\mathrm{~m}, 3 \mathrm{H})$.

${ }^{13} \mathrm{C}$ NMR $\left(100 \mathrm{MHz}, \mathrm{CDCl}_{3}, 25{ }^{\circ} \mathrm{C}\right) \delta / \mathrm{ppm}: 173.5,173.4,162.1,155.9(\mathrm{~d}, J=6.0 \mathrm{~Hz}), 155.3$, $151.9,146.8\left(\mathrm{~m},{ }^{1} J_{\mathrm{C}-\mathrm{F}}=264.0 \mathrm{~Hz}\right), 144.7\left(\mathrm{~m},{ }^{1} J_{\mathrm{C}-\mathrm{F}}=265.3 \mathrm{~Hz}\right), 140.6\left(\mathrm{~m},{ }^{1} J_{\mathrm{C}-\mathrm{F}}=250.6 \mathrm{~Hz}\right), 137.9$ $\left(\mathrm{m},{ }^{1} J_{\mathrm{C}-\mathrm{F}}=256.6 \mathrm{~Hz}\right), 137.0\left(\mathrm{~m},{ }^{1} J_{\mathrm{C}-\mathrm{F}}=254.3 \mathrm{~Hz}\right), 134.1(\mathrm{~d}, J=9.4 \mathrm{~Hz}), 133.7(\mathrm{t}, J=9.8 \mathrm{~Hz})$, 132.4, 131.9, $128.9(\mathrm{~d}, J=13.7 \mathrm{~Hz}), 128.6(\mathrm{~m}, J=6.7 \mathrm{~Hz}), 126.6(\mathrm{~d}, J=92.3 \mathrm{~Hz}), 124.8(\mathrm{~d}, J=$ $88.0 \mathrm{~Hz}), 122.7(\mathrm{~d}, J=95.3 \mathrm{~Hz}), 122.2,118.3(\mathrm{~d}, J=9.4 \mathrm{~Hz}), 117.4\left(\mathrm{~m},{ }^{2} J_{\mathrm{C}-\mathrm{F}}=22.6 \mathrm{~Hz}\right), 116.8$, 115.2, $105.2\left(\mathrm{pt},{ }^{2} J_{\mathrm{C}-\mathrm{F}}=12.7 \mathrm{~Hz}\right), 66.6(\mathrm{~d}, J=115.2 \mathrm{~Hz})$.

${ }^{19}$ F NMR $\left(376 \mathrm{MHz}, \mathrm{CDCl}_{3}, 25^{\circ} \mathrm{C}\right) \delta / \mathrm{ppm}:-134.7(\mathrm{~d}, J=16.4 \mathrm{~Hz}),-141.4,-143.2,-143.5,-155.6$ (t, $J=21.0 \mathrm{~Hz}$ ), -159.4 (pt, $J=18.6 \mathrm{~Hz}$ ), $-161.9,-162.8$.

${ }^{31} \mathbf{P}$ NMR (162 MHz, $\left.\mathrm{CDCl}_{3}, 25^{\circ} \mathrm{C}\right) \delta / \mathrm{ppm:} 18.96$.

IR $(\mathrm{KBr}) \tilde{v}\left(\mathrm{~cm}^{-1}\right): 3423,1734,1644,1518,1322,1188,1101,987,923,750,513$.

HRMS (ESI) $m / z:[\mathrm{M}+\mathrm{H}]^{+}$Calcd. for $\mathrm{C}_{42} \mathrm{H}_{20} \mathrm{~F}_{10} \mathrm{O}_{5} \mathrm{P}$ 825.0889; found 825.0884.

\section{References}

(1) Deng, Z.-X.; Zheng, Y.; Xie, Z.-Z.; Gao, Y.-H.; Xiao, J.-A.; Xie, S.-Q.; Xiang, H.-Y.; Chen, X.-Q.; Yang, H., Phosphine-Mediated MBH-Type/Umpolung Addition Domino Sequence: Divergent Construction of Coumarins. Org. Lett. 2020, 22, 488-492.

(2) Zhang, J.; Han, X.; Lu, X., Synthesis of 2-Quinolinones through Palladium(II) Acetate Catalyzed Cyclization of $N$-(2-Formylaryl)alkynamides. Synlett 2015, 26, 1744-1748. 


\section{X-Ray Crystallographic Data for Selected Compounds:}

a) 4aa (CCDC no. 2039605): The thermal ellipsoid drawn at $60 \%$ probability level.

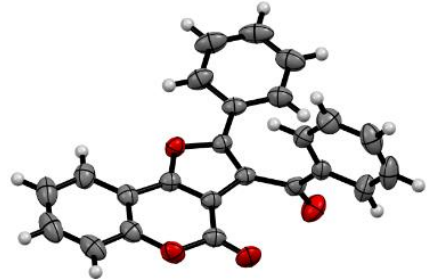

Empirical formula

Formula weight

Temperature

Wavelength

Crystal system

Space group

Unit cell dimensions

Volume

$\mathrm{Z}$

Density (calculated)

Absorption coefficient

$\mathrm{F}(000)$

Crystal size

Theta range for data collection

Index ranges

Reflections collected

Independent reflections

Completeness to theta $=25.19^{\circ}$

Absorption correction

Max. and min. transmission

Refinement method

Data / restraints / parameters

Goodness-of-fit on $\mathrm{F}^{2}$

Final $\mathrm{R}$ indices [I>2sigma(I)]

$\mathrm{R}$ indices (all data)

Largest diff. peak and hole
The purified compound 4aa is dissolved in a mixed solvent of $\mathrm{CH}_{2} \mathrm{Cl}_{2}$ and hexanes to slowly evaporate. After few days, colorless crystals were obtained.

C24 H14 O4

366.35

200(2) K

$0.71073 \AA$

Triclinic

$\mathrm{P}-1$

$\mathrm{a}=11.231(4) \AA \quad \alpha=96.161(14)^{\circ}$.

$\mathrm{b}=11.272(4) \AA \quad \beta=97.183(14)^{\circ}$.

$\mathrm{c}=14.972(5) \AA \quad \gamma=106.841(15)^{\circ}$.

$1779.2(10) \AA^{3}$

4

$1.368 \mathrm{Mg} / \mathrm{m}^{3}$

$0.093 \mathrm{~mm}^{-1}$

760

$0.72 \times 0.63 \times 0.18 \mathrm{~mm}^{3}$

2.18 to $25.19^{\circ}$.

$-13<=\mathrm{h}<=13,-13<=\mathrm{k}<=13,-17<=1<=17$

51877

$6268[\mathrm{R}(\mathrm{int})=0.0512]$

$97.8 \%$

multi-scan

0.9834 and 0.9359

Full-matrix least-squares on $\mathrm{F}^{2}$

$6268 / 0 / 505$

1.047

$\mathrm{R} 1=0.0343, \mathrm{wR} 2=0.0833$

$\mathrm{R} 1=0.0413, \mathrm{wR} 2=0.0881$

0.189 and -0.164 e. $\AA^{-3}$ 
b) 4 ba (CCDC no. 2039745): The thermal ellipsoid drawn at 55\% probability level.

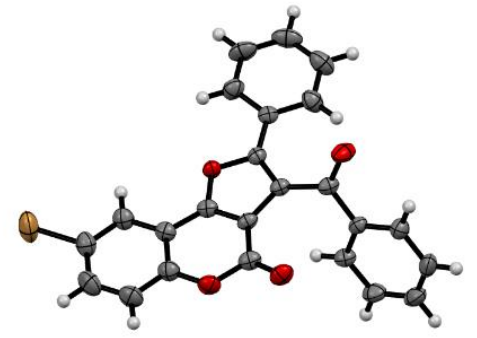

Empirical formula

Formula weight

Temperature

Wavelength

Crystal system

Space group

Unit cell dimensions

Volume

$\mathrm{Z}$

Density (calculated)

Absorption coefficient

$\mathrm{F}(000)$

Crystal size

Theta range for data collection

Index ranges

Reflections collected

Independent reflections

Completeness to theta $=25.01^{\circ}$

Absorption correction

Max. and min. transmission

Refinement method

Data / restraints / parameters

Goodness-of-fit on $\mathrm{F}^{2}$

Final $\mathrm{R}$ indices [I $>2 \operatorname{sigma}(\mathrm{I})]$

$\mathrm{R}$ indices (all data)

Largest diff. peak and hole
The purified compound $\mathbf{4 b a}$ is dissolved in a mixed solvent of $\mathrm{CH}_{2} \mathrm{Cl}_{2}$ and hexanes to slowly evaporate. After few days, colorless crystals were obtained.
C24 H13 Br O4

445.25

200(2) K

$0.71073 \AA$

Monoclinic

P 21/c

$\mathrm{a}=7.9218(5) \AA$

$\alpha=90^{\circ}$.

$\mathrm{b}=24.9409(15) \AA$

$\beta=102.184(2)^{\circ}$.

$\mathrm{c}=9.8753(7) \AA$ $\gamma=90^{\circ}$.

$1907.2(2) \AA^{3}$

4

$1.551 \mathrm{Mg} / \mathrm{m}^{3}$

$2.184 \mathrm{~mm}^{-1}$

896

$0.40 \times 0.03 \times 0.02 \mathrm{~mm}^{3}$

2.26 to $25.01^{\circ}$.

$-9<=\mathrm{h}<=9,-29<=\mathrm{k}<=29,-11<=\mathrm{l}<=11$

25523

$3356[\mathrm{R}(\mathrm{int})=0.0624]$

$99.7 \%$

multi-scan

0.9576 and 0.4753

Full-matrix least-squares on $\mathrm{F}^{2}$

$3356 / 0 / 262$

1.013

$\mathrm{R} 1=0.0398, \mathrm{wR} 2=0.0876$

$\mathrm{R} 1=0.0670, \mathrm{wR} 2=0.1012$

0.513 and -0.612 e. $\AA^{-3}$ 
c) 4ah (CCDC no. 2039742): The thermal ellipsoid drawn at 60\% probability level.

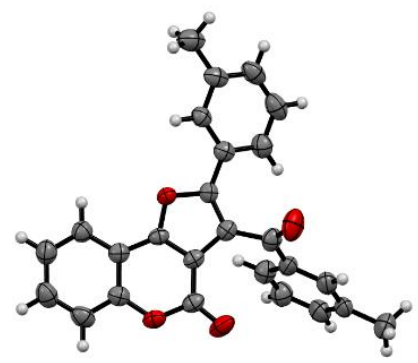

Empirical formula

Formula weight

Temperature

Wavelength

Crystal system

Space group

Unit cell dimensions

Volume

Z

Density (calculated)

Absorption coefficient

$\mathrm{F}(000)$

Crystal size

Theta range for data collection

Index ranges

Reflections collected

Independent reflections

Completeness to theta $=25.05^{\circ}$

Absorption correction

Max. and min. transmission

Refinement method

Data / restraints / parameters

Goodness-of-fit on $\mathrm{F}^{2}$

Final R indices [I $>2 \operatorname{sigma(I)]~}$

$\mathrm{R}$ indices (all data)

Largest diff. peak and hole
The purified compound $\mathbf{4 a h}$ is dissolved in a mixed solvent of $\mathrm{CH}_{2} \mathrm{Cl}_{2}$ and hexanes to slowly evaporate. After few days, colorless crystals were obtained.
C26 H18 O4

394.40

200(2) K

$0.71073 \AA$

Monoclinic

P 21/c

$\mathrm{a}=7.5088(7) \AA \quad \alpha=90^{\circ}$.

$\mathrm{b}=21.488(2) \AA \quad \beta=105.125(3)^{\circ}$.

$\mathrm{c}=12.3407(13) \AA \quad \gamma=90^{\circ}$.

1922.2(3) $\AA^{3}$

4

$1.363 \mathrm{Mg} / \mathrm{m}^{3}$

$0.092 \mathrm{~mm}^{-1}$

824

$0.18 \times 0.16 \times 0.05 \mathrm{~mm}^{3}$

1.95 to $25.05^{\circ}$.

$-8<=\mathrm{h}<=8,-25<=\mathrm{k}<=25,-14<=1<=14$

28261

$3383[\mathrm{R}(\mathrm{int})=0.0627]$

$99.9 \%$

multi-scan

0.9954 and 0.9837

Full-matrix least-squares on $\mathrm{F}^{2}$

3383 / 0 / 273

1.076

$\mathrm{R} 1=0.0770, \mathrm{wR} 2=0.2016$

$\mathrm{R} 1=0.0924, \mathrm{wR} 2=0.2125$

0.767 and -0.293 e. $\AA^{-3}$ 
d) 8 (CCDC no. 2039756): The thermal ellipsoid drawn at 60\% probability level.

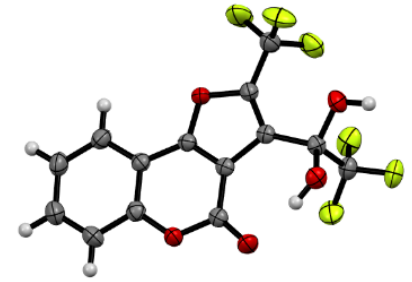

Empirical formula

Formula weight

Temperature

Wavelength

Crystal system

Space group

Unit cell dimensions

Volume

$\mathrm{Z}$

Density (calculated)

Absorption coefficient

$\mathrm{F}(000)$

Crystal size

Theta range for data collection

Index ranges

Reflections collected

Independent reflections

Completeness to theta $=24.97^{\circ}$

Absorption correction

Max. and min. transmission

Refinement method

Data / restraints / parameters

Goodness-of-fit on $\mathrm{F}^{2}$

Final R indices [I $>2 \operatorname{sigma}(\mathrm{I})]$

$\mathrm{R}$ indices (all data)

Largest diff. peak and hole
The purified compound $\mathbf{8}$ is dissolved in a mixed solvent of $\mathrm{CH}_{2} \mathrm{Cl}_{2}$ and hexanes to slowly evaporate. After few days, colorless crystals were obtained.
C14 H6 F6 N0 O5

368.19

200(2) K

$0.71073 \AA$

Monoclinic

$\mathrm{C} 2 / \mathrm{c}$

$\mathrm{a}=33.0758(18) \AA$ $\alpha=90^{\circ}$.

$\mathrm{b}=5.0398(3) \AA$ $\beta=90.357(2)^{\circ}$.

$\mathrm{c}=15.6721(10) \AA$ $\gamma=90^{\circ}$.

2612.4(3) $\AA^{3}$

8

$1.872 \mathrm{Mg} / \mathrm{m}^{3}$

$0.195 \mathrm{~mm}^{-1}$

1472

$0.56 \times 0.04 \times 0.02 \mathrm{~mm}^{3}$

2.60 to $24.97^{\circ}$.

$-35<=\mathrm{h}<=38,-5<=\mathrm{k}<=5,-18<=\mathrm{l}<=18$

19432

$2269[\mathrm{R}(\mathrm{int})=0.0773]$

$99.6 \%$

multi-scan

0.9961 and 0.8987

Full-matrix least-squares on $\mathrm{F}^{2}$

2269 / 0 / 226

1.058

$\mathrm{R} 1=0.0399, \mathrm{wR} 2=0.0931$

$\mathrm{R} 1=0.0598, \mathrm{wR} 2=0.1096$

0.246 and -0.311 e. $\AA^{-3}$ 
e) 6ba (CCDC no. 2039752): The thermal ellipsoid drawn at 60\% probability level.

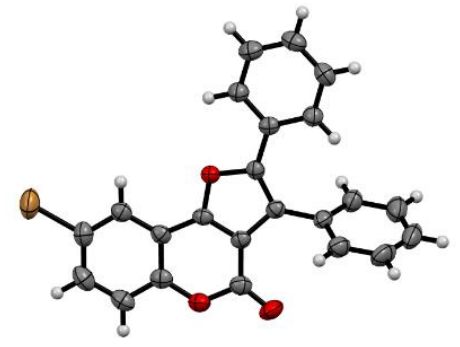

Empirical formula

Formula weight

Temperature

Wavelength

Crystal system

Space group

Unit cell dimensions

Volume

$\mathrm{Z}$

Density (calculated)

Absorption coefficient

$\mathrm{F}(000)$

Crystal size

Theta range for data collection

Index ranges

Reflections collected

Independent reflections

Completeness to theta $=25.06^{\circ}$

Absorption correction

Max. and min. transmission

Refinement method

Data / restraints / parameters

Goodness-of-fit on $\mathrm{F}^{2}$

Final R indices [I>2sigma(I)]

$\mathrm{R}$ indices (all data)

Largest diff. peak and hole
The purified compound $\mathbf{6 b a}$ is dissolved in a mixed solvent of $\mathrm{CH}_{2} \mathrm{Cl}_{2}$ and hexanes to slowly evaporate. After few days, colorless crystals were obtained.
C23 H13 Br O3

417.24

200(2) K

$0.71073 \AA$

Monoclinic

P 21/n

$\mathrm{a}=12.0278(6) \AA$ $\alpha=90^{\circ}$.

$\mathrm{b}=6.9779(3) \AA$ $\beta=102.7790(10)^{\circ}$.

$\mathrm{c}=21.5149(10) \AA$ $\gamma=90^{\circ}$.

$1760.99(14) \AA^{3}$

4

$1.574 \mathrm{Mg} / \mathrm{m}^{3}$

$2.356 \mathrm{~mm}^{-1}$

840

$0.68 \times 0.07 \times 0.03 \mathrm{~mm}^{3}$

2.17 to $25.06^{\circ}$.

$-14<=\mathrm{h}<=14,-8<=\mathrm{k}<=8,-25<=1<=25$

32319

$3114[\mathrm{R}(\mathrm{int})=0.0520]$

$99.8 \%$

multi-scan

0.9327 and 0.2973

Full-matrix least-squares on $\mathrm{F}^{2}$

3114 / 0 / 244

1.024

$\mathrm{R} 1=0.0335, \mathrm{wR} 2=0.0870$

$\mathrm{R} 1=0.0472, w \mathrm{R} 2=0.0949$

0.319 and -0.498 e. $\AA^{-3}$ 
f) 6ae (CCDC no. 2039765): The thermal ellipsoid drawn at 60\% probability level.

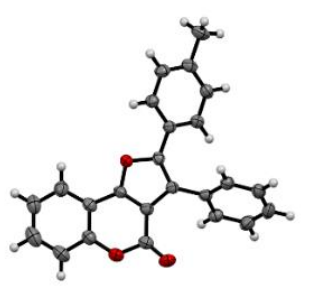

Empirical formula

Formula weight

Temperature

Wavelength

Crystal system

Space group

Unit cell dimensions

Volume

Z

Density (calculated)

Absorption coefficient

$\mathrm{F}(000)$

Crystal size

Theta range for data collection

Index ranges

Reflections collected

Independent reflections

Completeness to theta $=25.07^{\circ}$

Absorption correction

Max. and min. transmission

Refinement method

Data / restraints / parameters

Goodness-of-fit on $\mathrm{F}^{2}$

Final R indices [I $>2 \operatorname{sigma}(\mathrm{I})]$

$\mathrm{R}$ indices (all data)

Absolute structure parameter

Largest diff. peak and hole
The purified compound 6ae is dissolved in a mixed solvent of $\mathrm{CH}_{2} \mathrm{Cl}_{2}$ and hexanes to slowly evaporate. After few days, colorless crystals were obtained.

\section{C24 H16 O3}

352.37

200(2) K

$0.71073 \AA$

Tetragonal

I -4

$\mathrm{a}=24.6105(8) \AA \quad \alpha=90^{\circ}$.

$\mathrm{b}=24.6105(8) \AA \quad \beta=90^{\circ}$.

$\mathrm{c}=6.1689(3) \AA \quad \gamma=90^{\circ}$.

3736.4(3) $\AA^{3}$

8

$1.253 \mathrm{Mg} / \mathrm{m}^{3}$

$0.082 \mathrm{~mm}^{-1}$

1472

$0.79 \times 0.07 \times 0.03 \mathrm{~mm}^{3}$

2.62 to $25.07^{\circ}$.

$-29<=\mathrm{h}<=29,-29<=\mathrm{k}<=29,-6<=\mathrm{l}<=7$

18727

$3305[\mathrm{R}(\mathrm{int})=0.0583]$

$99.6 \%$

multi-scan

0.9975 and 0.9380

Full-matrix least-squares on $\mathrm{F}^{2}$

3305 / 0 / 245

1.034

$\mathrm{R} 1=0.0440, \mathrm{wR} 2=0.1193$

$\mathrm{R} 1=0.0476, \mathrm{wR} 2=0.1227$

$0.1(14)$

0.319 and -0.190 e. $\AA^{-3}$ 
g) 2a (CCDC no. 2039766): The thermal ellipsoid drawn at 60\% probability level.

(*A PLATON_SQUEEZE procedure was used to deal with the disorder of half solvation of $\mathrm{CH}_{2} \mathrm{Cl}_{2}$ )

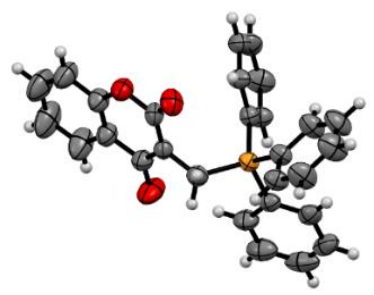

Empirical formula

Formula weight

Temperature

Wavelength

Crystal system

Space group

Unit cell dimensions

Volume

Z

Density (calculated)

Absorption coefficient

$\mathrm{F}(000)$

Crystal size

Theta range for data collection

Index ranges

Reflections collected

Independent reflections

Completeness to theta $=25.06^{\circ}$

Absorption correction

Max. and min. transmission

Refinement method

Data / restraints / parameters

Goodness-of-fit on $\mathrm{F}^{2}$

Final $\mathrm{R}$ indices [I $>2 \operatorname{sigma}(\mathrm{I})]$

$\mathrm{R}$ indices (all data)

Largest diff. peak and hole
The purified compound $\mathbf{2 a}$ is dissolved in a mixed solvent of $\mathrm{CH}_{2} \mathrm{Cl}_{2}$ and hexanes to slowly evaporate. After few days, colorless crystals were obtained.
C28 H21 O3 P

436.42

200(2) K

$0.71073 \AA$

Monoclinic

P 21/n

$\mathrm{a}=16.632(2) \AA$

$\alpha=90^{\circ}$.

$\mathrm{b}=8.7182(10) \AA$

$\beta=99.362(3)^{\circ}$.

$\mathrm{c}=17.510(2) \AA$ $\gamma=90^{\circ}$.

2505.3(5) $\AA^{3}$

4

$1.157 \mathrm{Mg} / \mathrm{m}^{3}$

$0.135 \mathrm{~mm}^{-1}$

912

$0.35 \times 0.06 \times 0.01 \mathrm{~mm}^{3}$

2.62 to $25.06^{\circ}$.

$-19<=\mathrm{h}<=19,-10<=\mathrm{k}<=10,-20<=\mathrm{l}<=20$

41773

$4435[\mathrm{R}(\mathrm{int})=0.1320]$

$99.7 \%$

multi-scan

0.9987 and 0.9544

Full-matrix least-squares on $\mathrm{F}^{2}$

4435 / 0 / 289

1.076

$\mathrm{R} 1=0.0720, \mathrm{wR} 2=0.1505$

$\mathrm{R} 1=0.1318, \mathrm{wR} 2=0.1741$

0.199 and -0.316 e. $\AA^{-3}$ 
h) 11an (CCDC no. 2039754): The thermal ellipsoid drawn at 50\% probability level.

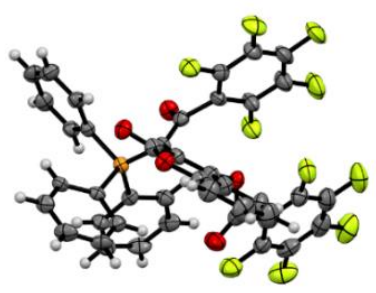

Empirical formula

Formula weight

Temperature

Wavelength

Crystal system

Space group

Unit cell dimensions

Volume

$\mathrm{Z}$

Density (calculated)

Absorption coefficient

$\mathrm{F}(000)$

Crystal size

Theta range for data collection

Index ranges

Reflections collected

Independent reflections

Completeness to theta $=25.03^{\circ}$

Absorption correction

Max. and min. transmission

Refinement method

Data / restraints / parameters

Goodness-of-fit on $\mathrm{F}^{2}$

Final $\mathrm{R}$ indices [I>2sigma(I)]

$\mathrm{R}$ indices (all data)

Absolute structure parameter

Largest diff. peak and hole
The purified compound 11an is dissolved in a mixed solvent of $\mathrm{CH}_{2} \mathrm{Cl}_{2}$ and hexanes to slowly evaporate. After few days, colorless crystals were obtained.
C42 H19 F10 O5 P

824.54

200(2) K

$0.71073 \AA$

Orthorhombic

P n a 21

$\mathrm{a}=21.5688(6) \AA \quad \alpha=90^{\circ}$.

$\mathrm{b}=18.5961(6) \AA \quad \beta=90^{\circ}$.

$\mathrm{c}=17.9622(6) \AA \quad \gamma=90^{\circ}$.

7204.6(4) $\AA^{3}$

8

$1.520 \mathrm{Mg} / \mathrm{m}^{3}$

$0.176 \mathrm{~mm}^{-1}$

3328

$0.12 \times 0.11 \times 0.04 \mathrm{~mm}^{3}$

2.18 to $25.03^{\circ}$.

$-25<=\mathrm{h}<=25,-14<=\mathrm{k}<=22,-21<=1<=21$

42096

$12610[\mathrm{R}(\mathrm{int})=0.0659]$

$99.8 \%$

multi-scan

0.9930 and 0.9792

Full-matrix least-squares on $\mathrm{F}^{2}$

12610 / 1 / 1045

0.922

$\mathrm{R} 1=0.0474, \mathrm{wR} 2=0.0957$

$\mathrm{R} 1=0.0826, \mathrm{wR} 2=0.1107$

$0.12(9)$

0.301 and -0.234 e. $\AA^{-3}$ 
VIII. ${ }^{1} \mathrm{H}$ NMR, ${ }^{13} \mathrm{C}$ NMR, ${ }^{31} \mathrm{P}$ NMR and ${ }^{19} \mathrm{~F}$ NMR Spectra of All New Compounds

$\underline{{ }^{1} \mathrm{H} \text { NMR Spectrum of } \mathbf{1 a}\left(\mathrm{CDCl}_{3}, 400 \mathrm{MHz}\right)}$

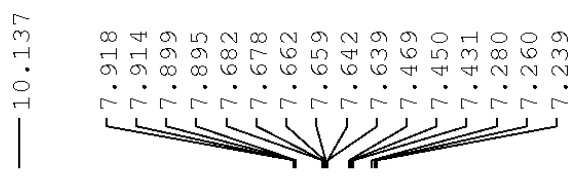

F2 - Acquisition Parameters

Date__ 20200820

Time 10.57

INSTRUM spect

$\begin{array}{ll}\text { PROBHD } & 5 \mathrm{~mm} \\ \text { PULPROG } & \end{array}$

2930
32768

SOLVENT CDCl3

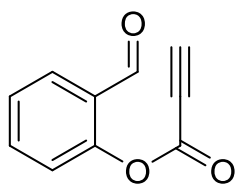

DS

SWH $\quad 7211.539 \mathrm{~Hz}$

AQ $\quad 2.2719147 \mathrm{sec}$

RG

DW

$\mathrm{DE}$

$1 \mathrm{a}$

69.333 usec

69.333 usec
10.06 usec

$\mathrm{TE}$

D1

$=====$ CHAHTTE $\mathrm{f} 1$

SFO1

SFO1 $400.1324008 \mathrm{MH}$

N1 $1 \mathrm{H}$

$\begin{array}{lr}\text { P1 } & 15.00 \text { usec } \\ \text { PLW1 } & 11.39999962 \mathrm{~W}\end{array}$

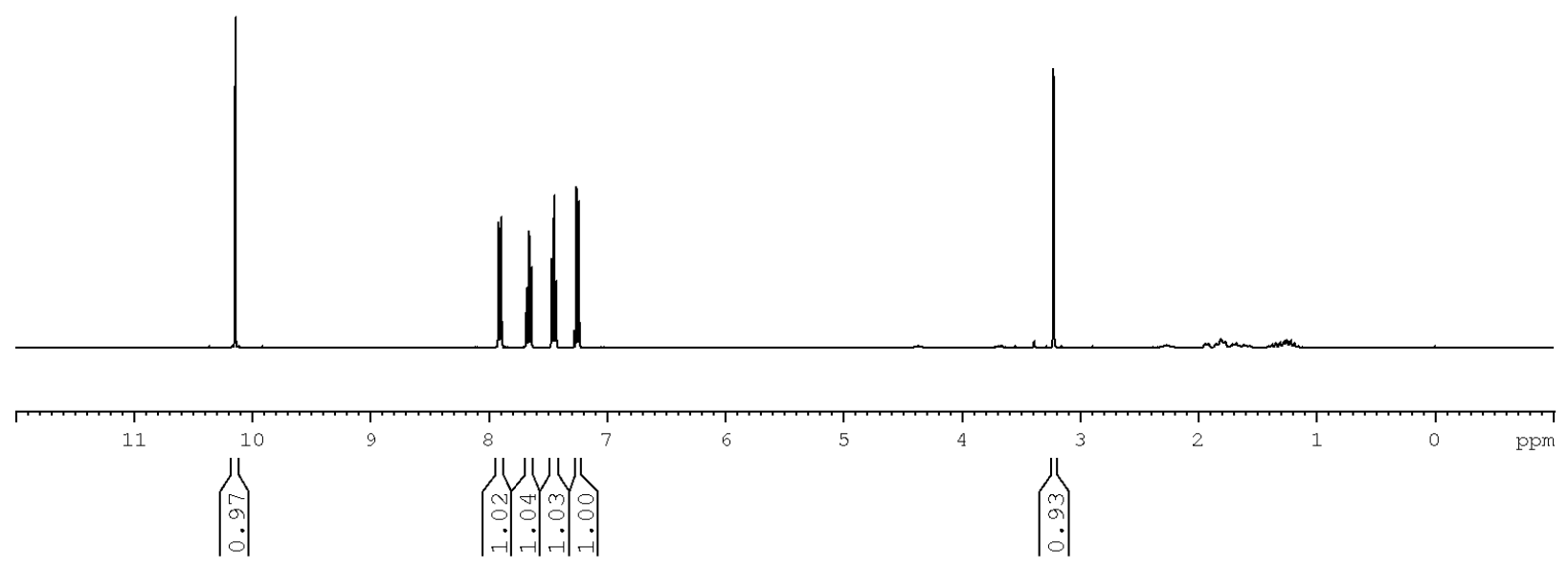

2 - Processing parameters

I 16384

SF $\quad 400.1300013 \mathrm{MHz}$

WDW

SSB

LB

$\mathrm{PC}$

EM 
${ }^{13} \mathrm{C}$ NMR Spectrum of $1 \mathrm{a}\left(\mathrm{CDCl}_{3}, 100 \mathrm{MHz}\right)$
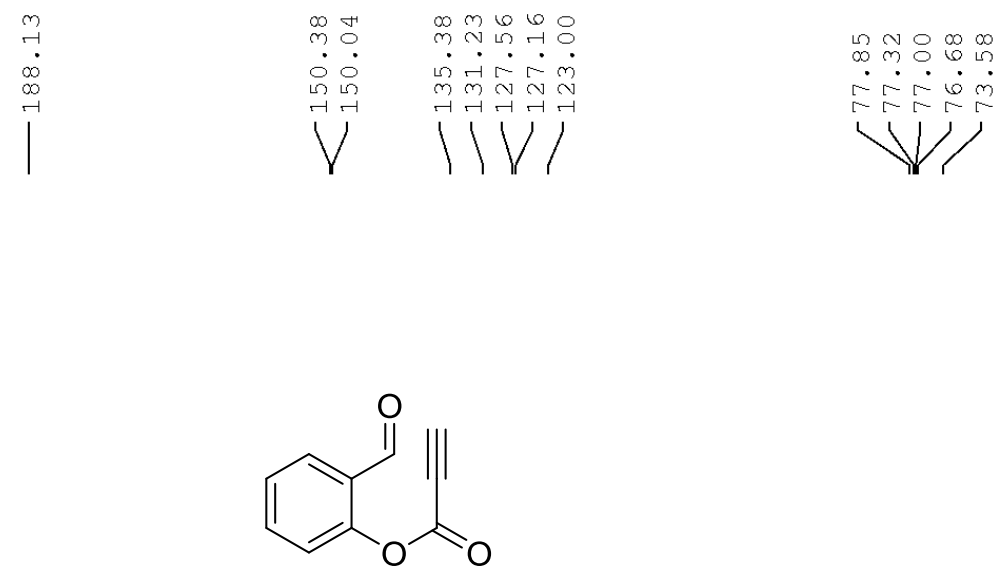

$1 \mathbf{a}$
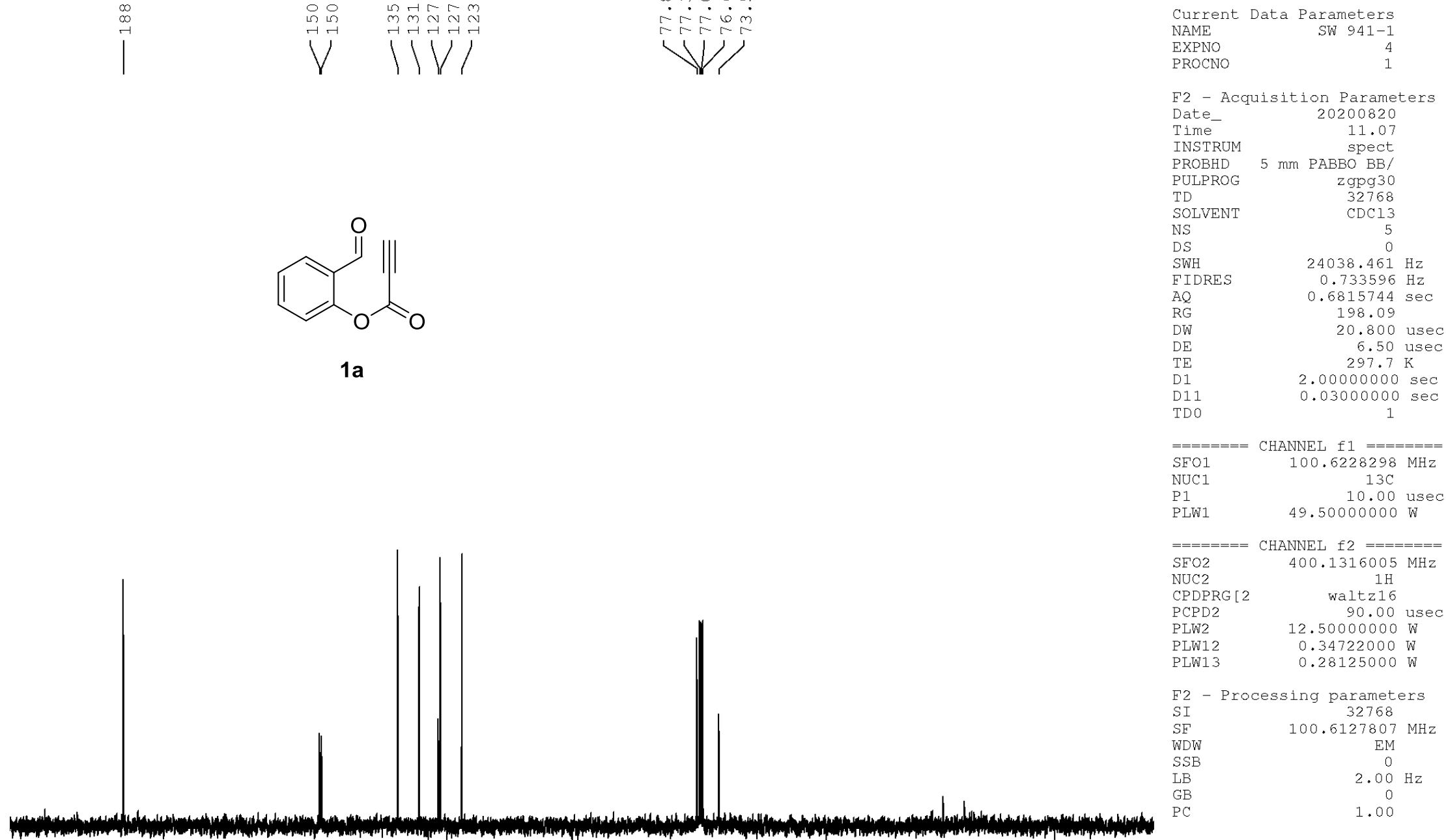

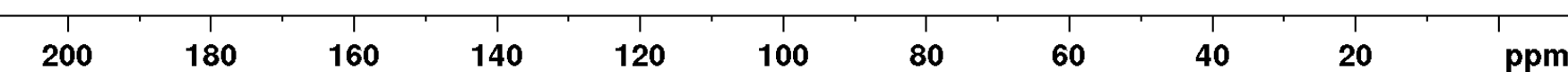


${ }^{1} \mathrm{H}$ NMR Spectrum of $\mathbf{1 b}\left(\mathrm{CDCl}_{3}, 400 \mathrm{MHz}\right)$

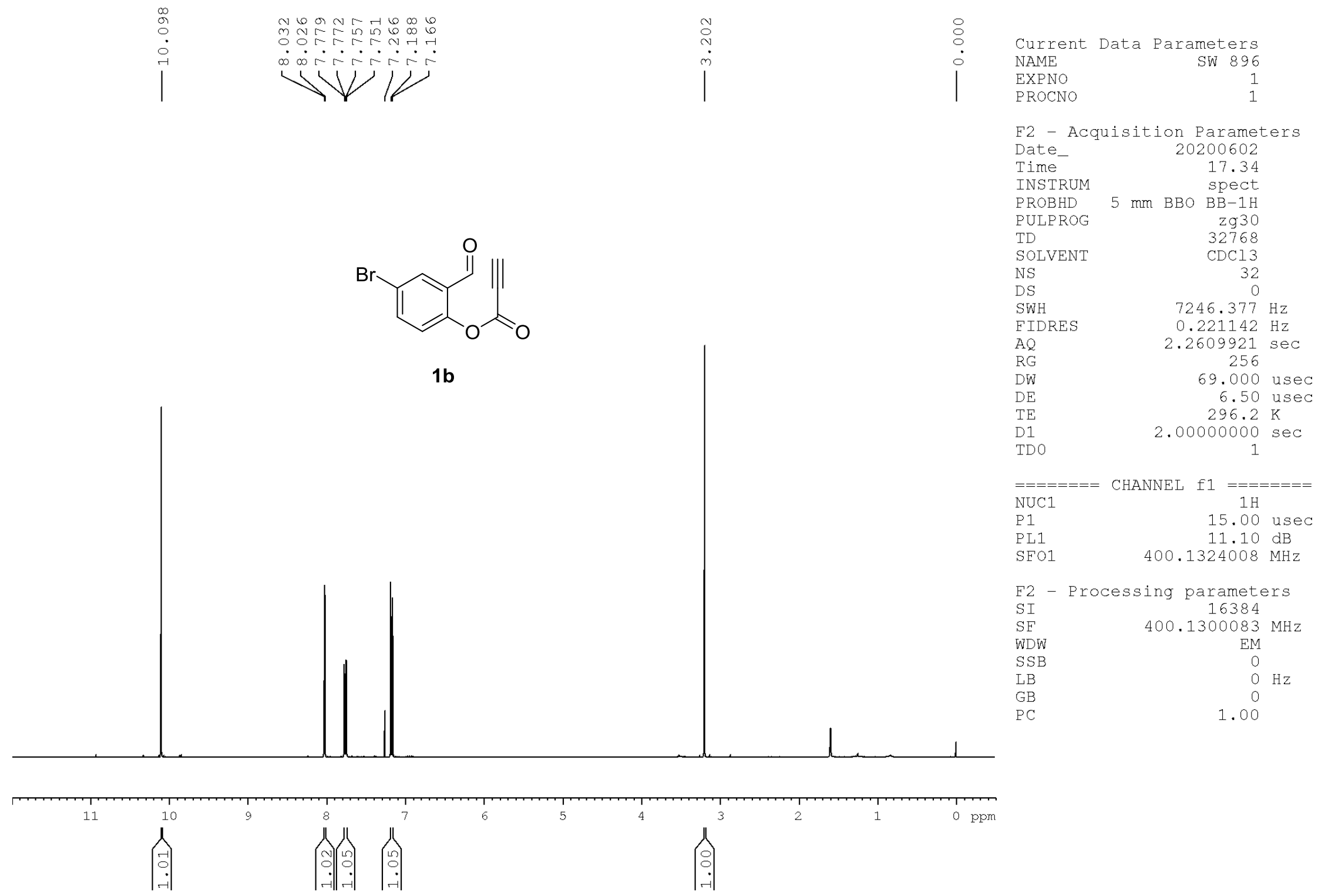




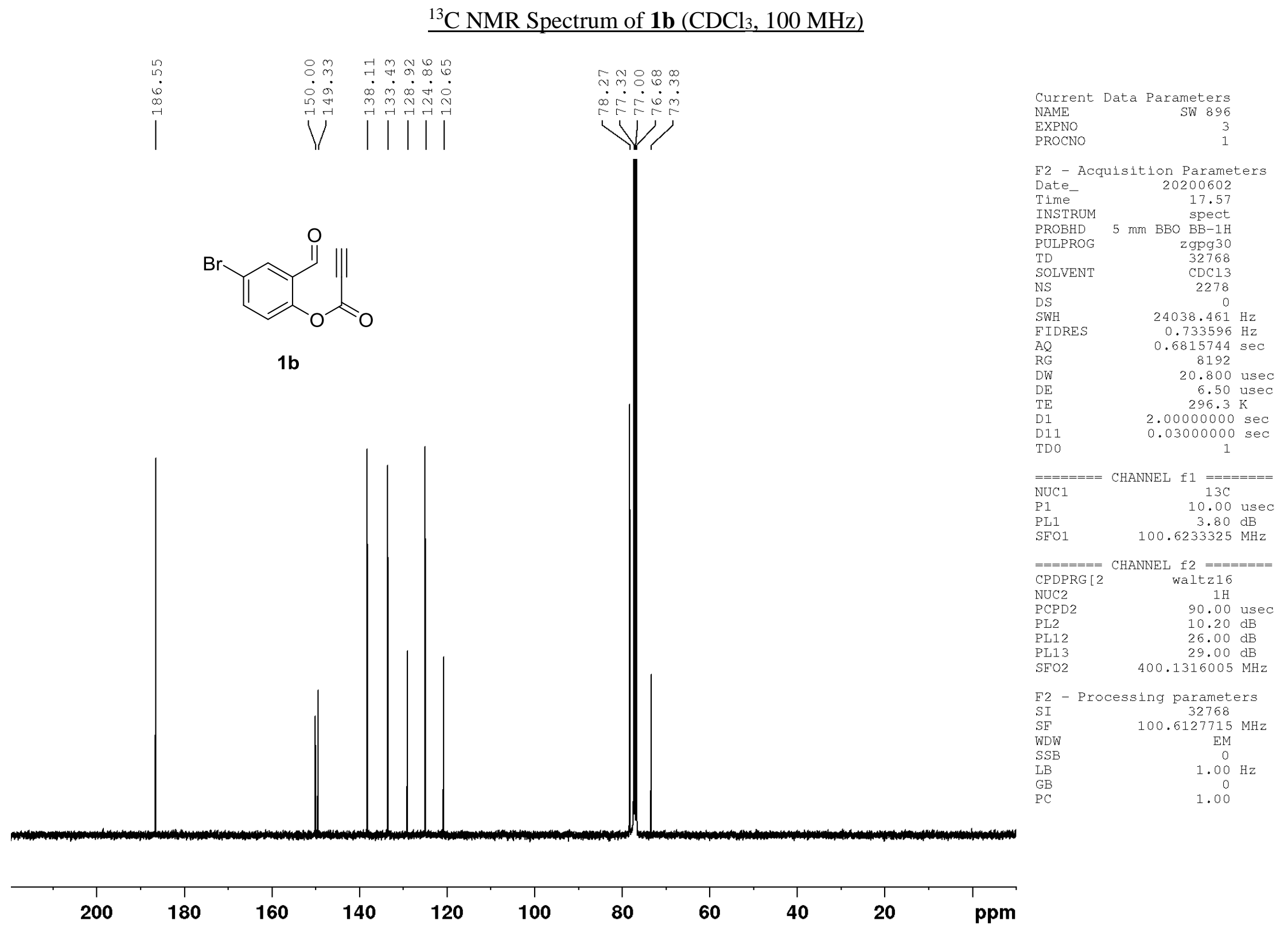


${ }^{1} \mathrm{H}$ NMR Spectrum of $\mathbf{1 c}\left(\mathrm{CDCl}_{3}, 400 \mathrm{MHz}\right)$

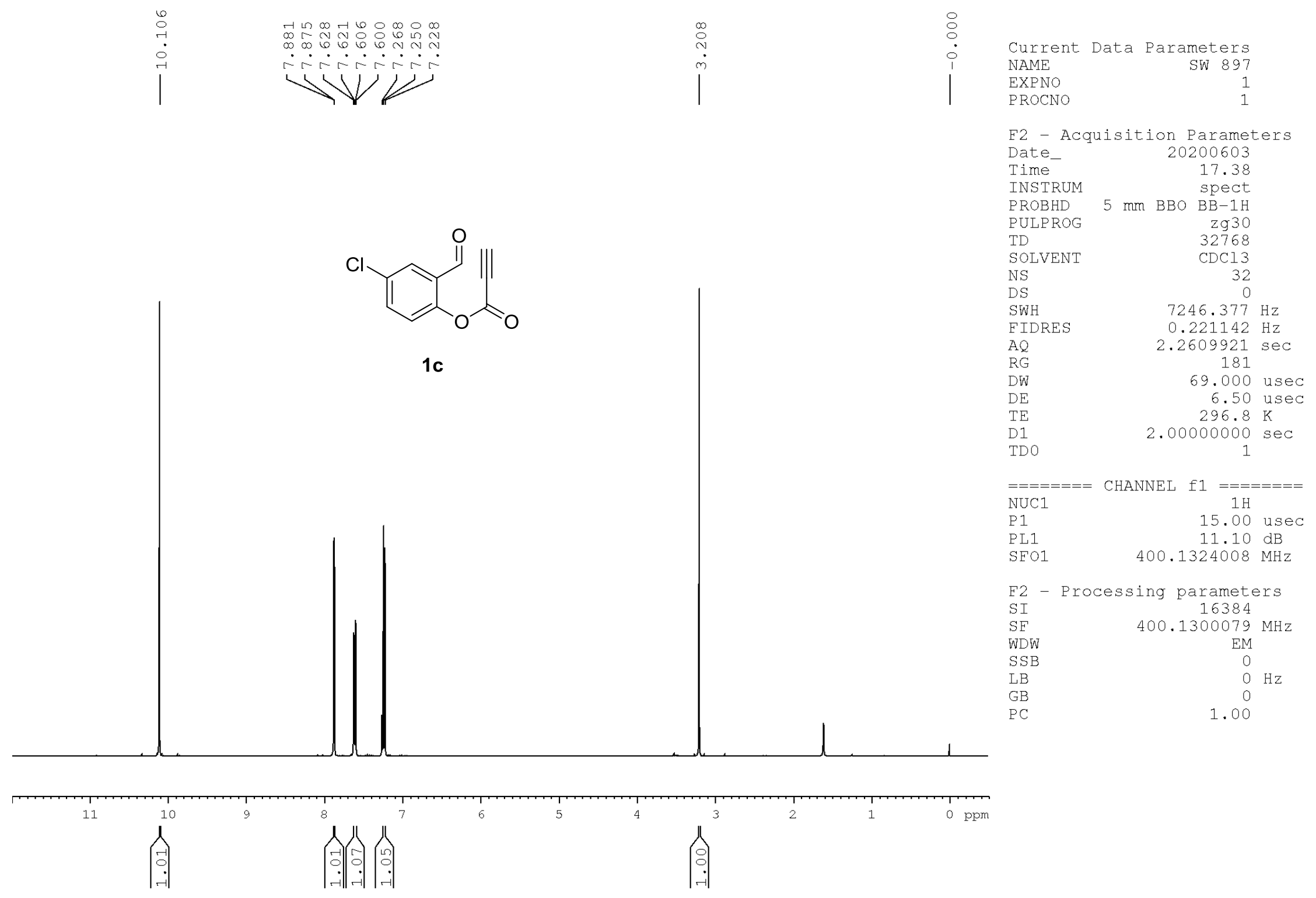




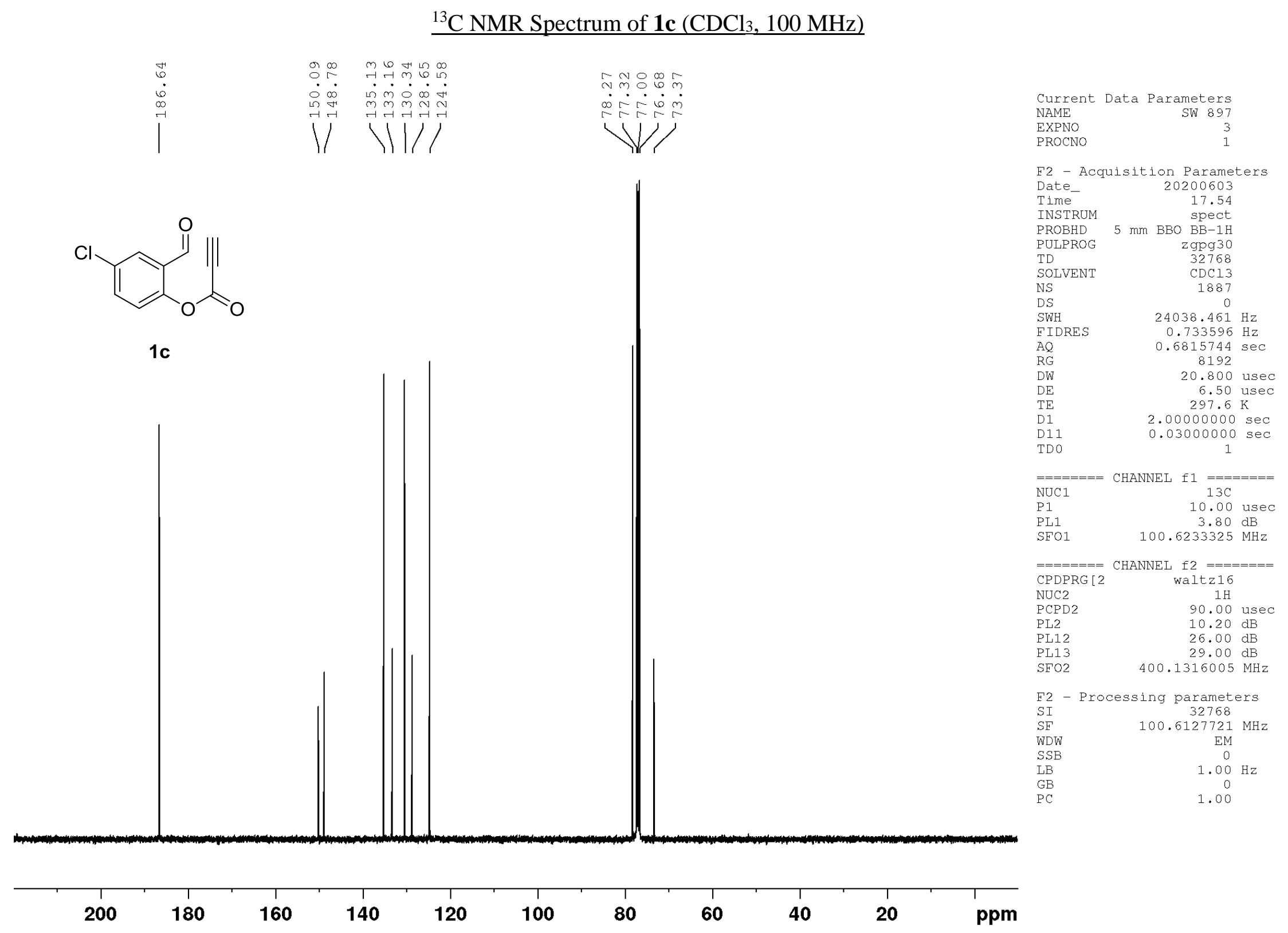


${ }^{1} \mathrm{H}$ NMR Spectrum of $\mathbf{1 d}\left(\mathrm{CDCl}_{3}, 400 \mathrm{MHz}\right)$

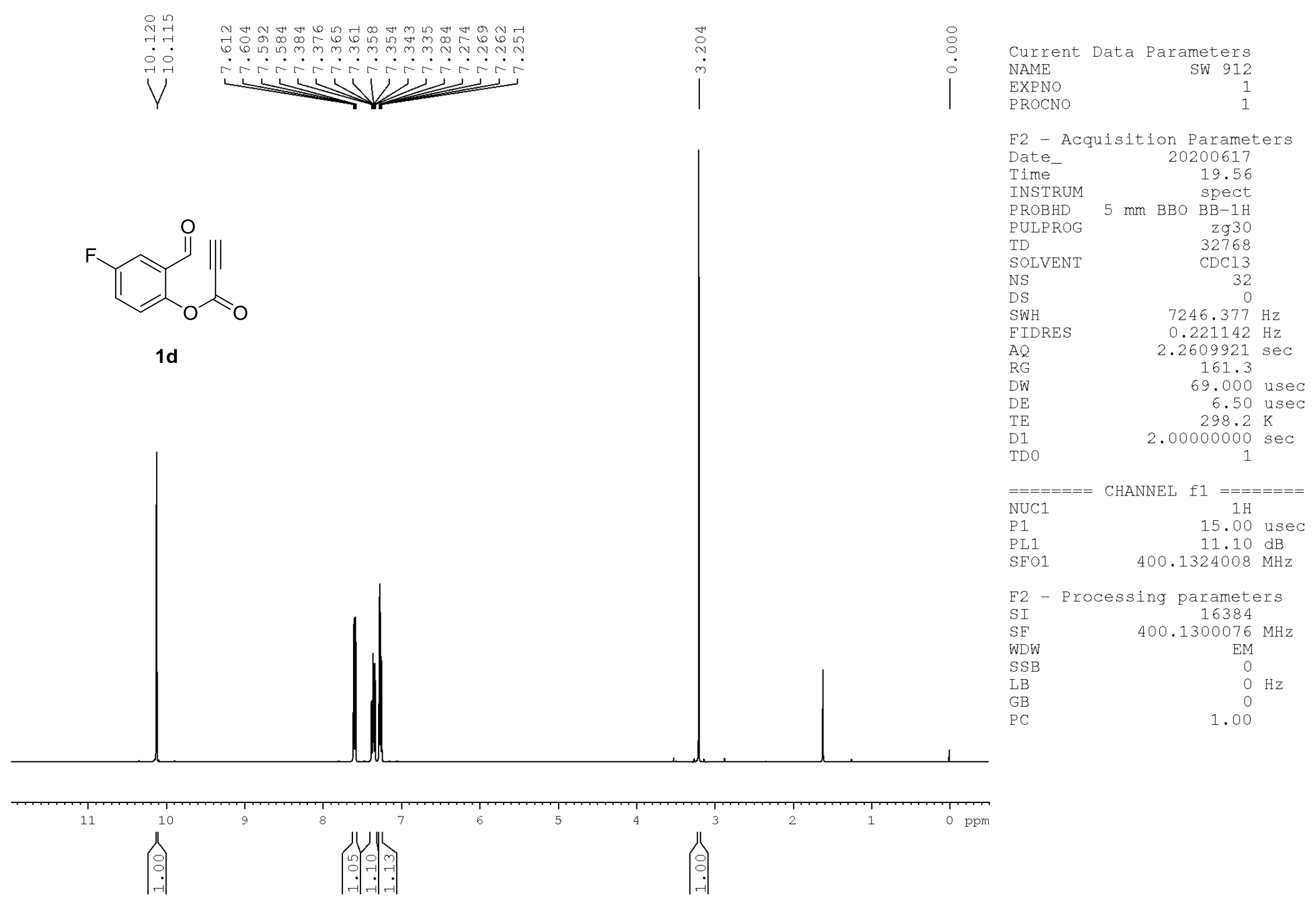




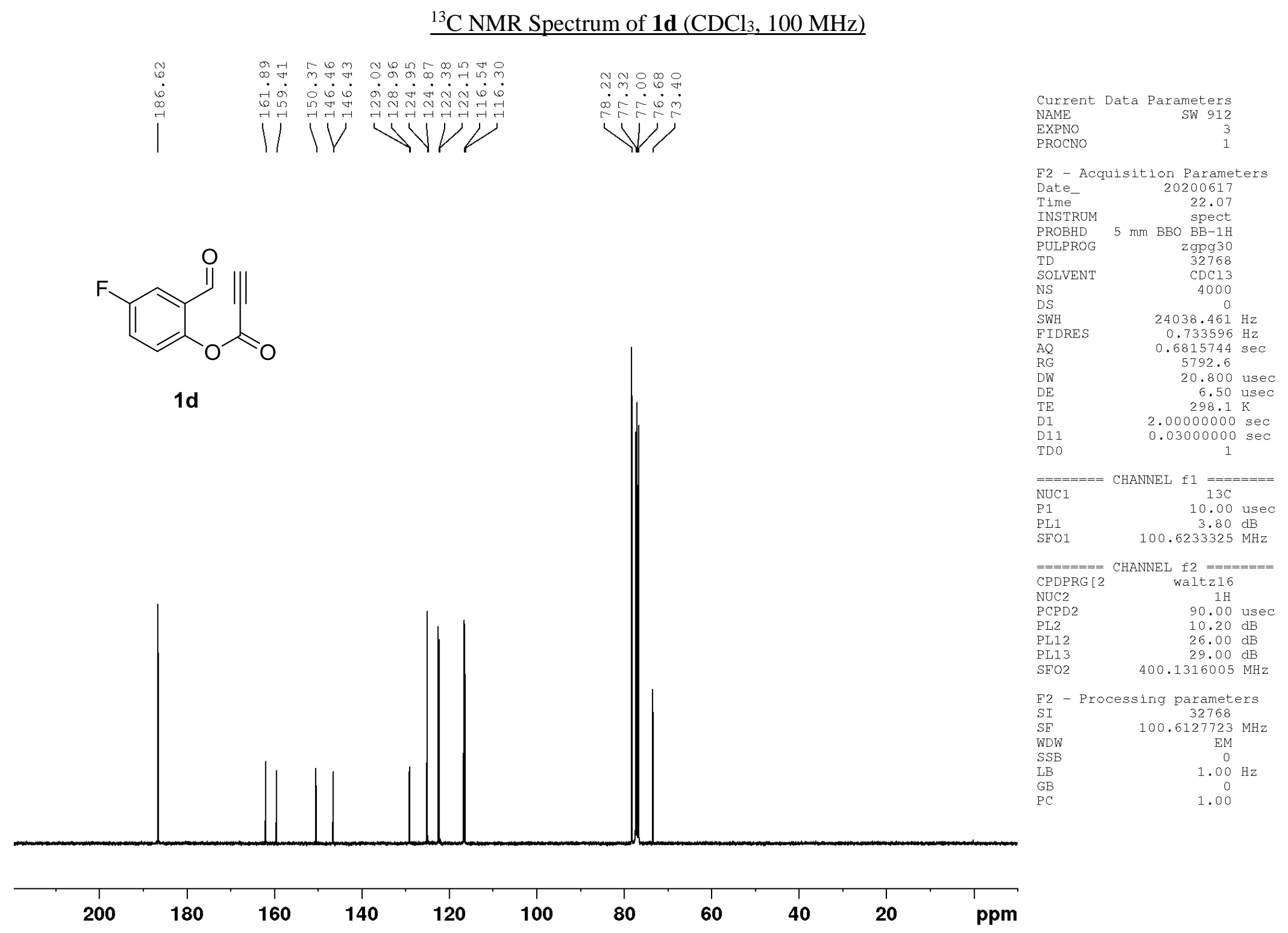




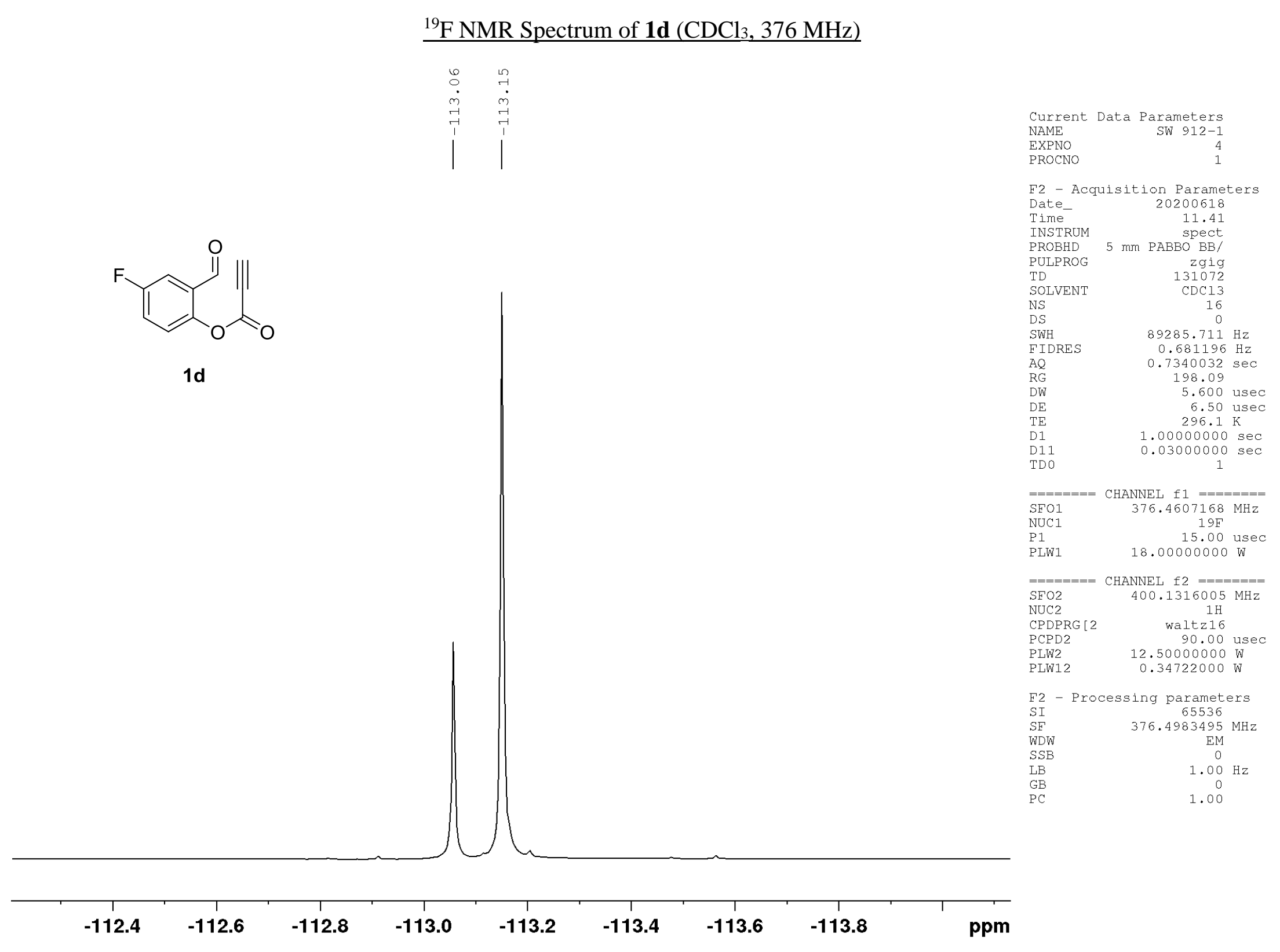


${ }^{1} \mathrm{H}$ NMR Spectrum of $1 \mathrm{e}\left(\mathrm{CDCl}_{3}, 400 \mathrm{MHz}\right)$

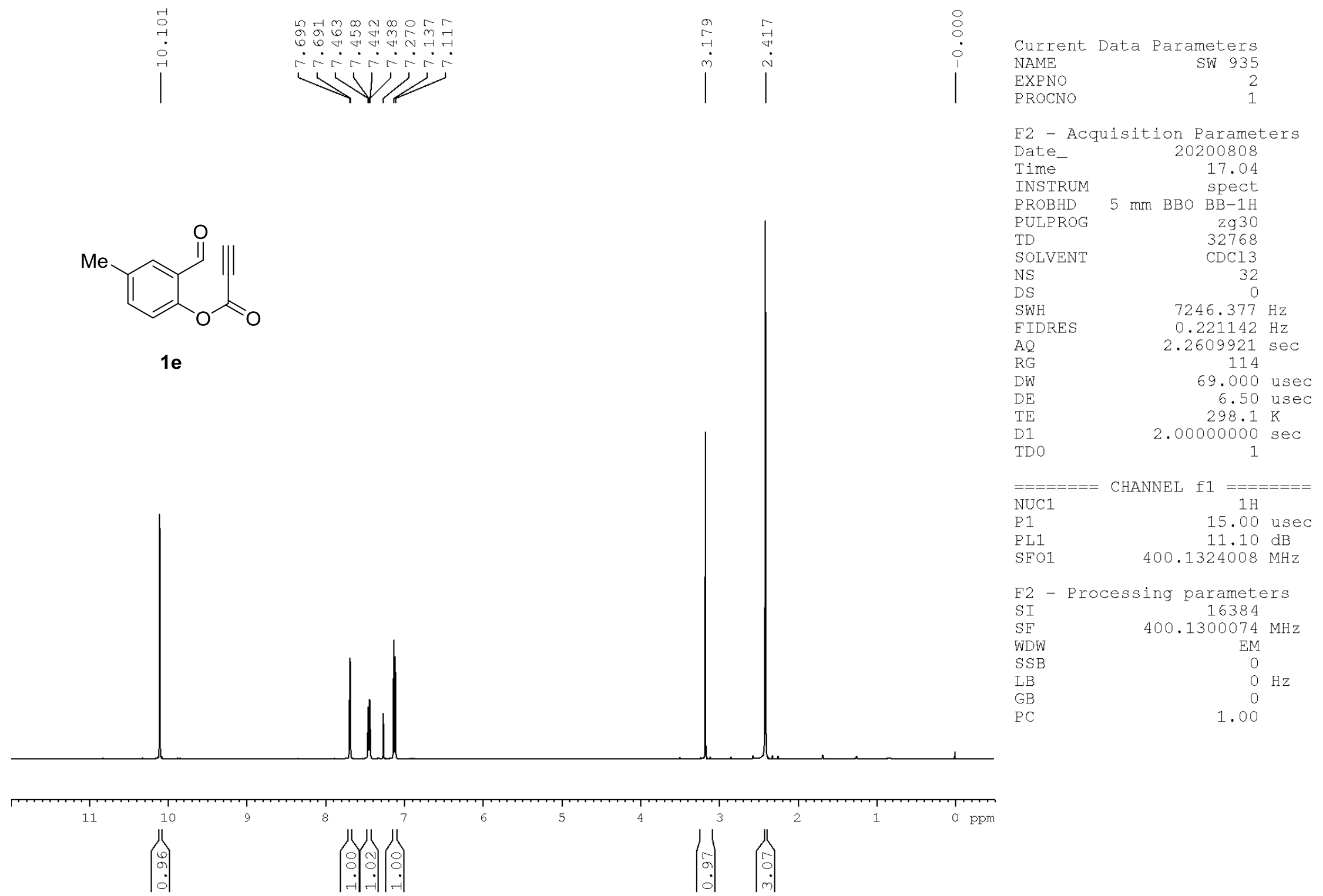


${ }^{13} \mathrm{C}$ NMR Spectrum of $1 \mathrm{e}\left(\mathrm{CDCl}_{3}, 100 \mathrm{MHz}\right)$
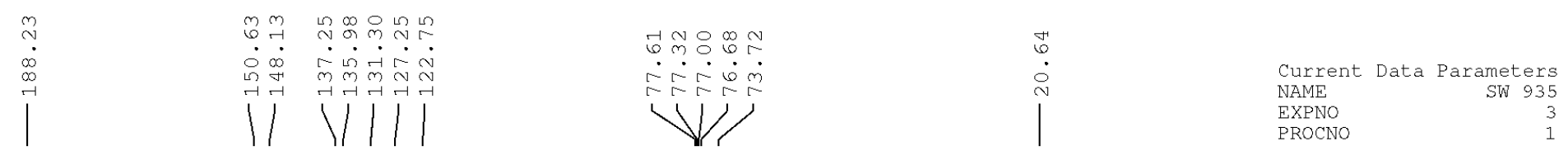

EXPNO
PROCNO

F2 - Acquisition Parameter

Date_ 20200808

Time

INSTRUM

PROBHD

17.07

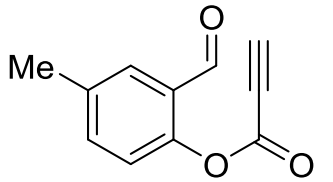

TD

SOLVEN

DS

SWH

$0.733596 \mathrm{~Hz}$
$0.6815744 \mathrm{sec}$

RG

DW

TE
D1
D11

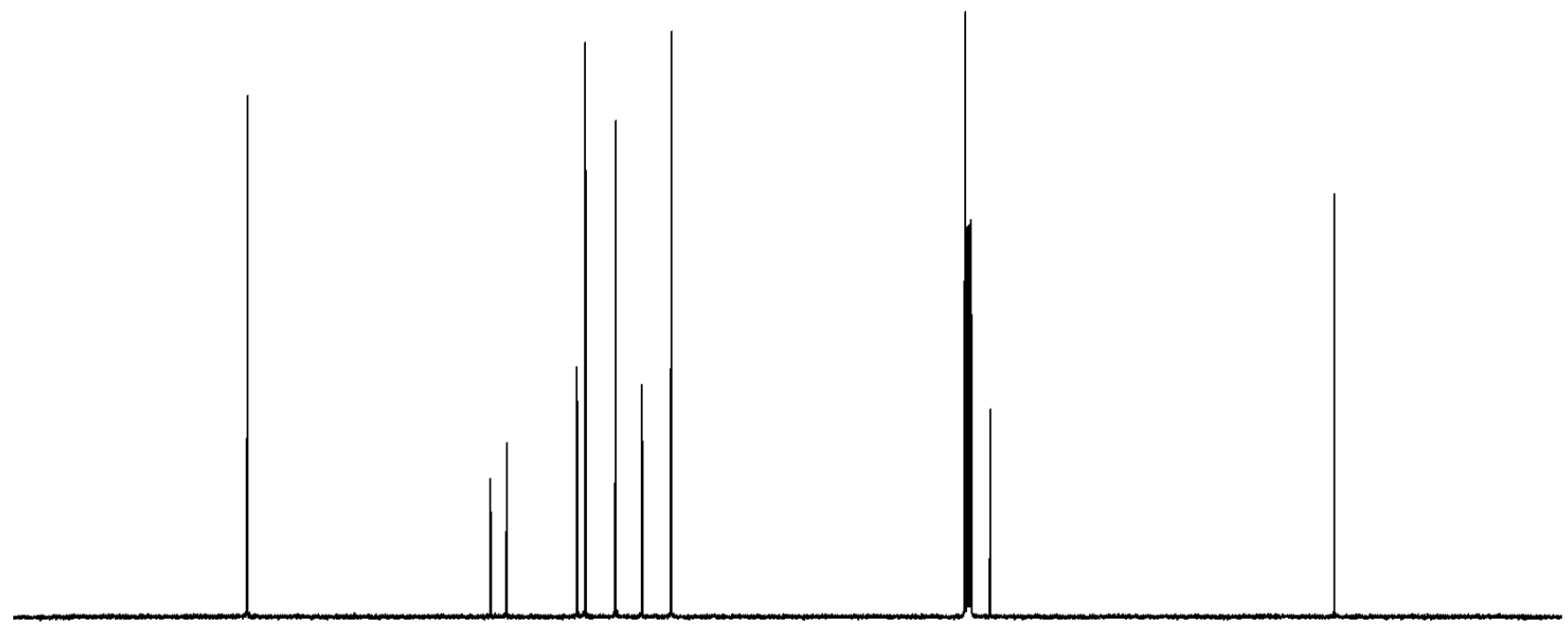

$====$
NUC1
P1

$13 \mathrm{C}$

$10.00 \mathrm{usec}$

PL1 $\quad 3.80 \mathrm{~dB}$

$========$ CHANNEL $\mathrm{f} 2 \quad========$

CPDPRG [2 waltz16

$\begin{array}{lr}\text { NCC2 } & 1 \mathrm{H} \\ \text { PCPD2 } & 90.00 \text { usec }\end{array}$

$10.20 \mathrm{~dB}$

$26.00 \mathrm{~dB}$
$29.00 \mathrm{~dB}$

PL13

$400.1316005 \mathrm{MHz}$

F2 - Processing parameters

$\begin{array}{lr}\text { SI } & 32768 \\ \text { SF } & 100.6127758 \mathrm{MHz}\end{array}$

WDW $\quad 100.6127758$

SSB

$\mathrm{GB}$

$\mathrm{EM}$
0
$1.00 \mathrm{~Hz}$

$1.00 \mathrm{H}$
0
1.00

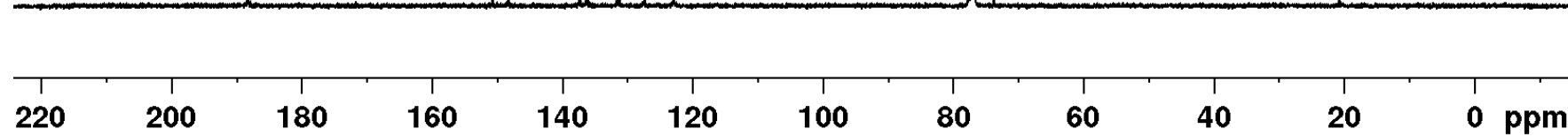


${ }^{1} \mathrm{H}$ NMR Spectrum of $\mathbf{1 f}\left(\mathrm{CDCl}_{3}, 400 \mathrm{MHz}\right)$

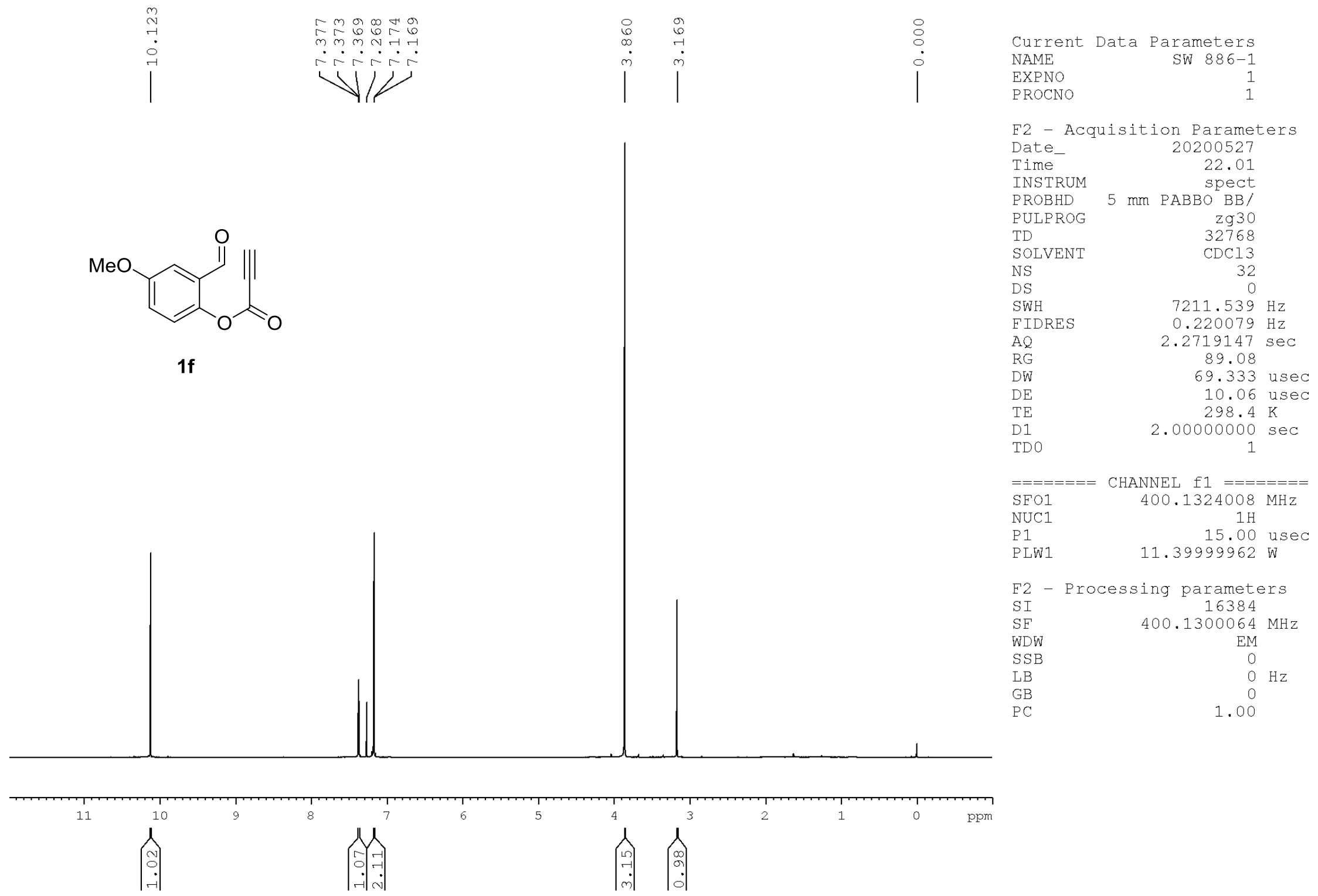




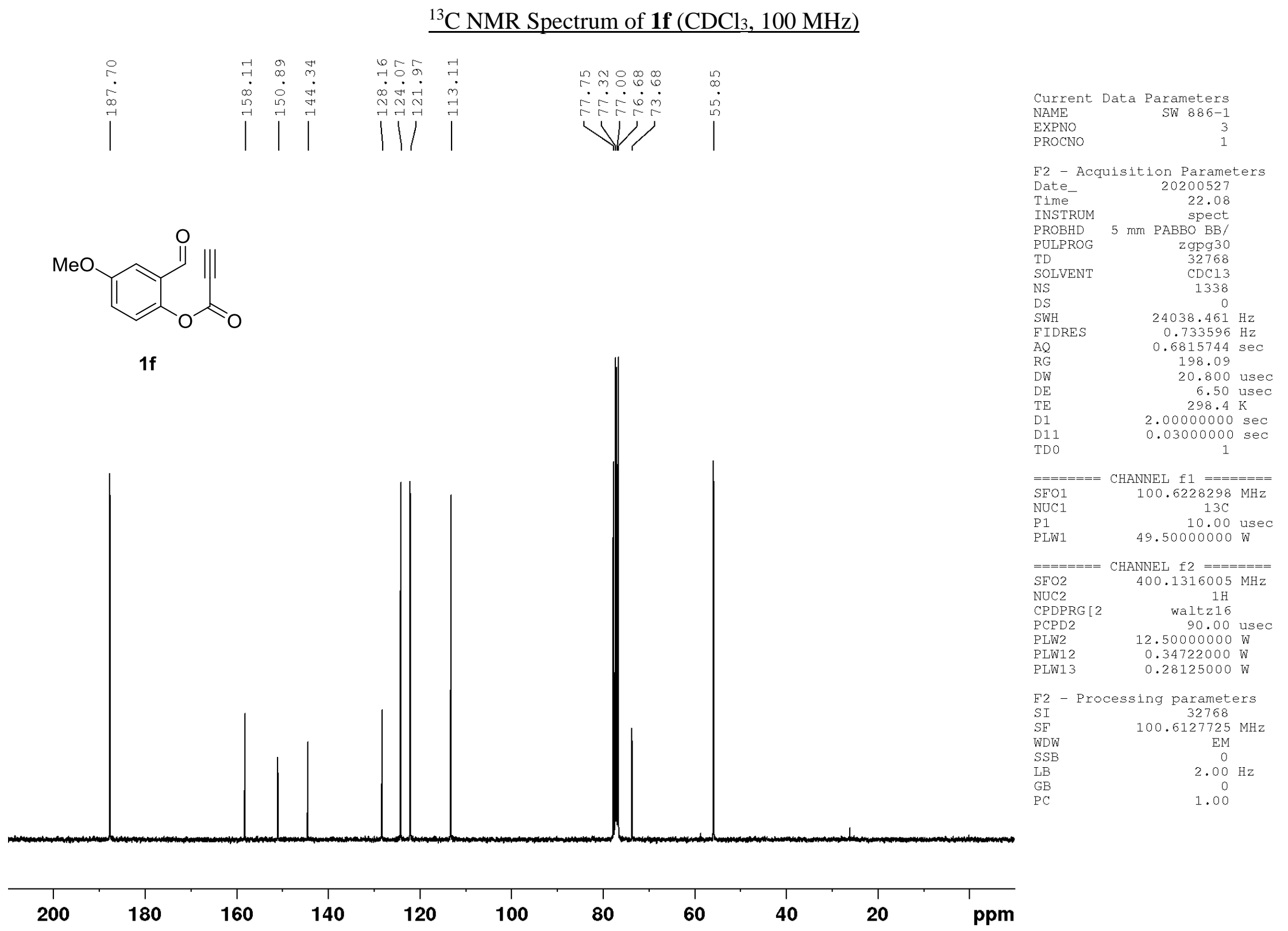


$\underline{{ }^{1} \mathrm{H} \text { NMR Spectrum of } 1 \mathrm{~g}\left(\mathrm{CDCl}_{3}, 400 \mathrm{MHz}\right)}$
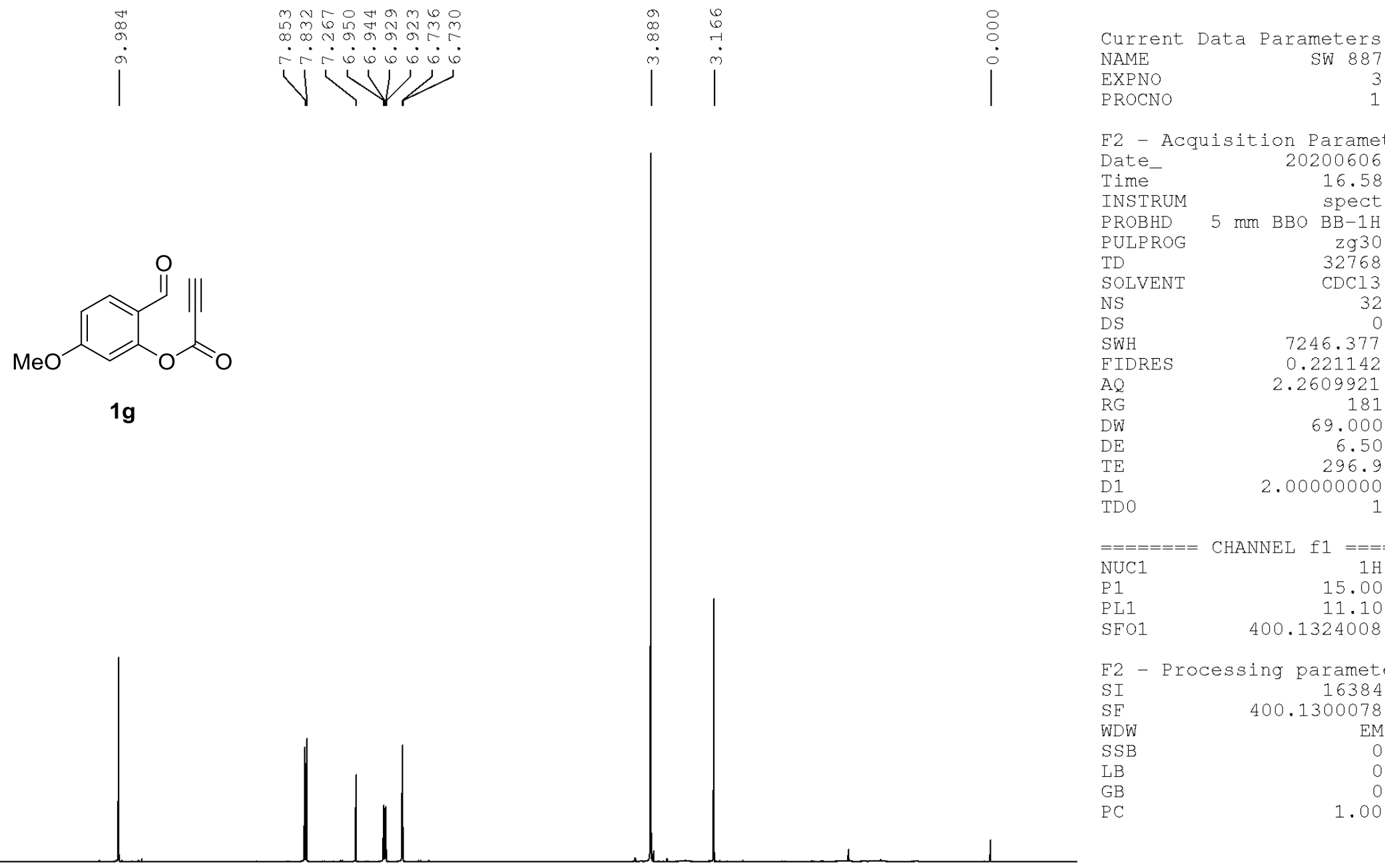

F2 - Acquisition Parameters

Date__ 20200606

16.58

INSTRUM

PROBHD

$5 \mathrm{~mm} \mathrm{BBO} \mathrm{BB}-1 \mathrm{H}$

PULPROG

$\mathrm{TD}$

SOLVENT

NS

DS

SWH

FIDRES

$\mathrm{AQ}$

$1 \mathrm{~g}$

DW

DE

E

TDO

$\mathrm{BB}-1 \mathrm{H}$

$\mathrm{zg} 30$
32768

$\mathrm{CDCl} 3$

$\mathrm{O}$
$7246.377 \mathrm{~Hz}$
$0.221142 \mathrm{~Hz}$

$0.221142 \mathrm{~Hz}$ 2.2609921 sec

69.000 usec

6.50 usec

$296.9 \mathrm{~K}$

$2.00000000 \mathrm{sec}$

$\begin{array}{lr}=======\text { CHANNEL } \mathrm{fl}======= \\ \text { NUC1 } & 1 \mathrm{H} \\ \text { P1 } & 15.00 \mathrm{usec} \\ \text { PL1 } & 11.10 \mathrm{~dB} \\ \text { SE01 } & 1324008 \mathrm{mHz}\end{array}$

400.1324008 dB

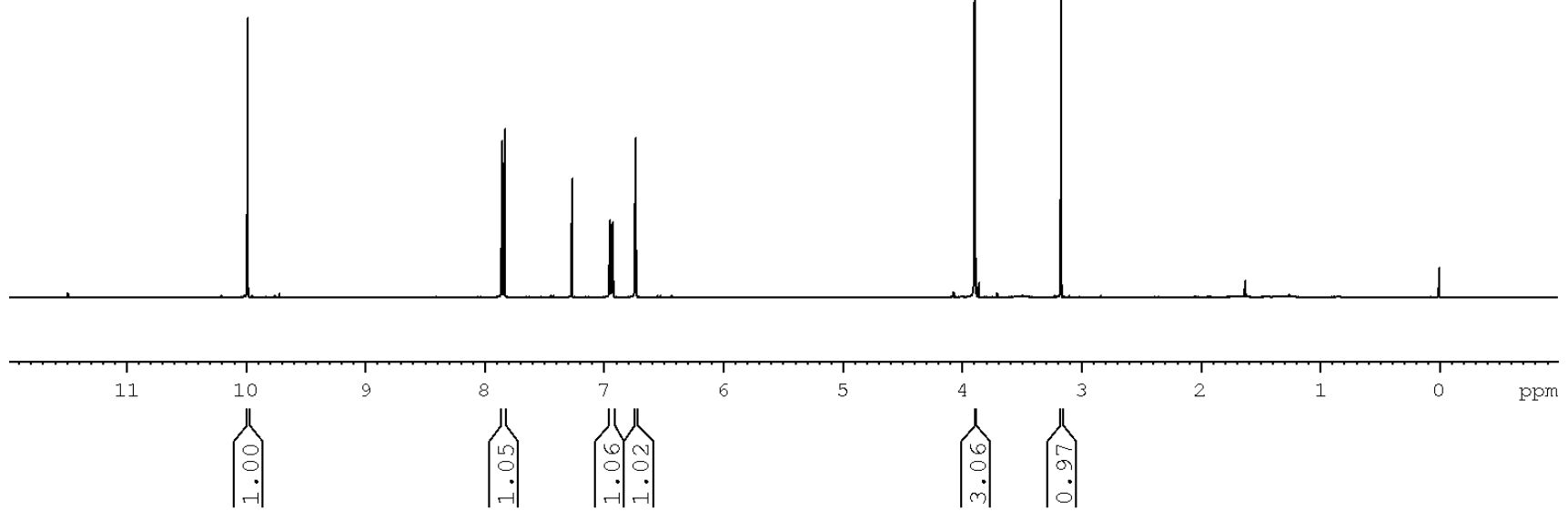

F2 - Processing parameters

16384

SF $\quad 400.1300078 \mathrm{MHz}$

WDW

SSB
LB

LB

$\mathrm{PC}$ EM 
${ }^{13} \mathrm{C}$ NMR Spectrum of $1 \mathrm{~g}\left(\mathrm{CDCl}_{3}, 100 \mathrm{MHz}\right)$

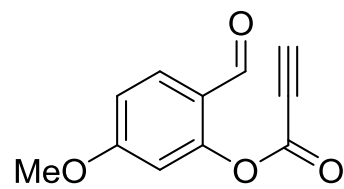

$1 \mathrm{~g}$

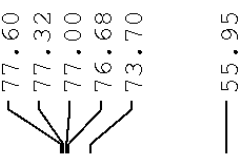

Current Data Parameters

NAME

PROCNO

SW 887

F2 - Acquisition Parameters

Date__ 20200606

Time

INSTRUM

PROBHD

17.00

BB $-1 \mathrm{H}$

SOLVE

NS

SWH

FIDRE

RG

DW

TE 1

D 11

$===$

NUTC

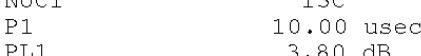

PL1 $\quad 3.80 \mathrm{~dB}$

$=======$ CHANNTL

CPDPRG [2 waltz16

NUC2 $21 \mathrm{H}$ usec

PL12 $26.00 \mathrm{~dB}$

$29.00 \mathrm{~dB}$

SFO2 $400.1316005 \mathrm{MHz}$

F2 - Processing parameters

$\begin{array}{lr}\text { SI } & 32768 \\ \text { SF } & 100.6127723 \mathrm{MH}\end{array}$

SF
WDW
SSB

SSB
LB

$\mathrm{LB}$
$\mathrm{PC}$

$\mathrm{EM}$
0

$1.00 \mathrm{~Hz}$

1.00

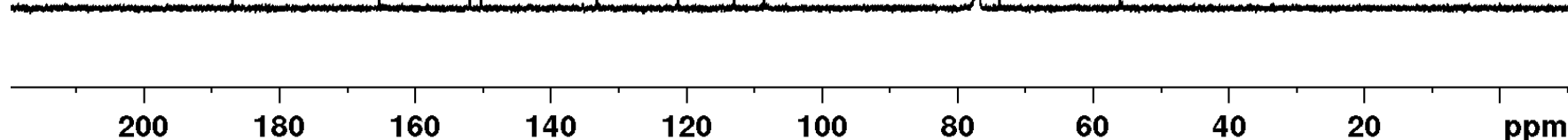


$\underline{{ }^{1} \mathrm{H} \text { NMR Spectrum of } \mathbf{1 h}\left(\mathrm{CDCl}_{3}, 400 \mathrm{MHz}\right)}$

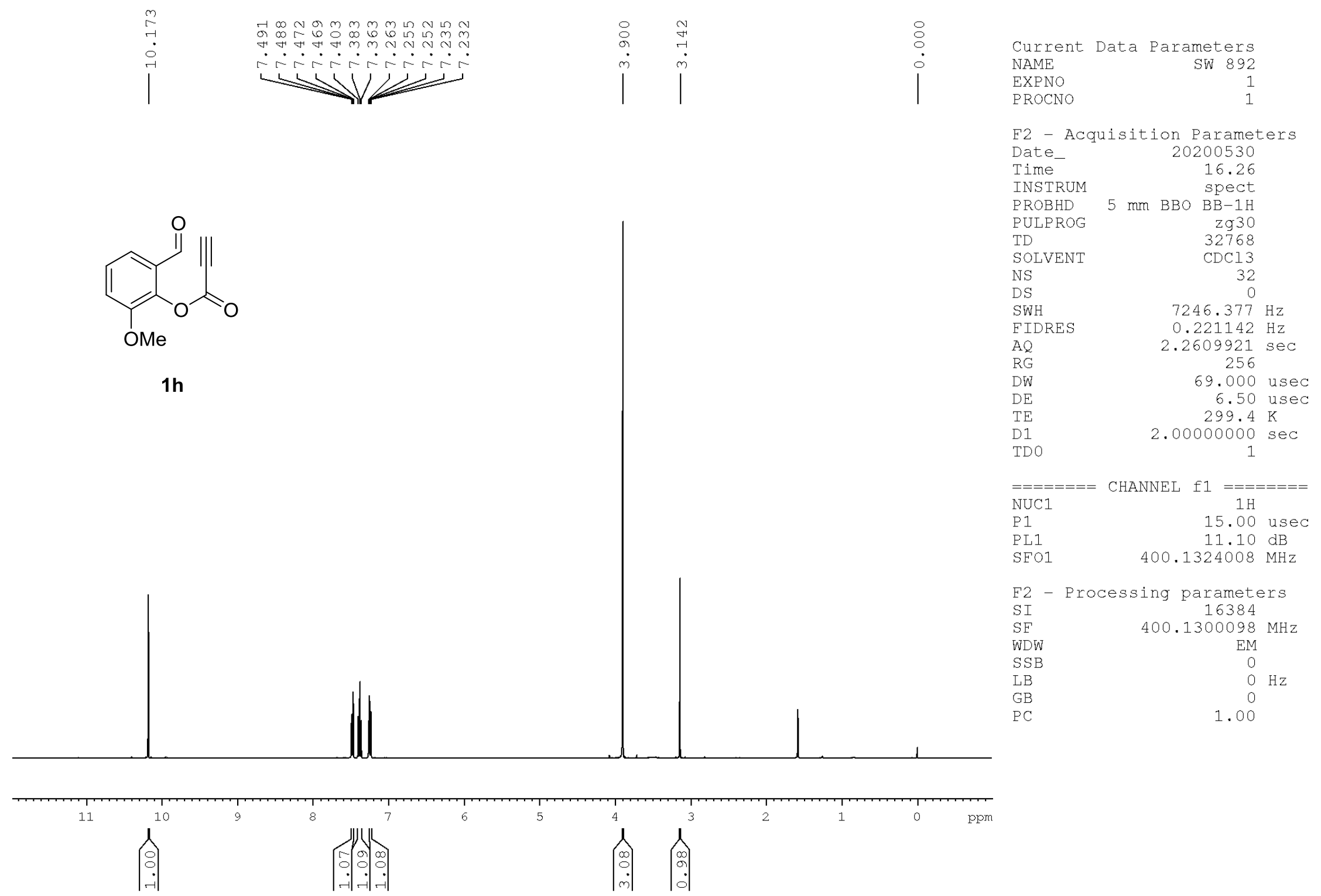


${ }^{13} \mathrm{C}$ NMR Spectrum of $\mathbf{1 h}\left(\mathrm{CDCl}_{3}, 100 \mathrm{MHz}\right)$
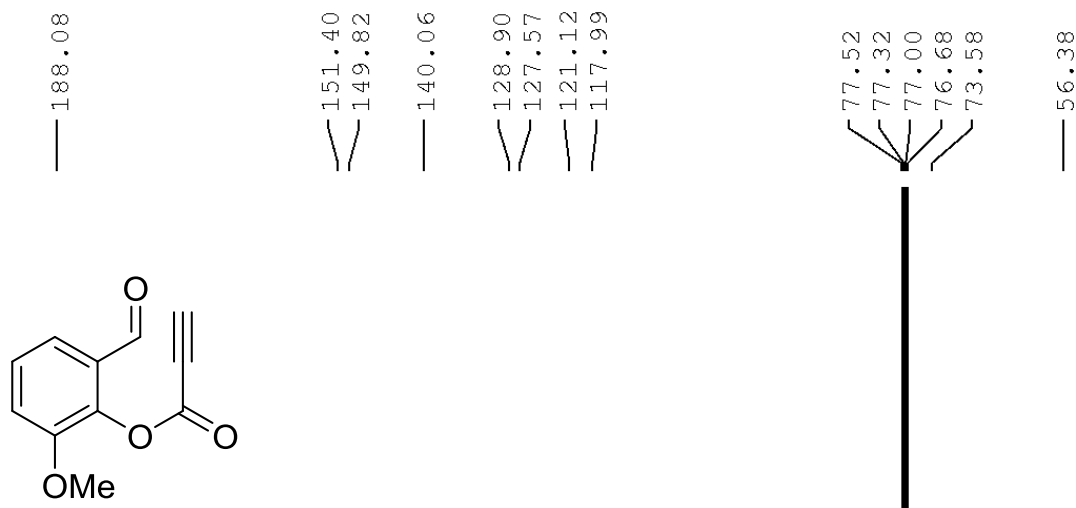

Current Data Parameters

NAME

EROCNO

SW 892-1

F2 - Acquisition Parameters

Date__ 20200530

INSTRUM spect

PROBHD $5 \mathrm{~mm}$ PABBO BB/

PULPROG $\quad$ zgpg30

SOLVE
NS

DS

$24038.461 \mathrm{~Hz}$

FIDRES $\quad 0.733596 \mathrm{~Hz}$

AQ $\quad 0.6815744 \mathrm{sec}$

RG

$1 \mathrm{~h}$

198.09
20.800 usec

20.800 usec
6.50 usec

$\begin{array}{lr}\mathrm{DE} & 297.0 \\ \mathrm{TE} & \end{array}$

D1 $\quad 2.00000000 \mathrm{sec}$

TDO

$=======$ CHANNEL $\mathrm{f} 1 \quad========$
SFO1 $\quad 100.6228298 \mathrm{MHz}$

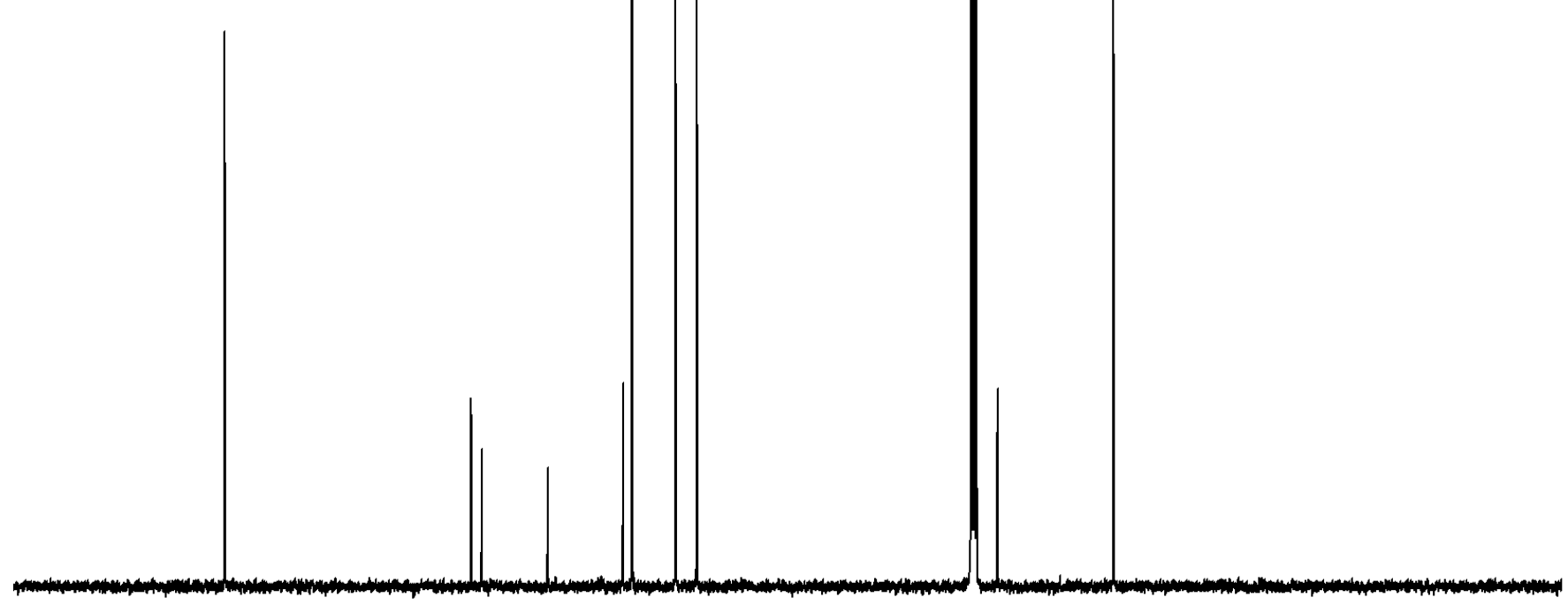

PLW 1

10.00 use

SFO2 $==$ CHANNEL $f 2$ $=======$

$\operatorname{sic} 2$

CPDPRG [

PCPD2

PLW12

PLW13 waltz16

90.00 use

$12.50000000 \mathrm{~W}$

$0.34722000 \mathrm{~W}$

2 - Processing paraneters

SI $\quad 32768$

WDW

$\begin{array}{lc}\mathrm{SSB} & 0 \\ \mathrm{LB} & 2.00 \mathrm{~Hz}\end{array}$

PB

0
1.00 
$\underline{{ }^{1} \mathrm{H} N M R}$ Spectrum of $1 \mathbf{i}\left(\mathrm{CDCl}_{3}, 400 \mathrm{MHz}\right)$

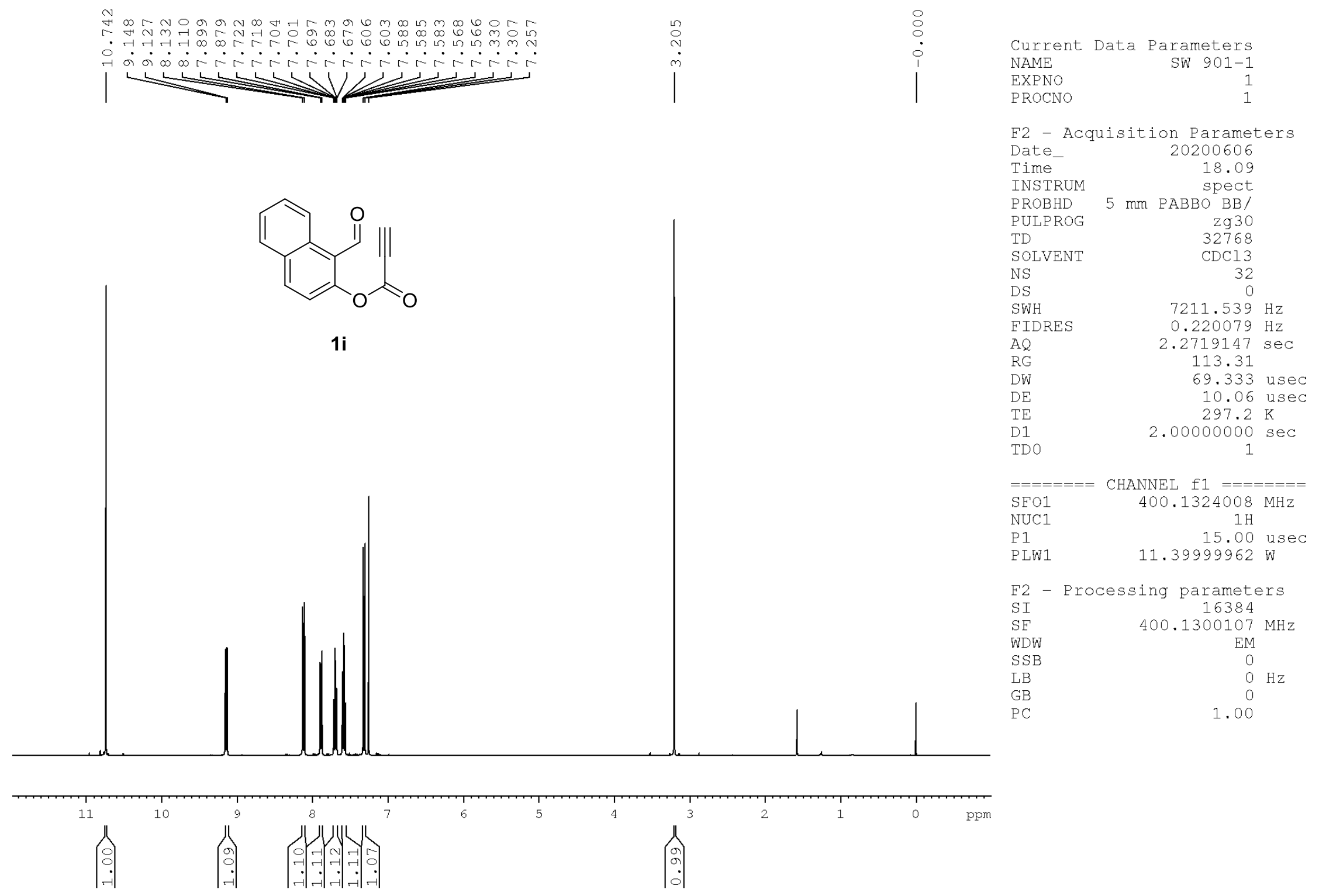


${ }^{13} \mathrm{C}$ NMR Spectrum of $\mathbf{1 i}\left(\mathrm{CDCl}_{3}, 100 \mathrm{MHz}\right)$
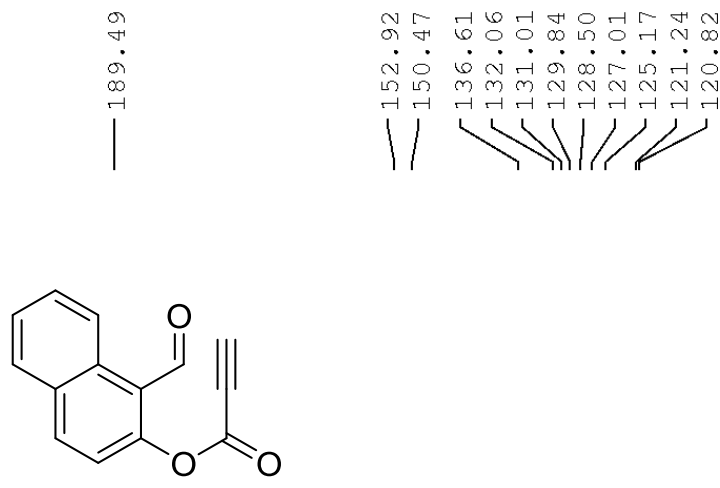

$1 i$
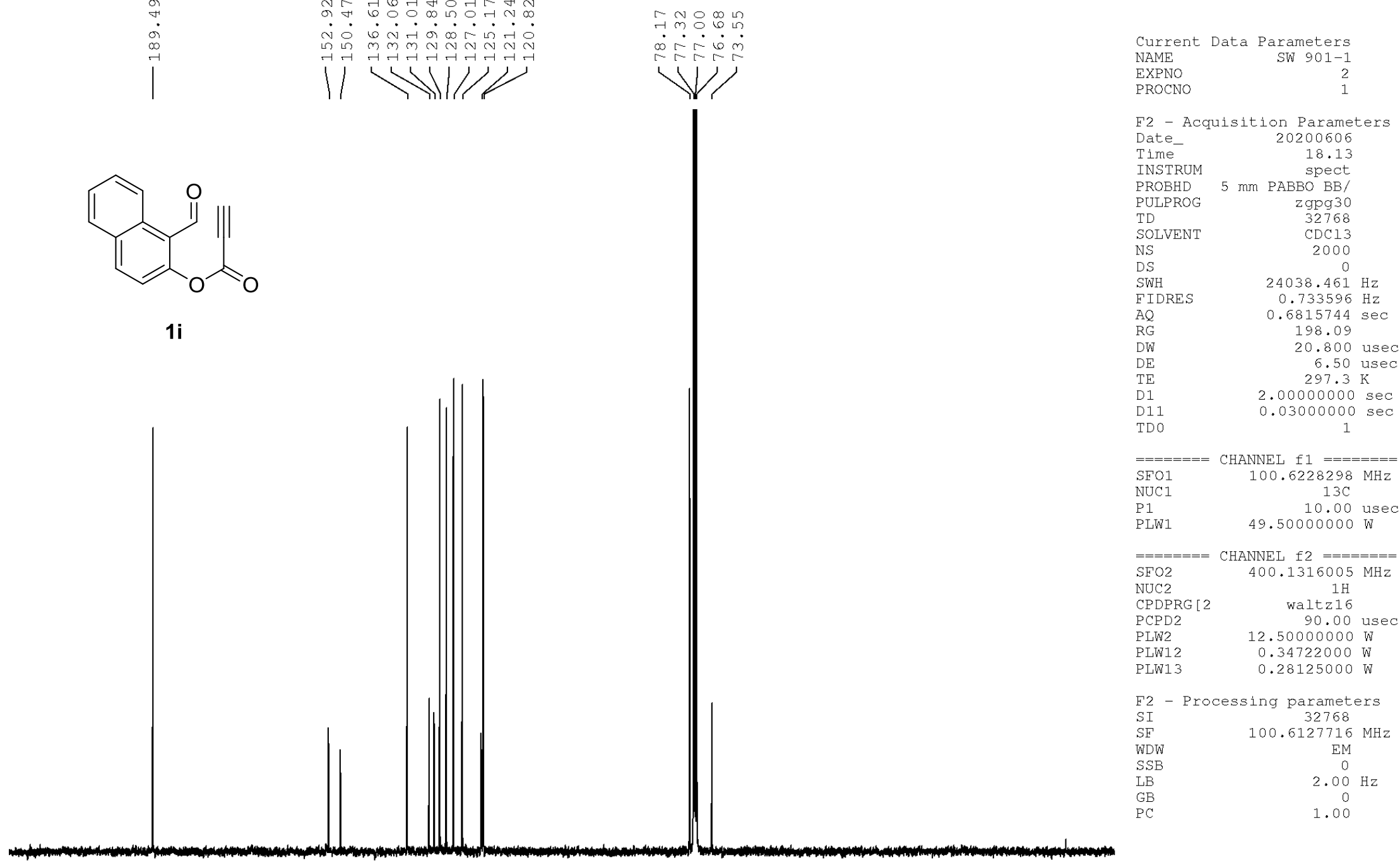
$\underline{{ }^{1} \mathrm{H} \text { NMR Spectrum of } \mathbf{9 a}\left(\mathrm{CDCl}_{3}, 400 \mathrm{MHz}\right)}$

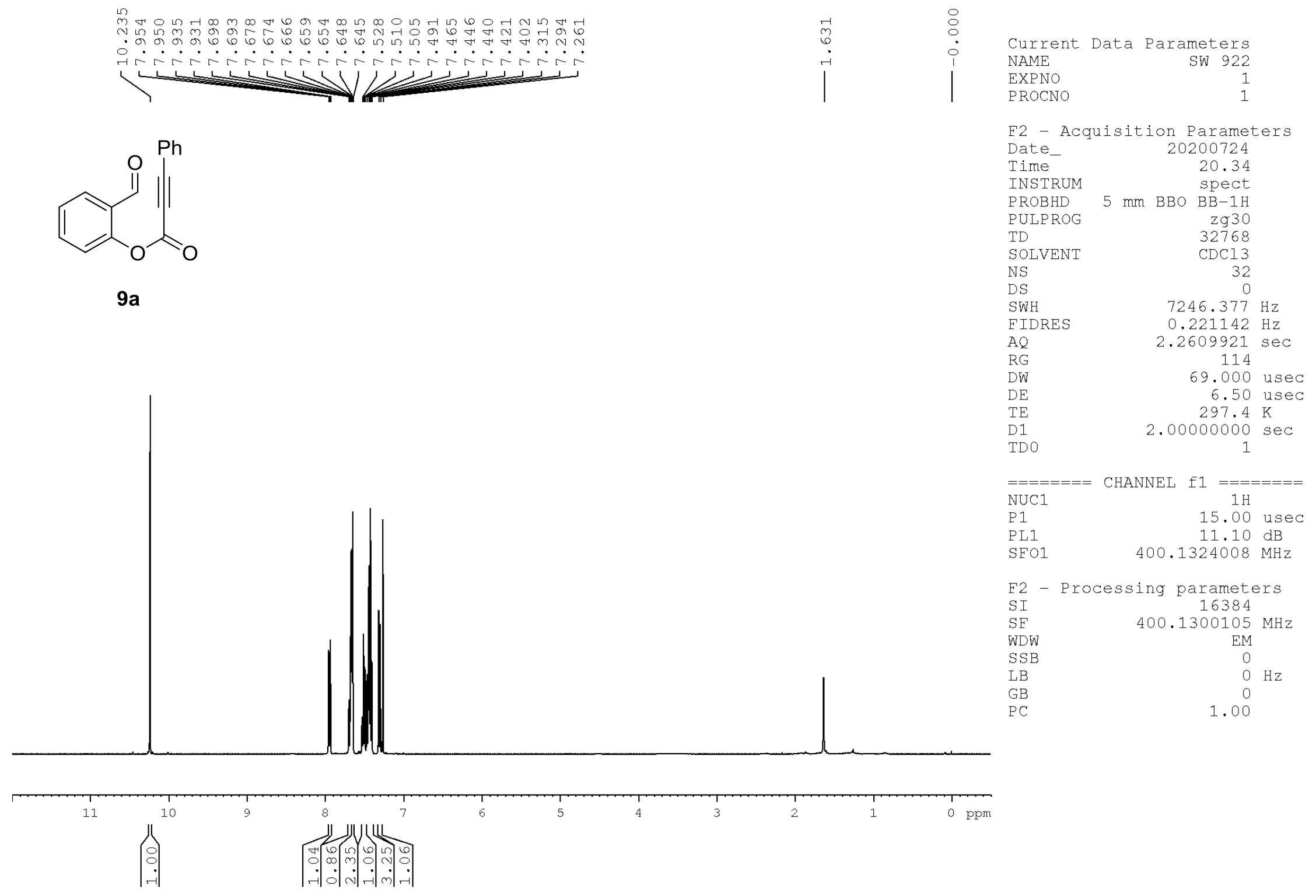


${ }^{13} \mathrm{C}$ NMR Spectrum of $9 \mathbf{a}\left(\mathrm{CDCl}_{3}, 100 \mathrm{MHz}\right)$

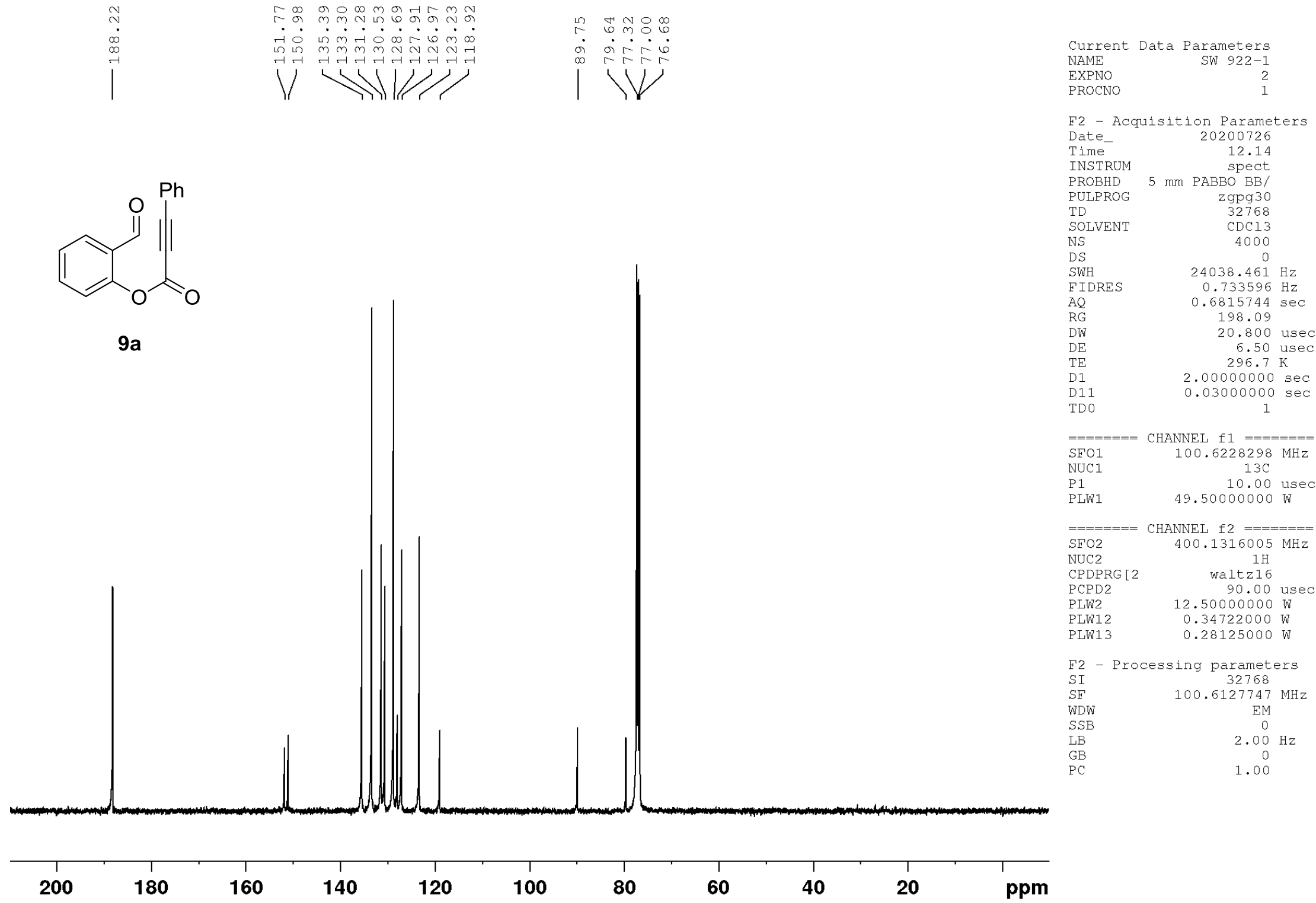




\section{$\underline{{ }^{1} \mathrm{H} \text { NMR Spectrum of } 9 \mathrm{~b}\left(\mathrm{CDCl}_{3}, 400 \mathrm{MHz}\right)}$}
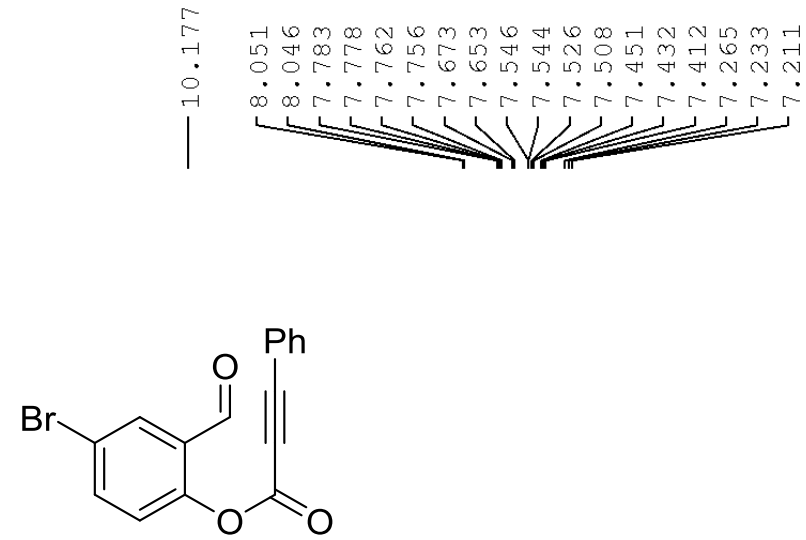

$9 b$

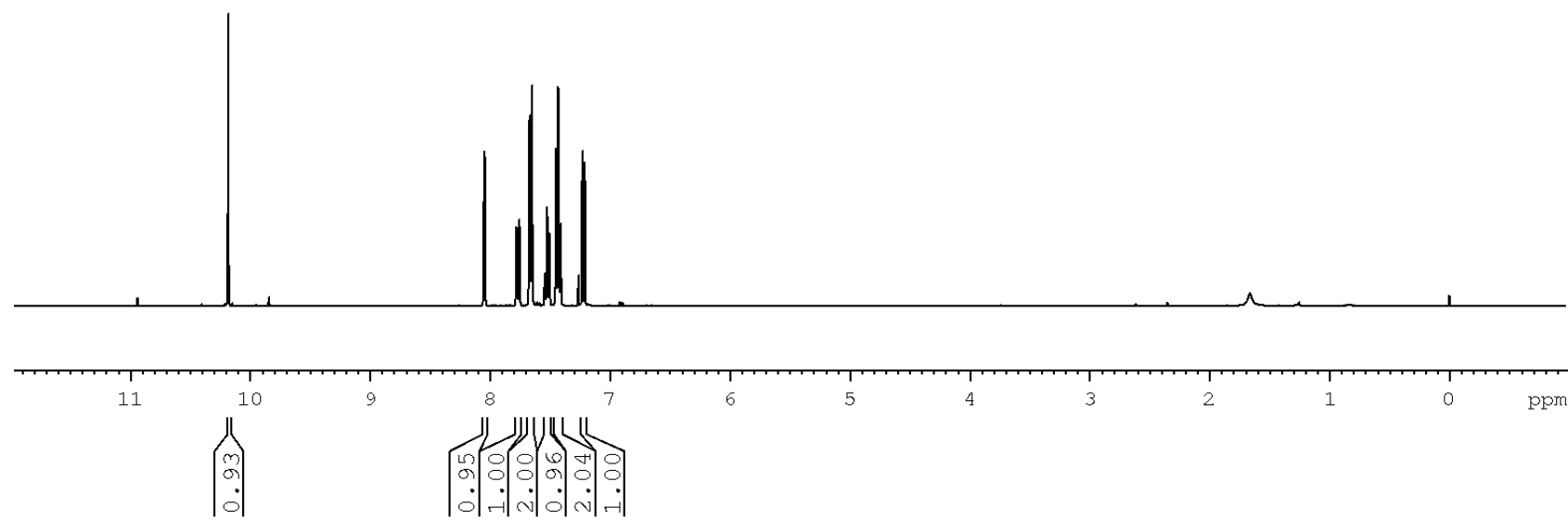

$\begin{array}{lr}\text { Current Data } & \text { Parameters } \\ \text { NAME } & \text { SW } \\ \text { EXPNO } & 1 \\ \text { PROCNO } & 1\end{array}$

F2 - Acquisition Parameter

Date_ 20200811

Time $\quad 16.56$

INSTRUM spect

PROBHD $5 \mathrm{~mm}$ PABBO BB/

PULPROG $\quad$ zg30

TD 32768

SOLVENT CDCl3

NS

DS

SWH $7211.539 \mathrm{~Hz}$

FIDRES $\quad 0.220079 \mathrm{~Hz}$

AO $\quad 2.2719147 \mathrm{~Hz}$

$\begin{array}{ll}\text { RG } & 89.08\end{array}$

DW 69.333 usec

DE $\quad 10.06$ usec

TE $292.3 \mathrm{~K}$

D1 $\quad 2.00000000 \mathrm{sec}$

$===$

CHANNEL f1 $=======$

$\mathrm{SFO1}$

$400.1324008 \mathrm{MHz}$ $1 \mathrm{H}$

PLW1

115.00 usec

2 - Processing parameters

SI 16384

SF $\quad 400.1300076 \mathrm{MHz}$

WDW

$S S B$
$L B$

GB

$\mathrm{PC}$ 
${ }^{13} \mathrm{C}$ NMR Spectrum of $9 \mathbf{b}\left(\mathrm{CDCl}_{3}, 100 \mathrm{MHz}\right)$
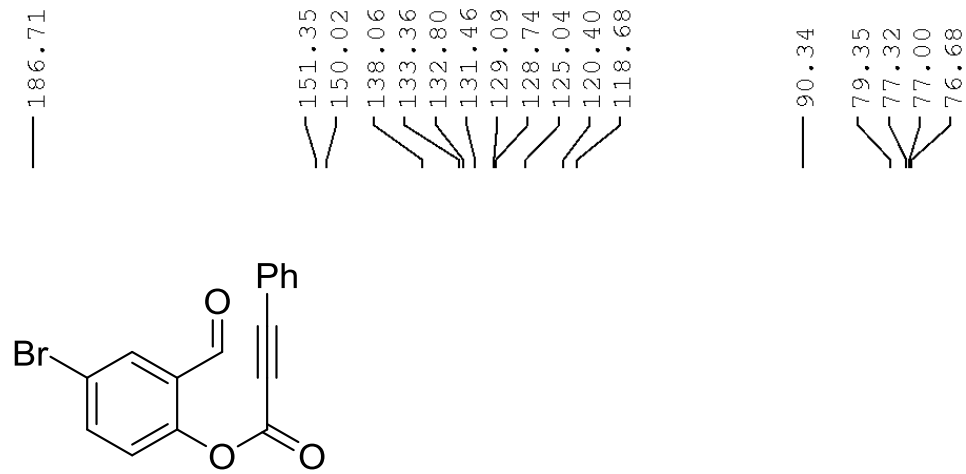

$9 b$
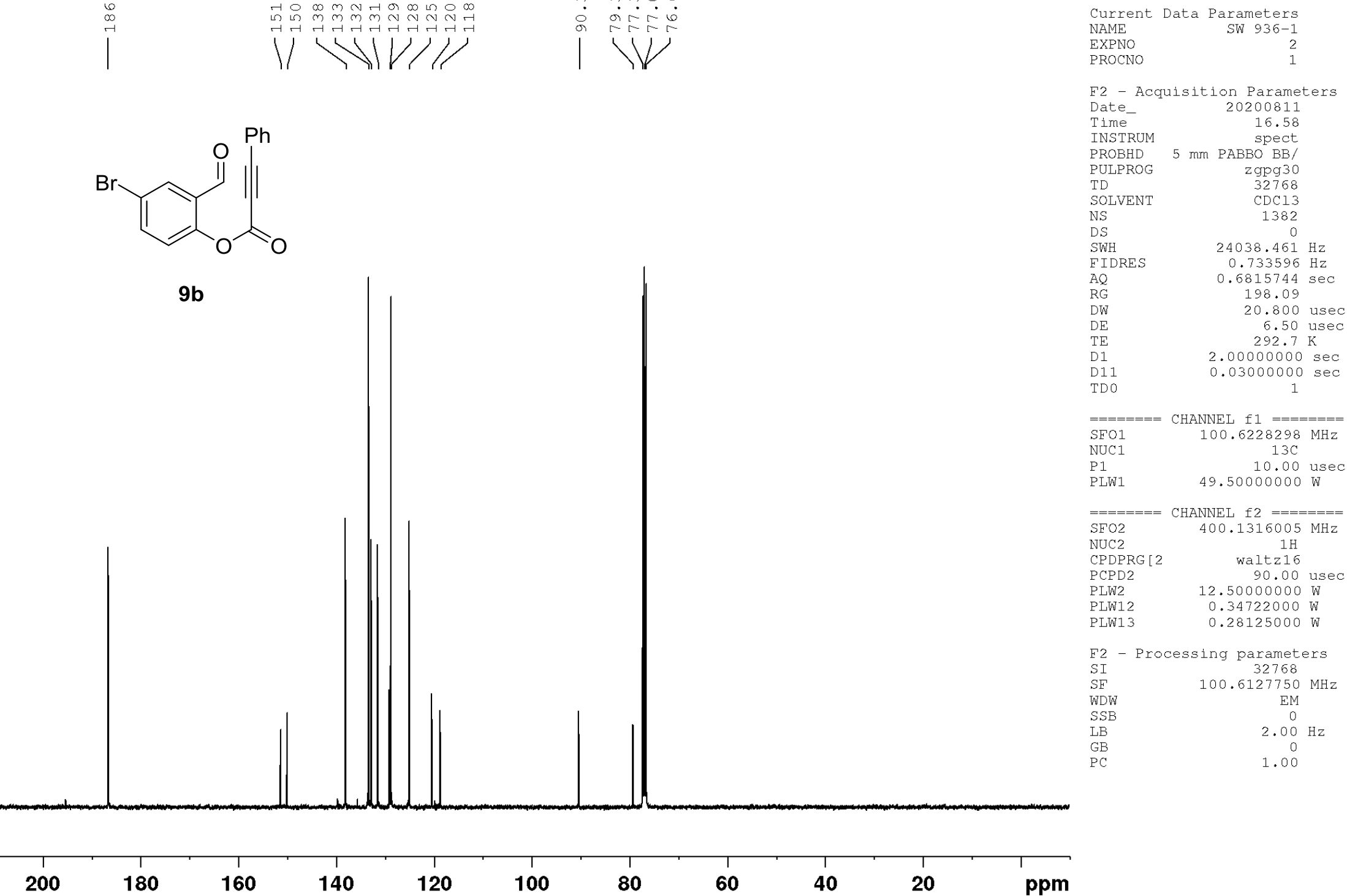
${ }^{1} \mathrm{H}$ NMR Spectrum of $9 \mathrm{c}\left(\mathrm{CDCl}_{3}, 400 \mathrm{MHz}\right)$
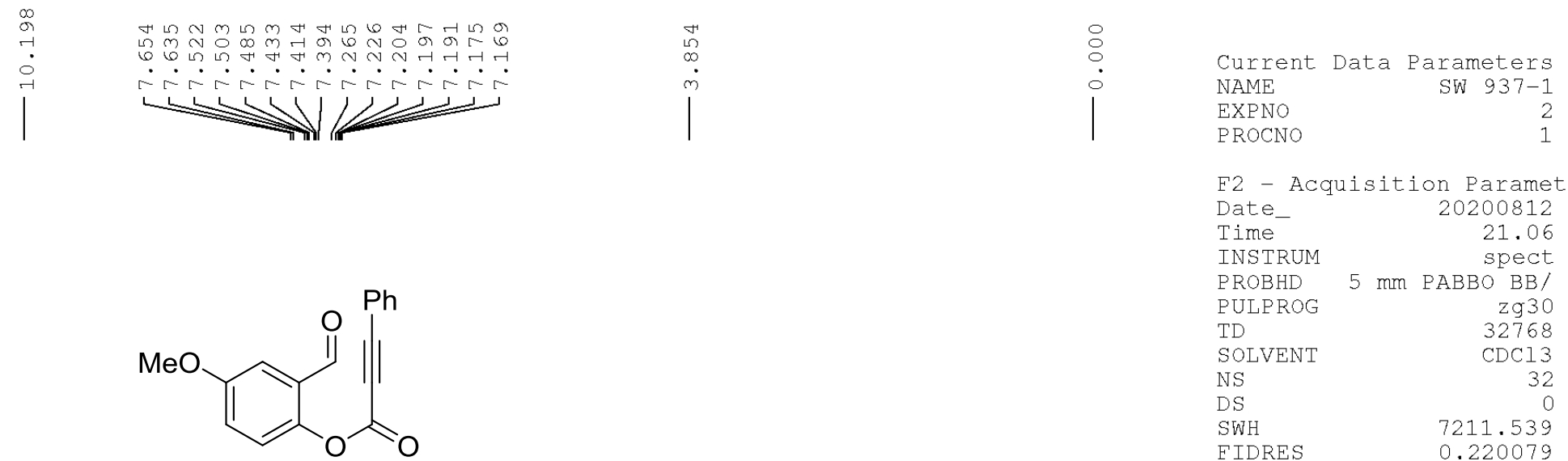
NAME
PROCNO

F2 - Acquisition Parameters

Date__ 20200812

$\begin{array}{lr}\text { Time } & 21.06\end{array}$

spect
INSTRUM $5 \mathrm{~mm} \mathrm{PABBO}$ BB/

PULPROG $\quad$ zg30

TD $\quad 32768$

SOLVENT CDCl3
32
NS

SWH $\quad 7211.539 \mathrm{~Hz}$

$\begin{array}{ll}7211.539 \mathrm{~Hz} \\ \text { FIDRES } & 0.220079 \mathrm{~Hz}\end{array}$

$\mathrm{AQ} \quad 2.2719147 \mathrm{sec}$

RG $\quad 57.42$

$\begin{array}{lr}\text { DW } & 69.333 \text { usec } \\ \text { DE } & 10.06 \text { usec }\end{array}$

TE $\quad 295.0 \mathrm{~K}$

D1 2.00000000 $\mathrm{sec}$

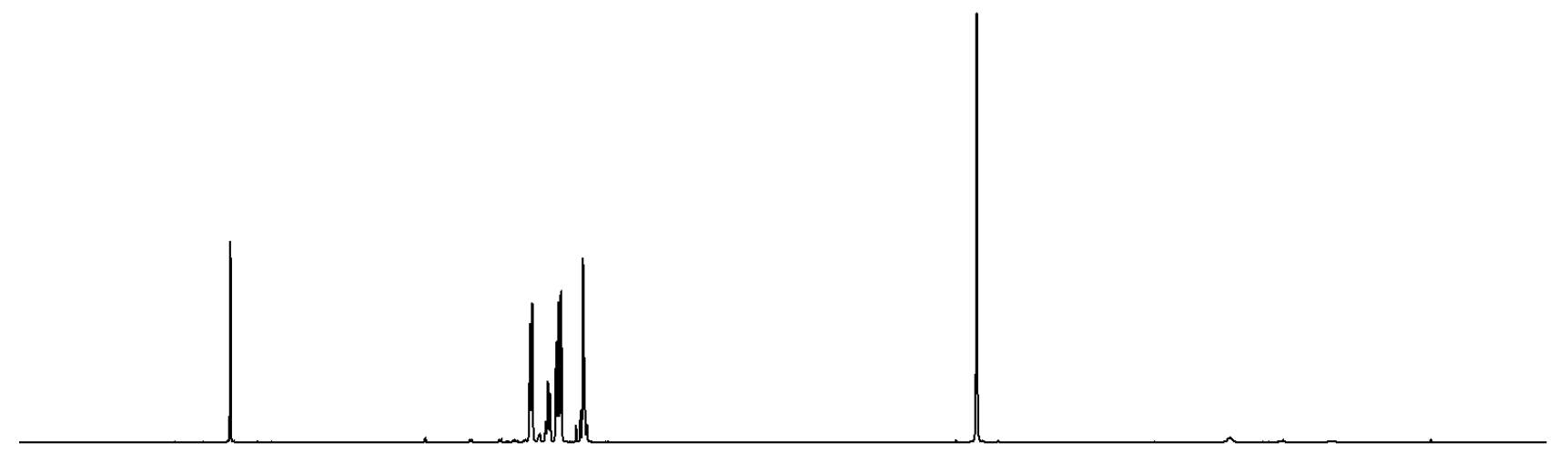

$=====$

CHANNEL $\mathrm{f} 1$

SEO1

$\mathrm{P} 1$

$400.1324008 \mathrm{MHz}$

P1

$1 \mathrm{H}$

2 - Processing parameter

SI $\quad 16384$

SF $\quad 400.1300074 \mathrm{MHz}$

WDW EM

SSB

LB

PC

1.00

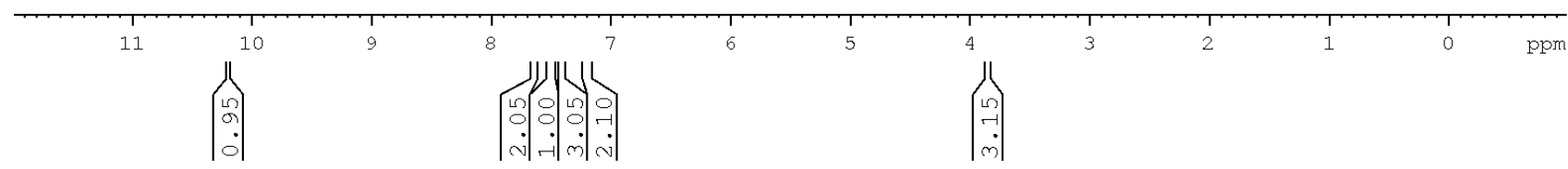


${ }^{13} \mathrm{C}$ NMR Spectrum of $\mathbf{9 c}\left(\mathrm{CDCl}_{3}, 100 \mathrm{MHz}\right)$

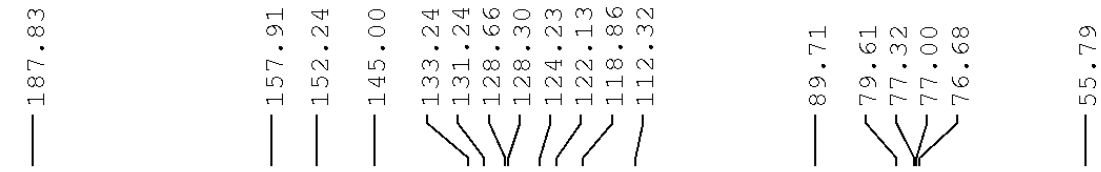

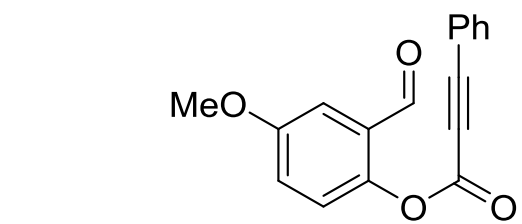

9c
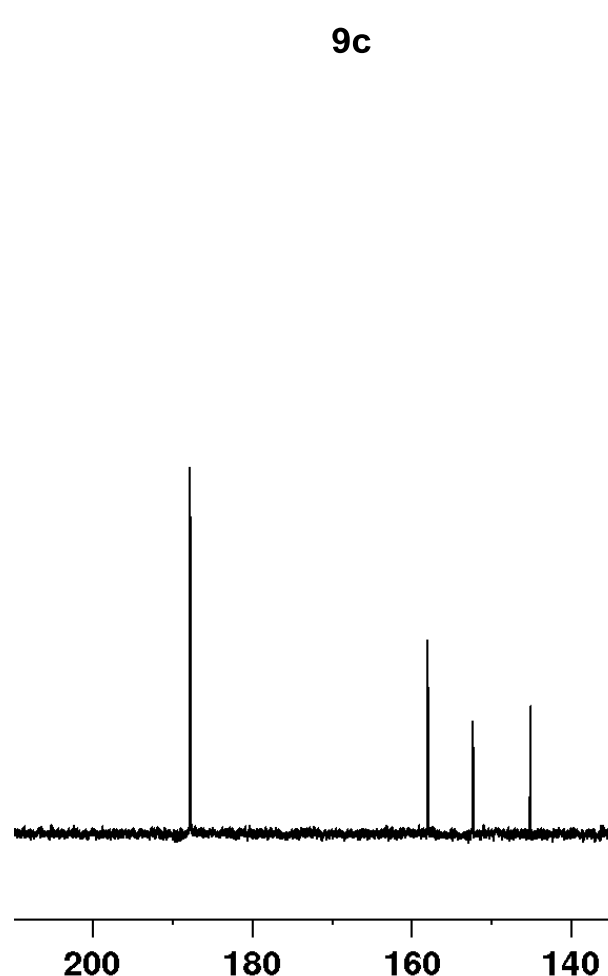

180

160

140

120

100

80

60

40

20

ppm

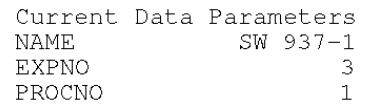

F2 - Acquisition Parameter

20200812

$\begin{array}{lr}\text { Time } & 21.11\end{array}$

PROBHD $5 \mathrm{~mm}$ PABBO BB/

PULPROG $\quad \mathrm{zgpg} 30$

$\begin{array}{ll}\text { TD } & 32768 \\ \text { SOLVENT } & \text { CDI3 }\end{array}$

NS

DS

SWH $24038.461 \mathrm{~Hz}$

FIDRES $\quad 0.733596 \mathrm{~Hz}$

AQ $\quad 0.6815744 \mathrm{sec}$

RG $\quad 198.09$

$\begin{array}{lr}\text { DW } & 20.800 \text { usec } \\ \text { DE } & 6.50 \text { usec }\end{array}$

$2.00000000 \mathrm{~K}$

D11 $\quad 0.03000000 \mathrm{sec}$

TDO

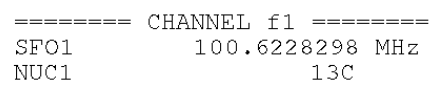

$\begin{array}{lr}\text { P1 } & 10.00 \text { usec } \\ \text { PLW1 } & 49.50000000 \mathrm{~W}\end{array}$

$\begin{array}{ll}======= & \text { CHANNEL } \mathrm{f} 2======== \\ \text { SFO2 } & 400.1316005 \mathrm{MHz}\end{array}$

$\mathrm{SFO} 2$

CPDPRG [2

PCPD2

PLW2

PLW12
PLW13 waltz16

90.00 usec

$12.50000000 \mathrm{~W}$

$0.34722000 \mathrm{~W}$

E2 - Processing parameters

SI 32768

SF $\quad 100.6127783 \mathrm{MH}$

WDW

$\mathrm{LB}$

$\mathrm{PC}$

$2.00 \mathrm{~Hz}$

1.00 
${ }^{1} \mathrm{H}$ NMR Spectrum of $\mathbf{1 2 a}\left(\mathrm{CDCl}_{3}, 400 \mathrm{MHz}\right)$

F2 - Acquisition Parameter

Date_ 20200903

Time 23.10

spect
INSTRUM $5 \mathrm{~mm} \mathrm{PABBO} \mathrm{BB} /$

PULPROG

TD $\quad 32768$

SOLVENT CDCl3

NS

DS $\quad 7211.539 \mathrm{~Hz}$

FIDRES $\quad 0.220079 \mathrm{~Hz}$

AQ $\quad 2.2719147 \mathrm{sec}$

RG $\quad 113.31$

DW $\quad 69.333$ usec

DE $\quad 10.06$ usec

TE

D1

$2.00000000 \mathrm{sec}$

$====$

SFO1

NUC

CHANNEL $\mathrm{f} 1=======$

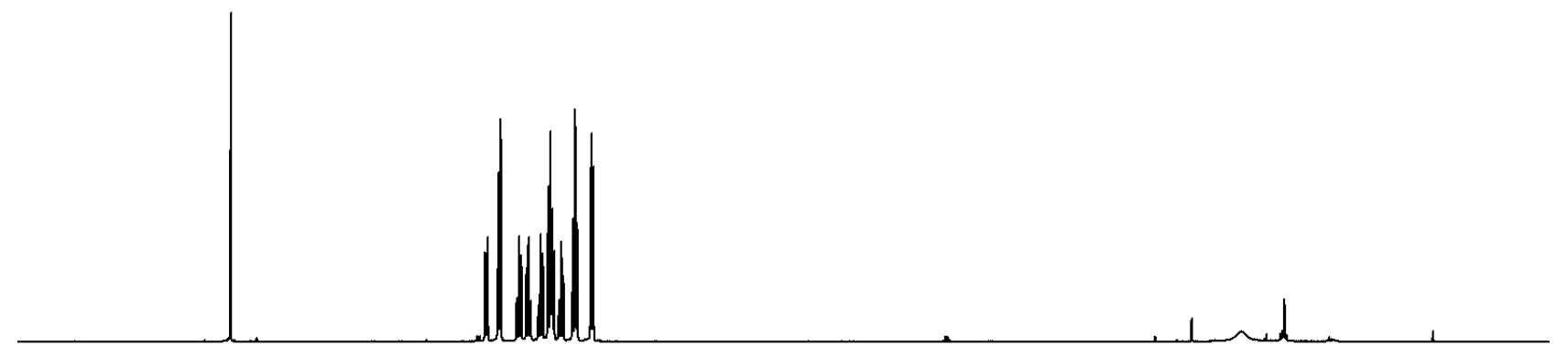

PLW1

$400.1324008 \mathrm{MHz}$ 15.00

11.39999962 w

F2 - Processing parameters

SI 16384

SF $\quad 400.1300101 \mathrm{MHz}$

WDW

SSB
LB

GB

$\mathrm{PC}$

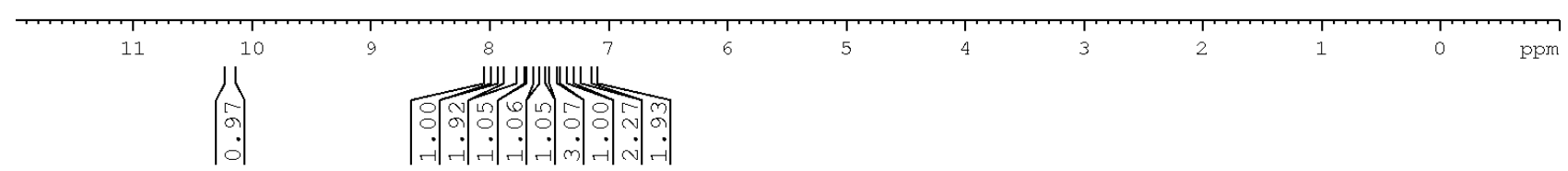


${ }^{13} \mathrm{C}$ NMR Spectrum of $\mathbf{1 2 a}\left(\mathrm{CDCl}_{3}, 100 \mathrm{MHz}\right)$
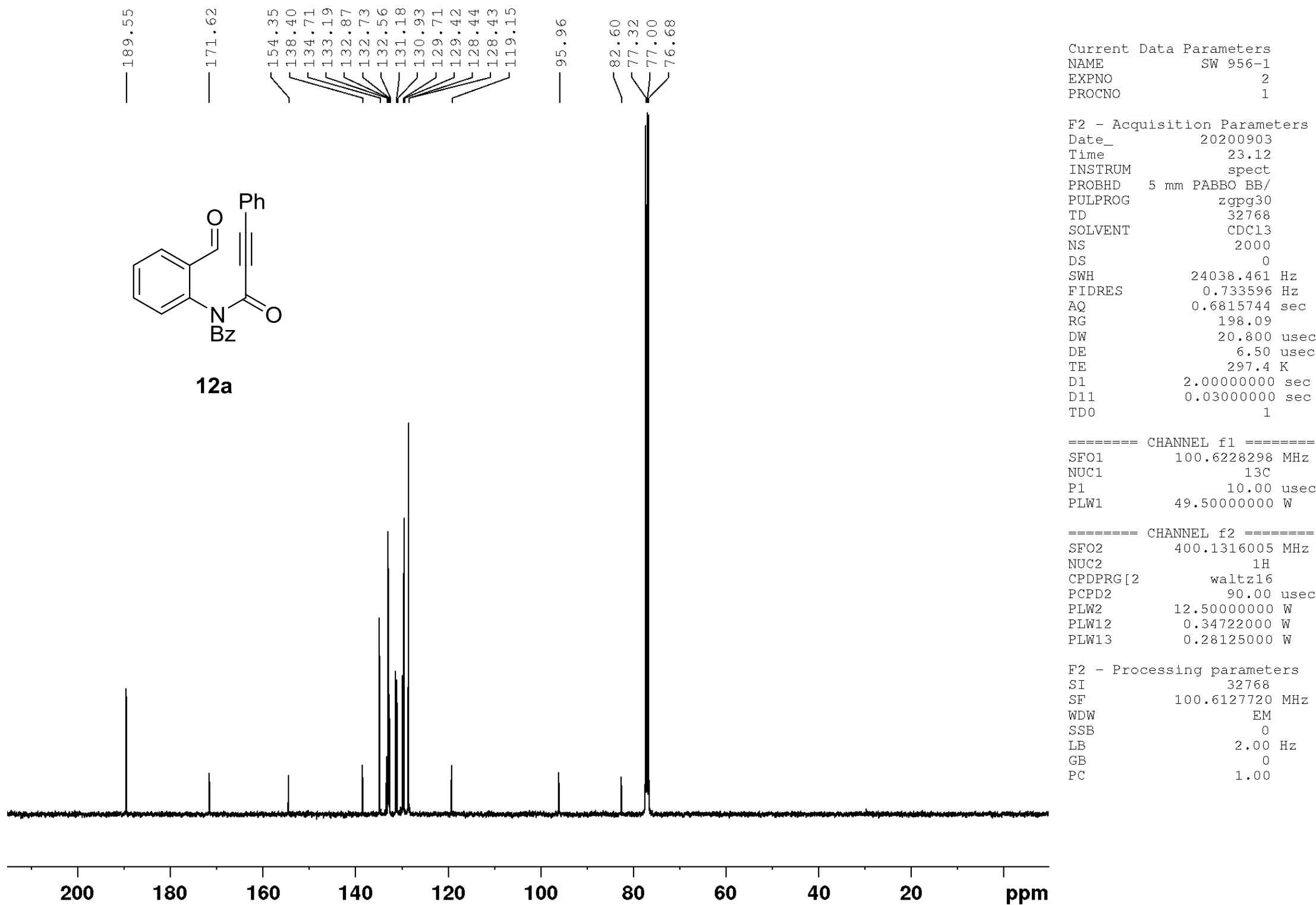
${ }^{1} \mathrm{H}$ NMR Spectrum of $\mathbf{4 a a}\left(\mathrm{CDCl}_{3}, 400 \mathrm{MHz}\right)$

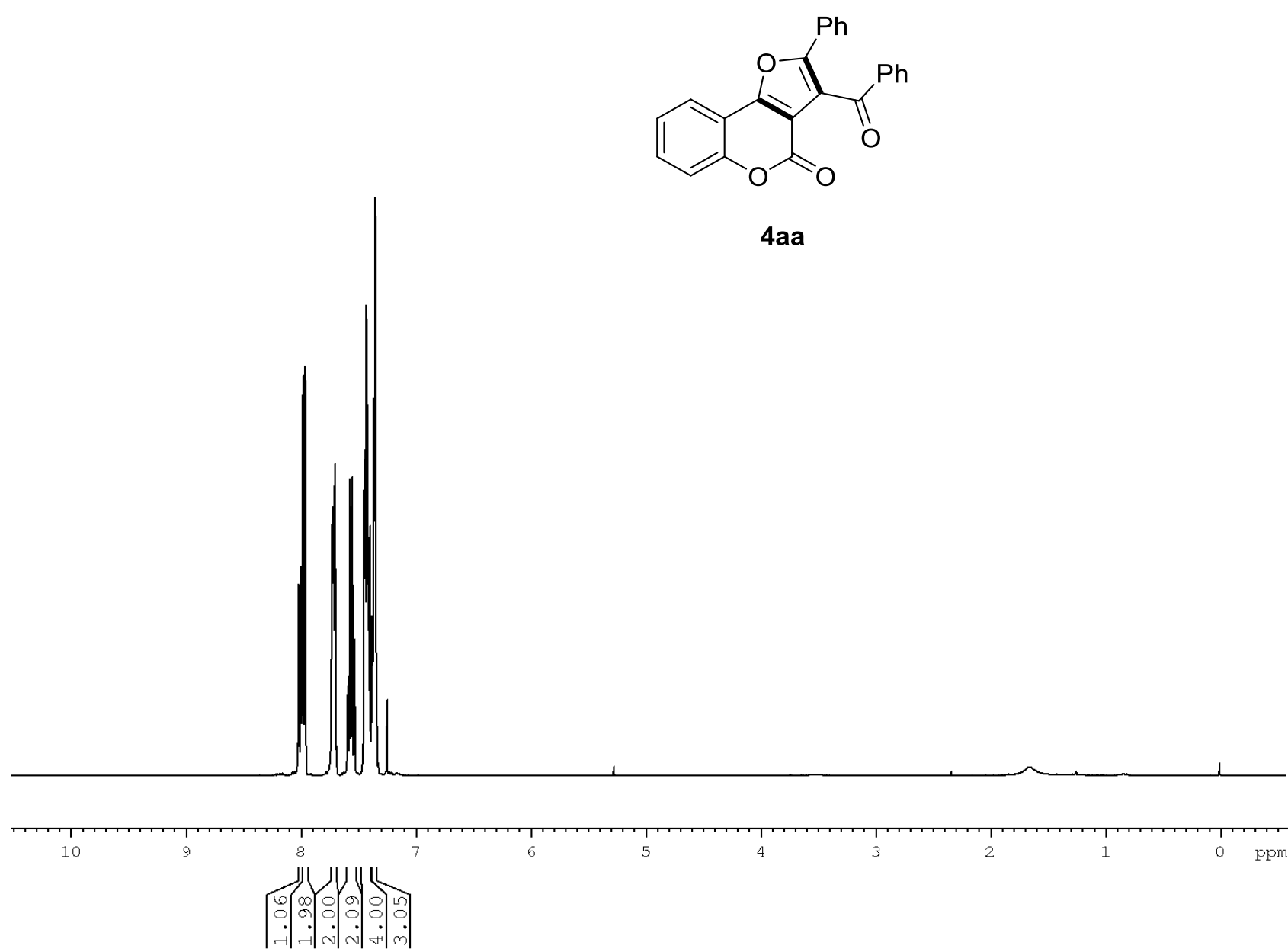


${ }^{13} \mathrm{C}$ NMR Spectrum of $4 a a\left(\mathrm{CDCl}_{3}, 100 \mathrm{MHz}\right)$

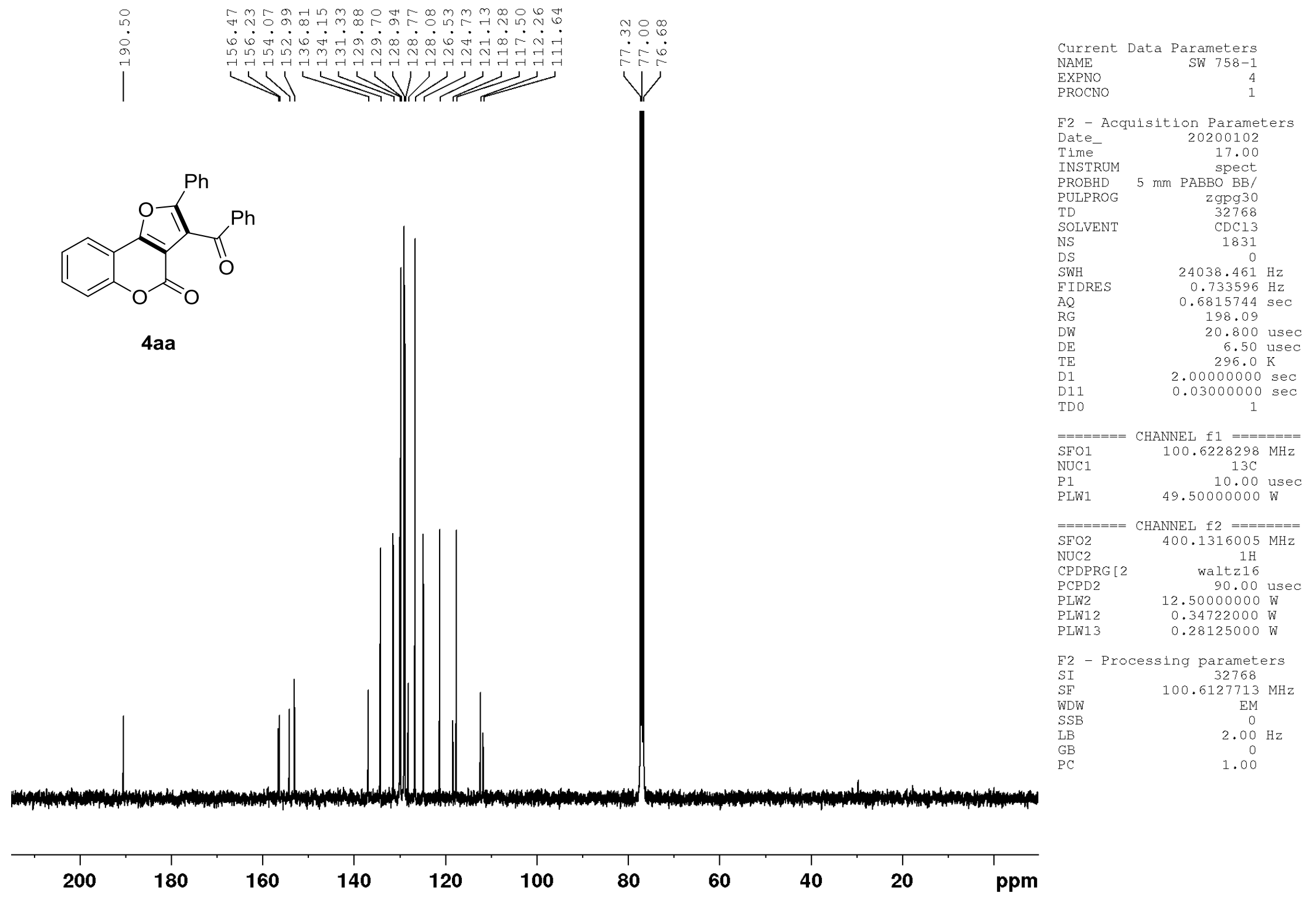


${ }^{1} \mathrm{H}$ NMR Spectrum of $\mathbf{4 b a}\left(\mathrm{CDCl}_{3}, 400 \mathrm{MHz}\right)$

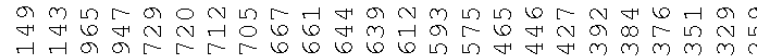

$7 \mathrm{H}$

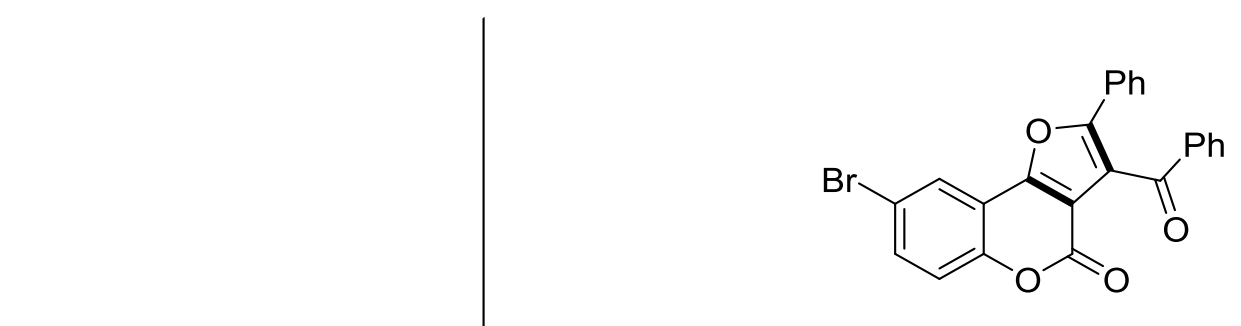

$4 b a$

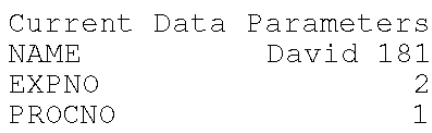

EXPNO

F2 - Acquisition Parameters

Date_ 20200723

Time 22.01

INSTRUM spect

$5 \mathrm{~mm}$ BBO $\mathrm{BB}-1 \mathrm{H}$

PUT

zg30

SOLVENT

NS

DS

$\mathrm{SWH}$

FIDRES

$\mathrm{AQ}$
$\mathrm{RG}$

RG

DW

DE

D1

TDO

CDCl 3

32
0

$7246.377 \mathrm{~Hz}$

$0.221142 \mathrm{~Hz}$ $2.2609921 \mathrm{sec}$

$$
362
$$

69.000 usec

6.50 usec

$297.5 \mathrm{~K}$

$2.00000000 \mathrm{sec}$

$\begin{array}{lr}======\text { CHANNEL } \mathrm{f} 1======= \\ \text { NUC1 } & 1 \mathrm{H} \\ \text { P1 } & 15.00 \mathrm{usec} \\ \text { PL1 } & 11.10 \mathrm{~dB} \\ \text { SE01 } & 1324008 \mathrm{MHz}\end{array}$

SFO1 $400.1324008 \mathrm{MHz}$

F2 - Processing parameters

SI Processing parameters 16384

SF $\quad 400.1300113 \mathrm{MHz}$

WDW

SSB

$\mathrm{HB}$

GB

OM
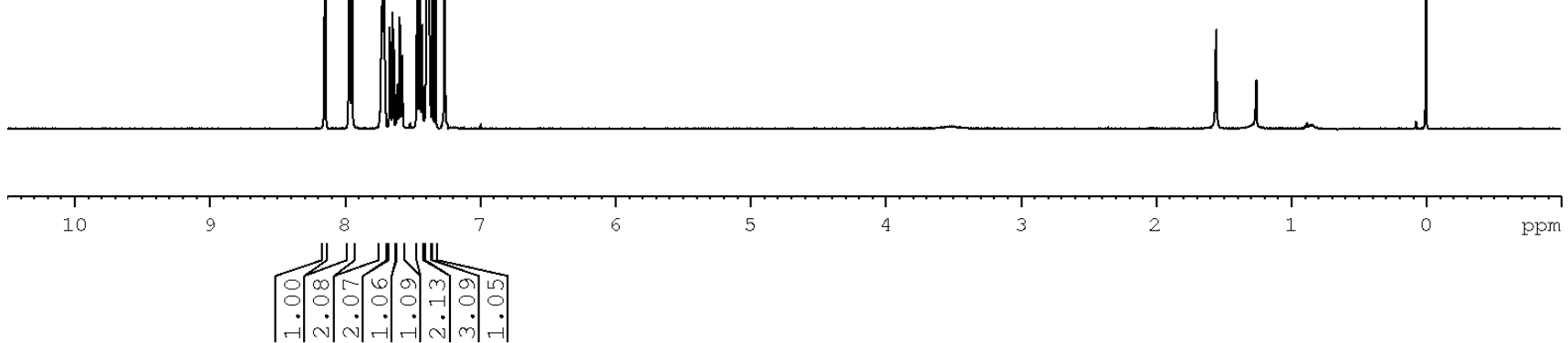
${ }^{13} \mathrm{C}$ NMR Spectrum of $4 \mathbf{b a}\left(\mathrm{CDCl}_{3}, 100 \mathrm{MHz}\right)$
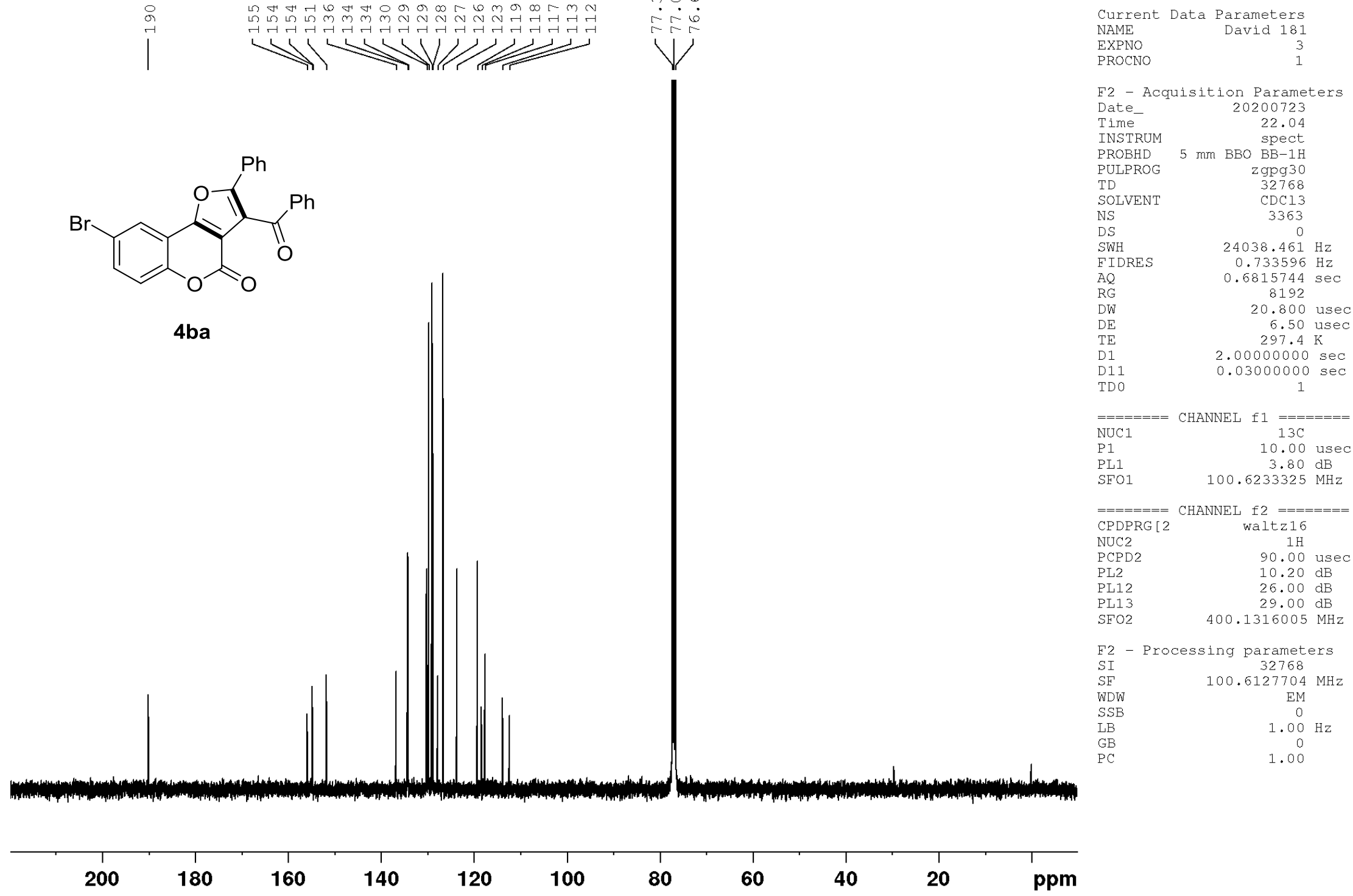
${ }^{1} \mathrm{H}$ NMR Spectrum of $4 \mathbf{c a}\left(\mathrm{CDCl}_{3}, 400 \mathrm{MHz}\right)$

$\begin{array}{lll}0 & \\ 0 & 0\end{array}$

$\rightarrow 1+1$

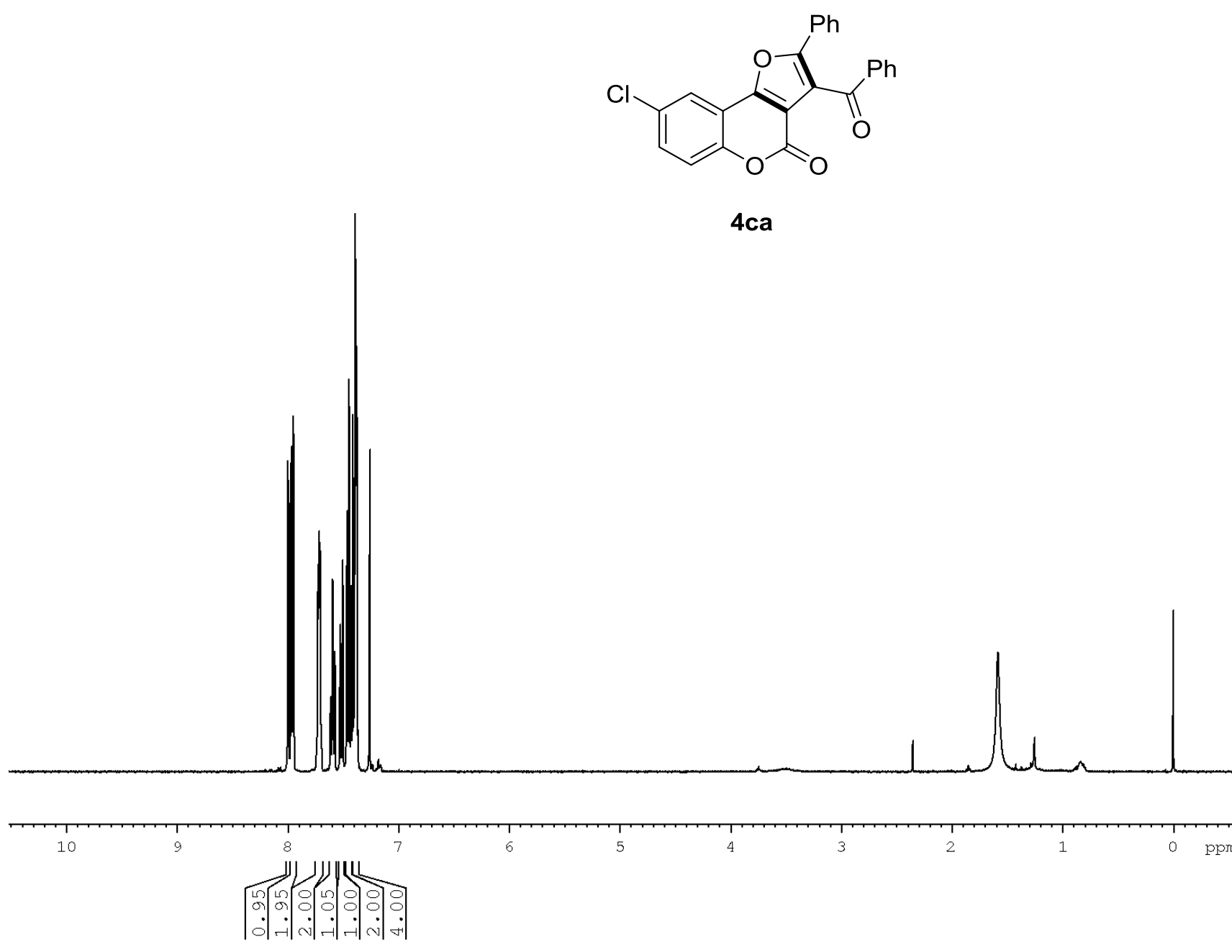

$\begin{array}{lr}\text { Current Data Parameters } \\ \text { NAME } & \text { Carol } 001 \\ \text { EXPNO } & 1 \\ \text { PROCNO } & 1\end{array}$

F2 - Acquisition Parameters

Date__ 20200803

Time 20.09

INSTRUM spect

PROBHD $5 \mathrm{~mm}$ BBO $\mathrm{BB}-1 \mathrm{H}$

PULPROG $\quad$ zg30

SWH

FIDRES

$\mathrm{AQ}$

RG

$D W$

DE

D1

DO

NUC1

$\mathrm{zg} 30$
32768

$\mathrm{CDCl} 3$

32
0

$7246.377 \mathrm{~Hz}$

$0.221142 \mathrm{~Hz}$

$2.2609921 \mathrm{sec}$

256

69.000 usec

6.50 usec

$2.00000000 \mathrm{sec}$

$\mathrm{P} 1$

PLI

CHANNEL $f$

2 - Processing parameters

SI 16384

SF $\quad 400.1300110 \mathrm{MHz}$

WDW

SSB

LB

GB

$\mathrm{EM}$

1.00 
${ }^{13} \mathrm{C}$ NMR Spectrum of $4 \mathbf{c a}\left(\mathrm{CDCl}_{3}, 100 \mathrm{MHz}\right)$
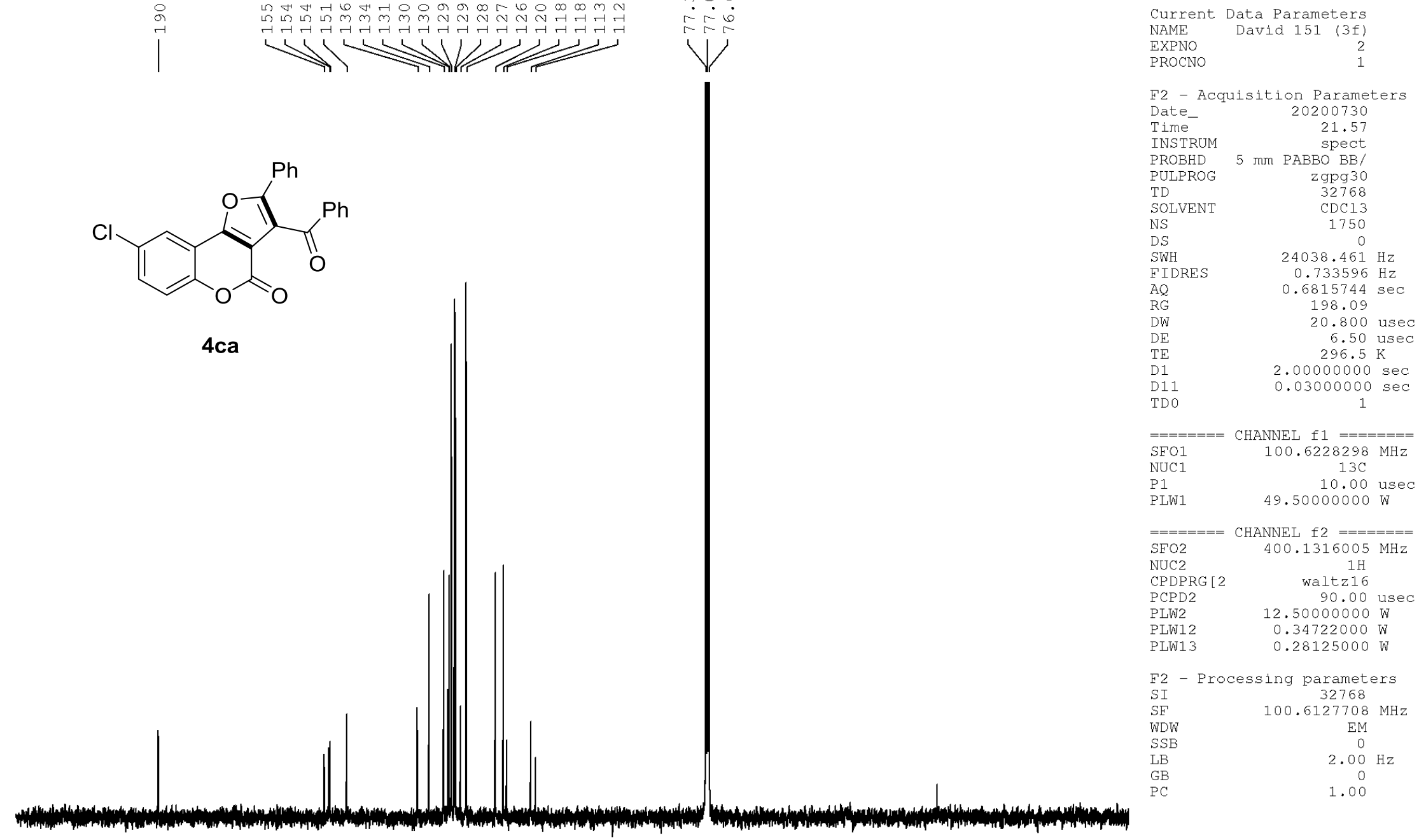
${ }^{1} \mathrm{H}$ NMR Spectrum of $\mathbf{4 d a}\left(\mathrm{CDCl}_{3}, 400 \mathrm{MHz}\right)$

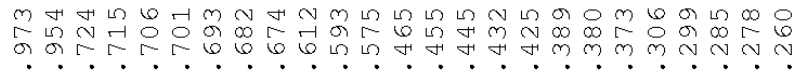

$\rightarrow$
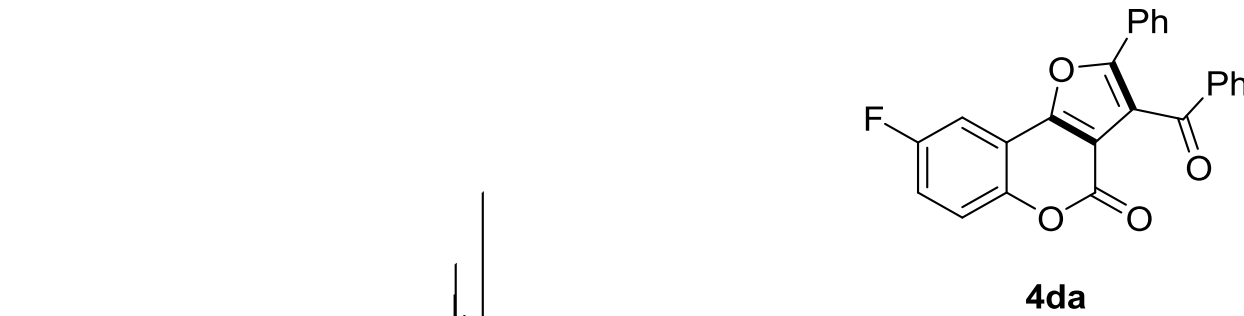

Current Data Parameters

NAME David 170 (3f)

PROCNO

F2 - Acquisition Parameters

Date_ 20200731

Time

16.52

INSTRUM

PROBHD

mm $\mathrm{PABBO} \mathrm{BB} /$

$\mathrm{TD}$

SOLVENT

NS

DS

SWH

FIDRES

$\mathrm{AQ}$
$\mathrm{RG}$
$\mathrm{DW}$

$\mathrm{DE}$

TDO

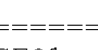

$\mathrm{SEO}$

NUC1

P1

$\mathrm{Zg} 30$
32768

32

0
$7211.539 \mathrm{~Hz}$

$0.220079 \mathrm{~Hz}$

$2.2719147 \mathrm{sec}$

113.31

69.333 usec

10.06 usec

$293.7 \mathrm{~K}$

$2.00000000 \mathrm{sec}$

CHANNEI f1 1

LW 1

$400.1324008 \mathrm{MHz}$ $1 \mathrm{H}$

15.00 used

$11.39999962 \mathrm{~W}$

F2 - Processing parameters

SI 16384

SF $\quad 400.1300096 \mathrm{MHz}$

$[\mathrm{WDN}$

SSB

LB

GB

$\mathrm{EM}$
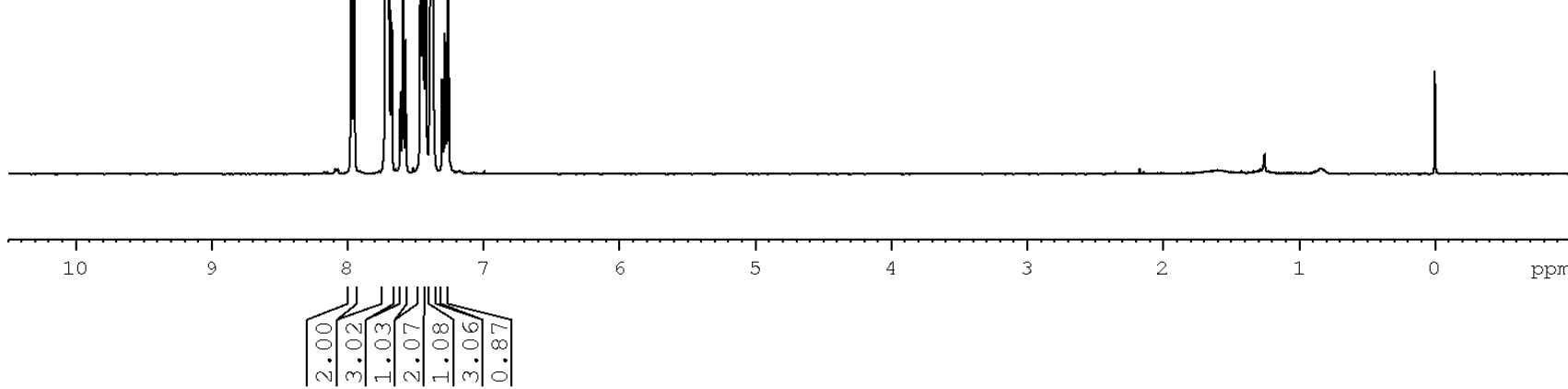
${ }^{13} \mathrm{C}$ NMR Spectrum of $4 \mathrm{da}\left(\mathrm{CDCl}_{3}, 100 \mathrm{MHz}\right)$

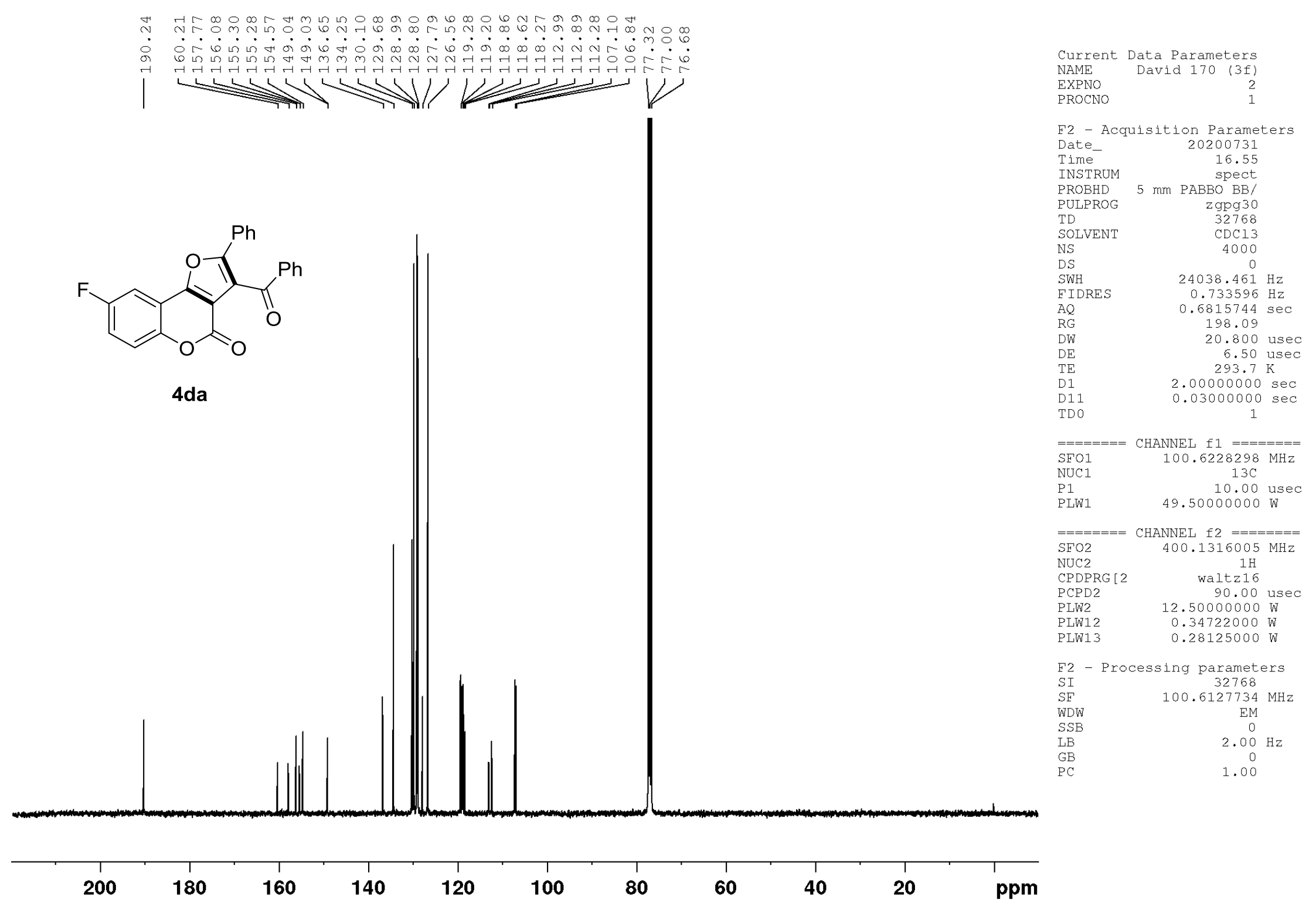


${ }^{19} \mathrm{~F}$ NMR Spectrum of $4 \mathbf{d a}\left(\mathrm{CDCl}_{3}, 376 \mathrm{MHz}\right)$

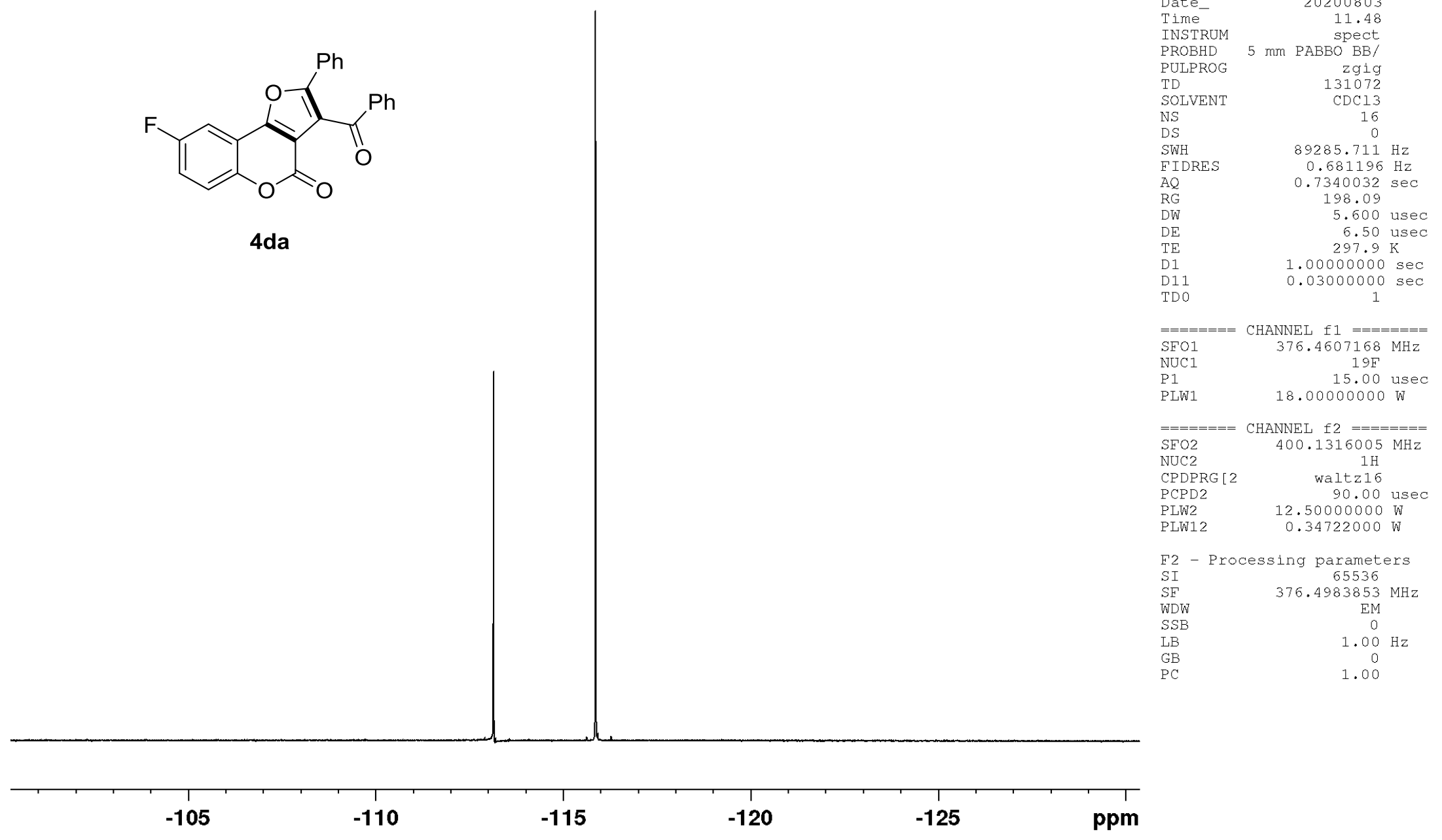


${ }^{1} \mathrm{H}$ NMR Spectrum of $4 \mathrm{ea}\left(\mathrm{CDCl}_{3}, 400 \mathrm{MHz}\right)$
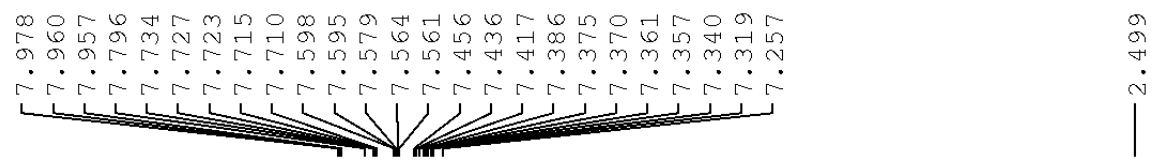

$\begin{array}{lrr}\text { Current } & \text { Data Parameters } \\ \text { NAME } & \text { David } 189 & (3 \mathrm{f}) \\ \text { EXPNO } & & 1 \\ \text { PROCNO } & & 1\end{array}$

F2 - Acquisition Parameters

Date 20200814

Time 21.55

INSTRUM spect

PROBHD $5 \mathrm{~mm}$ PABBO BB/<smiles>Cc1ccc2oc(=O)c3c(C(=O)c4ccccc4)c(-c4ccccc4)oc3c2c1</smiles>

PULPROG $\quad$ zg30

TD 32768

$\begin{array}{lr}\text { SOLVENT } & 32 \\ \text { NS } & \end{array}$

DS

SWH $\quad 7211.539 \mathrm{~Hz}$

FIDRES $\quad 0.220079 \mathrm{~Hz}$

AQ $\quad 2.2719147 \mathrm{sec}$

RG $\quad 99.72$

DW $\quad 69.333$ usec

$\mathrm{DE} \quad 10.06$ usec

TE $296.2 \mathrm{~K}$

$\begin{array}{lr}\text { D1 } & 2.00000000 \text { sec } \\ \text { TDO } & 1\end{array}$

4ea

$====$

$\mathrm{SFO} 1$

P1

PLW1

CHANNEL $\mathrm{f} 1$

$$
1
$$

F2 - Processing parameters

$\begin{array}{cc}\mathrm{F} 2 & - \text { Processing parameter } \\ \mathrm{SI} & 16384\end{array}$

SF $\quad 400.1300109 \mathrm{MHz}$

WDW

SSB

LB

$G B$
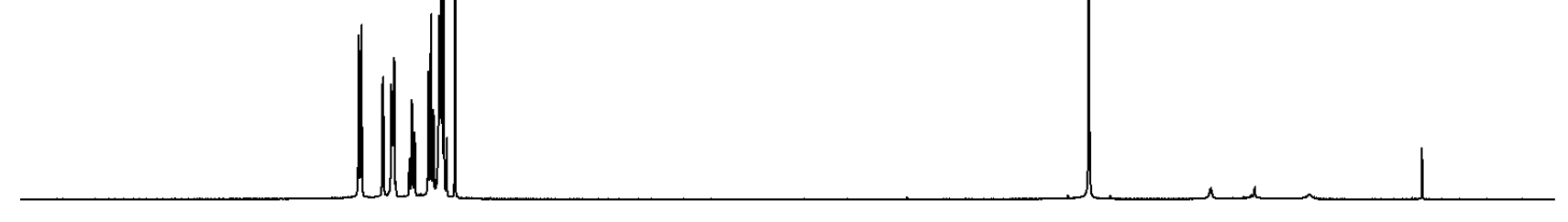

1.00

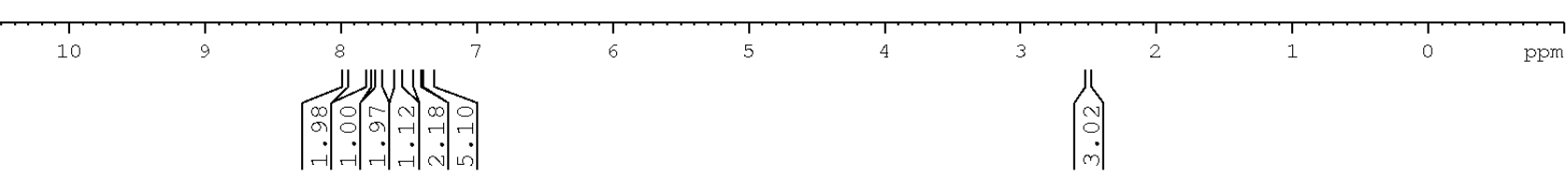


${ }^{13} \mathrm{C}$ NMR Spectrum of 4 ea $\left(\mathrm{CDCl}_{3}, 100 \mathrm{MHz}\right)$

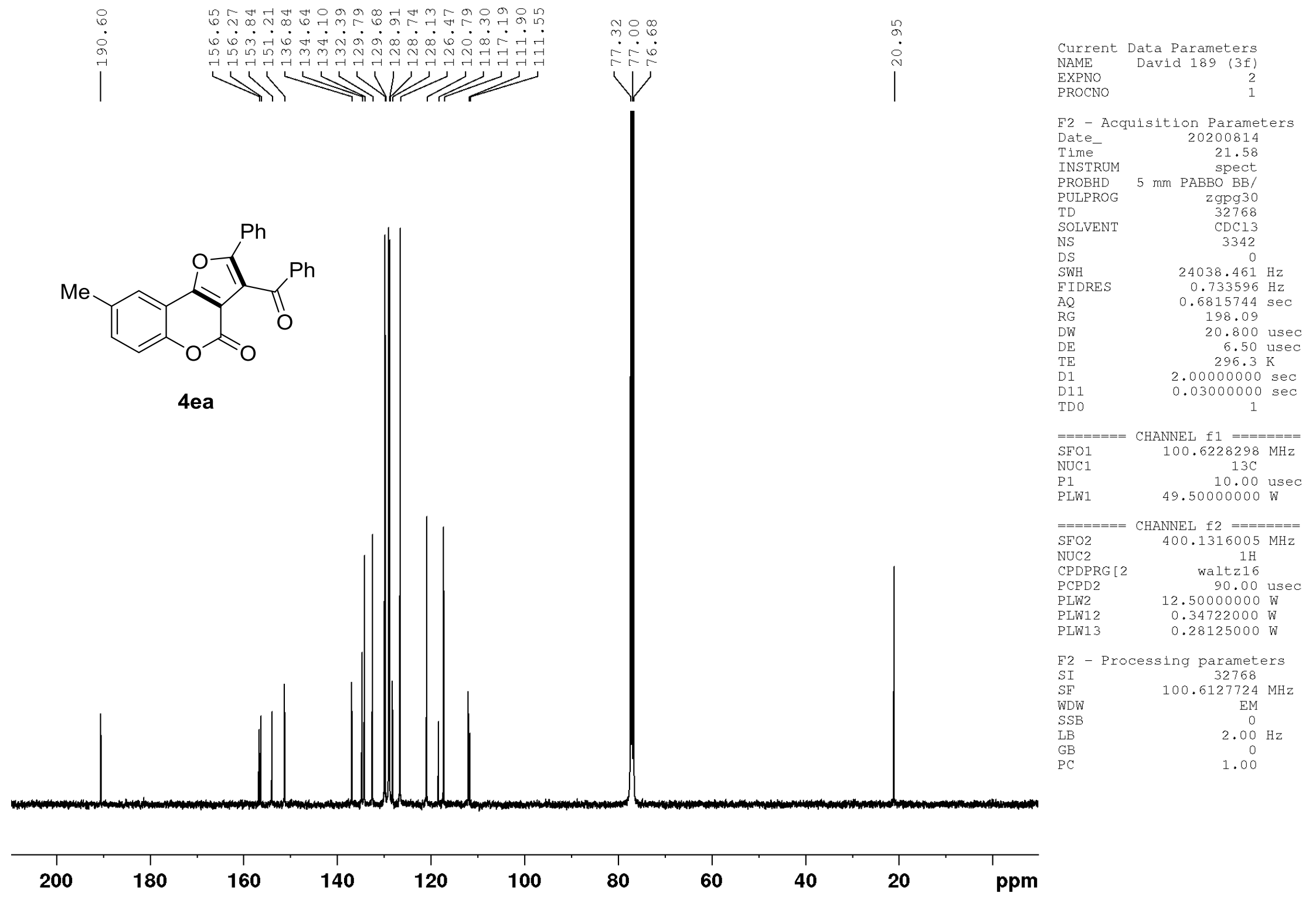


$\underline{{ }^{1} \mathrm{H} N M R \text { Spectrum of } \mathbf{4 f a}\left(\mathrm{CDCl}_{3}, 400 \mathrm{MHz}\right)}$
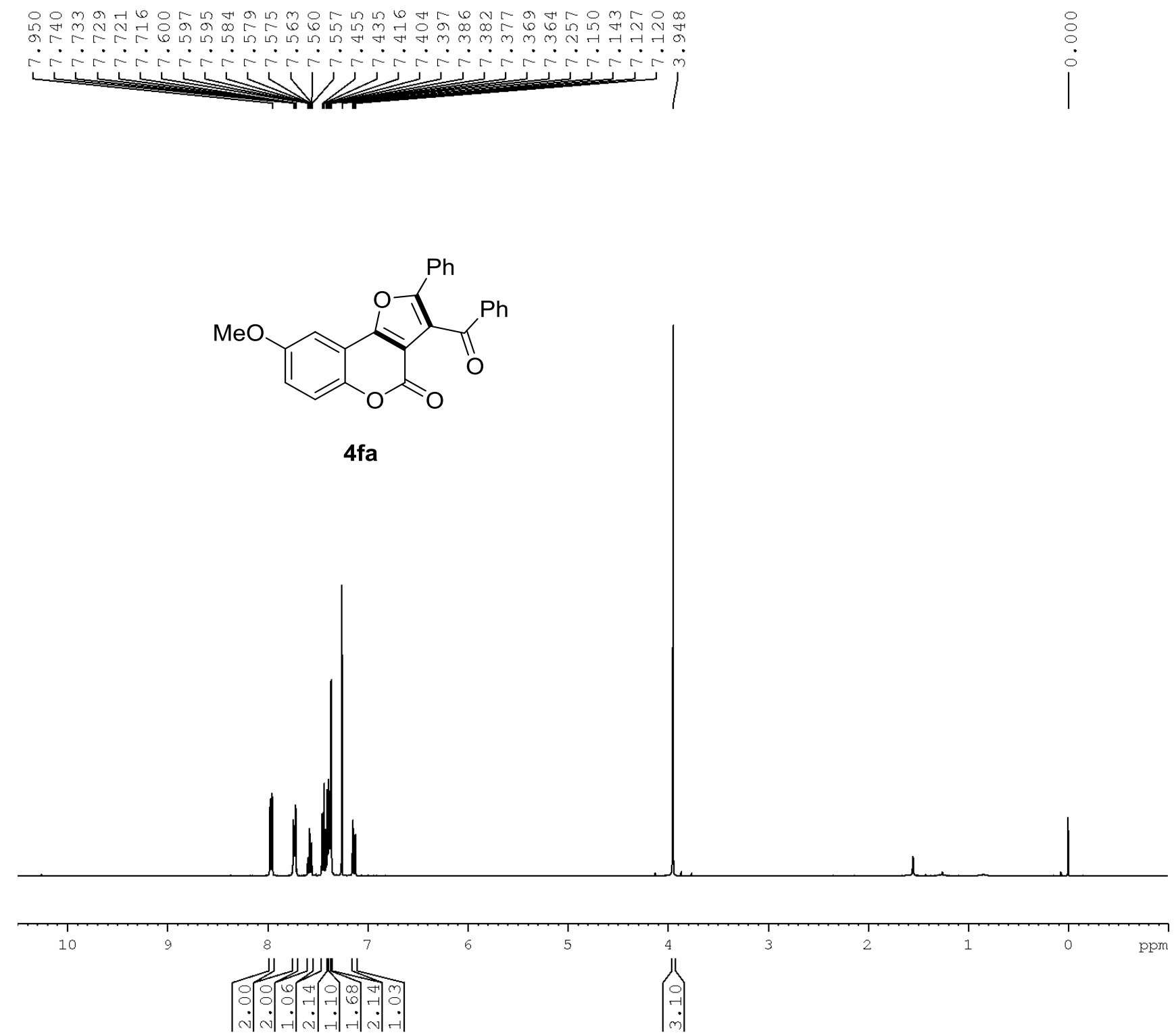

$\begin{array}{lrr}\text { Current } & \text { Data Parameters } \\ \text { NAME } & \text { Carol } 006 & (3 \mathrm{f}) \\ \text { EXPNO } & & 1 \\ \text { PROCNO } & & 1\end{array}$

F2 - Acquisition Parameters

$\begin{array}{lr}\text { Date_ } & 20200718 \\ \text { Time } & 22.33\end{array}$

Time

INSTRUM

PROBHD

spect

PULPROG

SOLVENT

NS

DS

SWH

FIDRES

$\mathrm{AQ}$

DW

$\mathrm{DE}$

D1

TDO

$====$

SEO

NUC

$\mathrm{mm}$ PABBO BB/
$\mathrm{zg} 30$

$\mathrm{zg} 30$
32768

$\mathrm{CDCl} 3$

0
$7211.539 \mathrm{~Hz}$

$0.220079 \mathrm{~Hz}$

$2.2719147 \mathrm{sec}$

198.09

69.333 usec

10.06 usec

sec

PLW1

HANNEL $\mathrm{fl}=======$ $400.1324008 \mathrm{MHz}$ $1 \mathrm{H}$ 5.00 usec

F2 - Processing parameters

SI 16384

SF $\quad 400.1300106 \mathrm{MHz}$

WDW

SSB
LB

$\mathrm{LB}$
$\mathrm{GB}$

$\mathrm{PC}$

400.1300106

EM
0 
${ }^{13} \mathrm{C}$ NMR Spectrum of $\mathbf{4 f a}\left(\mathrm{CDCl}_{3}, 100 \mathrm{MHz}\right)$

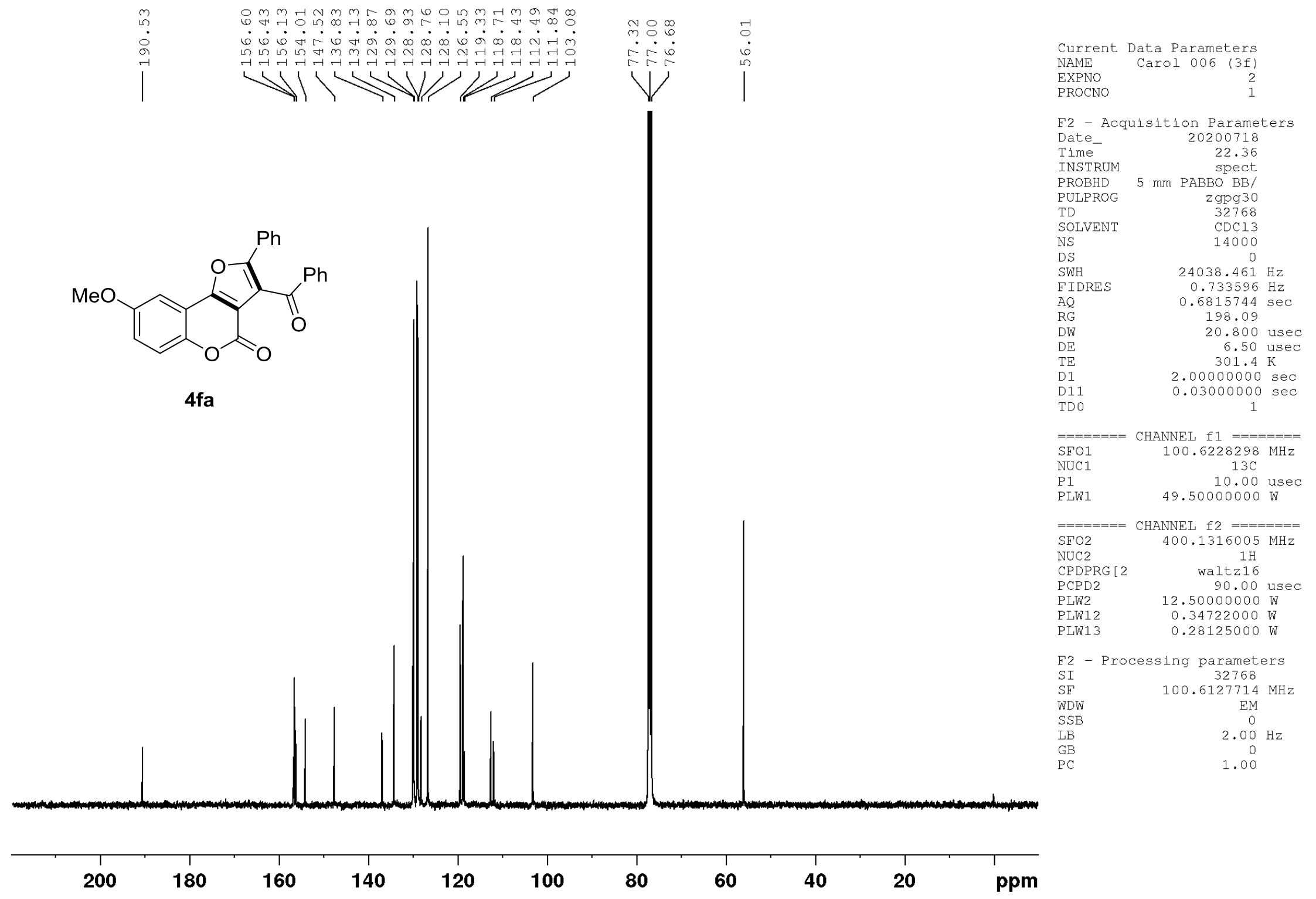


$\underline{{ }^{1} \mathrm{H} \text { NMR Spectrum of } 4 \mathrm{ga}\left(\mathrm{CDCl}_{3}, 400 \mathrm{MHz}\right)}$

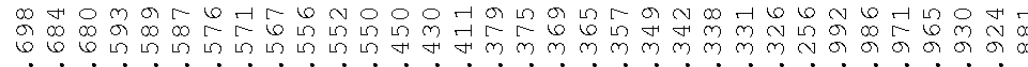
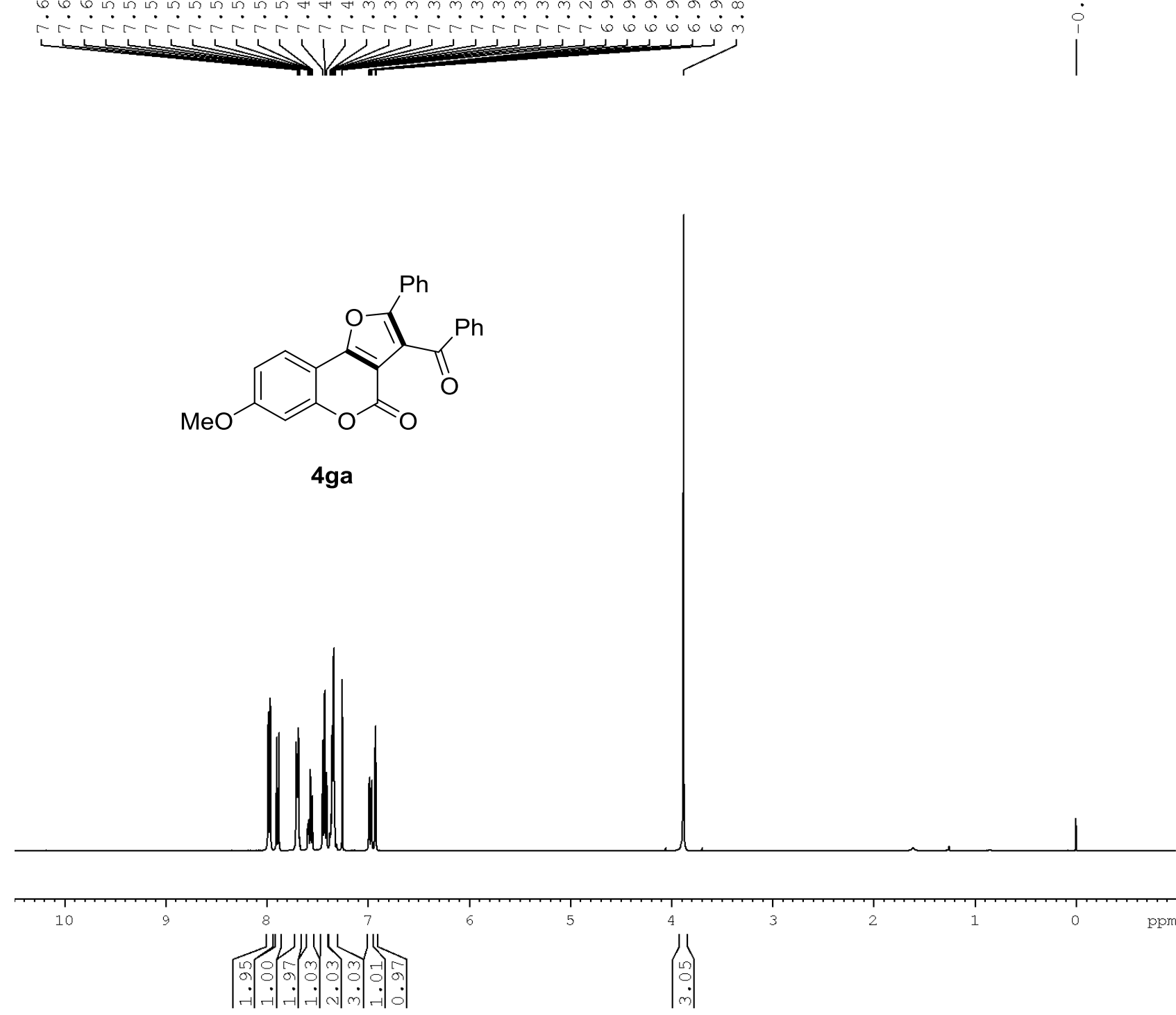

$\begin{array}{lrr}\text { Current } & \text { Data Parameters } \\ \text { NAME } & \text { Carol } 007 & (3 \mathrm{f}) \\ \text { EXPNO } & & 1 \\ \text { PROCNO } & & 1\end{array}$

F2 - Acquisition Parameters

Date_ 20200722

Time 22.08

INSIRUM spect

PROBHD $5 \mathrm{~mm}$ PABBO BB/

PULPROG $\quad$ zg30

TD 32768

$\mathrm{CDCl} 32$

SWH $\quad 7211.539 \mathrm{~Hz}$

TIDRFS $\quad 7211.539 \mathrm{~Hz}$

$0.220079 \mathrm{~Hz}$

$\begin{array}{lr}\text { AQ } & 2.2719147 \mathrm{sec} \\ \text { RG } & 78.51\end{array}$

DW $\quad 69.333$ usec

$\mathrm{DE} \quad 10.06$ use

$298.2 \mathrm{~K}$

D1 $\quad 2.00000000 \mathrm{sec}$

$===$

SFO1

NUC1

P1

CHANNEL $\mathrm{f} 1========$

15.00 usec

11.39999962

F2 - Processing parameters

SI 16384

SF $\quad 400.1300110 \mathrm{MHz}$

WDW

SSB
LB

GB

$\mathrm{PC}$ 
${ }^{13} \mathrm{C}$ NMR Spectrum of $4 \mathrm{ga}\left(\mathrm{CDCl}_{3}, 100 \mathrm{MHz}\right)$

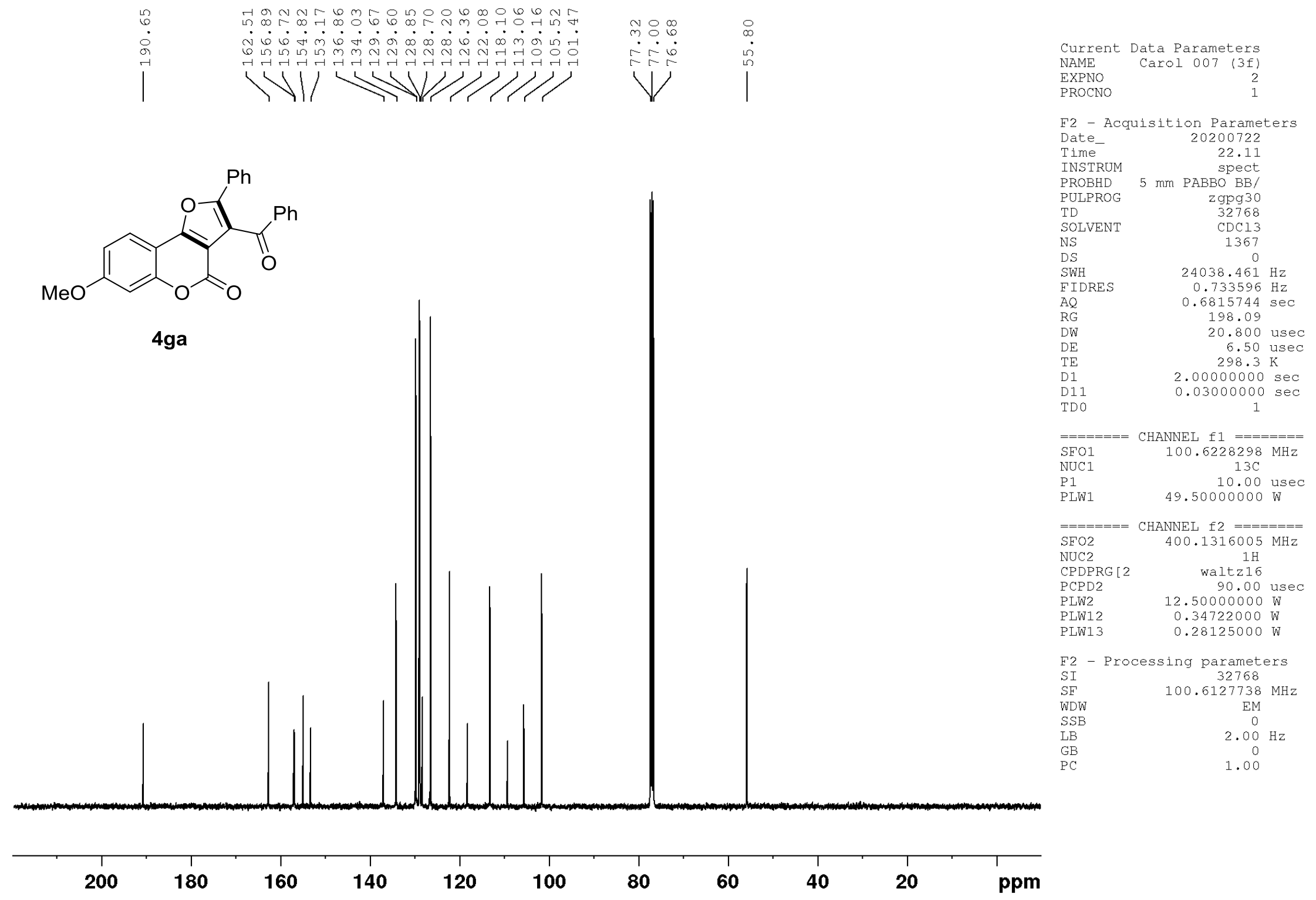


${ }^{1} \mathrm{H}$ NMR Spectrum of 4 ha $\left(\mathrm{CDCl}_{3}, 400 \mathrm{MHz}\right)$
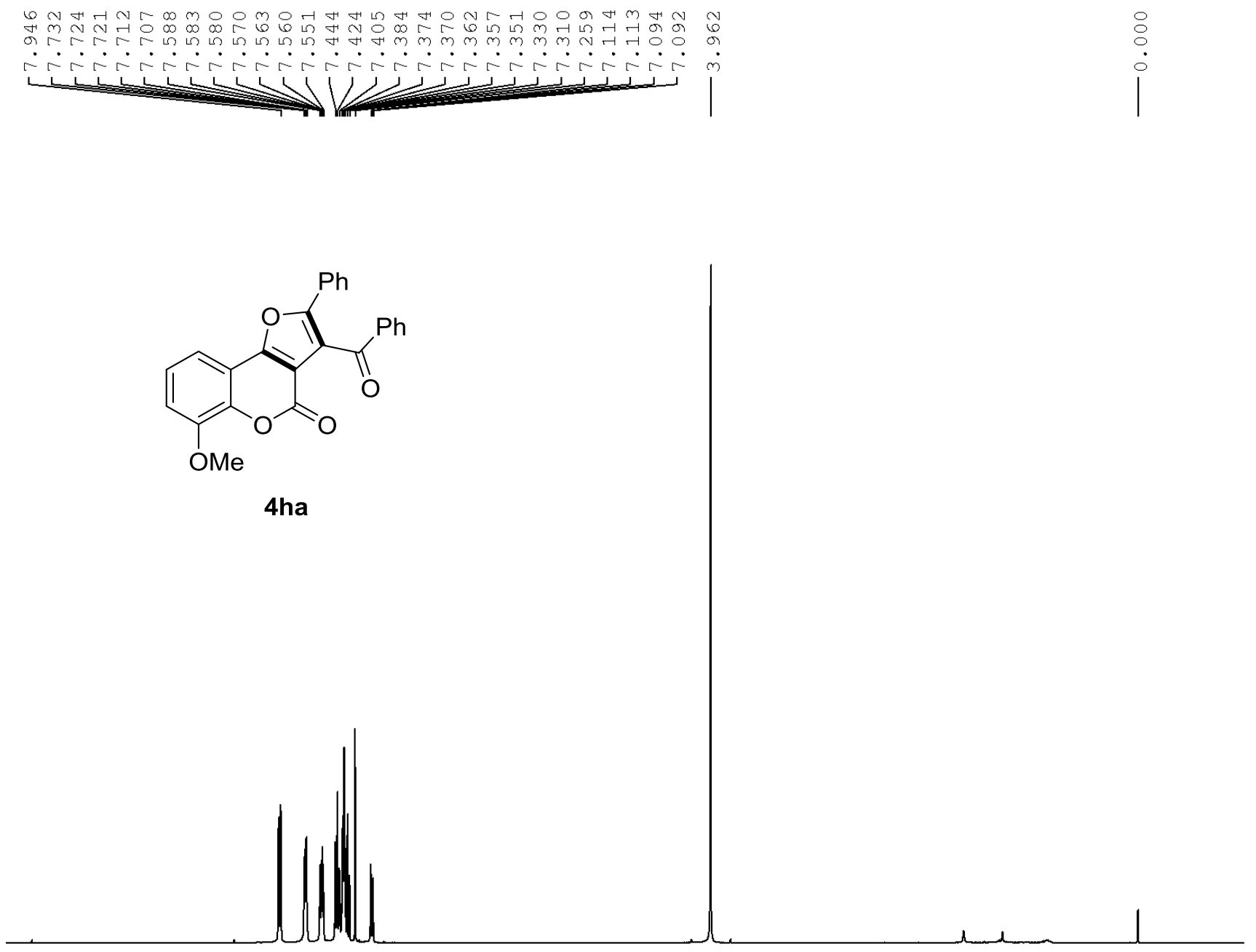

$\begin{array}{lrr}\text { Current Data Parameters } \\ \text { NAME } & \text { Carol } 008 & (3 \mathrm{f}) \\ \text { EXPNO } & & 1 \\ \text { PROCNO } & & 1\end{array}$

F2 - Acquisition Parameters

Date_

20200731

ime

INSTRU

ROBHD

PULPROG

SOLVENT

NS

DS

SWH

FIDRES

AQ

DW

DE

D1

TDO

$=====$

$\mathrm{SEO}$

NUC

P1

21.53

$5 \mathrm{~mm}$ spect

$\mathrm{BB} /$

$\mathrm{zg} 30$
32768

32

0
$7211.539 \mathrm{~Hz}$
$0.220079 \mathrm{~Hz}$

$0.220079 \mathrm{~Hz}$

$2.2719147 \mathrm{sec}$

99.72

69.333 usec

10.06 usec

.00000

sec

CHANNEL $\mathrm{f} 1========$ $400.1324008 \mathrm{MHz}$
(n)

15.00 use
11.39999962 .

F2 - Processing parameters

SI 16384

SF $\quad 400.1300103 \mathrm{MHz}$

WDW

SSB

LB

$\mathrm{PC}$

0

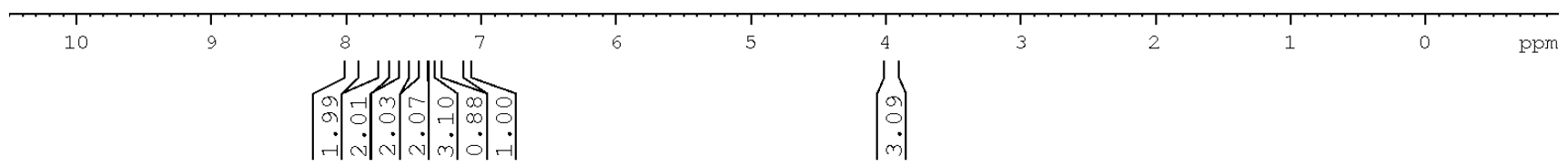


${ }^{13} \mathrm{C}$ NMR Spectrum of 4 ha $\left(\mathrm{CDCl}_{3}, 100 \mathrm{MHz}\right)$
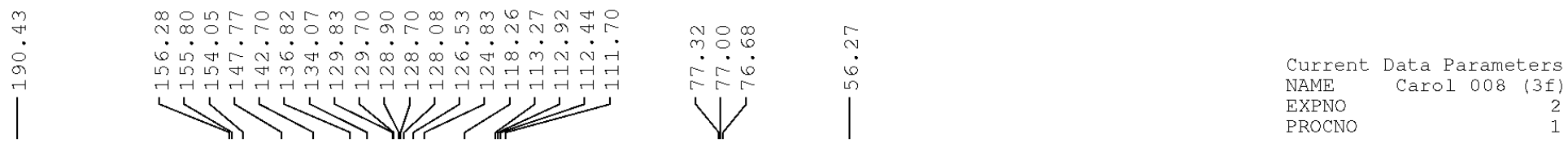

F2 - Acquisition Parameter

Date__ 20200731

Time 21.55
spect

PROBHD $5 \mathrm{~mm}$ PABBO BB/

PULPROG zgpg30<smiles>COc1cccc2c1oc(=O)c1c(C(=O)c3ccccc3)c(-c3ccccc3)oc12</smiles>

TD

DS

SWH

$24038.461 \mathrm{~Hz}$

$0.733596 \mathrm{~Hz}$

4ha

0.6815744 sec

198.09

20.800 usec

6.50 usec
$296.5 \mathrm{~K}$

$2.00000000 \mathrm{sec}$

$0.03000000 \mathrm{sec}$

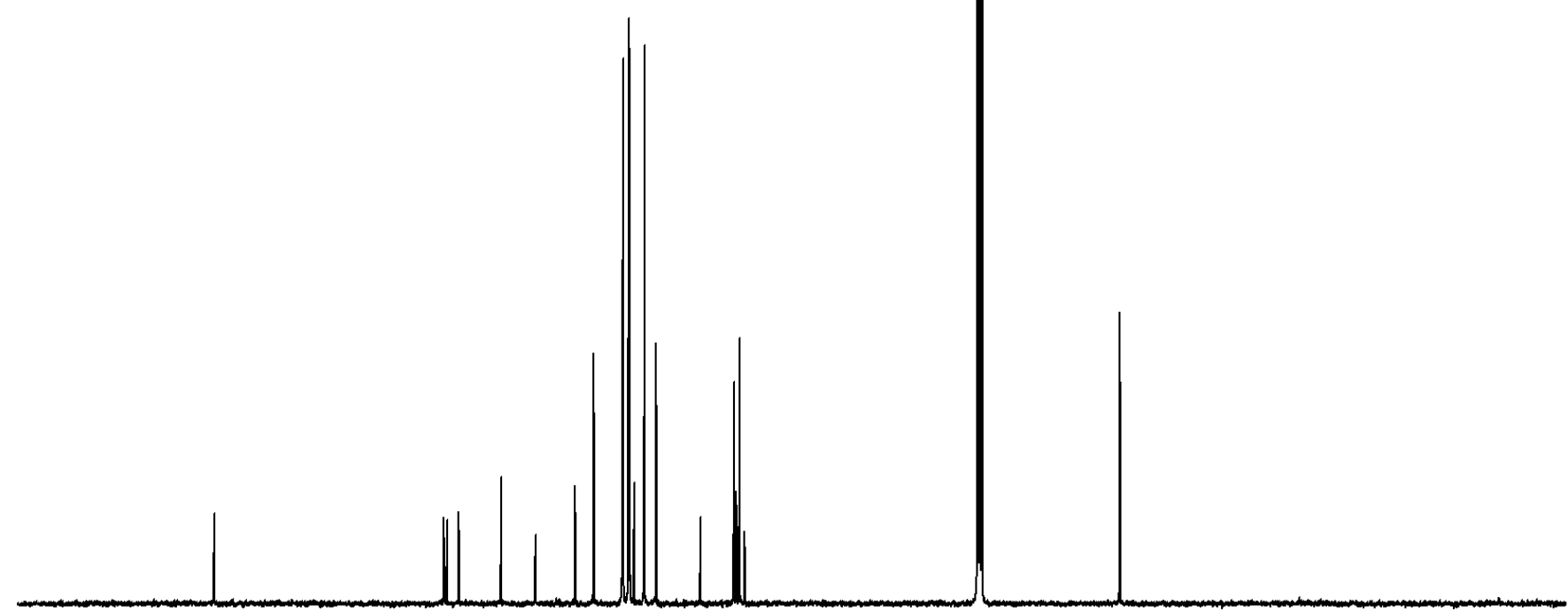

$=======$ CHANNEL f $1 \quad=======$
SFO1 $100.6228298 \mathrm{MHz}$

P1 10.00 use

PLW1 $49.50000000 \mathrm{~W}$

$=======$ CHANNEL $\mathrm{f} 2 \quad=======$
$\mathrm{SFO} 2 \quad 400.1316005 \mathrm{MHz}$

CPDPRG [ 2

PCPD2

PLW13 waltz16
90.00 use

$12.50000000 \mathrm{~W}$

$0.34722000 \mathrm{~W}$

2 - Processing parameters

$\begin{array}{ll}\text { SI } & 32768 \\ \text { SF } & 100.6127731 \mathrm{MHz}\end{array}$

WDW

LB

PB

$\mathrm{EM}$
0
$2.00 \mathrm{~Hz}$

1.00 
$\underline{{ }^{1} \mathrm{H} \text { NMR Spectrum of } 4 \mathrm{ia}\left(\mathrm{CDCl}_{3}, 400 \mathrm{MHz}\right)}$

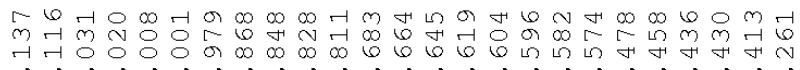

$\longrightarrow$

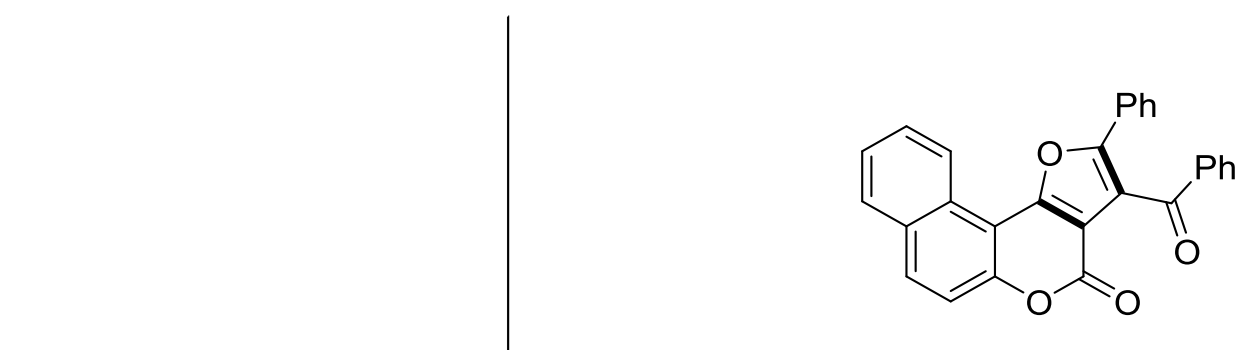

4ia

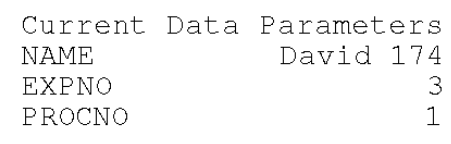

F2 - Acquisition Parameters

Date_ 20200717

Time

11.50

INSTRUM

PROBHD

PULPROG

TD

SOLVENT

NS

DS

SWH

FIDRES

AQ

RG

DW

$\mathrm{DE}$

D1

TDO

mm $\mathrm{BBO}$ spect

$\mathrm{BB}-1 \mathrm{H}$

zg30

$\mathrm{CDCl} 3$

0
$7246.377 \mathrm{~Hz}$

$0.221142 \mathrm{~Hz}$ $2.2609921 \mathrm{sec}$

$$
362
$$

69.000 usec

6.50 usec

$297.3 \mathrm{~K}$

$2.00000000 \mathrm{sec}$

$\begin{array}{lr}=======\text { CHANNEL } \mathrm{f} 1======= \\ \text { NUC1 } & 1 \mathrm{H} \\ \text { P1 } & 15.00 \mathrm{usec} \\ \text { PL1 } & 11.10 \mathrm{~dB} \\ \text { SF01 } & 11.008 \mathrm{uHz}\end{array}$

SFO1 $400.1324008 \mathrm{MHz}$

F2 - Processing parameters

SI 16384

SF $\quad 400.1300115 \mathrm{MHz}$

WDW

SSB

$\mathrm{LB}$

GB

400.1300115

EM
0

$\mathrm{O} \mathrm{Hz}$

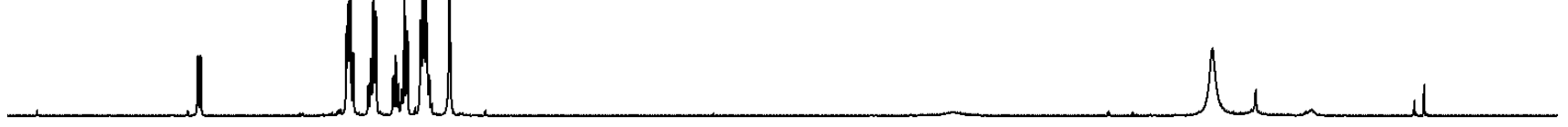

1.00

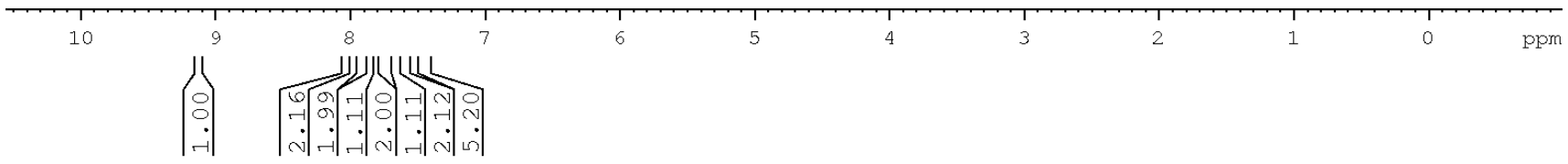


${ }^{13} \mathrm{C}$ NMR Spectrum of $4 \mathbf{i a}\left(\mathrm{CDCl}_{3}, 100 \mathrm{MHz}\right)$

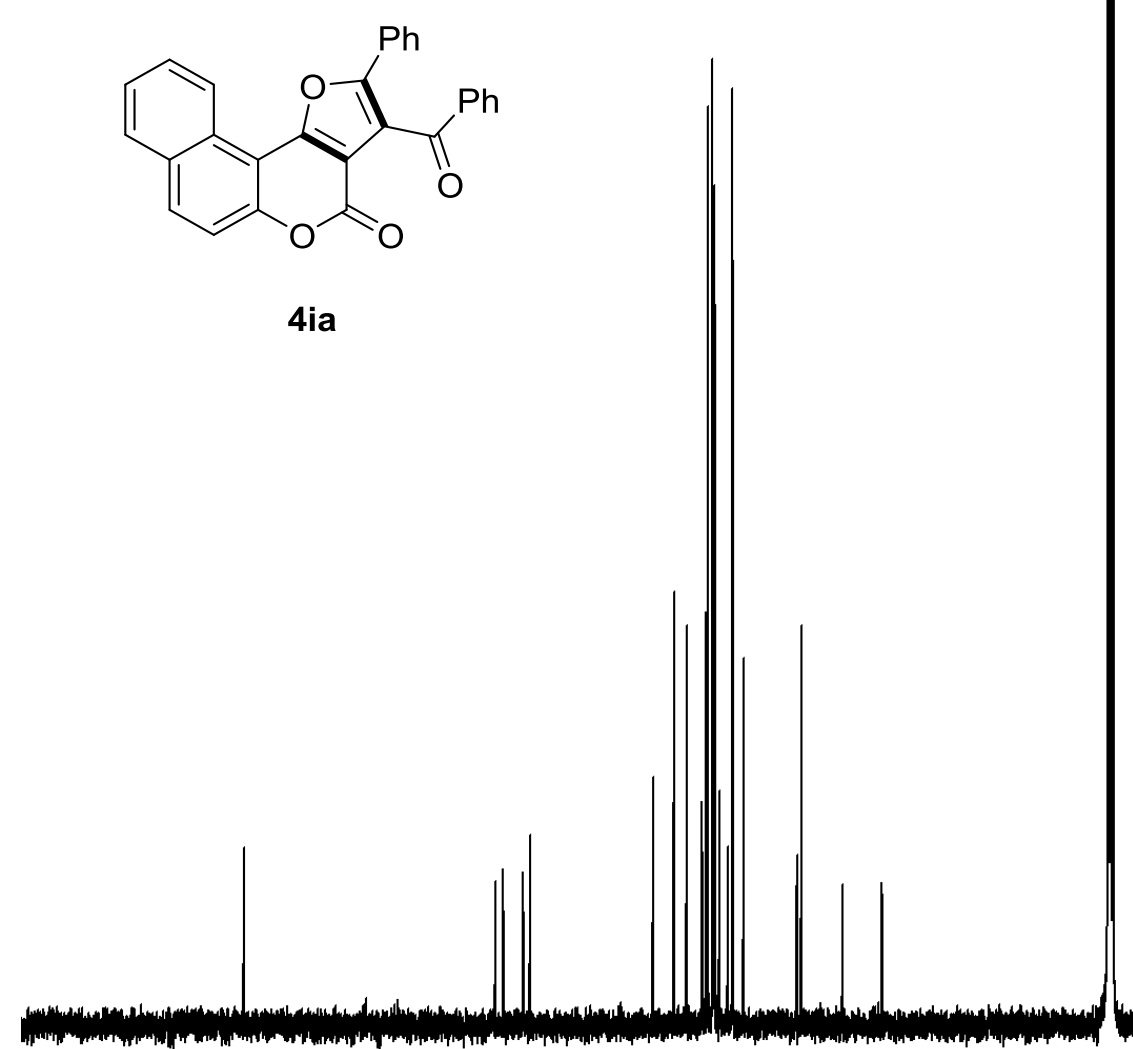

Current Data Parameters
NAME
David 174

EXPNO

PROCNO

2
1

F2 - Acquisition Parameters

Date__ 20200716

INSTRUM spect

PROBHD $5 \mathrm{~mm}$ BBO $\mathrm{BB}-1 \mathrm{H}$

PULPROG
zgpg30

S

DS

SWH

FIDRE
$\mathrm{AO}$

RG

DW

D1

D 11

$====$
NUC1
P1

$\mathrm{P} 1$

SFO

$\mathrm{CDCl} 3$
14586

0
$24038.461 \mathrm{~Hz}$
$0.733596 \mathrm{~Hz}$

$0.733596 \mathrm{~Hz}$ $0.6815744 \mathrm{sec}$

20.800 usec
6.50 usec

$2.00000000 \mathrm{sec}$

$1 \mathrm{sec}$

CHANNEL $\mathrm{f} 1$ $13 \mathrm{C}$

10.00 use
$3.80 \mathrm{~dB}$ $3.80 \mathrm{~dB}$
$100.6233325 \mathrm{MHz}$

$=======$ CHANNEL $\mathrm{f} 2$

CPDPRG [2 waltz16

$\begin{array}{lr}\text { PCPD2 } & 1 \mathrm{H} \\ \text { PIsec }\end{array}$

$10.20 \mathrm{~dB}$

$26.00 \mathrm{~dB}$

PL13

$400.1316005 \mathrm{MHz}$

F2 - Processing parameters

F2 - Processing parameters

$\begin{array}{lr}\text { SF } & 100.6127692 \\ \text { WDW } & \text { EM }\end{array}$

$\begin{array}{lc}\text { WDW } & \text { EM } \\ \text { SSB } & 0 \\ \text { LB } & 1.00 \mathrm{~Hz}\end{array}$

$\mathrm{LB}$
$\mathrm{GB}$
$\mathrm{PC}$

1.00
1.00 
${ }^{1} \mathrm{H}$ NMR Spectrum of $4 \mathbf{a b}\left(\mathrm{CDCl}_{3}, 400 \mathrm{MHz}\right)$

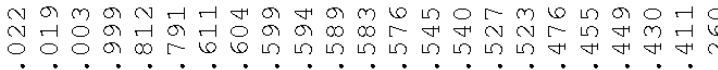

$\longrightarrow$

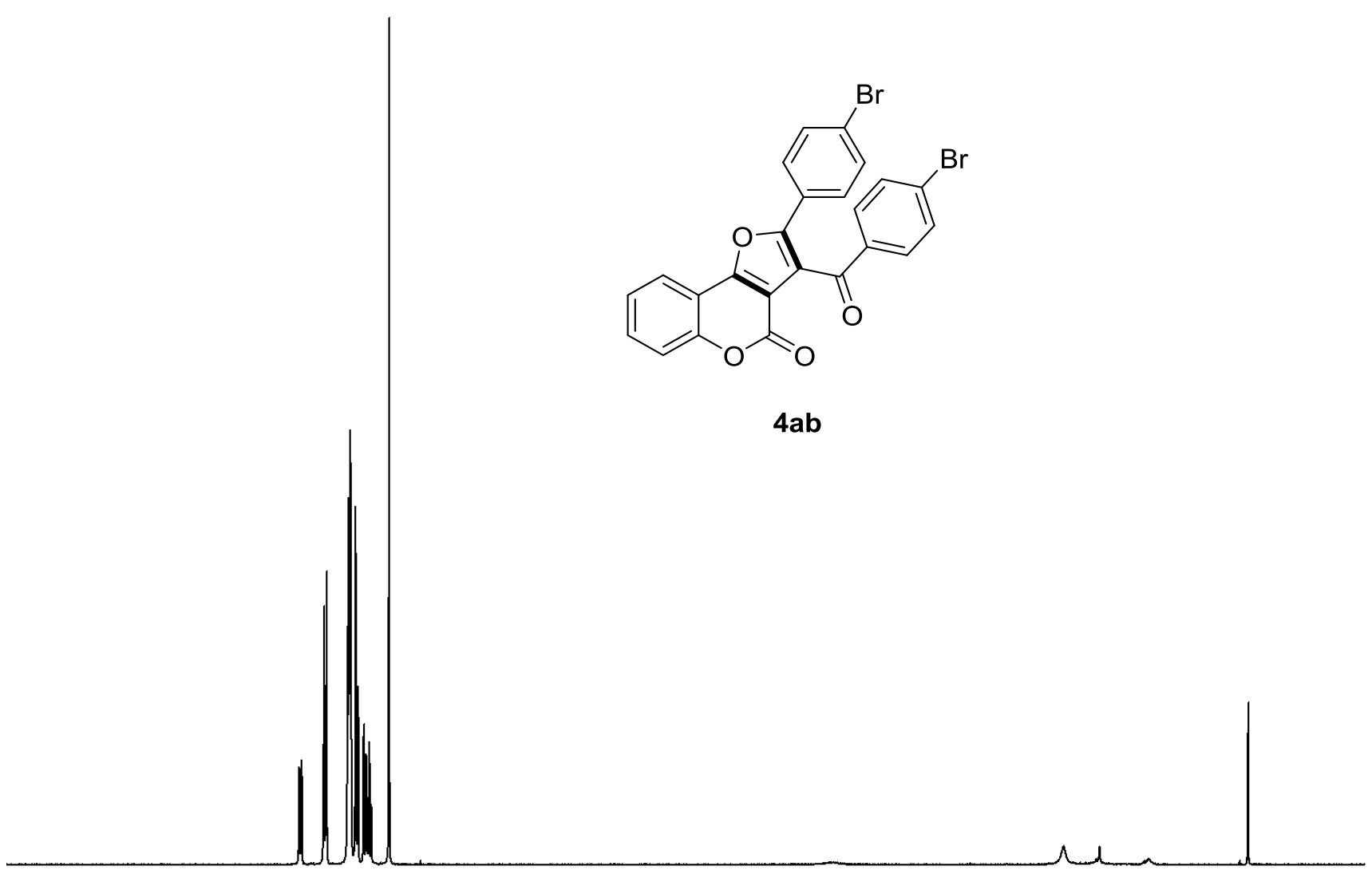

Current Data Parameters

NAME

EXPNO

SW 858

PROCNO

F2 - Acquisition Parameters

Date__ 20200716

Time

0.57

INSTRUM

ROBHD

$5 \mathrm{~mm}$ BBO $\mathrm{BB}-1 \mathrm{H}$

SOLVENT

NS

DS

SWH

FIDRES

AQ

RG

DW

$\mathrm{DE}$

D1

TDO $\mathrm{zg} 30$ $\mathrm{CDCl} 3$

0
7246.377
$0 z$

$0.221142 \mathrm{~Hz}$

2.2609921 sec

322.5

69.000 usec

6.50 usec

$298.0 \mathrm{~K}$

$2.00000000 \mathrm{sec}$

$\begin{array}{lr}=======\text { CHANNEL } \mathrm{fl}======= \\ \text { NUC1 } & 1 \mathrm{H} \\ \text { P1 } & 15.00 \text { usec } \\ \text { PL1 } & 11.10 \mathrm{~dB} \\ \text { SE01 } & \end{array}$

$400.1324008 \mathrm{MHz}$

F2 - Processing parameters

SI 16384

SF $\quad 400.1300110 \mathrm{MHz}$

WDW

SSB

LB

GB

400.1300110

EM

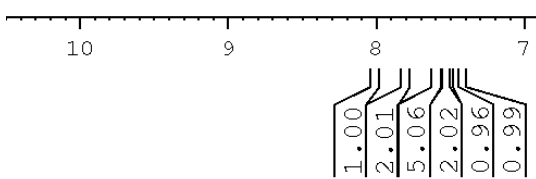


${ }^{13} \mathrm{C}$ NMR Spectrum of $4 \mathbf{a b}\left(\mathrm{CDCl}_{3}, 100 \mathrm{MHz}\right)$

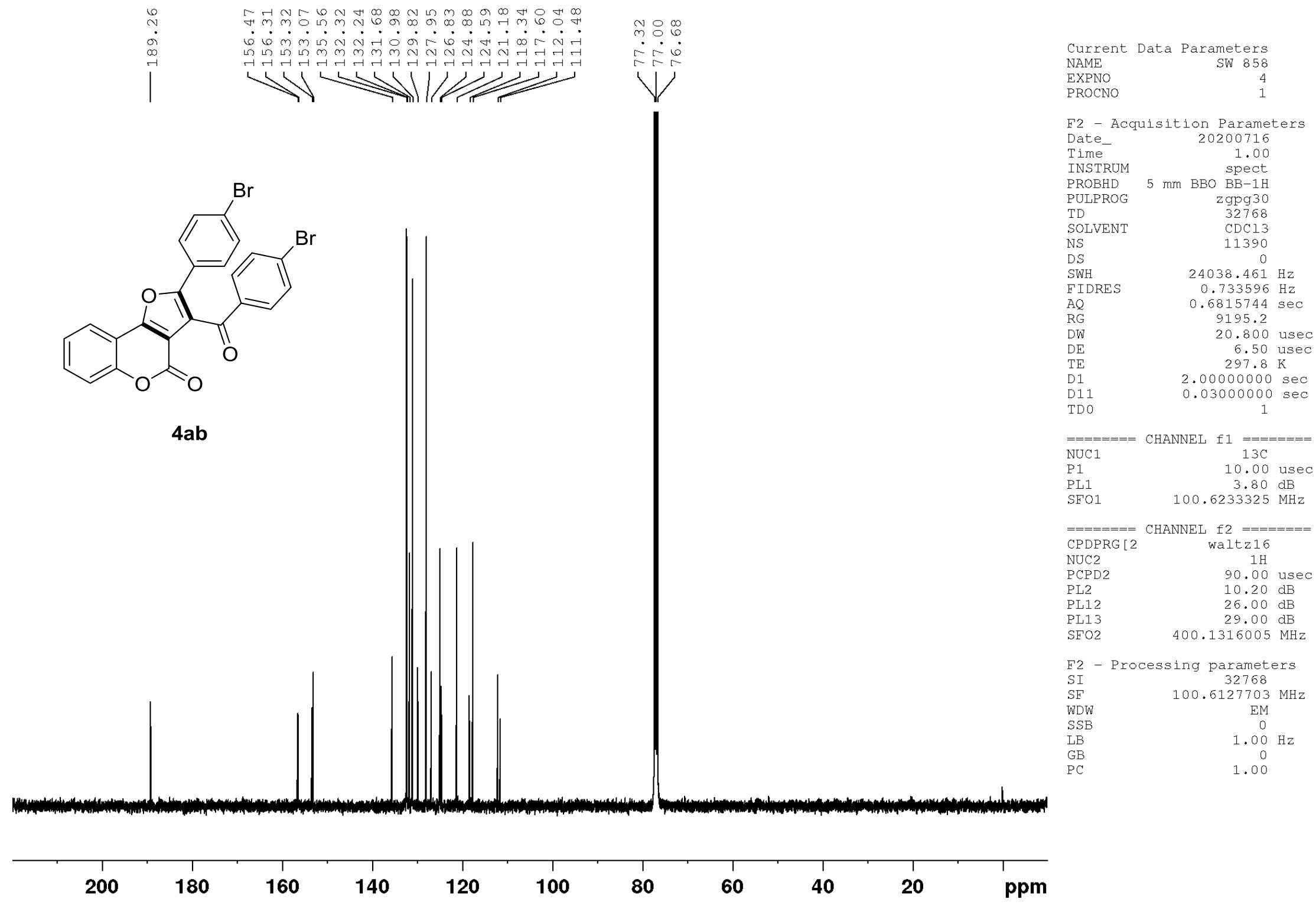


$\underline{{ }^{1} \mathrm{H} \text { NMR Spectrum of } \mathbf{4 a c}\left(\mathrm{CDCl}_{3}, 400 \mathrm{MHz}\right)}$

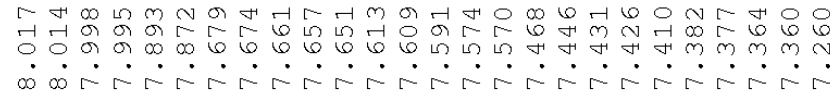

$\pi$

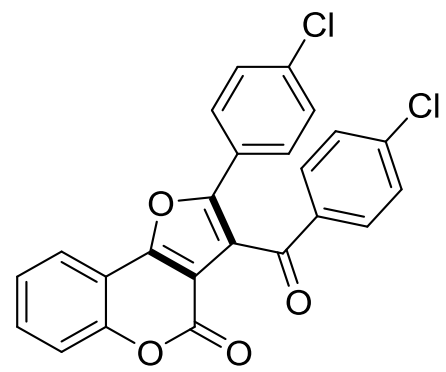

4ac

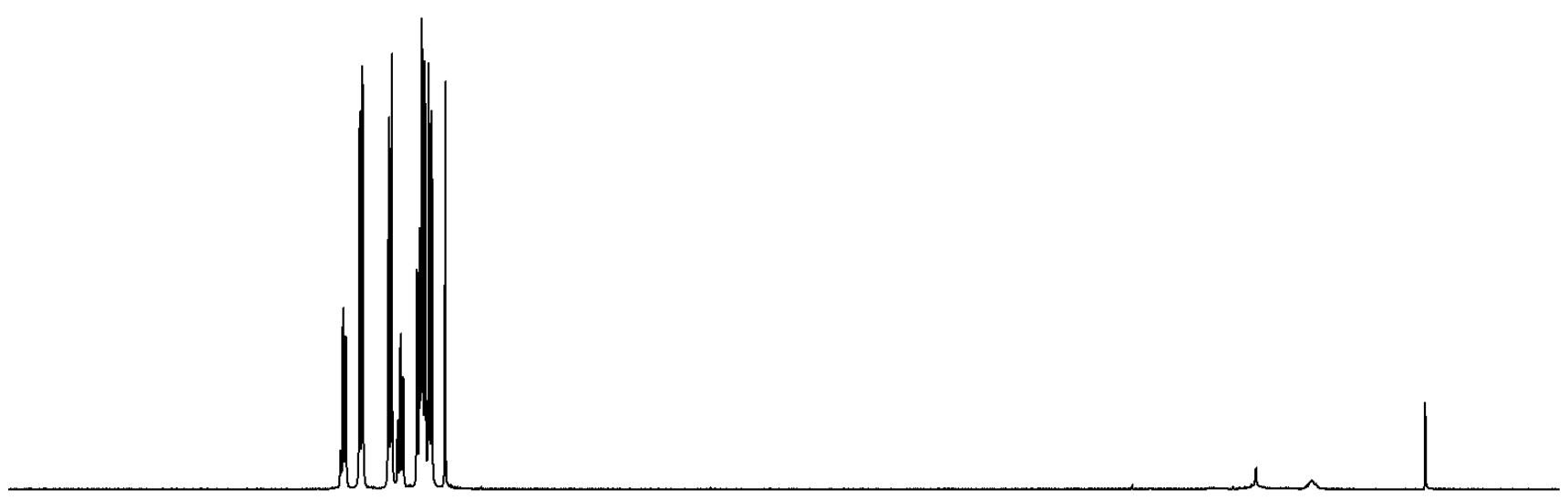

Current Data Parameters

Current David 171 (3f)

XPNO

PROCNO

F2 - Acquisition Parameters

Date__ 20200801

Time

0.56

INSTRUM

INSTRUM

PULPROG

spect

TD

SOLVENT

NS

DS

SWH

FIDRES

$A Q$
$R G$
DW

DW

$\mathrm{DE}$

DE

TD 1

SEO1

NUC

P1

$5 \mathrm{~mm}$ PABBO $\mathrm{BB} /$

$\mathrm{zg} 30$
32768

$\mathrm{CDCl} 3$

0
$7211.539 \mathrm{~Hz}$

$0.220079 \mathrm{~Hz}$

$2.2719147 \mathrm{sec}$

198.09

69.333 usec

10.06 usec

$2.00000000 \mathrm{sec}$

CHANNEL $\mathrm{f} 1$ 1

PLW1

00.1324008 mHz $1 \mathrm{H}$ 15.00 usec

$11.39999962 \mathrm{~W}$

F2 - Processing parameters

SI 16384

SF $\quad 400.1300096 \mathrm{MHz}$

WDW

SSB
$\mathrm{LB}$

$\mathrm{LB}$
$\mathrm{GB}$

$\mathrm{EM}$

$\mathrm{PC}$

1.00

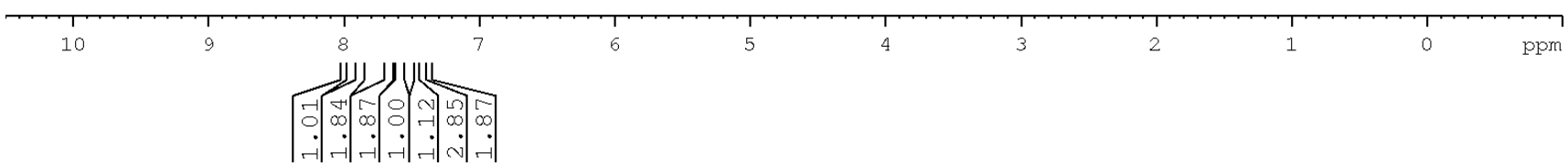


${ }^{13} \mathrm{C}$ NMR Spectrum of $4 \mathrm{ac}\left(\mathrm{CDCl}_{3}, 100 \mathrm{MHz}\right)$
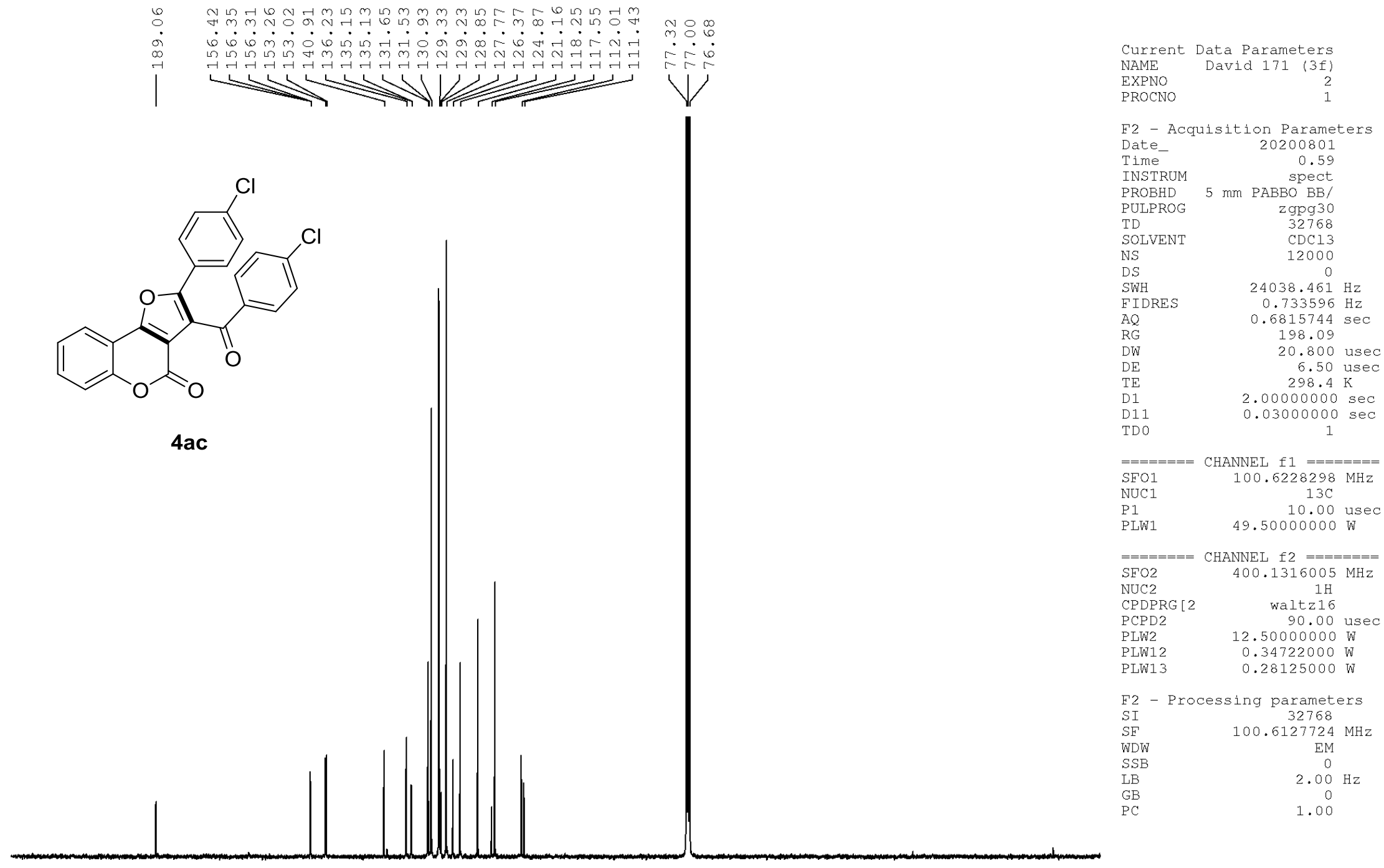

200

180

160

140

120

100

80

60

40

20

ppm 
${ }^{1} \mathrm{H}$ NMR Spectrum of $\mathbf{4 a d}\left(\mathrm{CDCl}_{3}, 400 \mathrm{MHz}\right)$

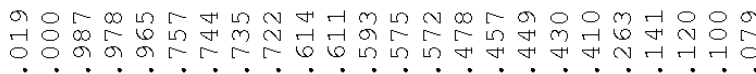

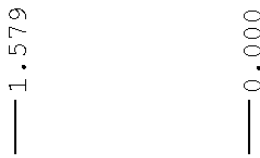

Current Data Parameters

NAME David 179-1 (3f)

TXPNO

EXPNO

3
1

F2 - Acquisition Parameters

Date__ 20200801

Time

18.24

INSTRUM

PROBHD

PULPROG

mm $\mathrm{PABBO} \mathrm{BB} /$

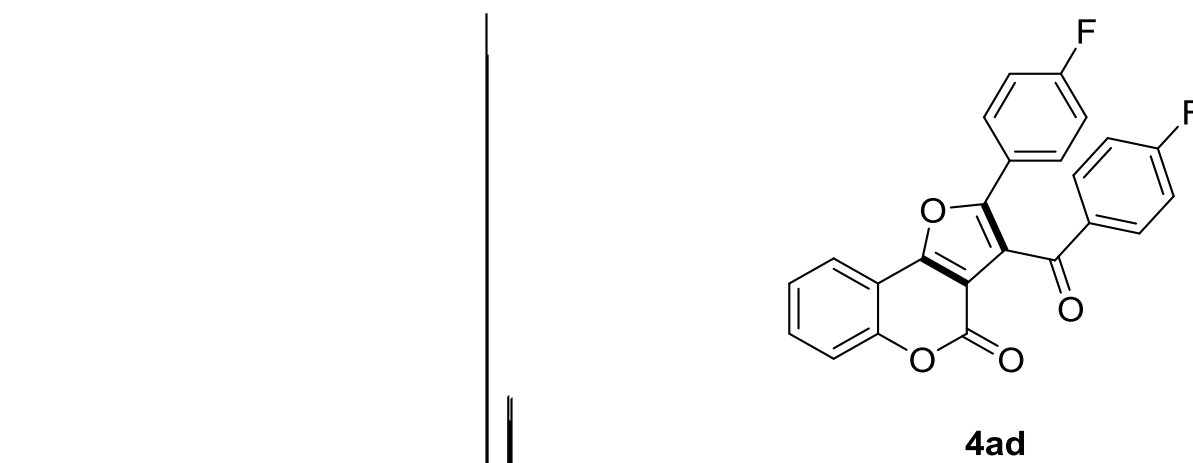

SOLVENT

NS

DS

SWH

FIDRES

$\mathrm{AQ}$
$\mathrm{RG}$

DW

DE

DE

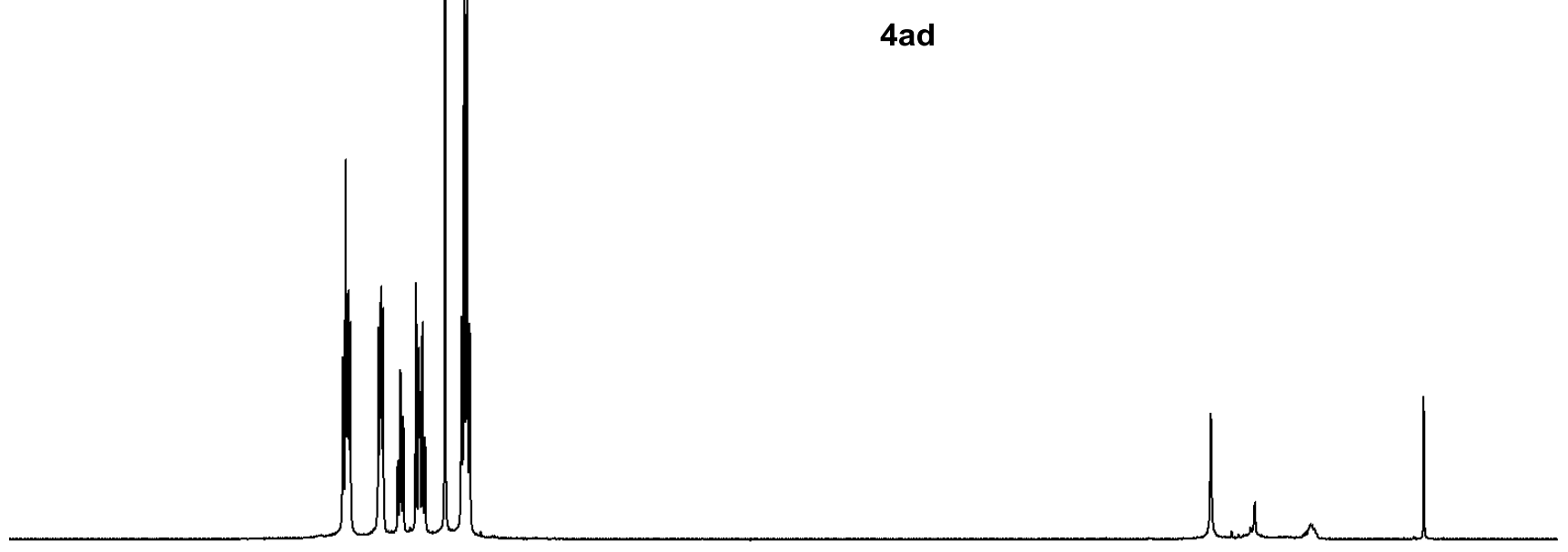

DO

$====$

$\mathrm{SEO}$

NUC

$\mathrm{zg} 30$

$\mathrm{zg} 30$
32768

0
$7211.539 \mathrm{~Hz}$

$0.220079 \mathrm{~Hz}$

$2.2719147 \mathrm{sec}$

198.09

69.333 usec

10.06 usec

$2.00000000 \mathrm{sec}$

LW1

HANNEL $\mathrm{f} 1=======$ $400.1324008 \mathrm{MHz}$

$1 \mathrm{H}$

5.00 usec

F2 - Processing parameters

SI 16384

SF $\quad 400.1300087 \mathrm{MHz}$

WDW

SSB
LB

$\mathrm{PC}$

EM
0
0
0
0
1.00

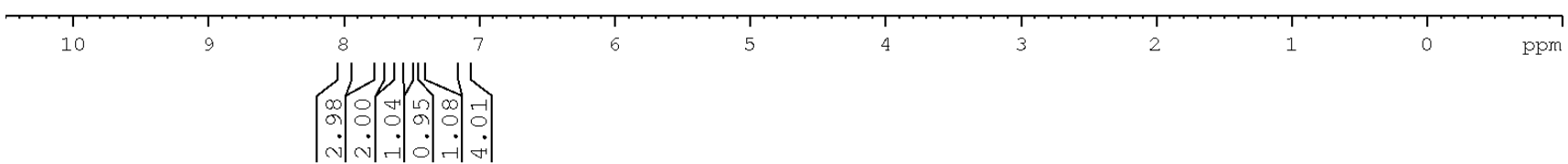


${ }^{13} \mathrm{C}$ NMR Spectrum of 4 ad $\left(\mathrm{CDCl}_{3}, 100 \mathrm{MHz}\right)$

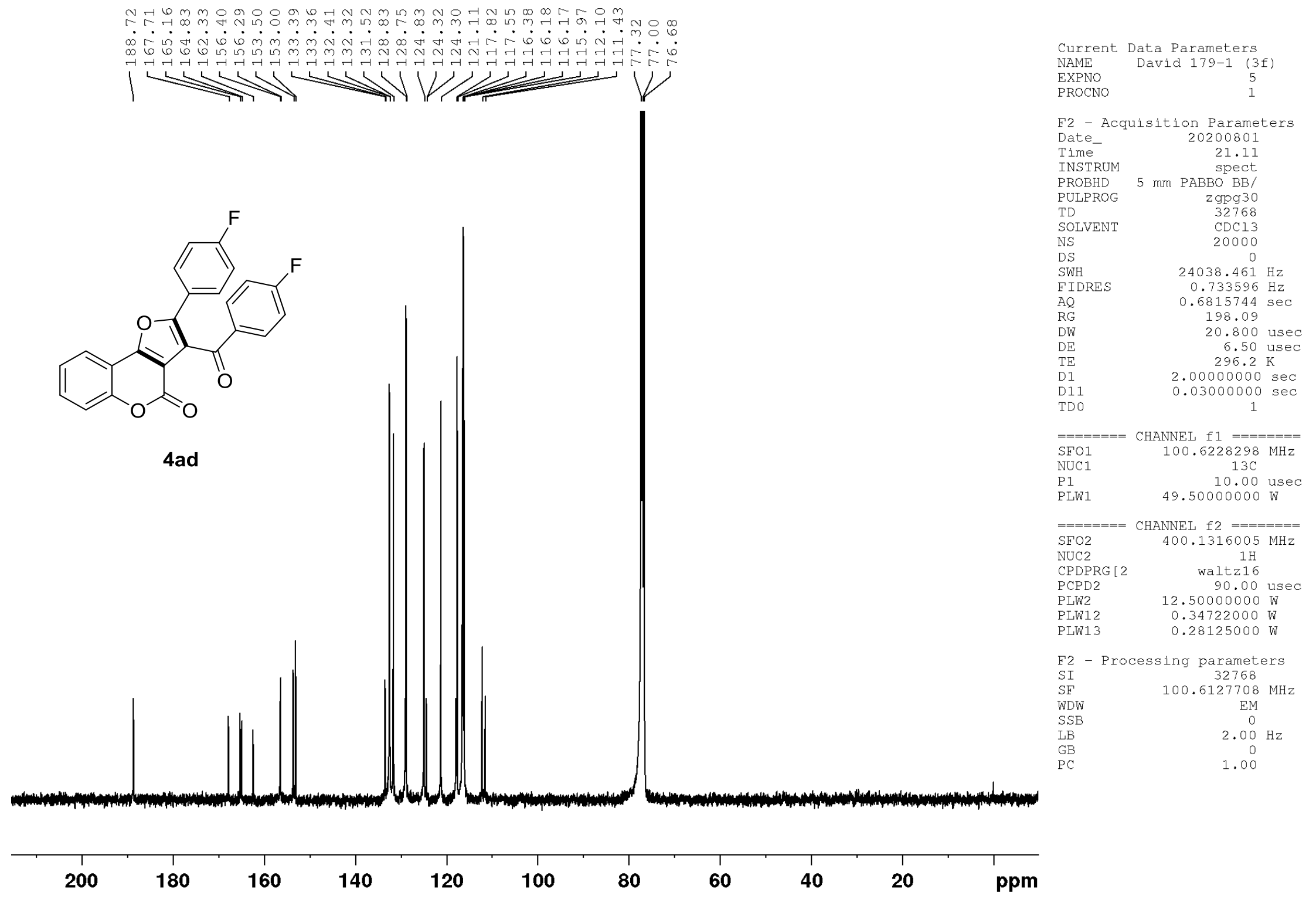




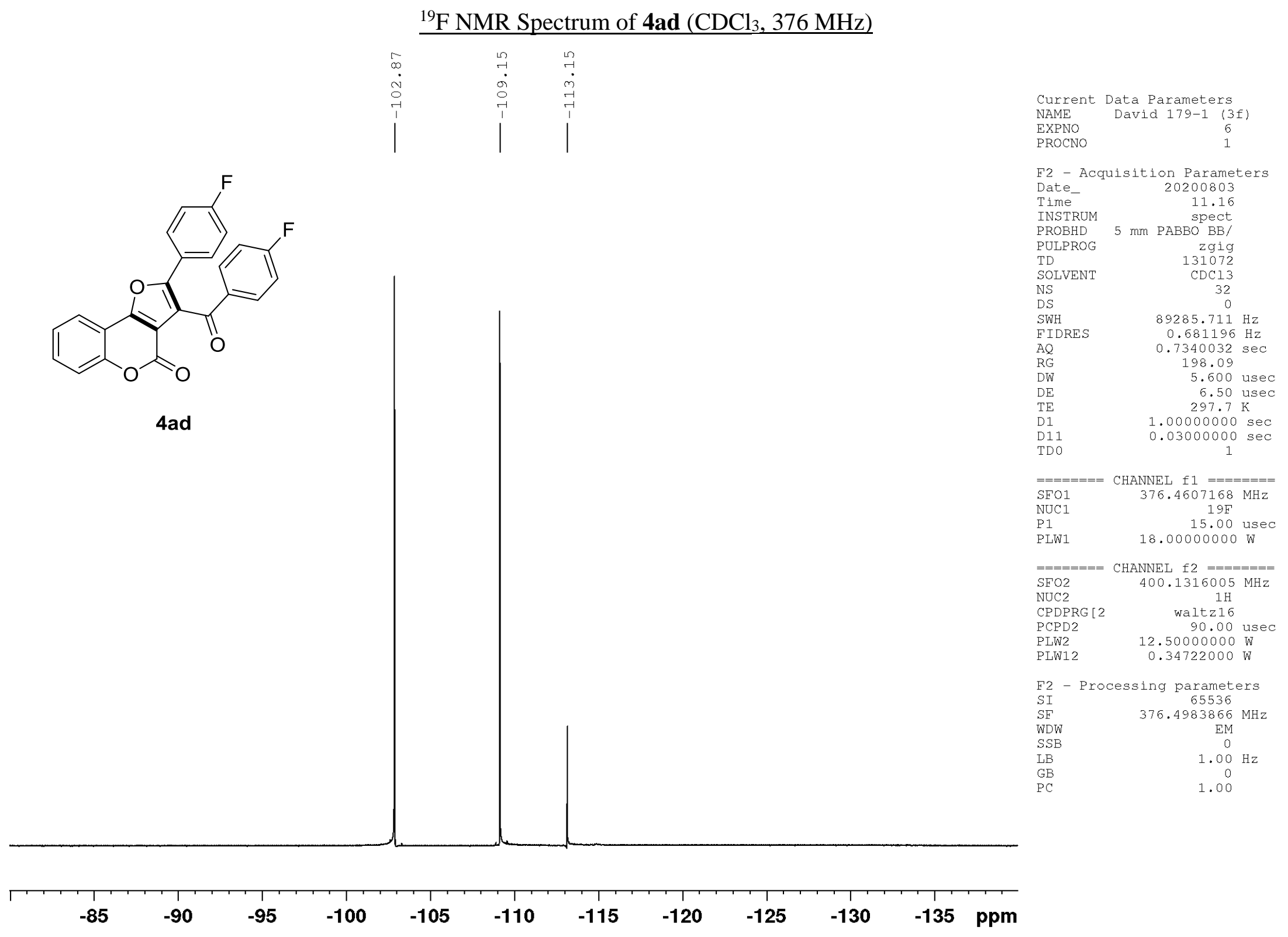


$\underline{{ }^{1} \mathrm{H} \text { NMR Spectrum of } 4 \mathrm{ae}\left(\mathrm{CDCl}_{3}, 400 \mathrm{MHz}\right)}$

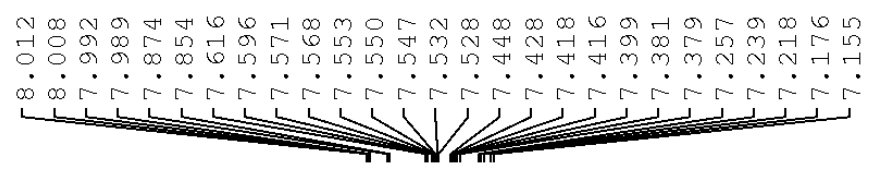

$\begin{array}{ll}9 & \infty \\ \infty & m \\ m & m \\ ن & \dot{N}\end{array}$

Current Data Parameters

NAME

EXPNO
PROCNO

Carol 009 (3f)

2 -

Acquisition Parameters

Time

20200728

INSTRUM

PULPROG

TD

SOLVENT

NS

DS

SWH

FIDRES

AQ

DW

$\mathrm{DE}$

TE

4 ae

TDO

$======$

$\mathrm{SEO}$

NUC1

P1

spect

$5 \mathrm{~mm}$ PABBO BB/

$\mathrm{zg} 30$
32768

$\mathrm{CDCl} 3$

0
$7211.539 \mathrm{~Hz}$
$0.220079 \mathrm{~Hz}$

$0.220079 \mathrm{~Hz}$ 2.2719147
89.08

69.333 usec

10.06 usec

$296.6 \mathrm{~K}$

$2.00000000 \mathrm{sec}$

$$
1
$$

CHANNEL $\mathrm{f} 1=======$ $400.1324008 \mathrm{MHz}$

15.00
us

11.39999962 W

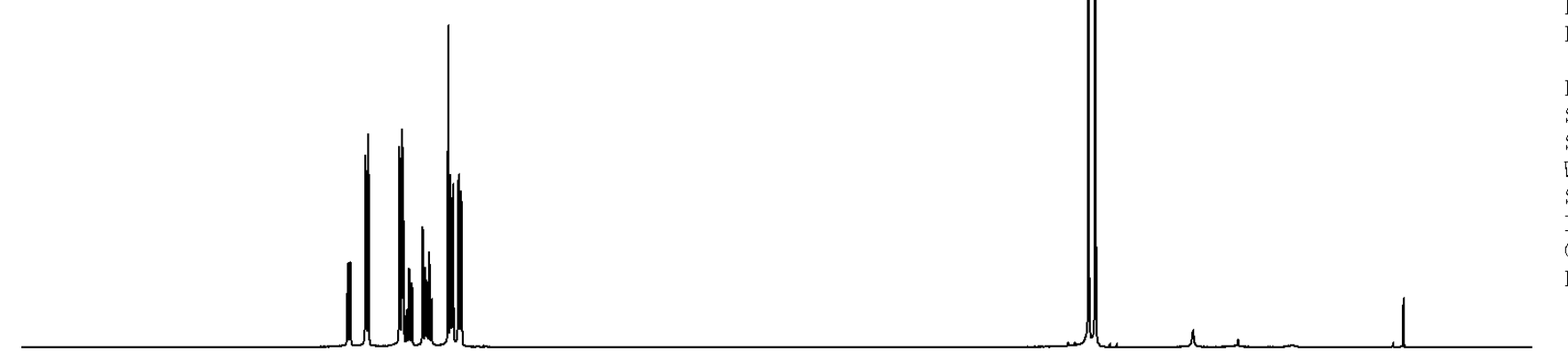

F2 - Processing parameters

SI 16384

SF $\quad 400.1300111 \mathrm{MHz}$

WDW

SSB
LB

LB
GB

$\mathrm{PC}$

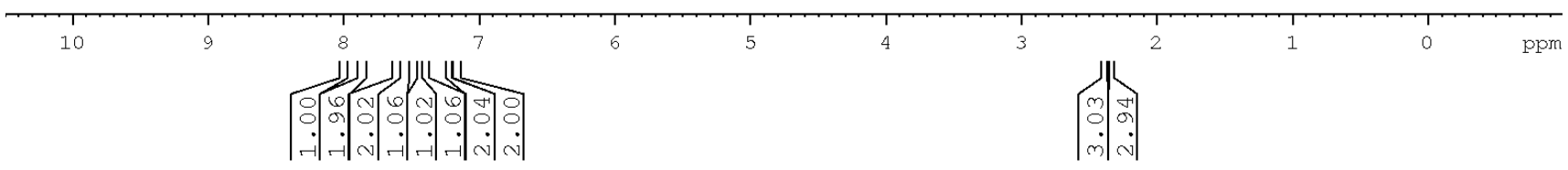


${ }^{13} \mathrm{C}$ NMR Spectrum of $4 a e\left(\mathrm{CDCl}_{3}, 100 \mathrm{MHz}\right)$

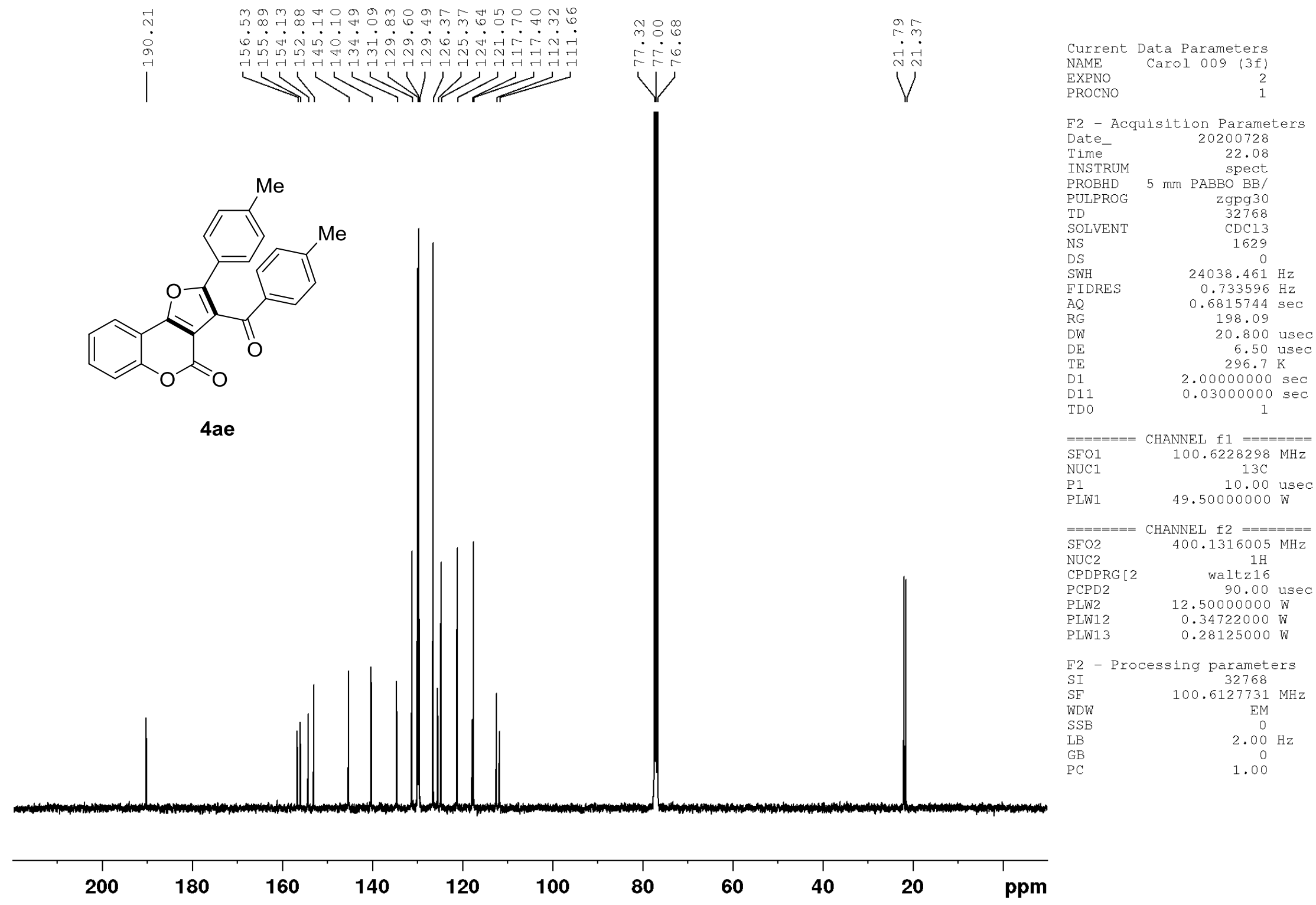


$\underline{{ }^{1} \mathrm{H} \text { NMR Spectrum of } 4 \mathbf{a f}\left(\mathrm{CDCl}_{3}, 400 \mathrm{MHz}\right)}$

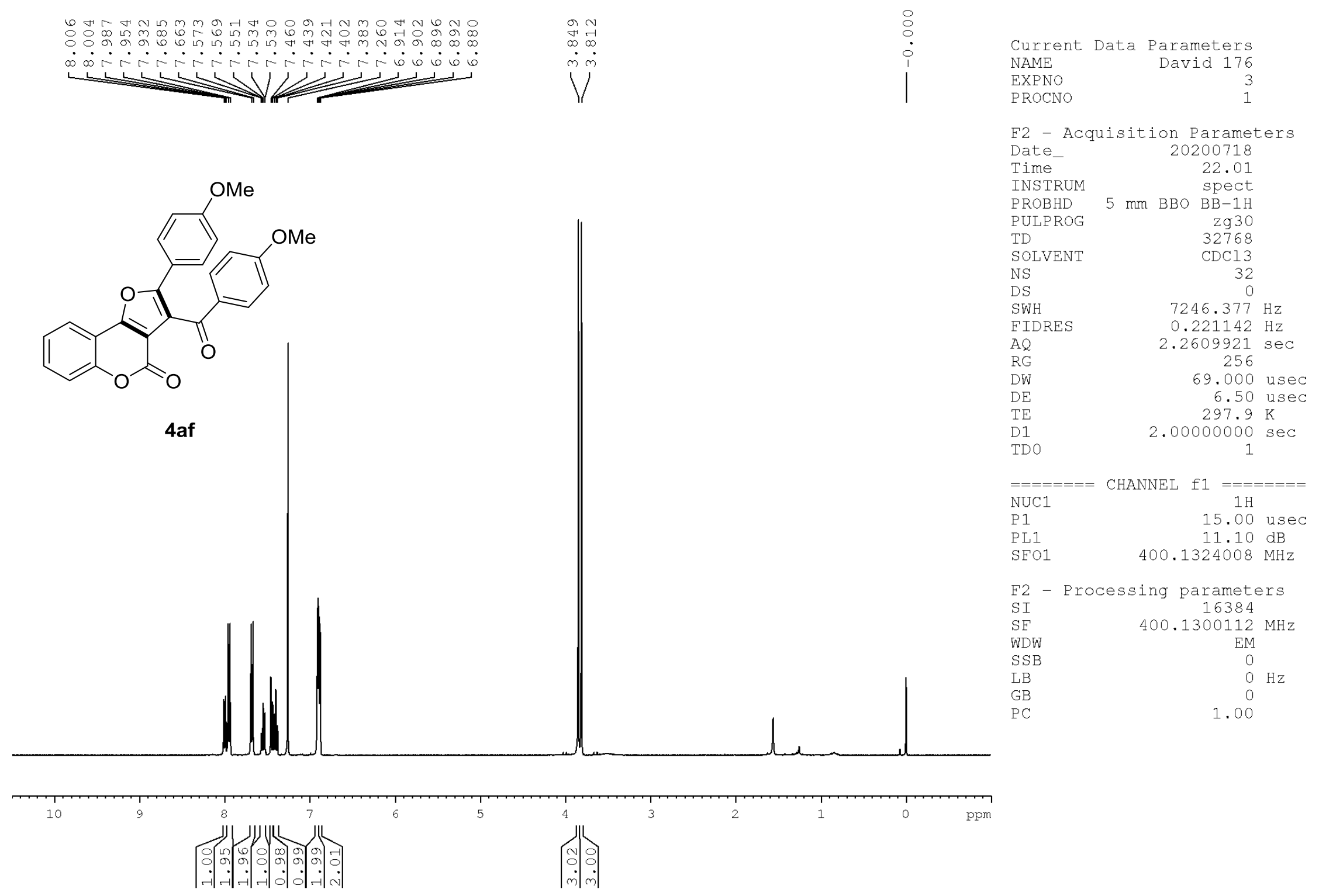


${ }^{13} \mathrm{C}$ NMR Spectrum of $4 \mathrm{af}\left(\mathrm{CDCl}_{3}, 100 \mathrm{MHz}\right)$
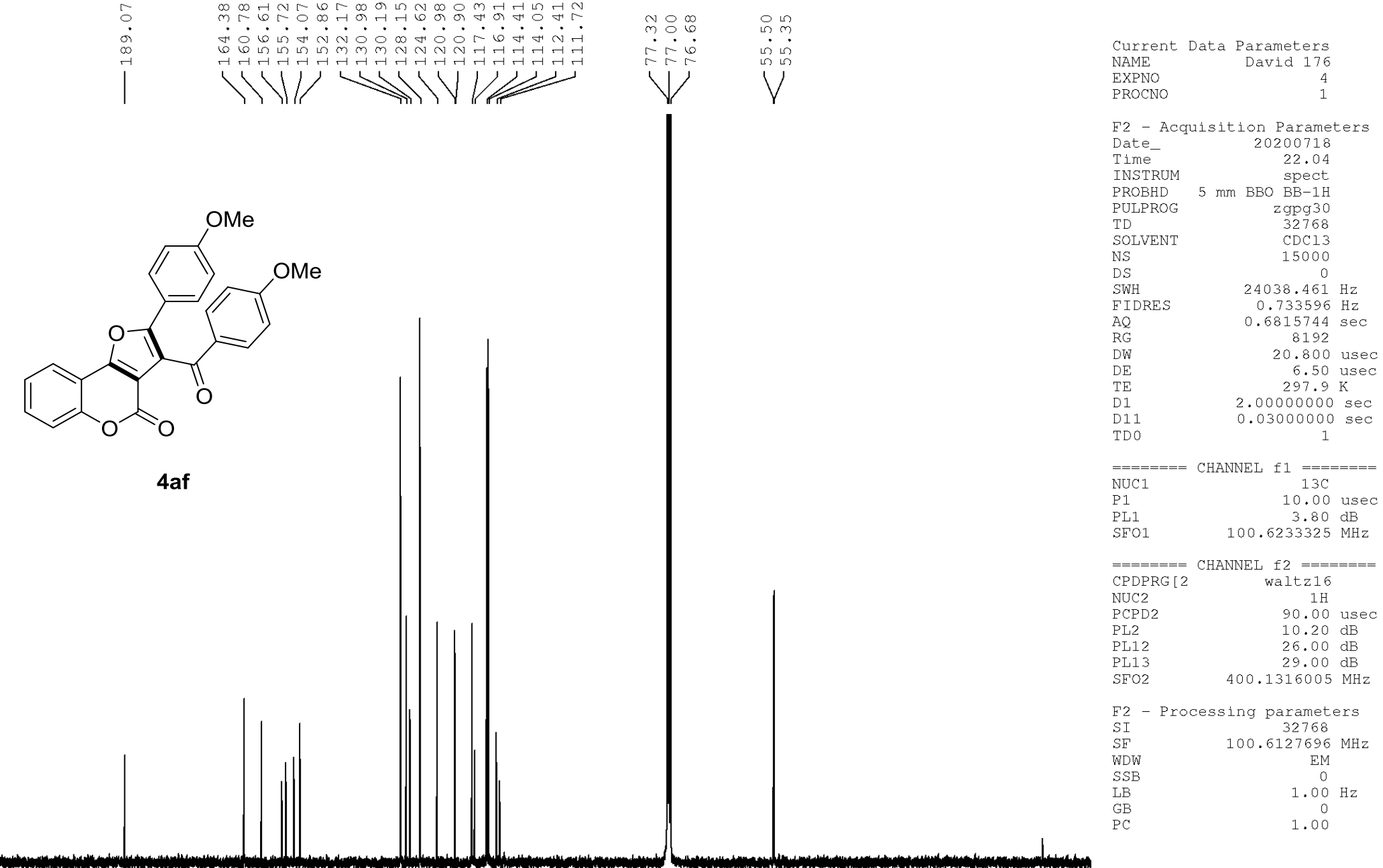
$\underline{{ }^{1} \mathrm{H} \text { NMR Spectrum of } \mathbf{4 a g}\left(\mathrm{CDCl}_{3}, 400 \mathrm{MHz}\right)}$

$\begin{array}{lrr}\text { Current } & \text { Data Parameters } \\ \text { NAME } & \text { David } 182(3 \mathrm{f}) \\ \text { EXPNO } & & 3 \\ \text { PROCNO } & & 1\end{array}$

F2 - Acquisition Parameters

Date_ 20200730

Time $\quad 23.22$

INSTRUM spect

PROBHD $5 \mathrm{~mm}$ PABBO BB/

PULPROG $\quad$ zg30

TD 32768

SOLVENT CDCl3

NS

DS

$\begin{array}{ll}\text { SWH } & 7211.539 \mathrm{~Hz} \\ \text { FIDRES } & 0.220079 \mathrm{~Hz}\end{array}$

$2.2719147 \mathrm{~Hz}$

$\begin{array}{lr}2.27 & 99.72\end{array}$

DW $\quad 69.333$ usec

DE $\quad 10.06$ usec

TE $\quad 297.8 \mathrm{~K}$

D1 $2.00000000 \mathrm{sec}$

4ag

$========$ CHANNEL $\mathrm{f} 1========$ SFO1 $\quad 400.1324008 \mathrm{MHz}$

$$
\begin{array}{lr}
\text { SFO1 } & 400.1324008 \\
\text { NUC1 } & 1 \mathrm{H}
\end{array}
$$

P 1 1

F2 - Processing parameters

SI 16384

SF $\quad 400.1300095 \mathrm{MHz}$

WDW

SSB

LB

GB

EM

U

1.00

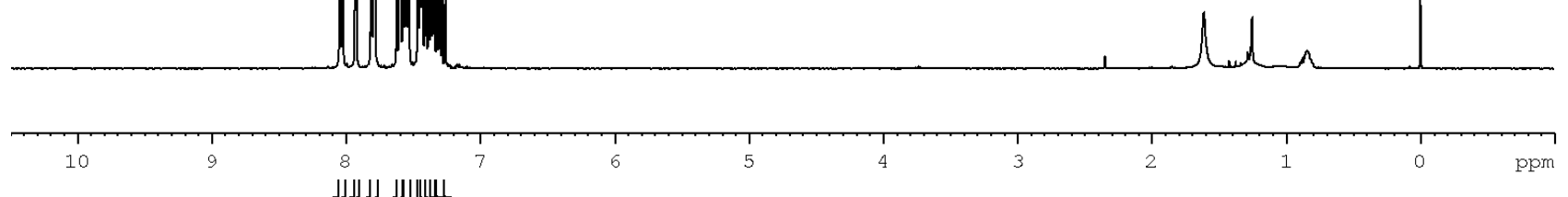

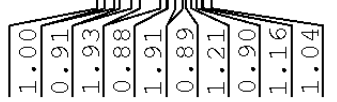


${ }^{13} \mathrm{C}$ NMR Spectrum of $4 \mathrm{ag}\left(\mathrm{CDCl}_{3}, 100 \mathrm{MHz}\right)$

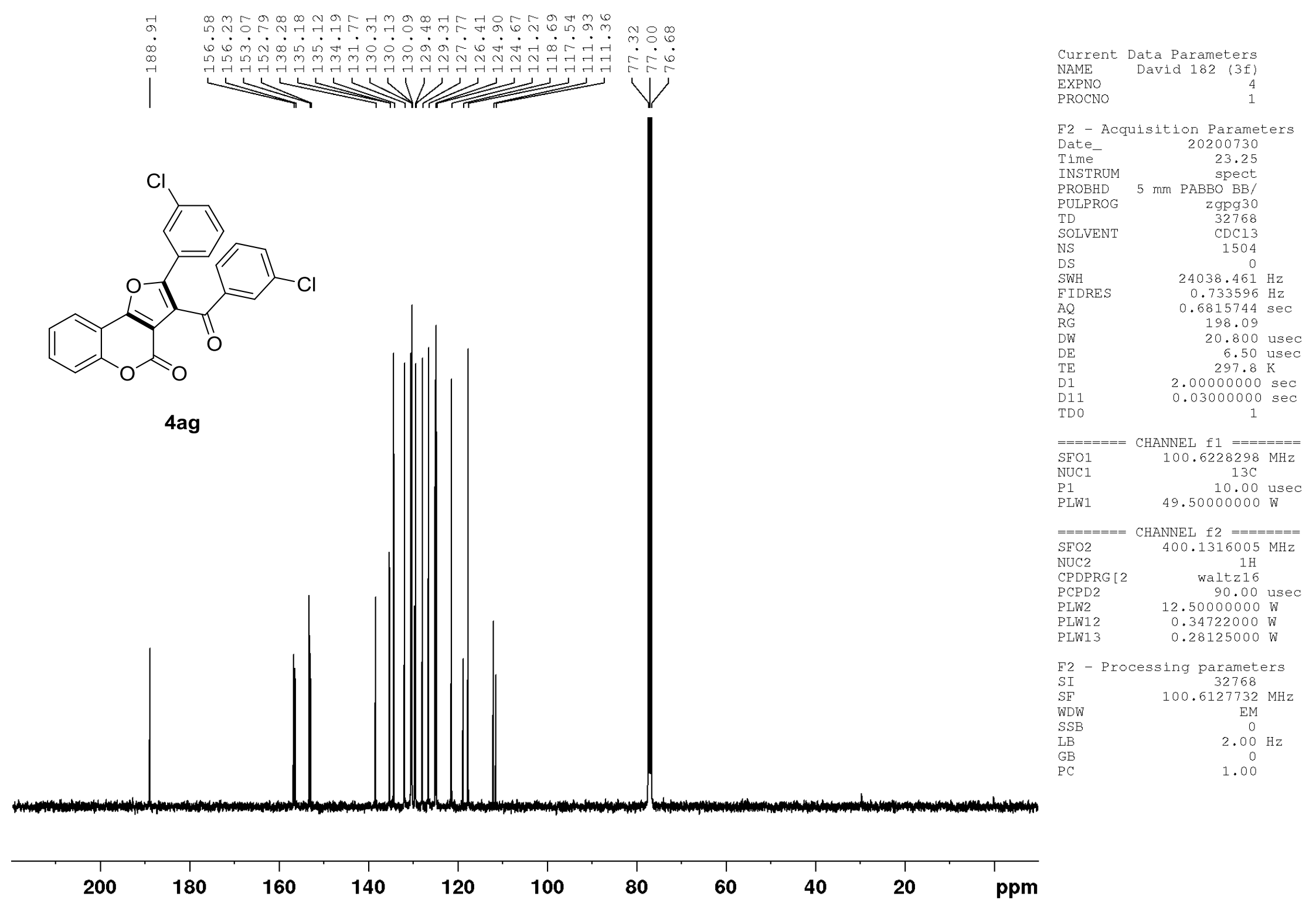


${ }^{1} \mathrm{H}$ NMR Spectrum of $4 \mathbf{a h}\left(\mathrm{CDCl}_{3}, 400 \mathrm{MHz}\right)$
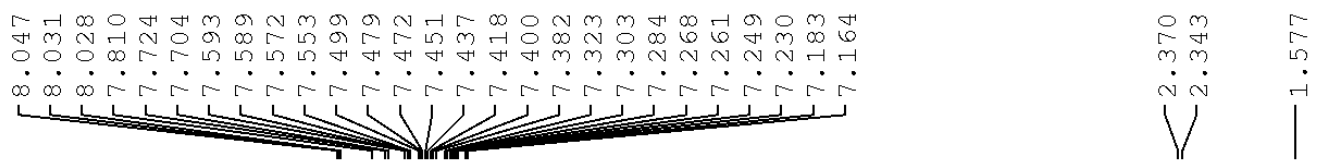

$\begin{array}{lr}\text { Current Data Parameters } \\ \text { NAME } & \text { Carol } 003 \\ \text { EXPNO } & 1 \\ \text { PROCNO } & 1\end{array}$

1

F2 - Acquisition Parameters

Date_ 20200714

Time

11.47

INSTRUM

PROBHD

PULPROG

TD
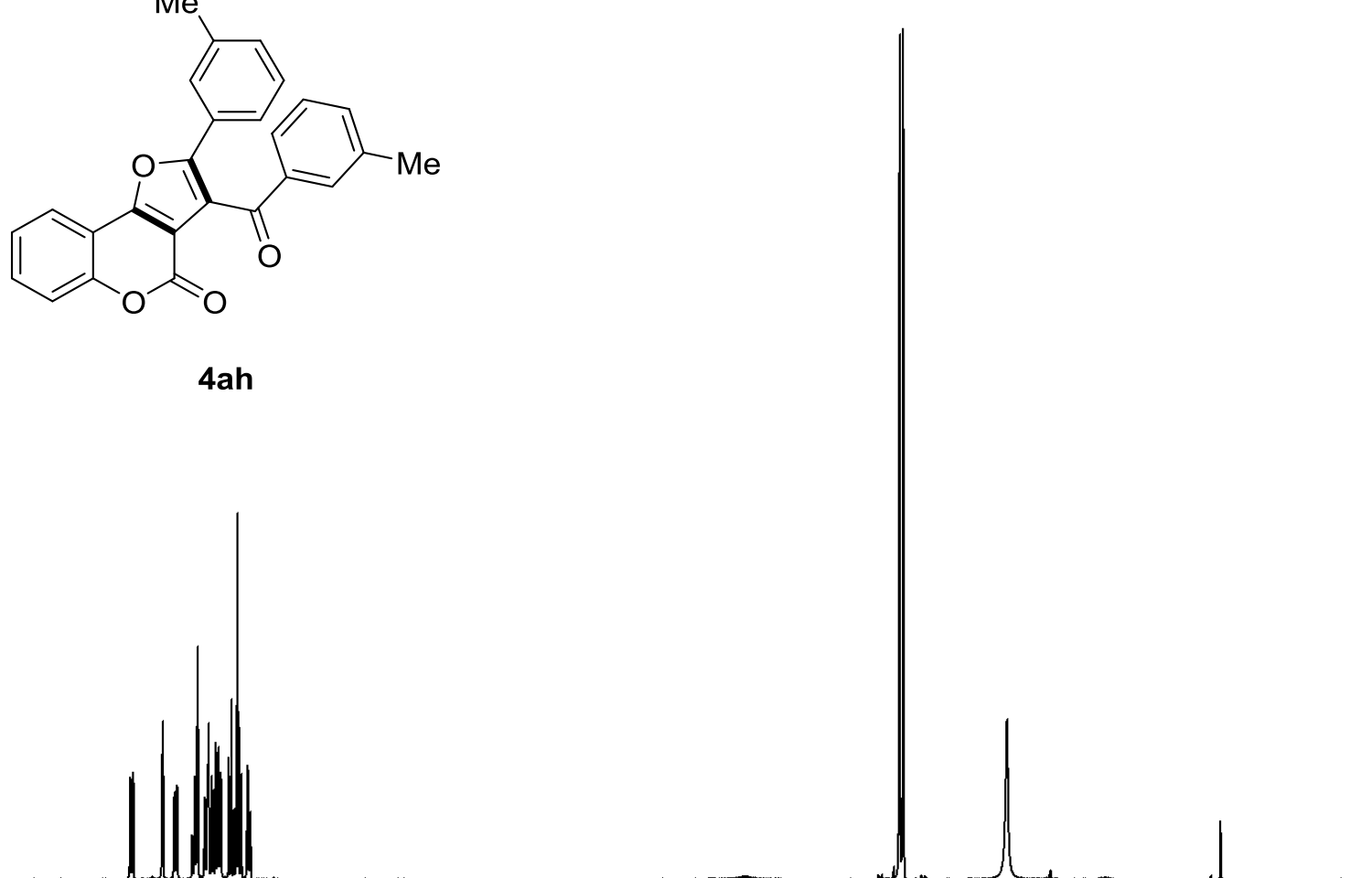

SOLVENT

NS

DS

SWH

FIDRES

AQ
RG
DW

DW

$\mathrm{DE}$

D1

4ah

TDO

$\begin{array}{lr}=======\text { CHANNEL } \mathrm{fl}======= \\ \text { NUC1 } & 1 \mathrm{H} \\ \text { P1 } & 15.00 \text { usec } \\ \text { PL1 } & 11.10 \mathrm{~dB} \\ \text { SE01 } & \end{array}$

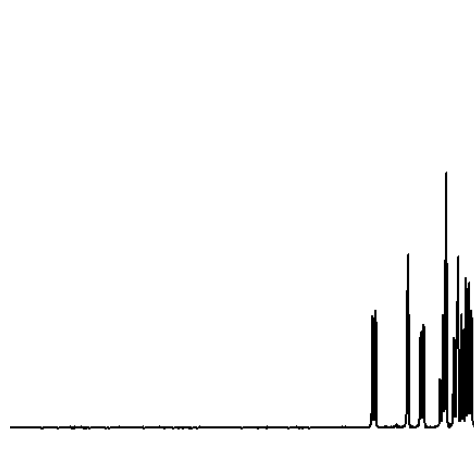

$\mathrm{SEO}$

$400.1324008 \mathrm{MHz}$

F2 - Processing parameters

SI 16384

SF $\quad 400.1300106 \mathrm{MHz}$

WDW

SSB
LB

LB

$\mathrm{PC}$

$\mathrm{EM}$

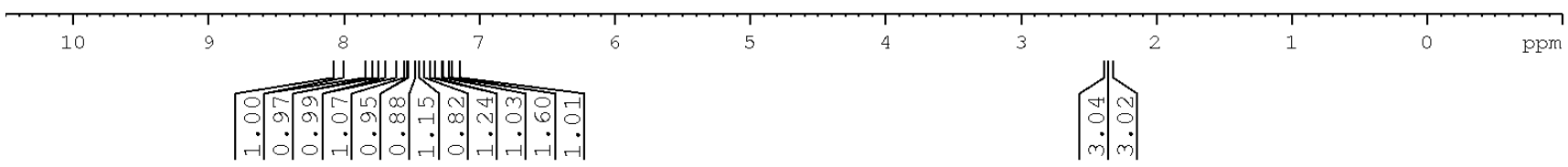




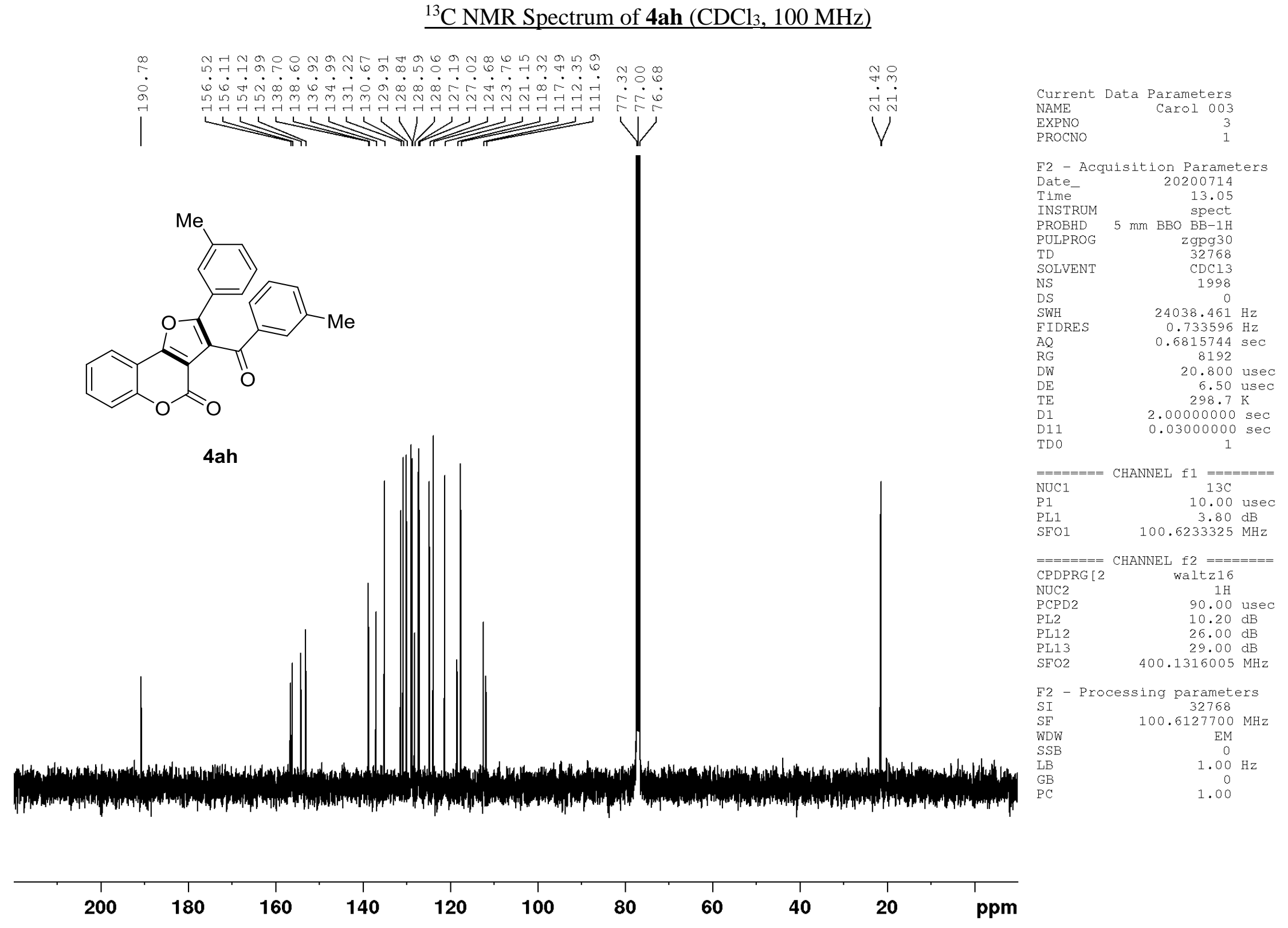


$\underline{{ }^{1} \mathrm{H} \text { NMR Spectrum of } 4 \mathbf{a i}\left(\mathrm{CDCl}_{3}, 400 \mathrm{MHz}\right)}$
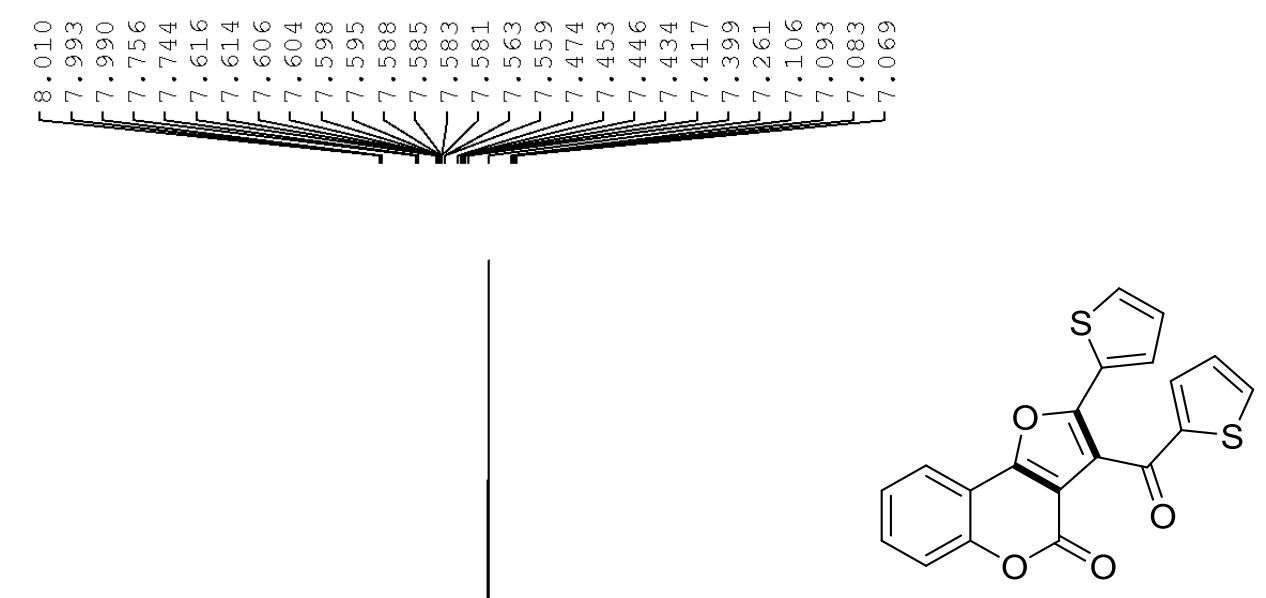

F2 - Acquisition Parameters

Date_ 20200715

Time 21.52

INSTRUM spect

PROBHD $5 \mathrm{~mm}$ BBO BB-1H

PULPROG $\quad$ zg30

TD 32768

$\mathrm{CDCl} 3$

NS

SWH $\quad 7246.377 \mathrm{~Hz}$

FIDRES $\quad 0.221142 \mathrm{~Hz}$

AQ $\quad 2.2609921 \mathrm{sec}$

RG

$\mathrm{DW}$

$4 a i$ 


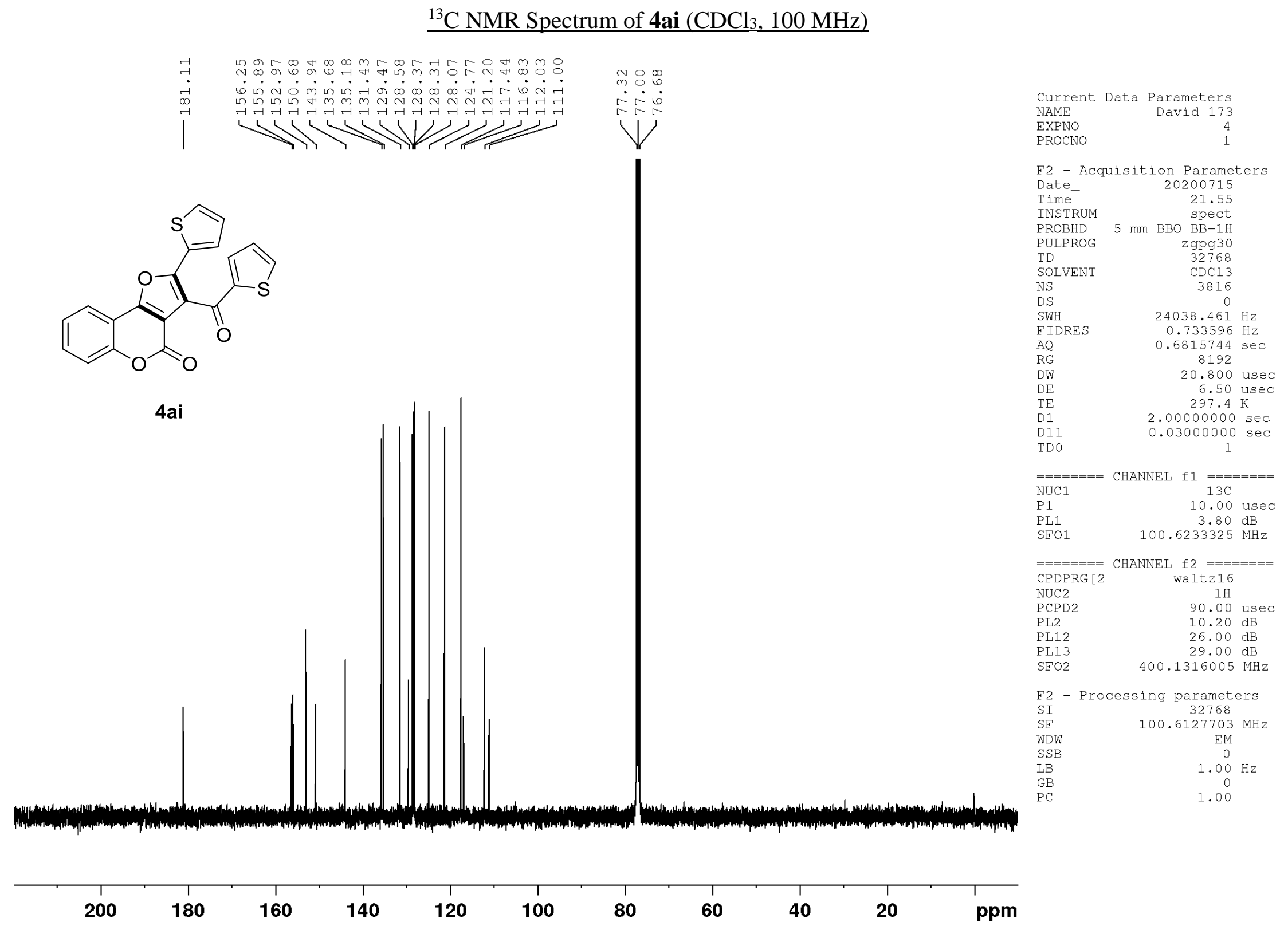


$\underline{{ }^{1} \mathrm{H} \text { NMR Spectrum of } 4 \mathrm{aj}\left(\mathrm{CDCl}_{3}, 400 \mathrm{MHz}\right)}$

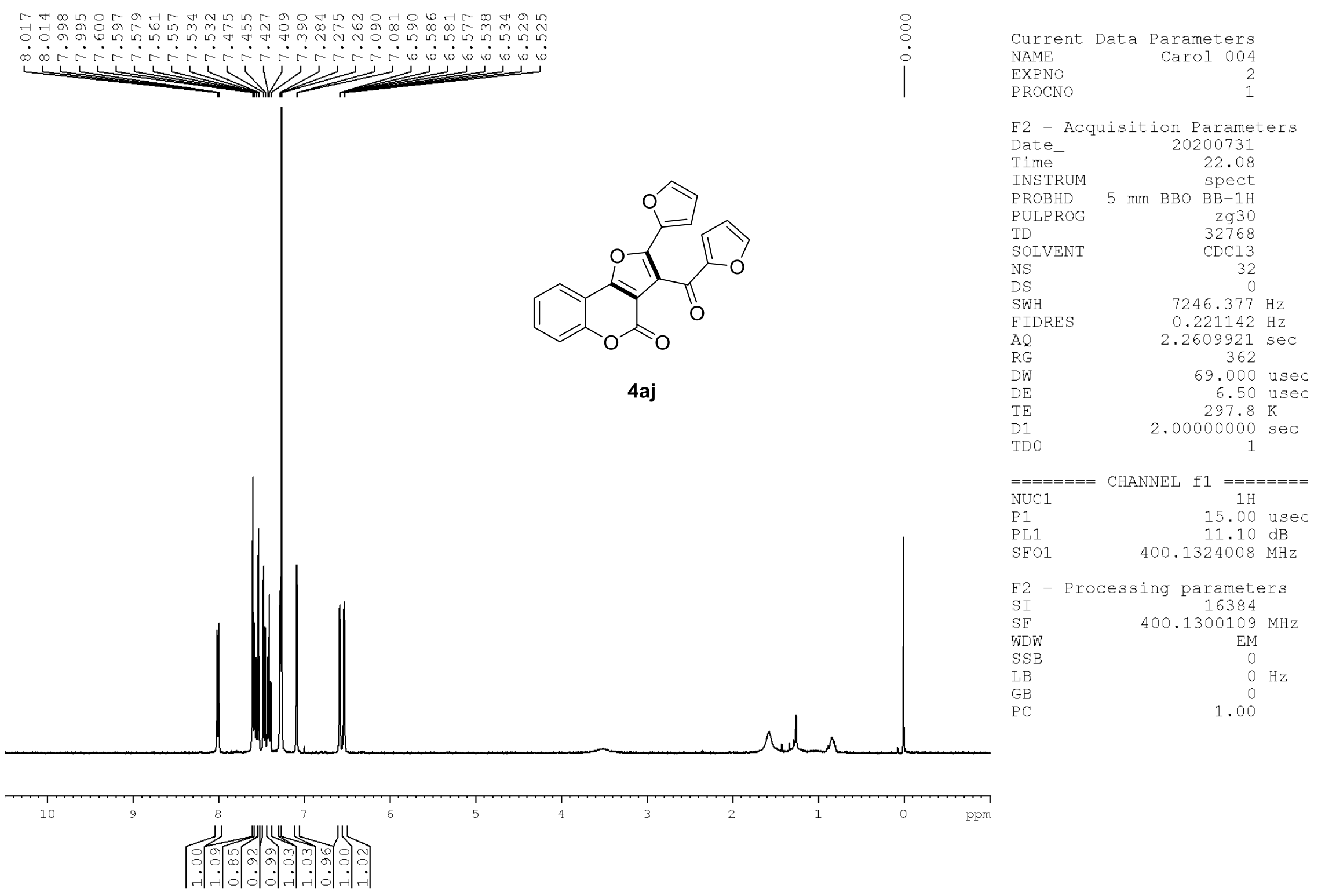




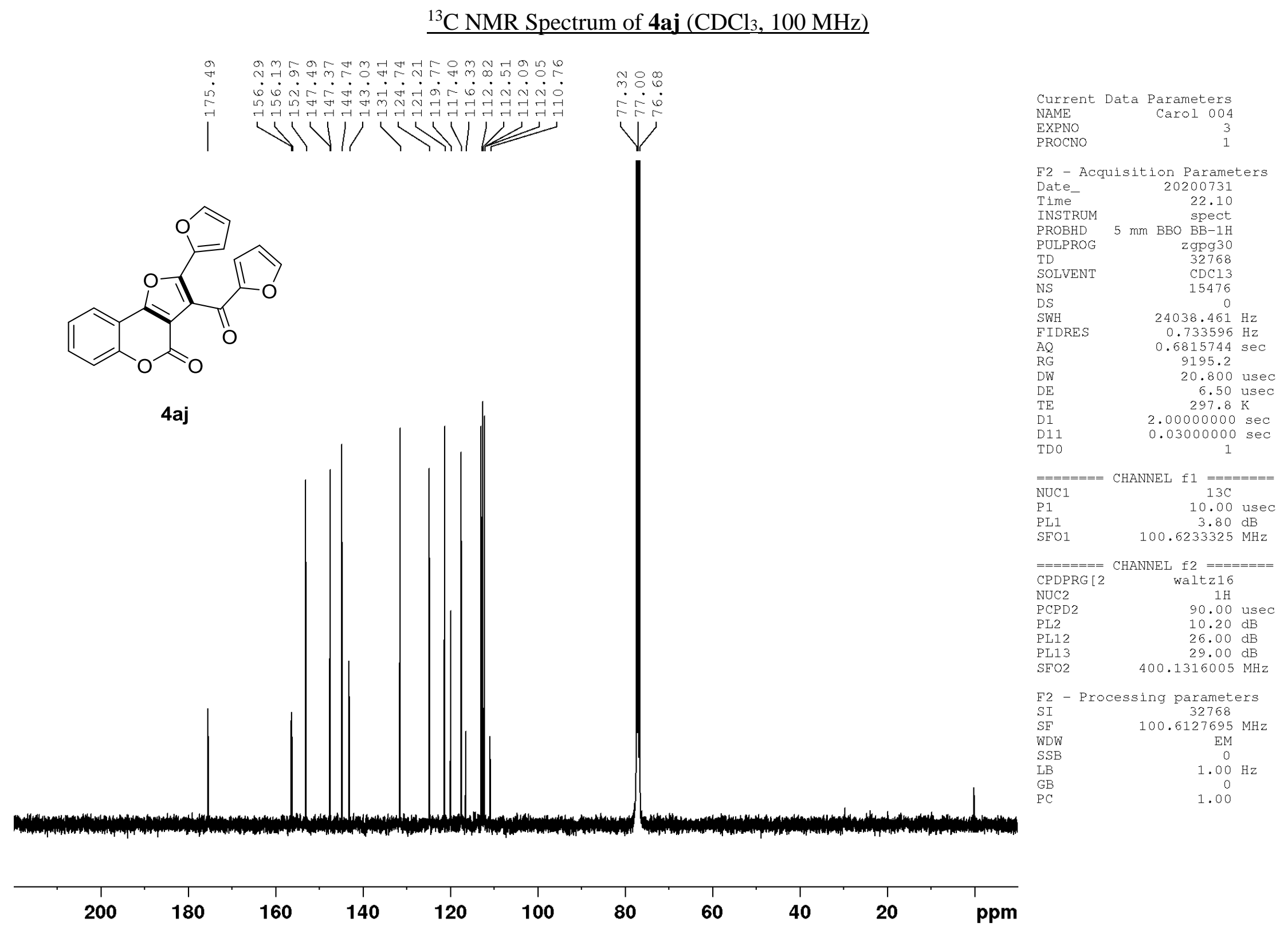


${ }^{1} \mathrm{H}$ NMR Spectrum of $\mathbf{4 a k}\left(\mathrm{CDCl}_{3}, 400 \mathrm{MHz}\right)$

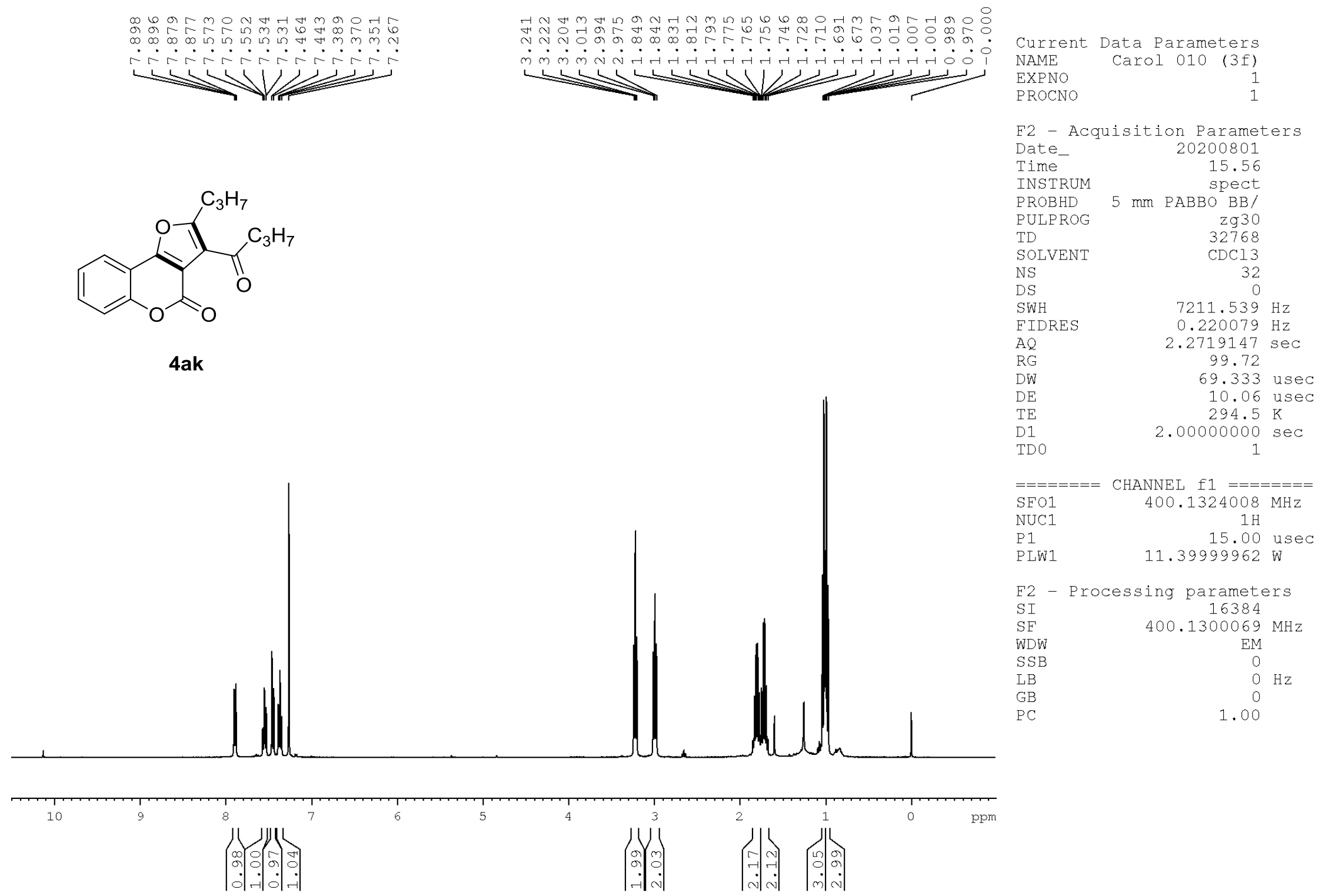


${ }^{13} \mathrm{C}$ NMR Spectrum of 4ak $\left(\mathrm{CDCl}_{3}, 100 \mathrm{MHz}\right)$

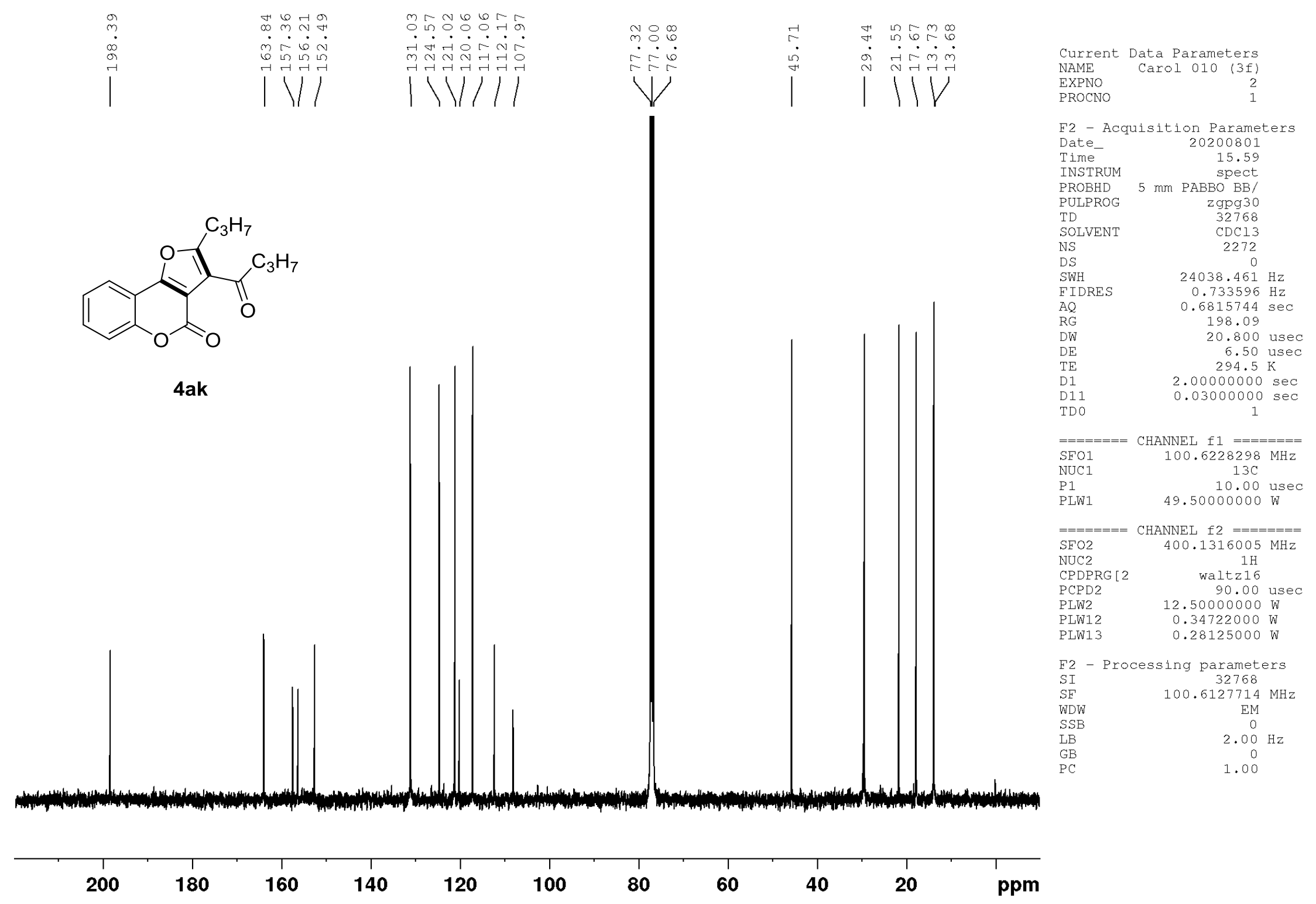


$\underline{{ }^{1} \mathrm{H} \text { NMR Spectrum of } 4 \mathrm{al}\left(\mathrm{CDCl}_{3}, 400 \mathrm{MHz}\right)}$

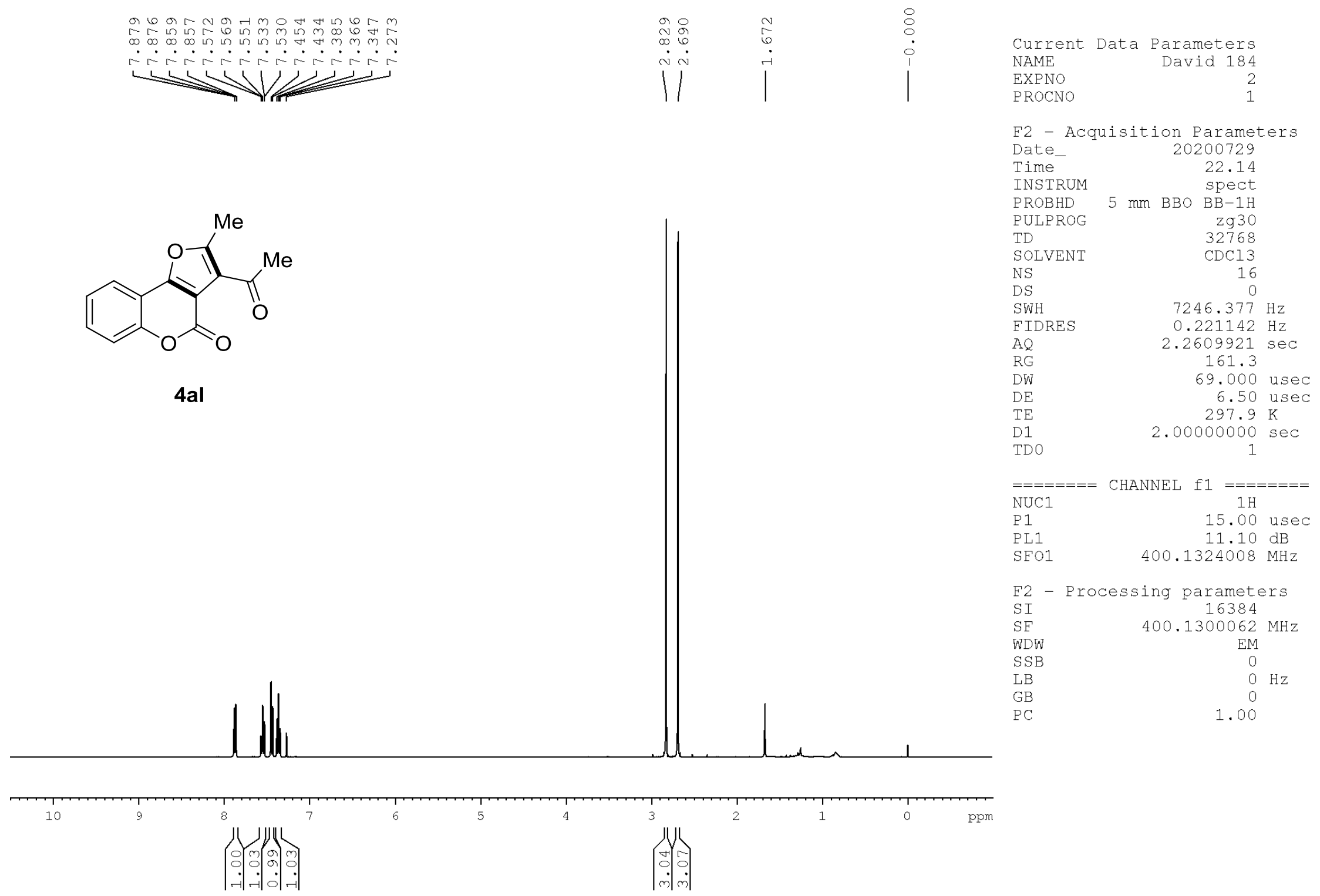


${ }^{13} \mathrm{C}$ NMR Spectrum of $4 \mathrm{al}\left(\mathrm{CDCl}_{3}, 100 \mathrm{MHz}\right)$

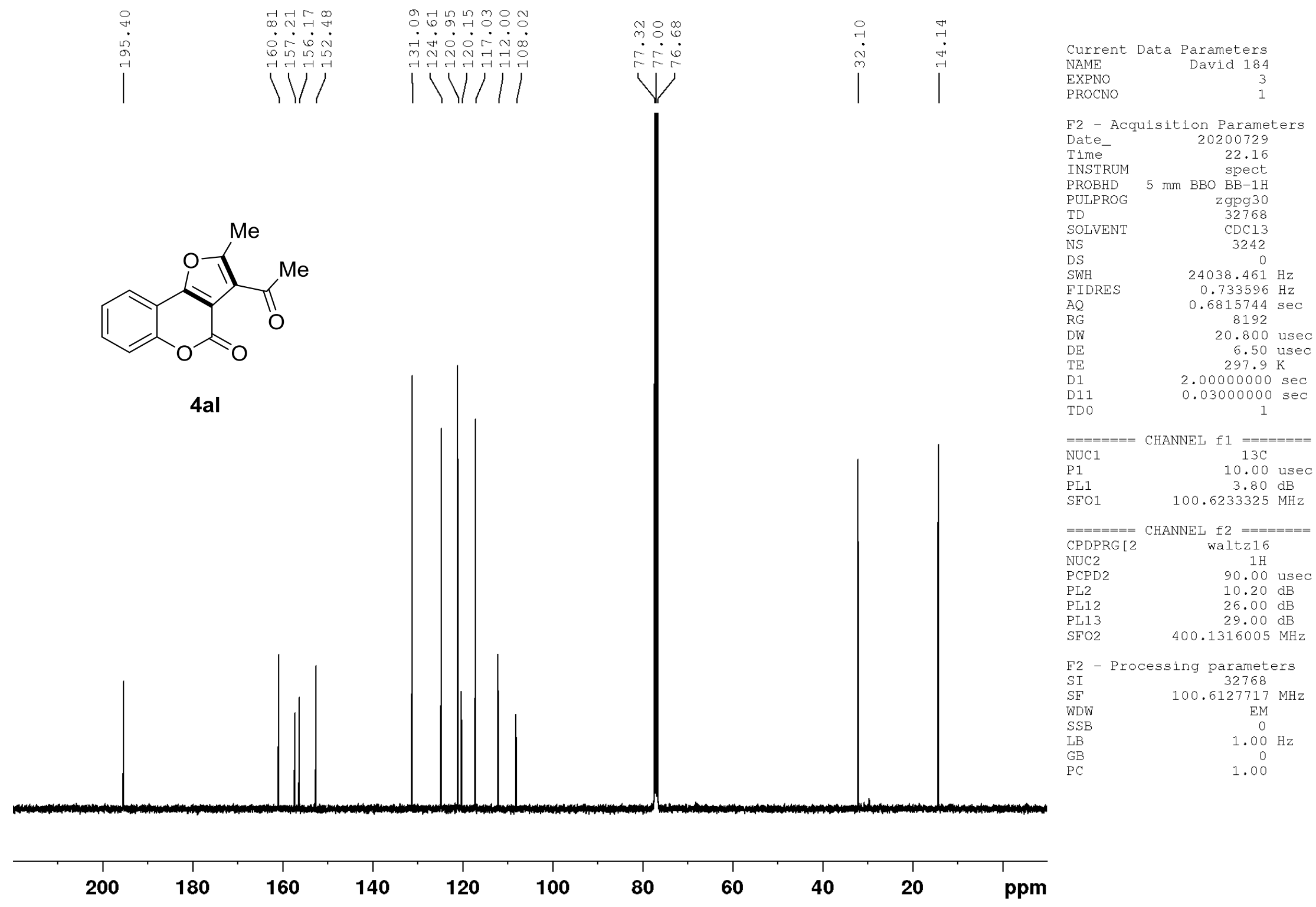


$\underline{{ }^{1} \mathrm{H} \text { NMR Spectrum of } 8\left(\mathrm{CD}_{3} \mathrm{OD}, 400 \mathrm{MHz}\right)}$

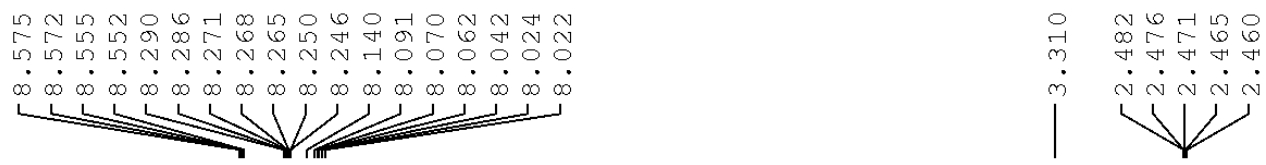

$\begin{array}{lrr}\text { Current Data Parameters } \\ \text { NAME } & \text { Carol } 011 & (3 \mathrm{f}) \\ \text { EXPNO } & & 10 \\ \text { PROCNO } & & 1\end{array}$

F2 - Acquisition Parameters

Date__ 20200908

Time 22.08

INSTRUM spect

PROBHD $5 \mathrm{~mm}$ PABBO BB/

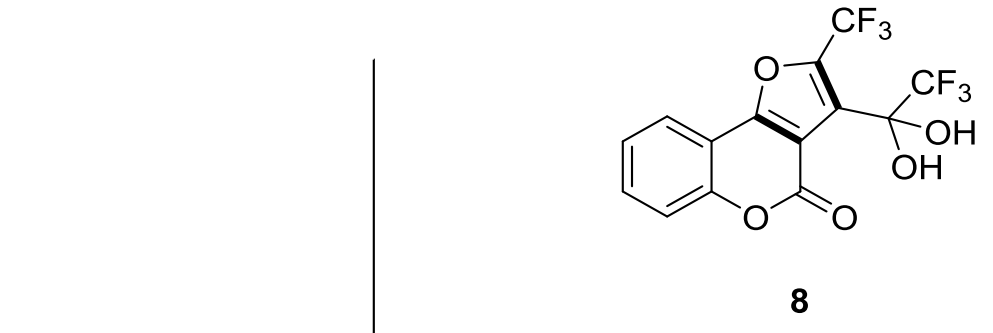

PUI RROG

$\mathrm{zg} 30$

$\mathrm{TD}$

SOLVENT

NS

DS

$\mathrm{SWH}$

FIDRES

$\mathrm{AQ}$
$\mathrm{RG}$

DW

$\mathrm{DE}$

D1

TDO

$=====$

$\mathrm{SEO}$

NUC1

P1

$$
\begin{array}{r}
32768 \\
\text { Acetone }
\end{array}
$$

0
0
7211.539

$0.220079 \mathrm{~Hz}$

$2.2719147 \mathrm{sec}$

198.09

69.333 usec

10.06 usec

$2.00000000 \mathrm{sec}$

CHANNEL $\mathrm{f} 1=======$ 400.1324008 $\mathrm{MHz}$

PLW1

$1 \mathrm{H}$

15.00 usec

$11.39999962 \mathrm{~W}$

F2 - Processing parameters

SI 16384

SF $\quad 400.1298384 \mathrm{MHz}$

WDW

SSB

LB

GB

0

1.00

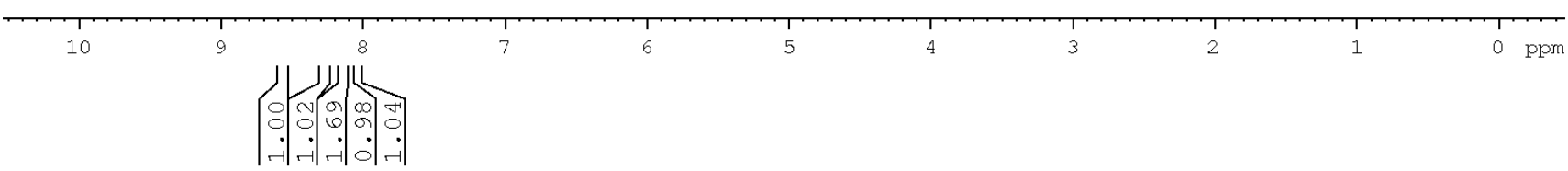


${ }^{13} \mathrm{C}$ NMR Spectrum of $8\left(\mathrm{CD}_{3} \mathrm{OD}, 100 \mathrm{MHz}\right)$
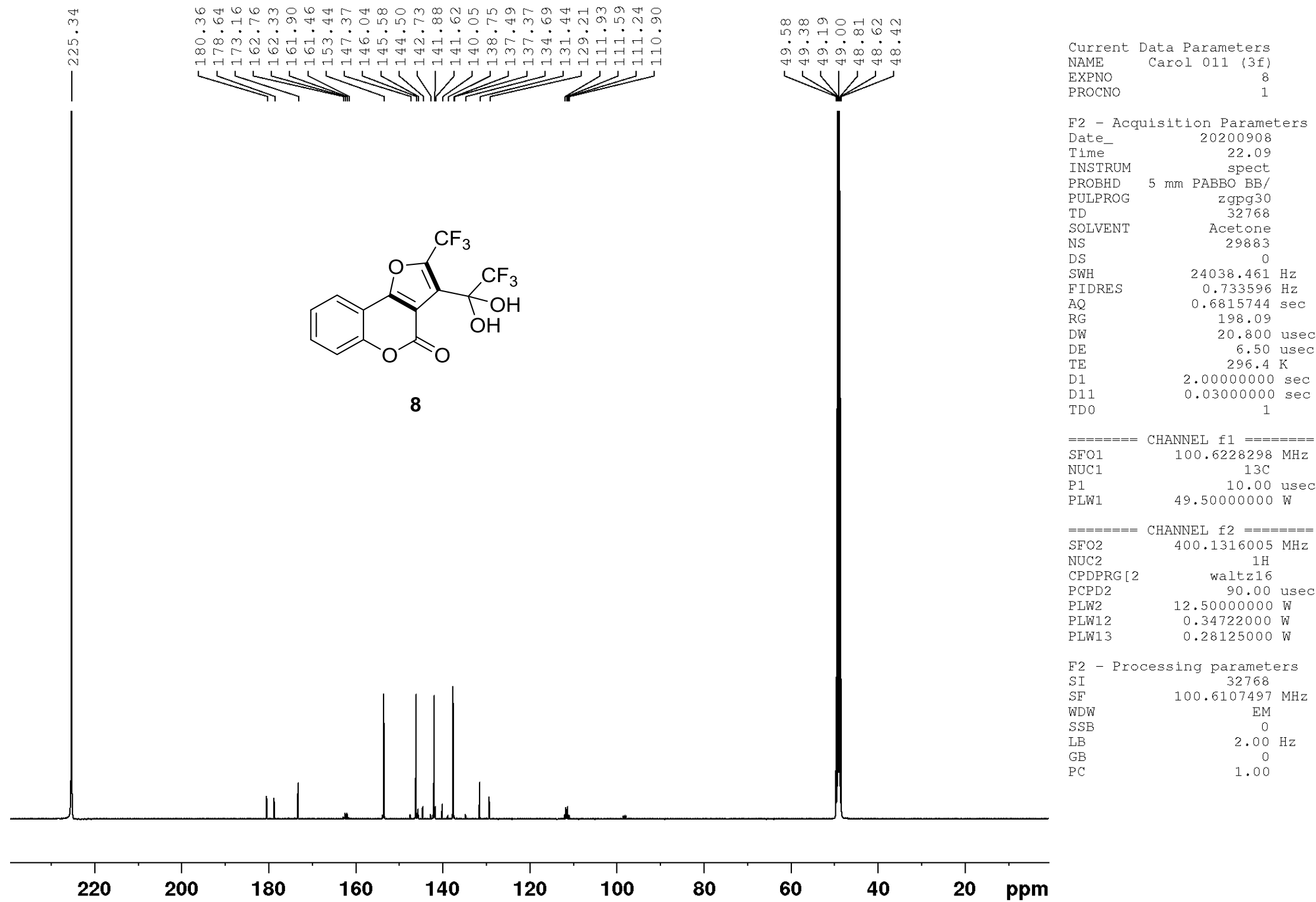

220 
${ }^{19} \mathrm{~F}$ NMR Spectrum of $8\left(\mathrm{CD}_{3} \mathrm{OD}, 376 \mathrm{MHz}\right)$
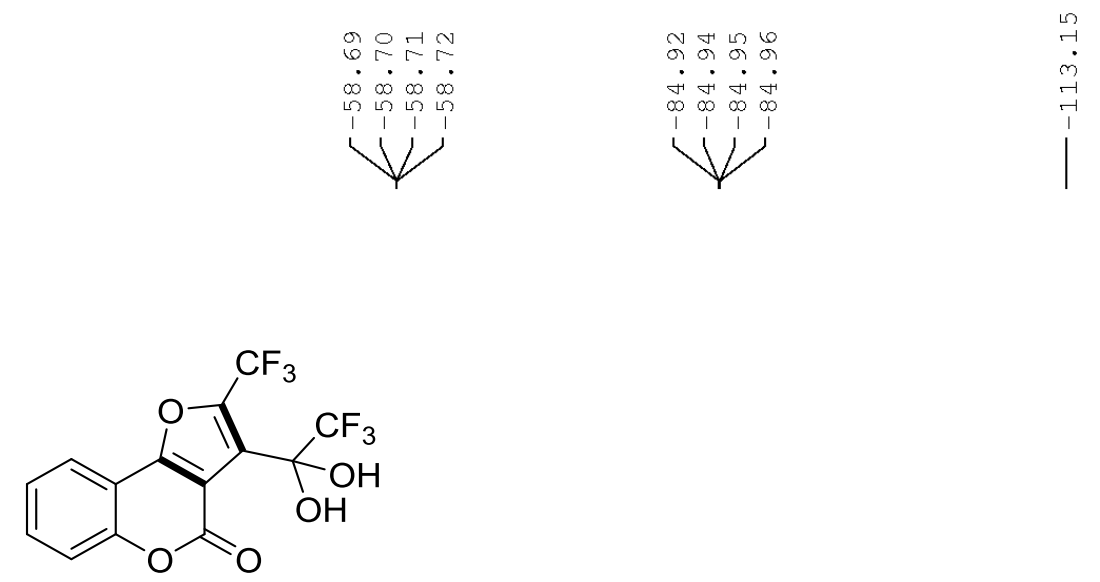

Current Data Parameters NAME Carol 011 (3f) PROCNO

F2 - Acquisition Parameter Date_ratsition 20200915

Time

INSTRUM

PROBHD

20.43
spect

PULPROG 5 m PABBO BB/

TD

DS

FIDRE

$\mathrm{AQ}$

RG

DE

8

TE

D 11

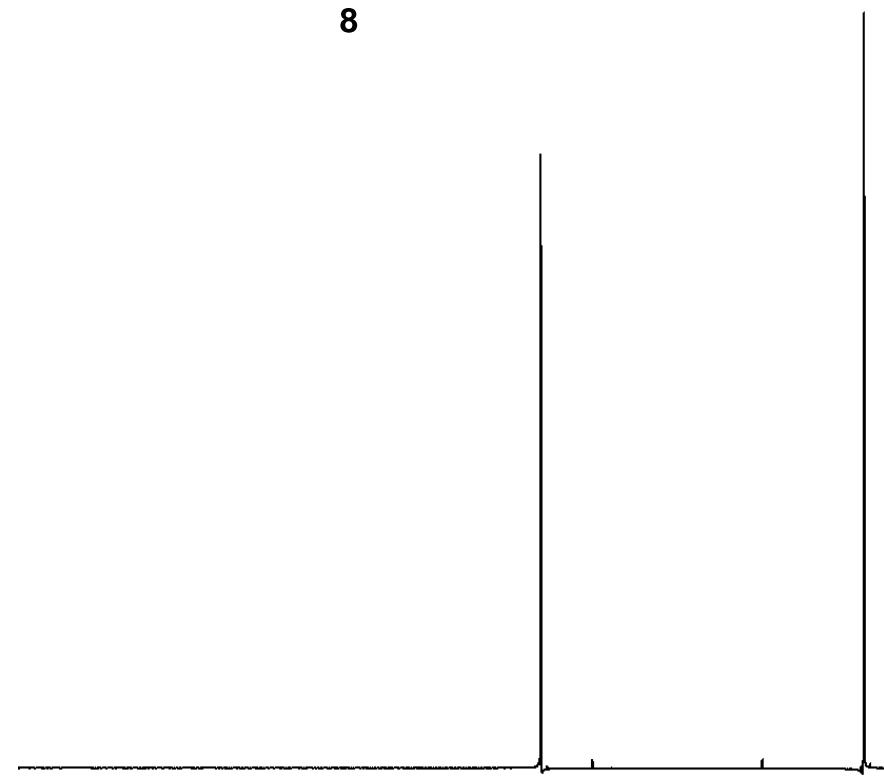

TDO

SFO1

$\begin{array}{lr}\text { SFO1 } & 376.4607168 \mathrm{MHz} \\ \text { NUC1 } & 19 \mathrm{~F} \\ \text { P1 } & 15.00 \text { usec }\end{array}$

PLW1 $18.00000000 \mathrm{~W}$

$====$
$\mathrm{SFO} 2$ CHANNEL $f$

$89285.711 \mathrm{~Hz}$
$0.681196 \mathrm{~Hz}$

$0.681196 \mathrm{~Hz}$ $0.7340032 \mathrm{sec}$
198.09 198.09

6.50 usec

1.00000000 sec

$0.03000000 \mathrm{sec}$

CPDPRG [ 2

PCPD2

PLW 2

$400.1316005 \mathrm{MHz}$

PLW12

waltz16

90.00 use

$0.34722000 \mathrm{~W}$

SI $\quad 376.4980246$

SF $\quad 376.4$

WSB

LB

$\mathrm{PC}$

$\mathrm{EM}$
0
$1.00 \mathrm{~Hz}$
0

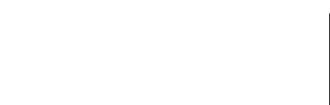

1.0

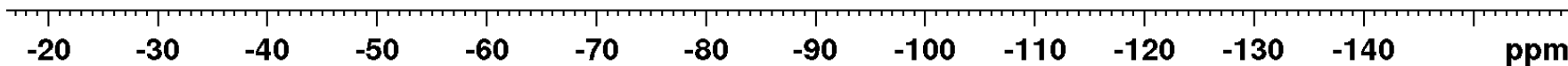


$\underline{{ }^{1} \mathrm{H} \text { NMR Spectrum of } \mathbf{6 a a}\left(\mathrm{CDCl}_{3}, 400 \mathrm{MHz}\right)}$

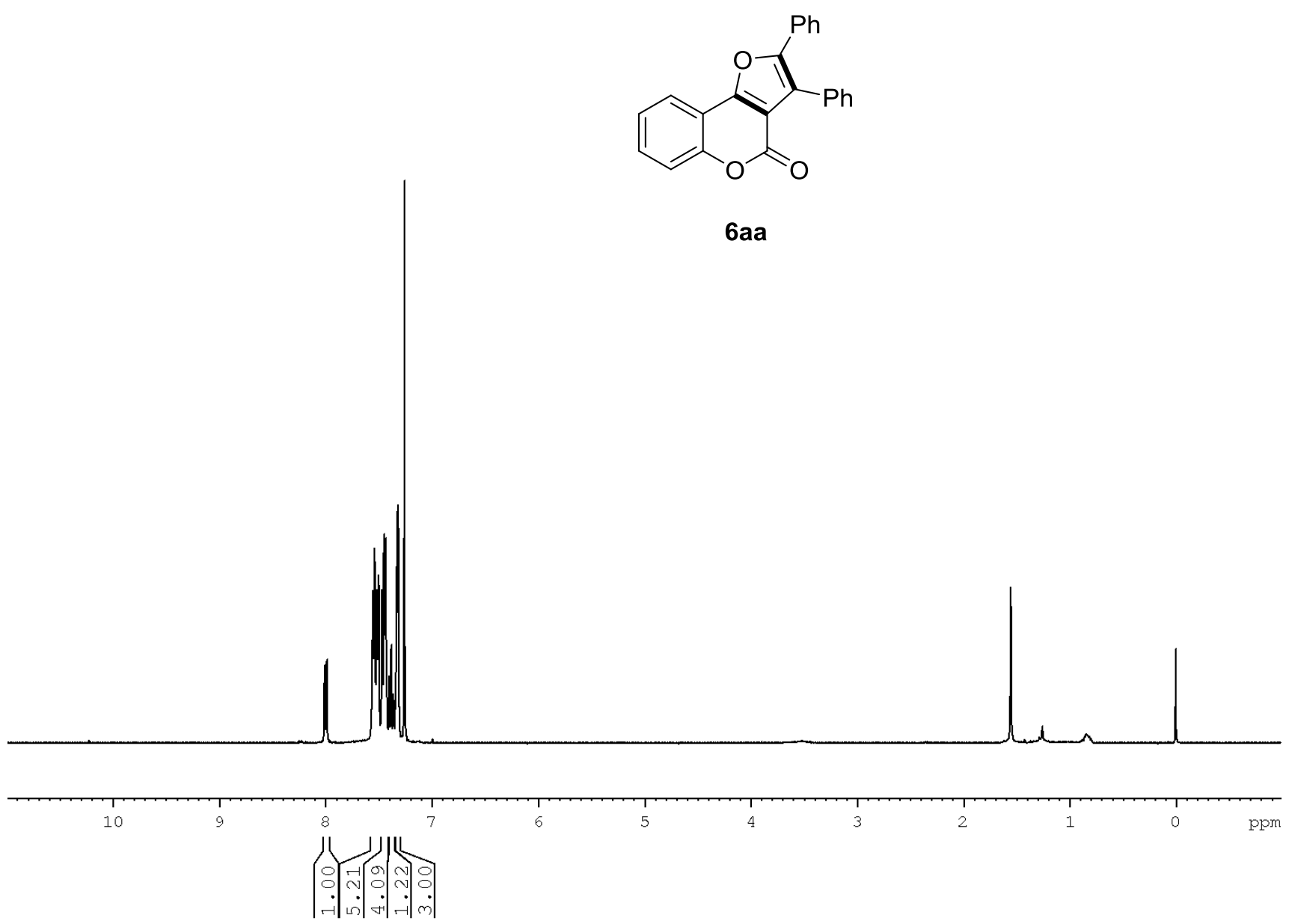

Current Data Parameters NAME

EXPNO

PROCNO

David 183

F2 - Acquisition Parameters

$\begin{array}{lr}\text { Date_ } & 20200804 \\ \text { Time } & 23.12\end{array}$

Time

INSTRUM

PROBHD

PULPROG

$\mathrm{TD}$

SOLVEN

NS

DS

SWH

FIDRES

AQ

$\mathrm{RG}$

DW

$\mathrm{DE}$

D1

TDO

mm BBO spect

$\mathrm{BB}-1 \mathrm{H}$

$\mathrm{zg} 30$

CDC13

16

$7246.377 \mathrm{~Hz}$

$0.221142 \mathrm{~Hz}$

2.2609921 sec

322.5

69.000 usec

6.50 usec

$297.1 \mathrm{~K}$

$2.00000000 \mathrm{sec}$

$\begin{array}{lr}=======\text { CHANNEL } \mathrm{fl}======= \\ \text { NUC1 } & 1 \mathrm{H} \\ \text { P1 } & 15.00 \mathrm{usec} \\ \text { PL1 } & 11.10 \mathrm{~dB} \\ \text { SE01 } & 1324008 \mathrm{mHz}\end{array}$

SFO1 $400.1324008 \mathrm{MH}$

F2 - Processing parameters

$\begin{array}{lr}\text { SI } & 16384 \\ \text { SF } & 400.1300127 \mathrm{MHz}\end{array}$

WDW

SSB

$L B$

$\mathrm{GB}$
$\mathrm{PC}$ 
${ }^{13} \mathrm{C}$ NMR Spectrum of $6 \mathbf{a a}\left(\mathrm{CDCl}_{3}, 100 \mathrm{MHz}\right)$

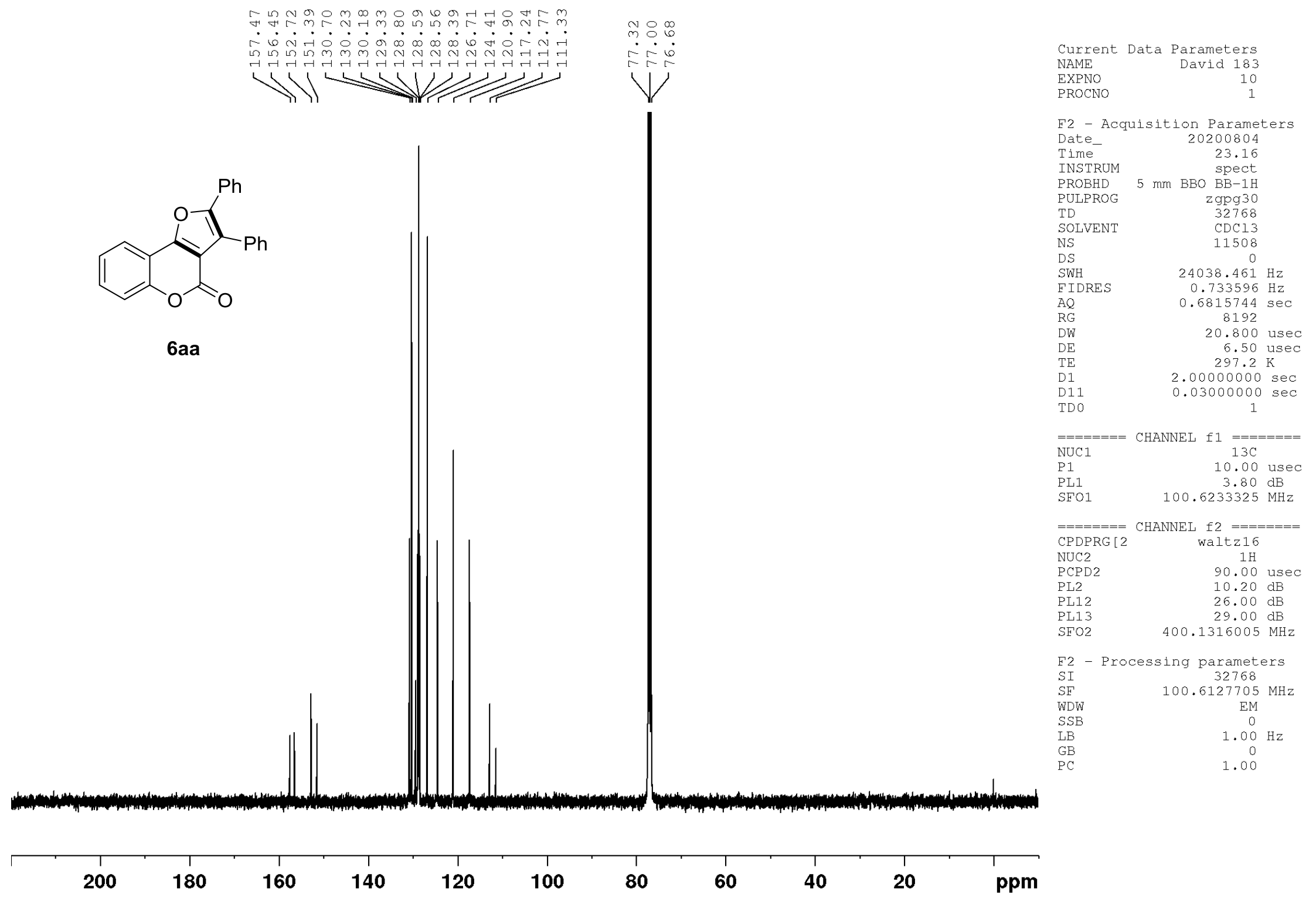


${ }^{1} \mathrm{H}$ NMR Spectrum of $6 \mathbf{b a}\left(\mathrm{CDCl}_{3}, 400 \mathrm{MHz}\right)$
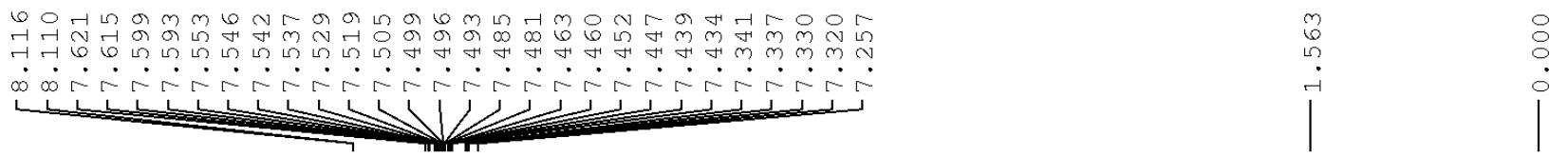

$\begin{array}{lrr}\text { Current } & \text { Data Parameters } \\ \text { NAME } & \text { David } 194 & \text { (3f) } \\ \text { EXPNO } & & 1 \\ \text { PROCNO } & & 1\end{array}$

F2 - Acquisition Parameters

Date__ 20200819

Time 11.32

INSTRUM spect

PROBHD $5 \mathrm{~mm}$ PABBO BB/

PULPROG $\quad$ zg30

$\begin{array}{lr}\text { PG } & \mathrm{Zg} 30 \\ \mathrm{TD} & 32768\end{array}$

$\mathrm{TD}$

SOLVENT

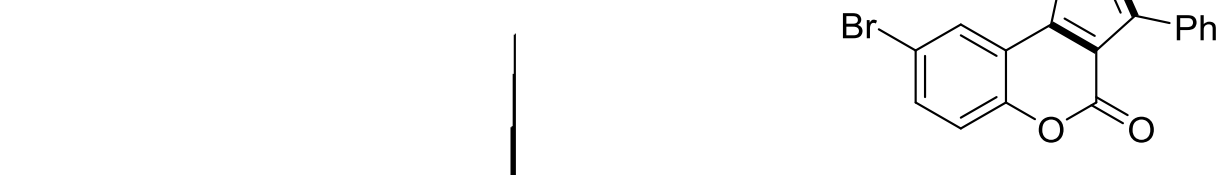

$6 \mathrm{ba}$

DS

SWH

FIDRES

$\mathrm{AQ}$
$\mathrm{RG}$
$\mathrm{DWT}$

DW

DE

TE 1

TD0

$====$
SFO1

$====$
SEO1
NUC1

$\mathrm{P} 1$

PLW1

$\mathrm{CDCl} 3$

0
$7211.539 \mathrm{~Hz}$
$0.220079 \mathrm{~Hz}$ $2.2719147 \mathrm{sec}$ 198.09

69.333 usec

10.06 usec

$297.5 \mathrm{~K}$

$2.00000000 \mathrm{sec}$ 1

$400.1324008 \mathrm{MHz}$ 15.00 usec

$11.39999962 \mathrm{~W}$

F2 - Processing parameters

SI 16384

SF $\quad 400.1300108 \mathrm{MHz}$

WDW

SSB

LB

GB

400.1300108

EM
0
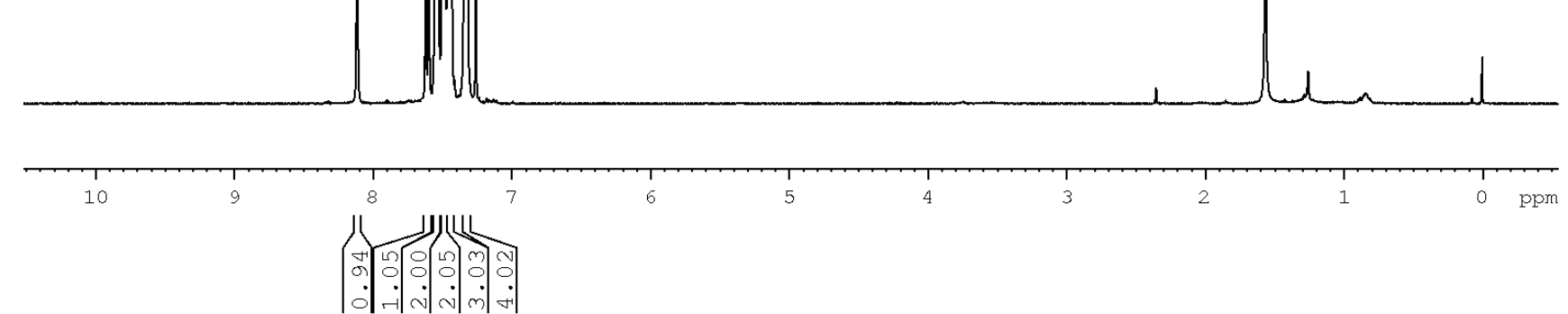
${ }^{13} \mathrm{C}$ NMR Spectrum of $6 \mathbf{b a}\left(\mathrm{CDCl}_{3}, 100 \mathrm{MHz}\right)$

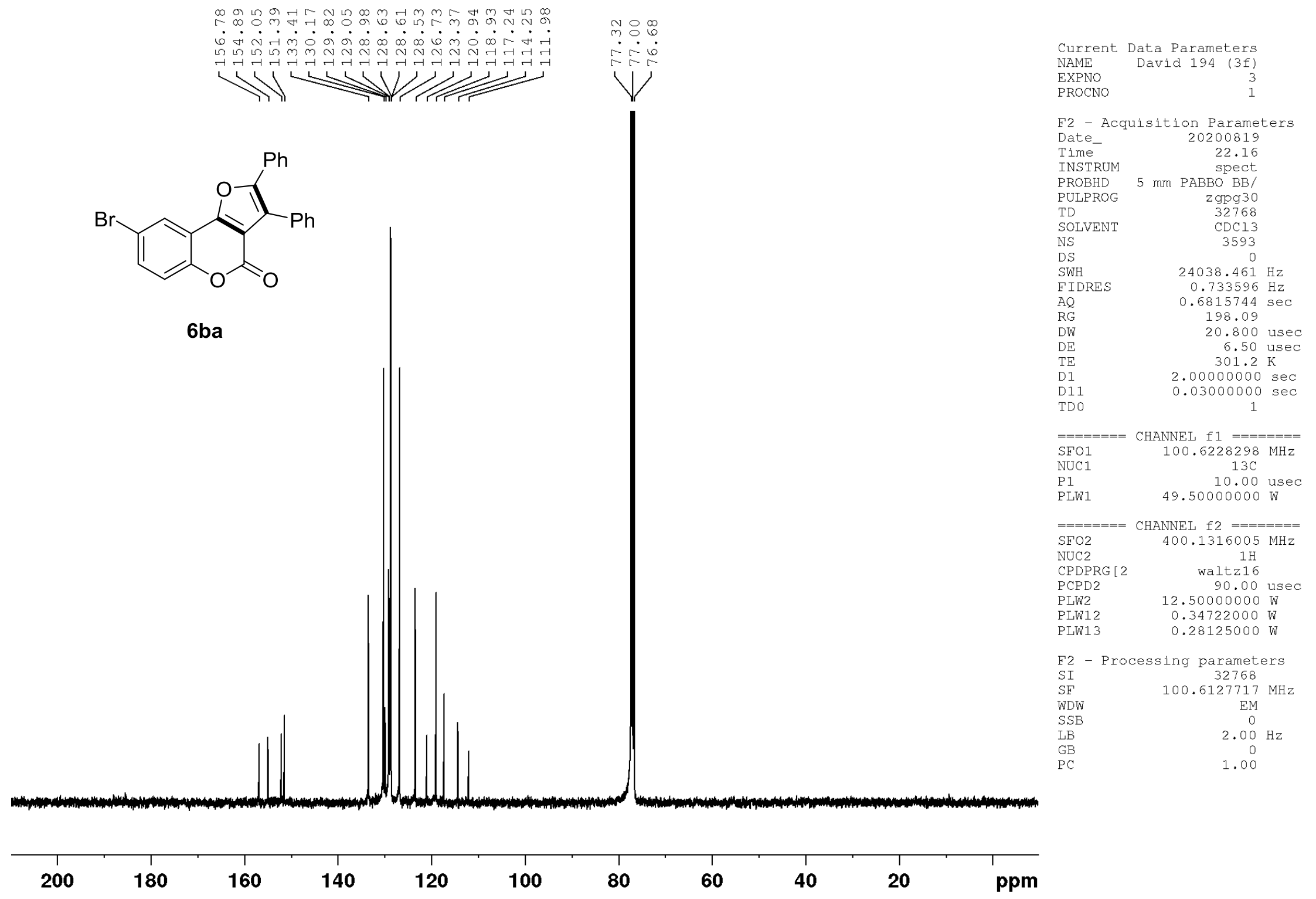


$\underline{{ }^{1} \mathrm{H} \text { NMR Spectrum of } \mathbf{6 c a}\left(\mathrm{CDCl}_{3}, 400 \mathrm{MHz}\right)}$

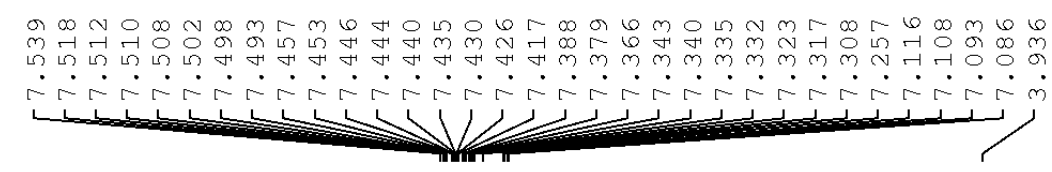

Current Data Parameters

NAME

EXPNO

1

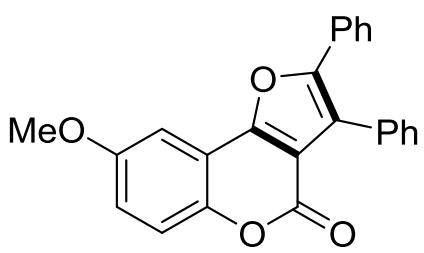

$6 c a$

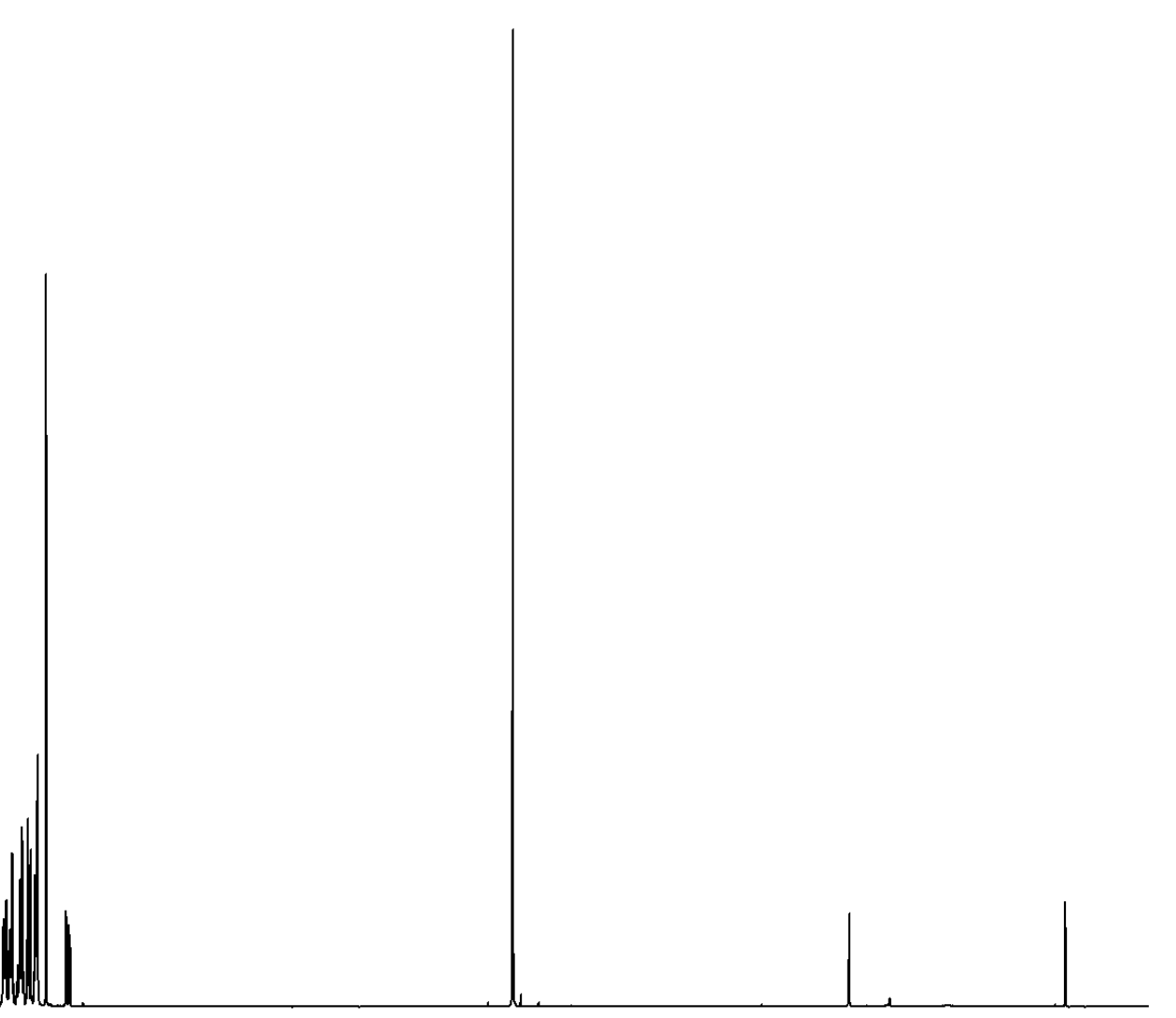

F2 - Acquisition Parameters

Date_ 20200820

Time 1.05

INSTRUM spect

PROBHD $5 \mathrm{~mm}$ PABBO BB/

PULPROG Zg30

TD 32768

SOLVENT CDCl3

NS

DS

SWH $\quad 7211.539 \mathrm{~Hz}$

ETDRES $\quad 0.220079 \mathrm{~Hz}$

AQ $\quad 2.2719147 \mathrm{sec}$

$\begin{array}{lr}\text { RG } & 198.09\end{array}$

DW $\quad 69.333$ usec

$\mathrm{DE} \quad 10.06$ use

$298.8 \mathrm{~K}$

D1 $\quad 2.00000000 \mathrm{sec}$

$===$

SFO1

NUC1

CHANNEL f1 $========$

$1 \mathrm{H}$

PLW1 $11.39999962 \mathrm{~W}$

15.00 usec

F2 - Processing parameters

SI 16384

SF $\quad 400.1300110 \mathrm{MHz}$

WDW EM

SSB

$\mathrm{LB}$
$\mathrm{GB}$

$\mathrm{PC}$

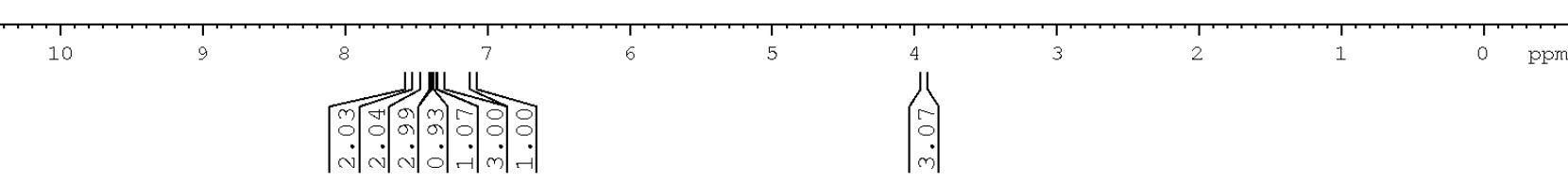


${ }^{13} \mathrm{C}$ NMR Spectrum of $\mathbf{6 c a}\left(\mathrm{CDCl}_{3}, 100 \mathrm{MHz}\right)$

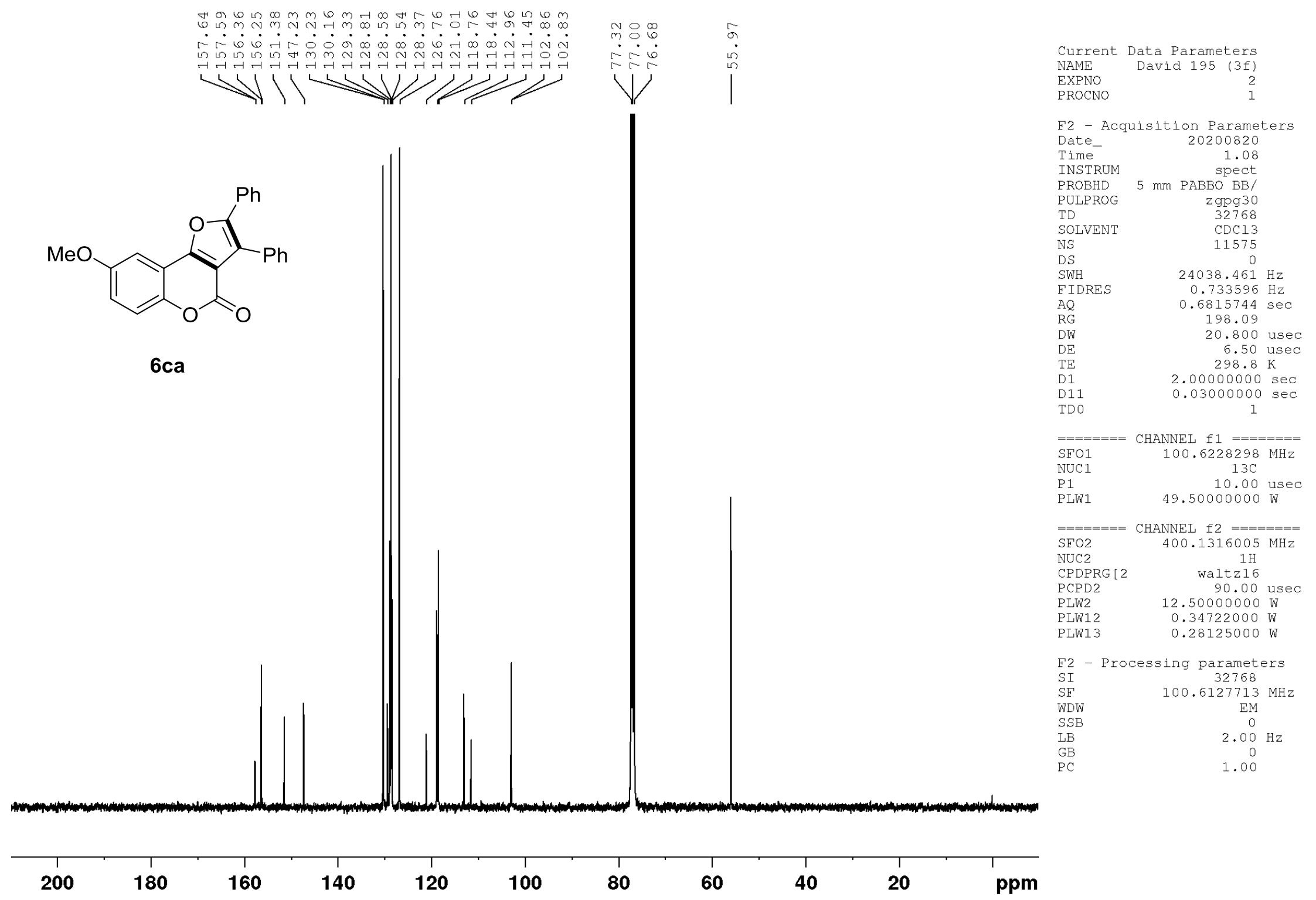


${ }^{1} \mathrm{H}$ NMR Spectrum of $\mathbf{6 a c}\left(\mathrm{CDCl}_{3}, 400 \mathrm{MHz}\right)$
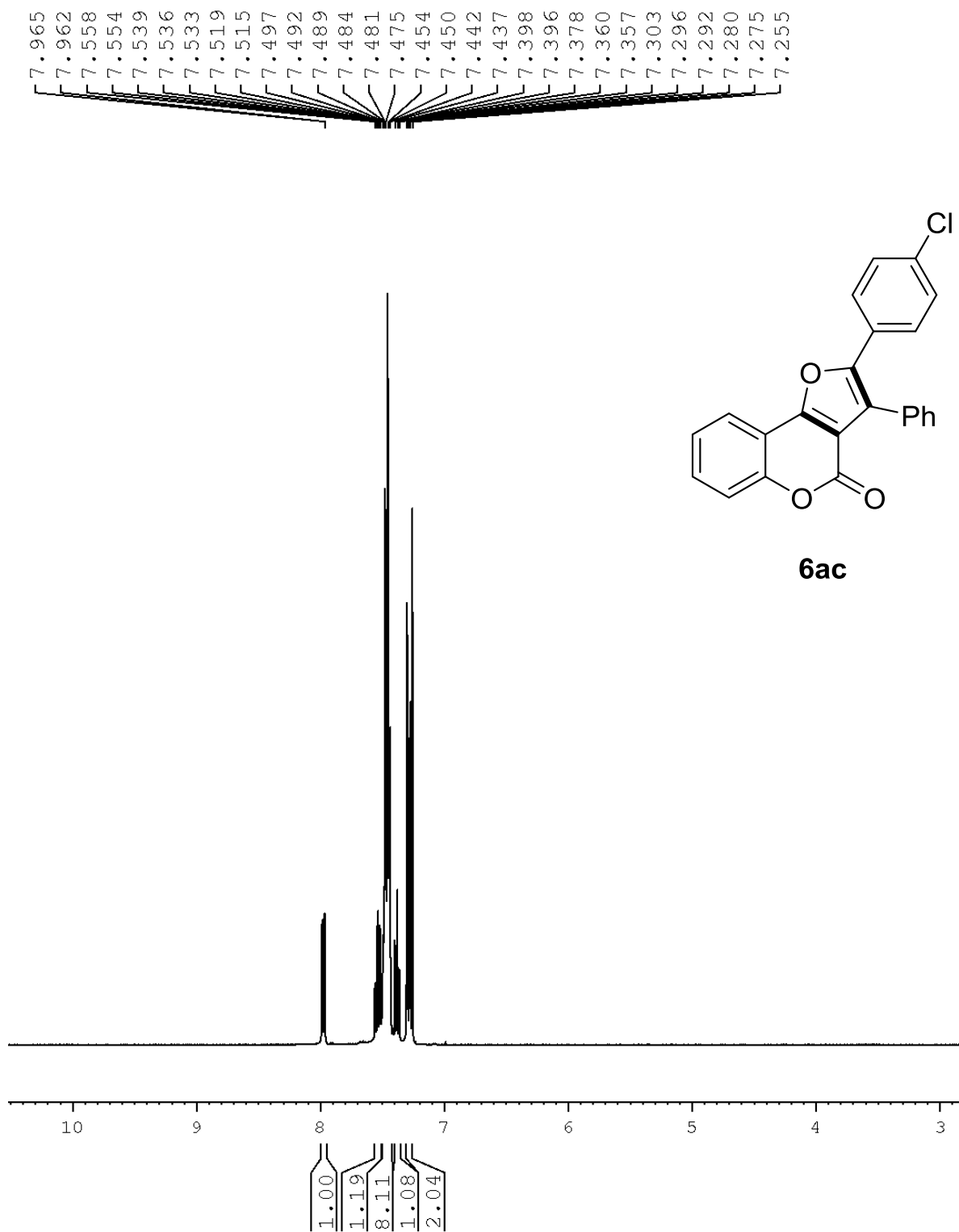

Current Data Parameters NAME Carol 020 (3f) NAME EXPNO PROCNO $\begin{array}{rr}\text { Carol } 020 & (3 f) \\ & 1 \\ & 1\end{array}$

F2 - Acquisition Parameters

Date_ $\quad 20200820$ 21.47

INSTRUM

PROBHD

PULPROG

TD

SOLVENT

NS

DS

$\mathrm{SWH}$

FIDRES

$\mathrm{AQ}$
$\mathrm{RG}$

DW

DE

D1

TDO

$=====$
SEO1

NUCI

$\mathrm{P} 1$

PLW1 $5 \mathrm{~mm} \mathrm{PABBO} \mathrm{BB} /$ $\mathrm{zg} 30$ $\mathrm{zg} 30$
32768 $\mathrm{CDCl} 3$ 0
$7211.539 \mathrm{~Hz}$ $0.220079 \mathrm{~Hz}$ $2.2719147 \mathrm{sec}$ 158.74

69.333 usec

1.033 use

10.06 us

$2.00000000 \mathrm{sec}$

CHANNEL f1 1

F2 - Processing parameters

$\begin{array}{lc}\text { SI } & 16384 \\ \text { SF } & 400.1300118 \mathrm{MHz}\end{array}$

WDW

SSB

$\mathrm{GB}$
$\mathrm{PC}$ 
${ }^{13} \mathrm{C}$ NMR Spectrum of $6 \mathrm{ac}\left(\mathrm{CDCl}_{3}, 100 \mathrm{MHz}\right)$

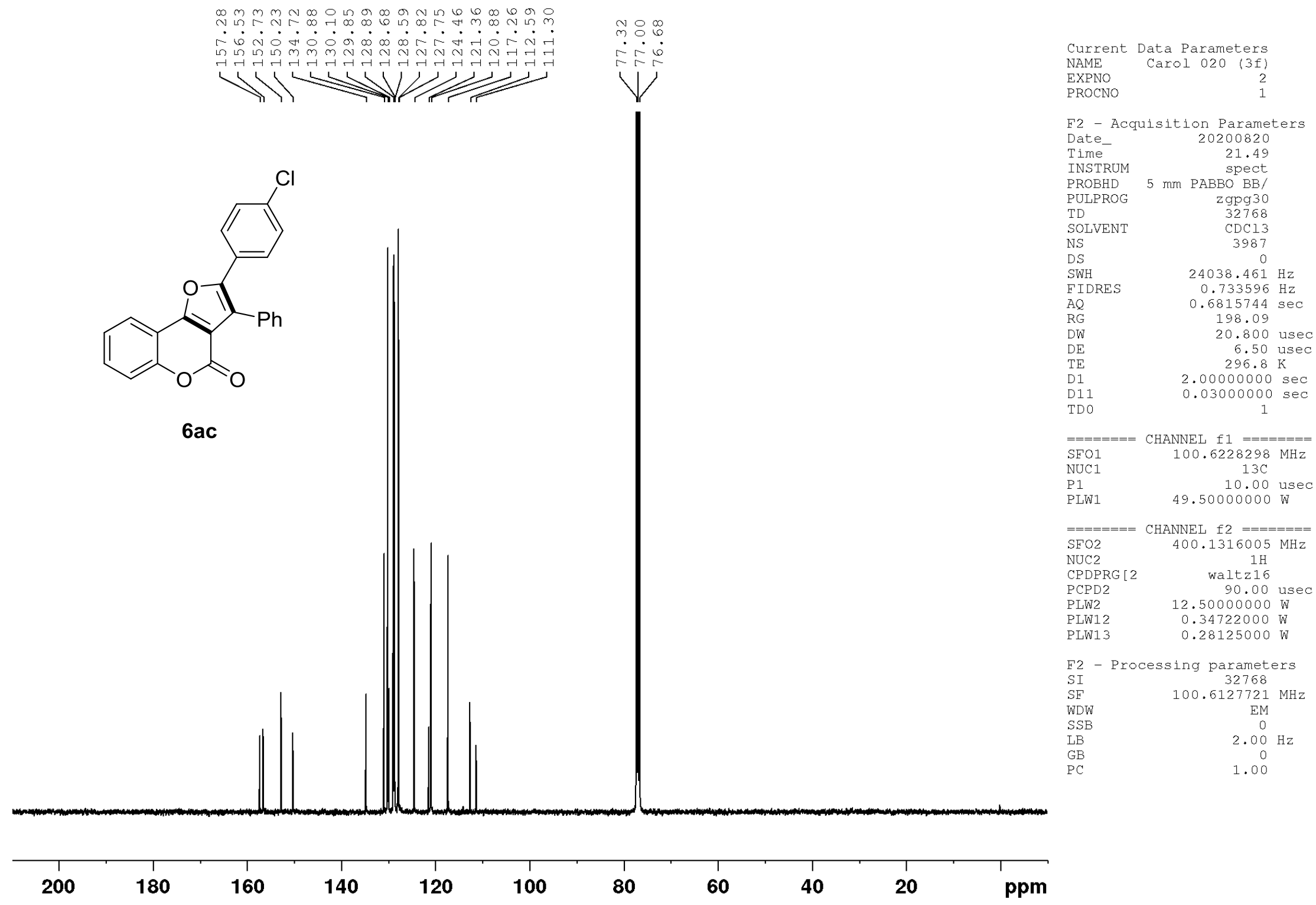


$\underline{{ }^{1} \mathrm{H} \text { NMR Spectrum of } 6 \mathrm{ae}\left(\mathrm{CDCl}_{3}, 400 \mathrm{MHz}\right)}$

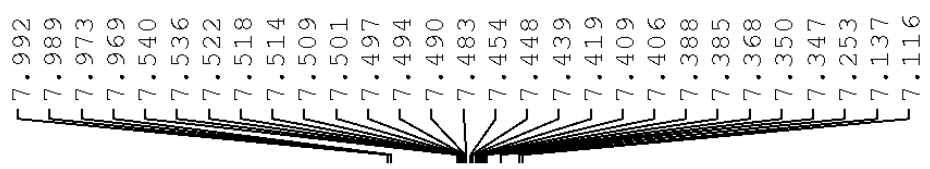

Current Data Parameters

NAME David 196 (3f) EXPNO PROCNO

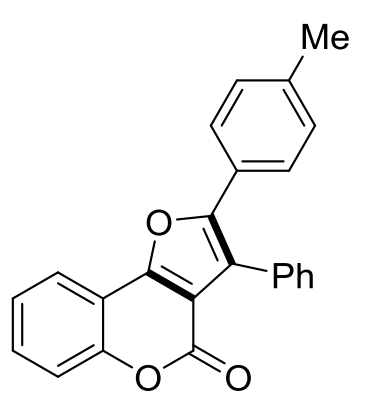

6ae
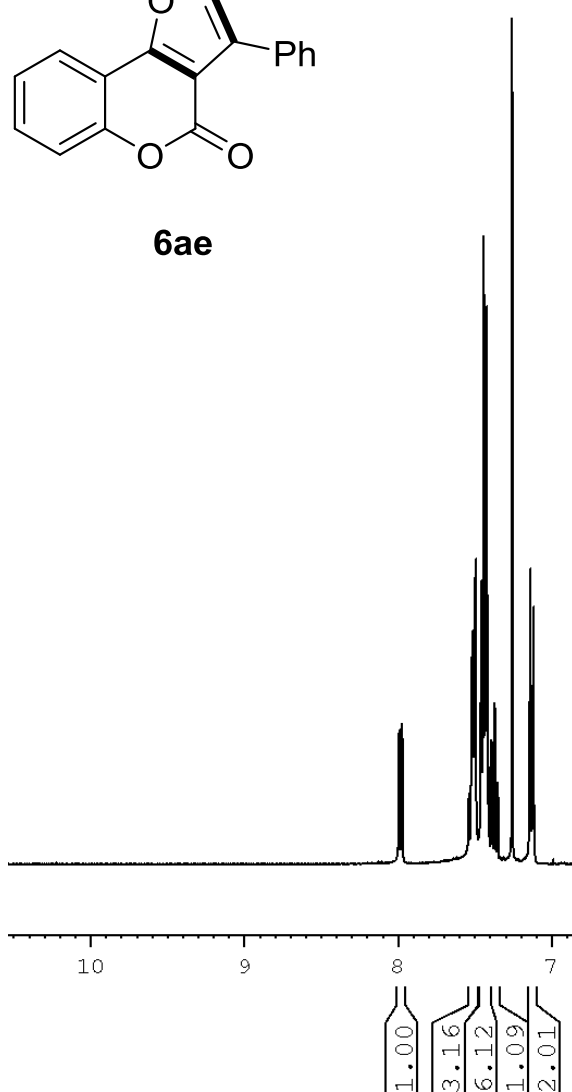

F2 - Acquisition Parameters

Date__ 20200821

Time

INSTRUM

PROBHD

PULPROG

TD

SOLVENT

NS

DS

SWH

FIDRES

$\mathrm{AQ}$
$\mathrm{RG}$

DW

DE

D1

TDO

$======$

$\mathrm{SEO}$

NUC1

P1

0.57

$5 \mathrm{~mm}$ spect

$\mathrm{BB} /$

zg30

32

0
$7211.539 \mathrm{~Hz}$
$0.220079 \mathrm{~Hz}$

2.2719147 sec

177.16

69.333 usec

10.06 usec

298.7

sec

CHANNEL $\mathrm{f} 1=======$ $400.1324008 \mathrm{MHz}$ $1 \mathrm{H}$

PLW1 11.39999962 W

F2 - Processing parameters

SI Processing parameters 16384

SF $\quad 400.1300123 \mathrm{MHz}$

$S E$

WDW
SSB
LB

GB

$\mathrm{PC}$

0

$\mathrm{O} \mathrm{Hz}$

1.00 
${ }^{13} \mathrm{C}$ NMR Spectrum of $6 \mathrm{ae}\left(\mathrm{CDCl}_{3}, 100 \mathrm{MHz}\right)$

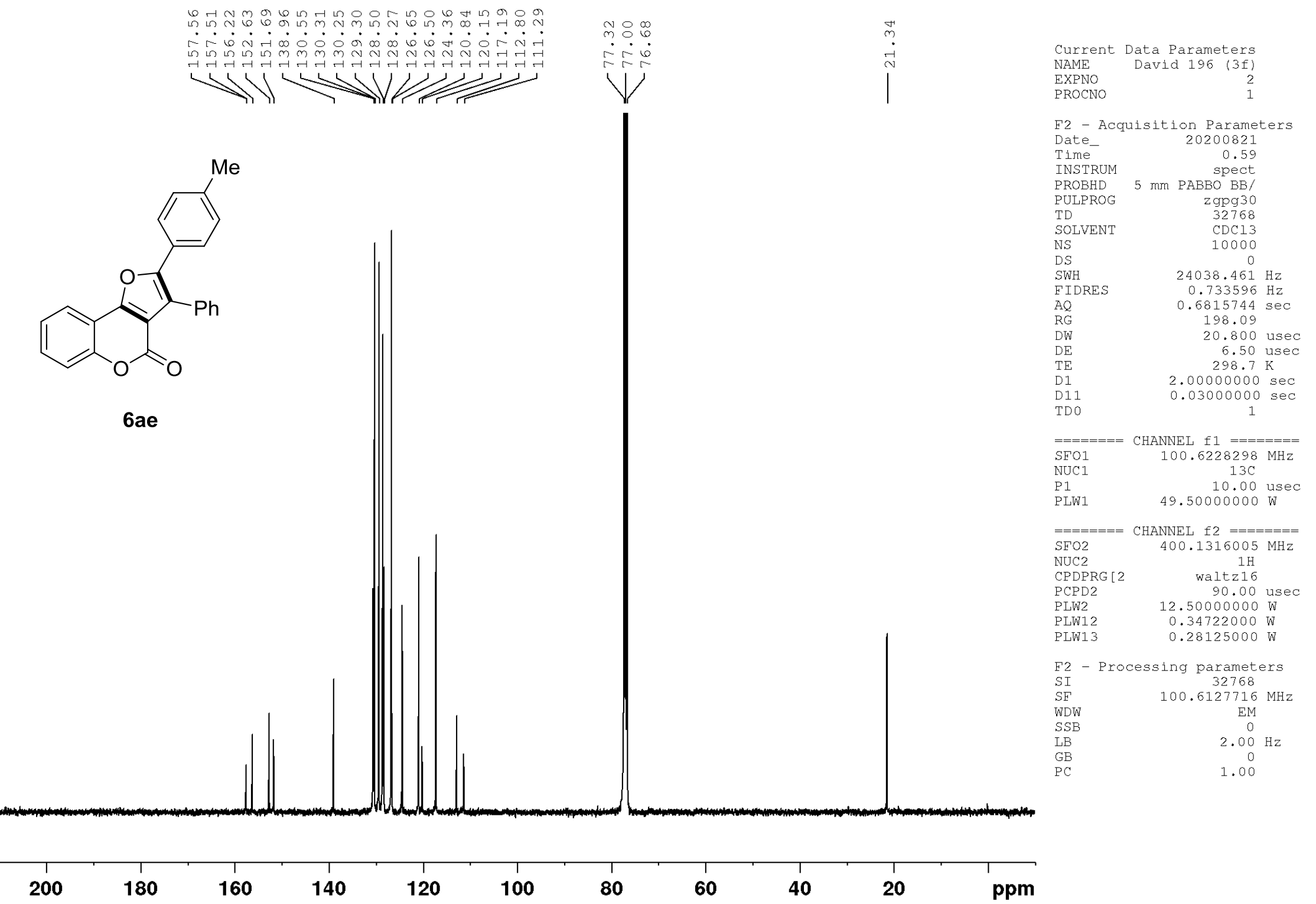


$\underline{{ }^{1} \mathrm{H} \text { NMR Spectrum of } 6 \mathrm{aj}\left(\mathrm{CDCl}_{3}, 400 \mathrm{MHz}\right)}$

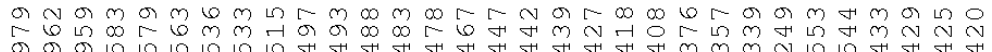

$\therefore \cdots$

r

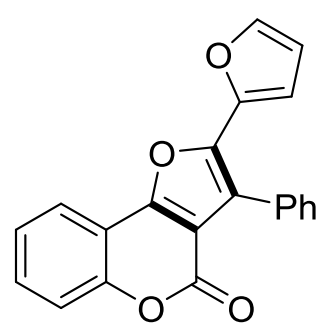

6aj

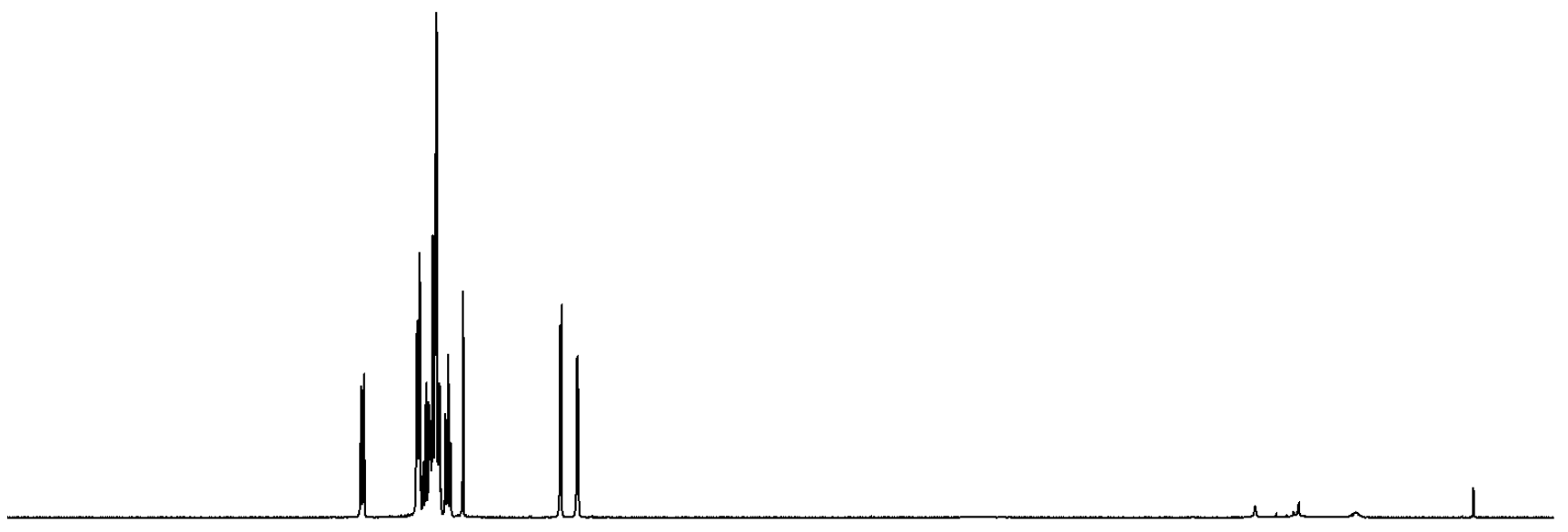

Current Data Parameters NAME

EXPNO
PROCNO Carol 021

F2 - Acquisition Parameters

Date_ $\quad 20200821$

Time

INSTRUM spect

INSTRUM

ROBHD

$\mathrm{mm}$ BBO $\mathrm{BB}-1 \mathrm{H}$

SOLVENT

NS

DS

SWH

FIDRES

$A Q$
$R G$

RG

DW

DE

D1

TDO

$=======$ CHANNEL $\mathrm{f}$

NUC

P1

PL1

$\mathrm{BB}-1 \mathrm{H}$
$\mathrm{zg} 30$

$\mathrm{zg} 30$
32768

0
$7246.377 \mathrm{~Hz}$

$0.221142 \mathrm{~Hz}$ $2.2609921 \mathrm{sec}$$$
114
$$

69.000 usec

6.50 usec

$298.0 \mathrm{~K}$

$2.00000000 \mathrm{sec}$

$400.1324008 \mathrm{MHz}$

F2 - Processing parameters

SI 16384

SF $\quad 400.1300155 \mathrm{MHz}$

WDW

SSB
LB

LB

$\mathrm{PC}$

$\mathrm{EM}$

0
.00

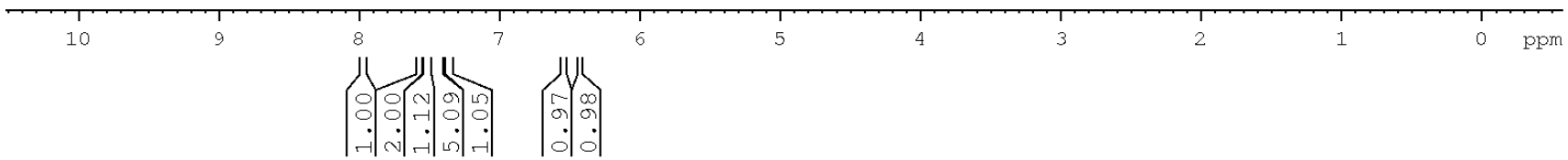


${ }^{13} \mathrm{C}$ NMR Spectrum of $6 \mathrm{aj}\left(\mathrm{CDCl}_{3}, 100 \mathrm{MHz}\right)$

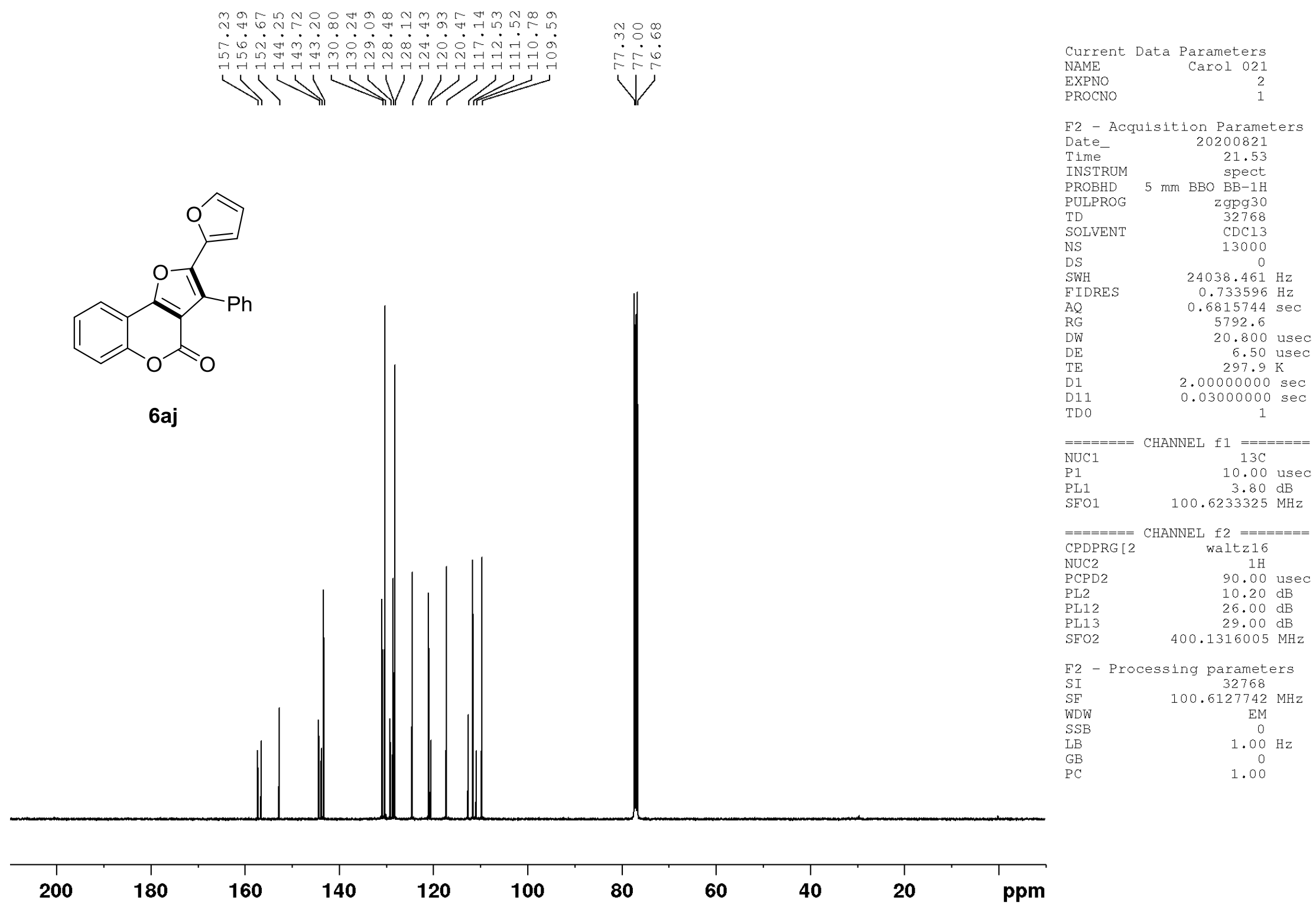


$\underline{{ }^{1} \mathrm{H} \text { NMR Spectrum of } 6 a \mathrm{l}\left(\mathrm{CDCl}_{3}, 400 \mathrm{MHz}\right)}$

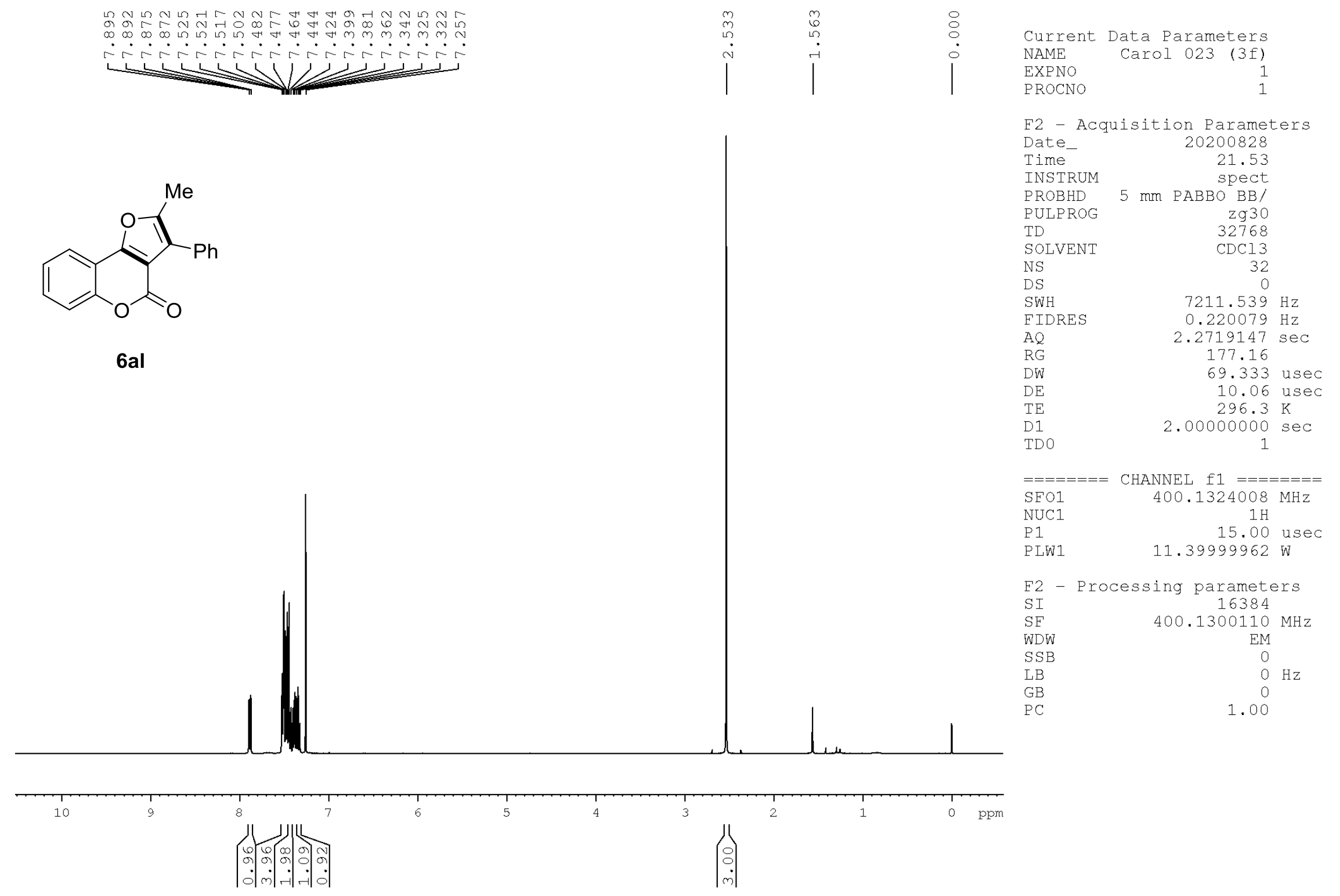


${ }^{13} \mathrm{C}$ NMR Spectrum of $6 \mathrm{al}\left(\mathrm{CDCl}_{3}, 100 \mathrm{MHz}\right)$

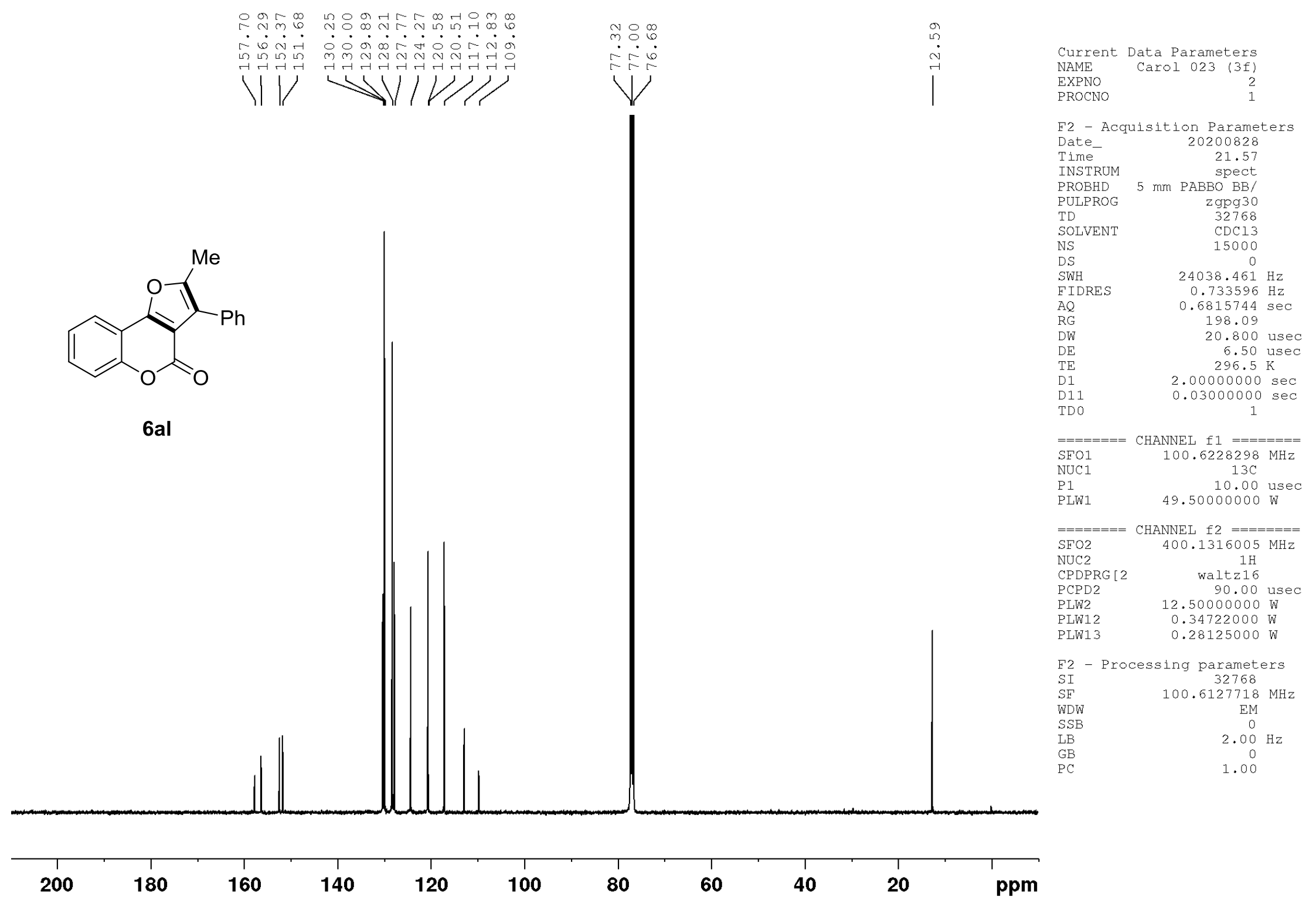


${ }^{1} \mathrm{H}$ NMR Spectrum of $6 \mathbf{a m}\left(\mathrm{CDCl}_{3}, 400 \mathrm{MHz}\right)$

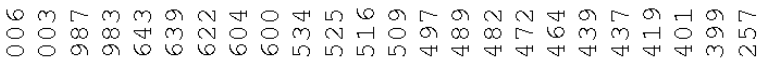

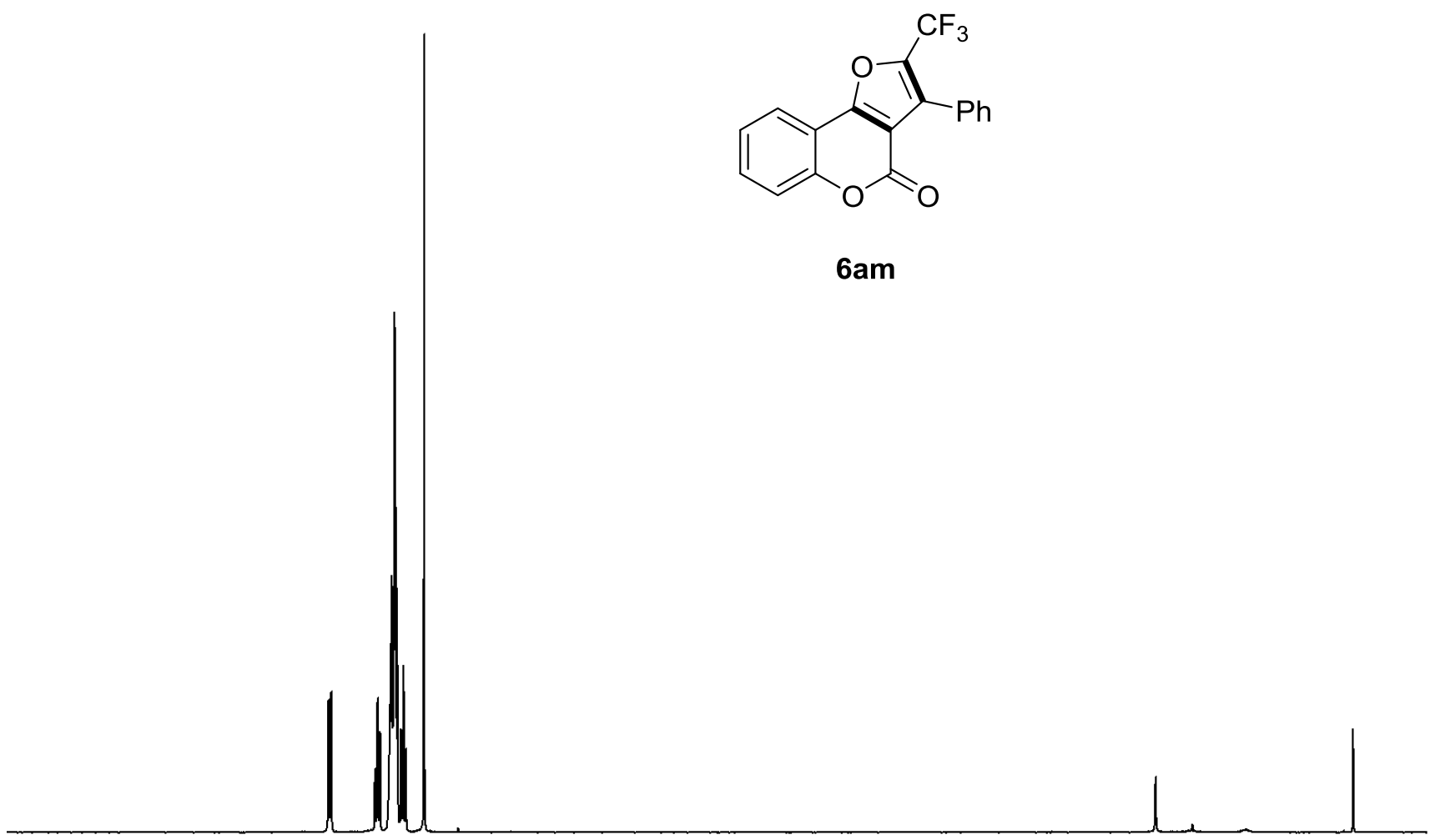

Current Data Parameters

NAME David 198 (3f)

EXPNO

ROCNO

F2 - Acquisition Parameters

Date__ 20200822

Time 23.52

INSTRUM spect

PROBHD $5 \mathrm{~mm}$ PABBO BB/

PULPROG $\quad$ zg30

SOLVENT

NS

DS

SWH

FIDRES

$\mathrm{AQ}$

$\mathrm{RG}$

$\mathrm{DW}$

DE

D1

TD0

$=====$

SEO

NUC

$\begin{array}{lr}\text { P1 } & 15.00 \\ \text { PLW1 } & 11.39999962\end{array}$

$\mathrm{zg} 30$
32768

$\mathrm{CDCl}$

0
$7211.539 \mathrm{~Hz}$

$0.220079 \mathrm{~Hz}$

$2.2719147 \mathrm{sec}$

198.09

69.333 usec

10.06 usec

$2.00000000 \mathrm{sec}$

CHANNEL $\mathrm{f} 1======$ $400.1324008 \mathrm{MHz}$

F2 - Processing parameters

SI 16384

SF $\quad 400.1300109 \mathrm{MHz}$

WDW

SSB
LB
GB

$\mathrm{PC}$

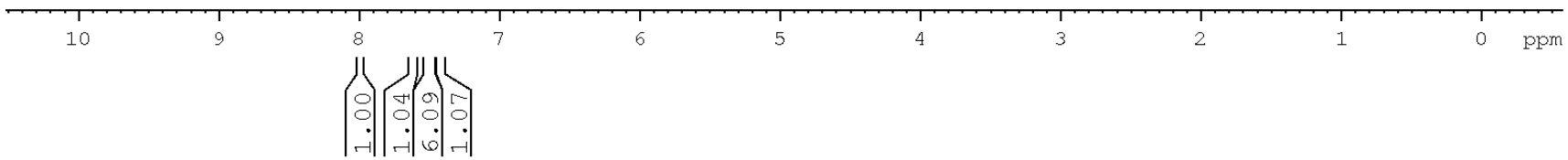


${ }^{13} \mathrm{C}$ NMR Spectrum of $6 \mathrm{am}\left(\mathrm{CDCl}_{3}, 100 \mathrm{MHz}\right)$

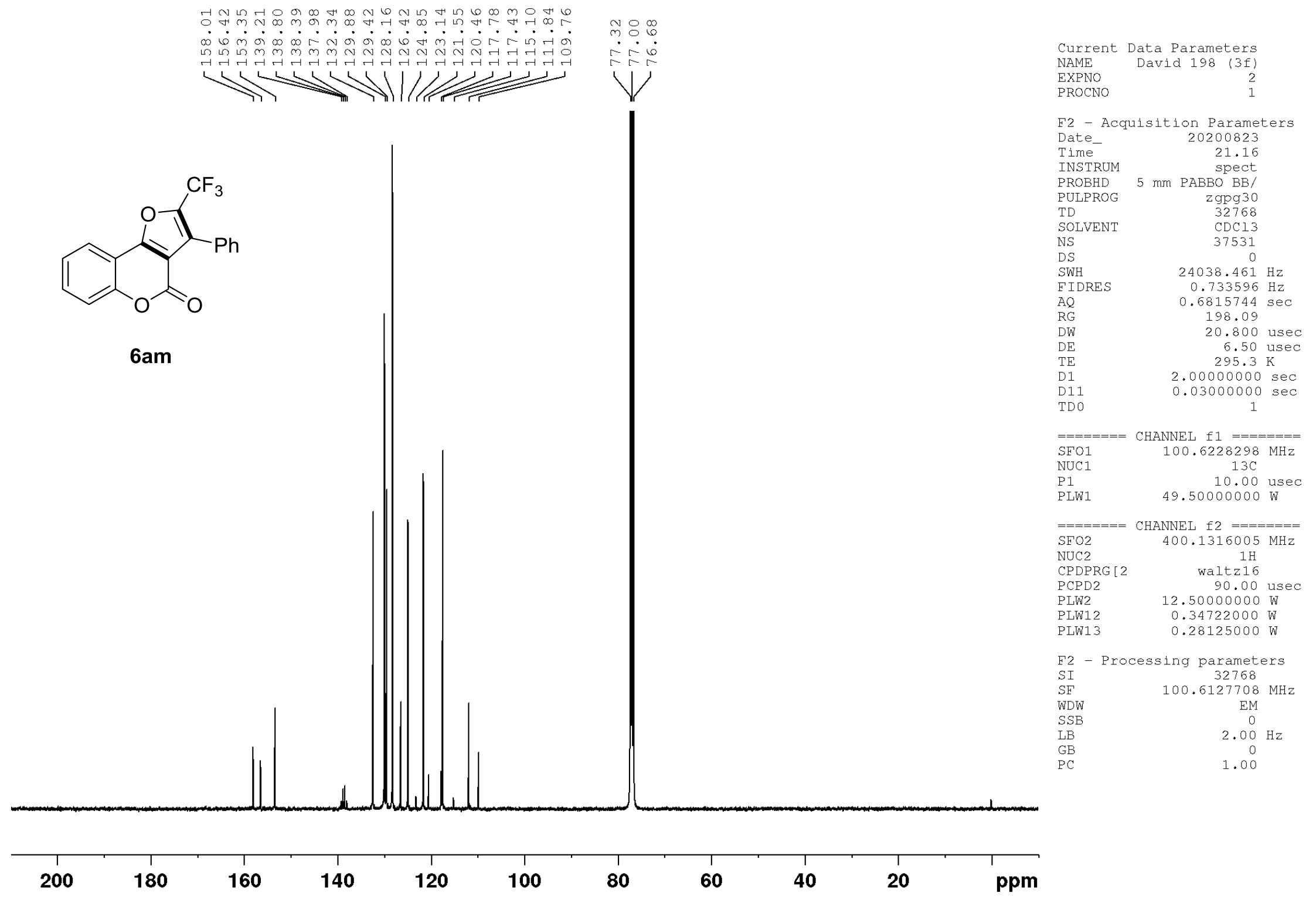


${ }^{19} \mathrm{~F}$ NMR Spectrum of $6 \mathrm{am}\left(\mathrm{CDCl}_{3}, 376 \mathrm{MHz}\right)$

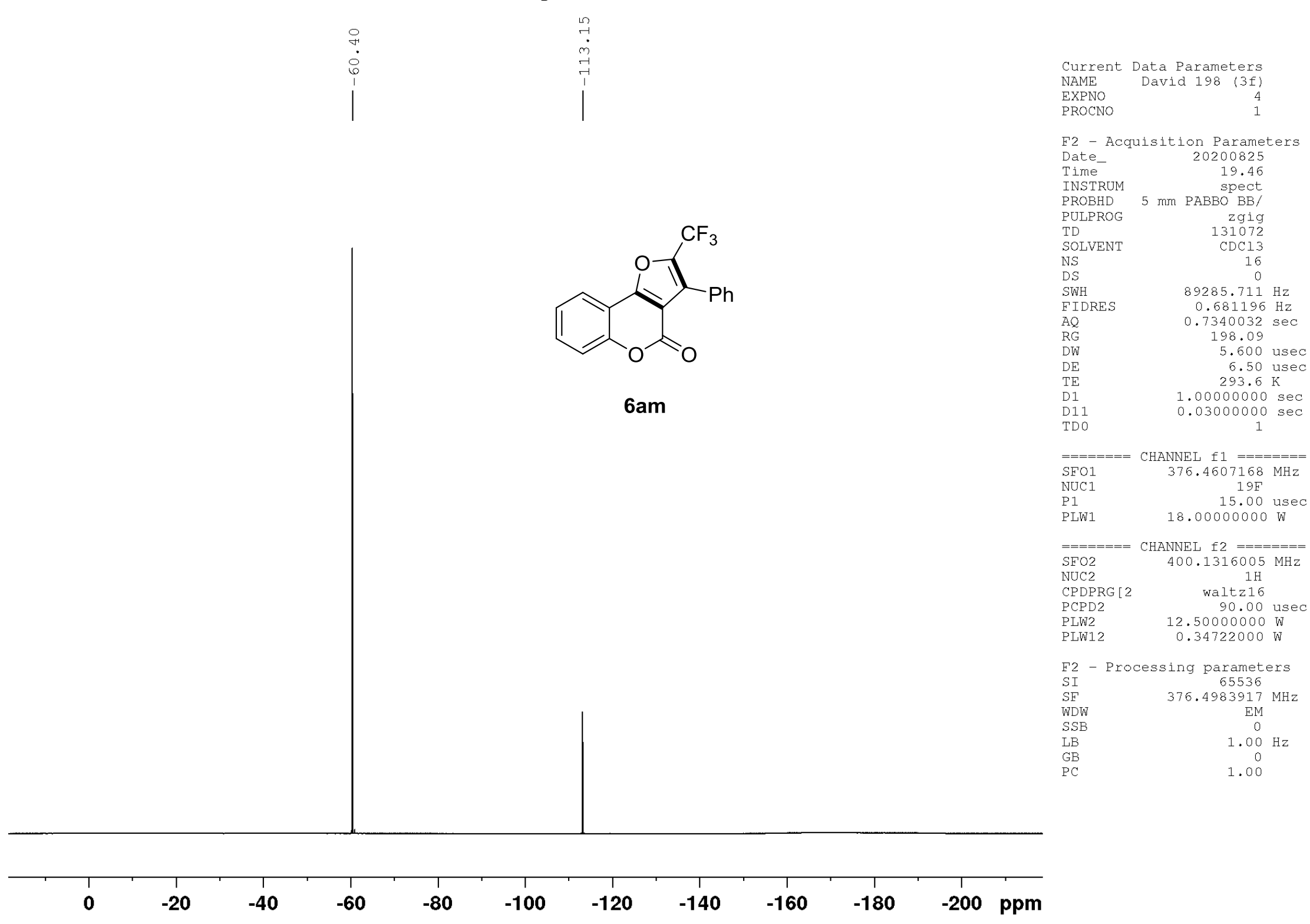


$\underline{{ }^{1} \mathrm{H} \text { NMR Spectrum of 7aa }\left(\mathrm{CDCl}_{3}, 400 \mathrm{MHz}\right)}$
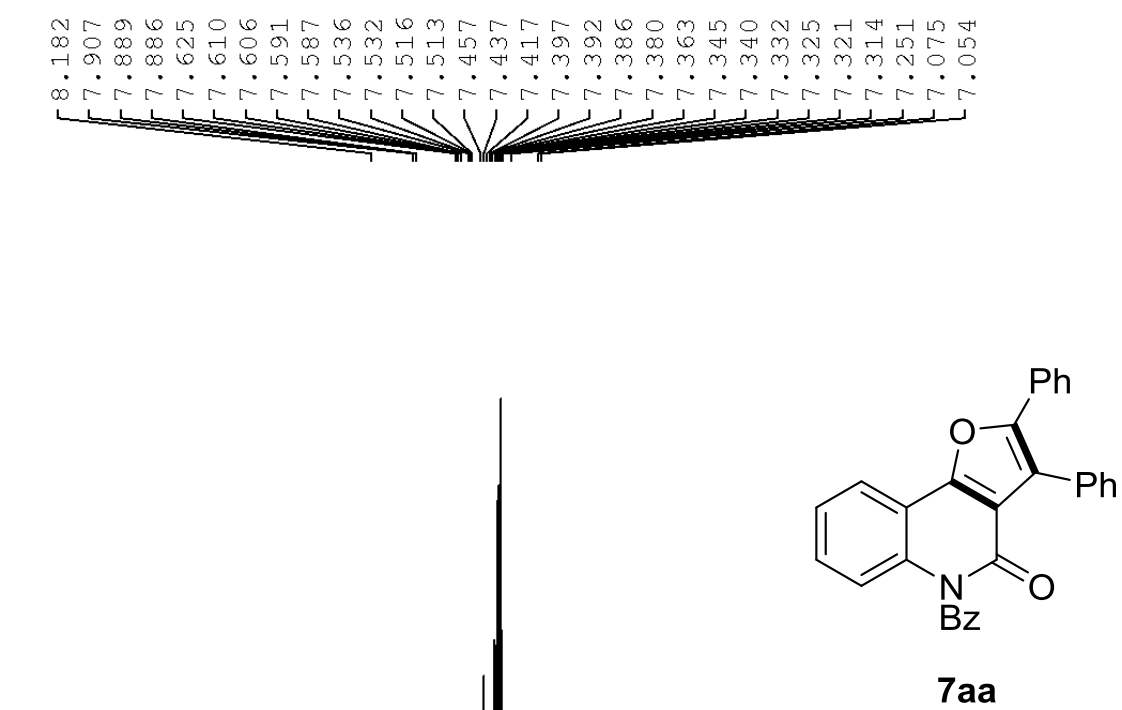

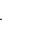

F2 - Acquisition Parameter

Date_ 20200908

Time $\quad 19.47$

INSTRUM spect

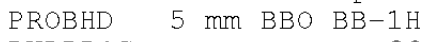

PULPROG $\quad$ zg30

TD 32768

SOLVENT CDCl3

DS

SWH $\quad 7246.377 \mathrm{~Hz}$

FIDRES $\quad 0.221142 \mathrm{~Hz}$

AQ $\quad 2.2609921 \mathrm{sec}$

RG

DW

DE
TE
D1

$.2609921 \mathrm{sec}$

69.000 usec

69.000 usec
6.50 usec $6.50 \mathrm{u}$
$297.5 \mathrm{~K}$

TDO 2.00000000 $\mathrm{sec}_{1}$

$=======$ CHANNEL $\mathrm{f} 1$

NUC1

P1

PL1

$1 \mathrm{H}$

$11.10 \mathrm{~dB}$

$400.1324008 \mathrm{MHz}$

F2 - Processing parameters

SI 16384

SF $\quad 400.1300152 \mathrm{MHz}$

WDW $\quad 400.1300152$

SSB

GB

$\mathrm{PC}$

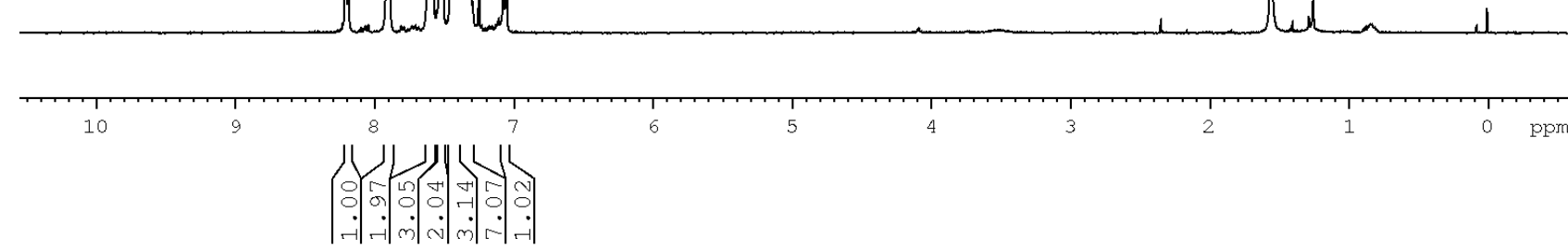


${ }^{13} \mathrm{C}$ NMR Spectrum of 7aa $\left(\mathrm{CDCl}_{3}, 100 \mathrm{MHz}\right)$

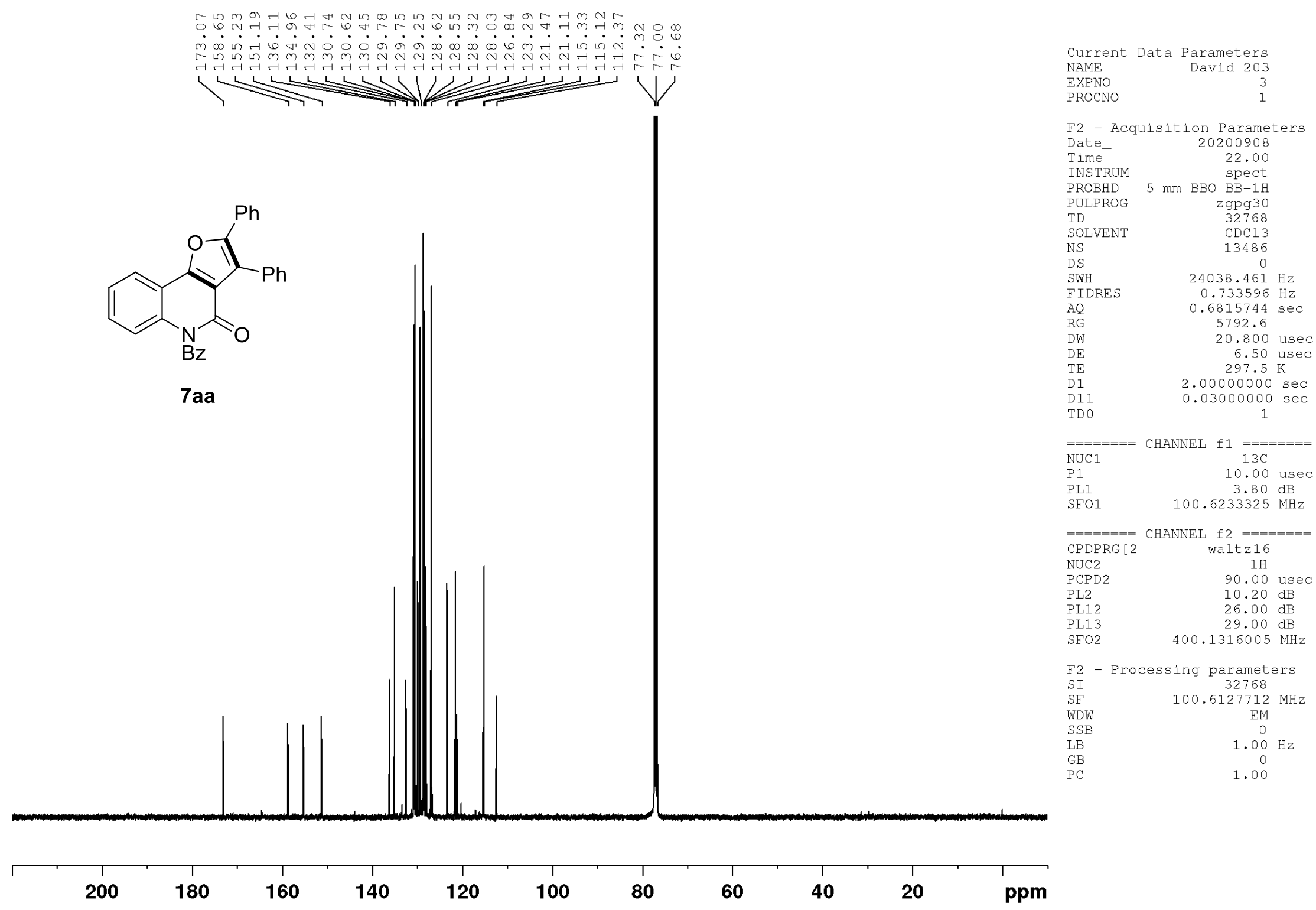


$\underline{{ }^{1} \mathrm{H} \text { NMR Spectrum of 7am }\left(\mathrm{CDCl}_{3}, 400 \mathrm{MHz}\right)}$

Current Data Parameters David 222 (3f)
1
1 (1) PROCNO

F2 - Acquisition Parameters Date__ 20200930

Time 0200930

INSTRUM

PROBHD

PULPROG mm PABBO BB/

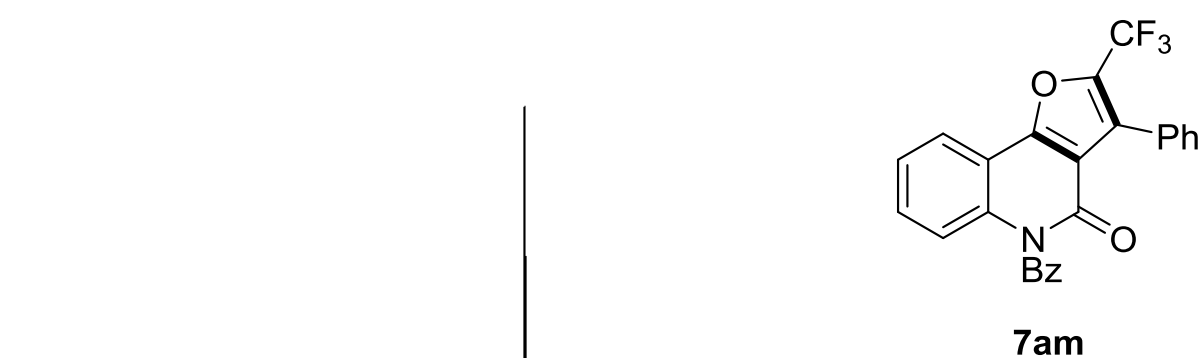

SOLVEN'T

NS

DS

SWH

FIDRES

$\mathrm{AQ}$
$\mathrm{RG}$

DW

DE

D1

TDO

$====$
SFO1

NUC $\mathrm{zg} 30$ $\mathrm{zg} 30$
32768 32

$7211.539 \mathrm{~Hz}$ $2.2719147 \mathrm{sec}$ 113.31

69.333 usec

69.333 usec
10.06 usec

10.06 use

$2.00000000 \mathrm{sec}$

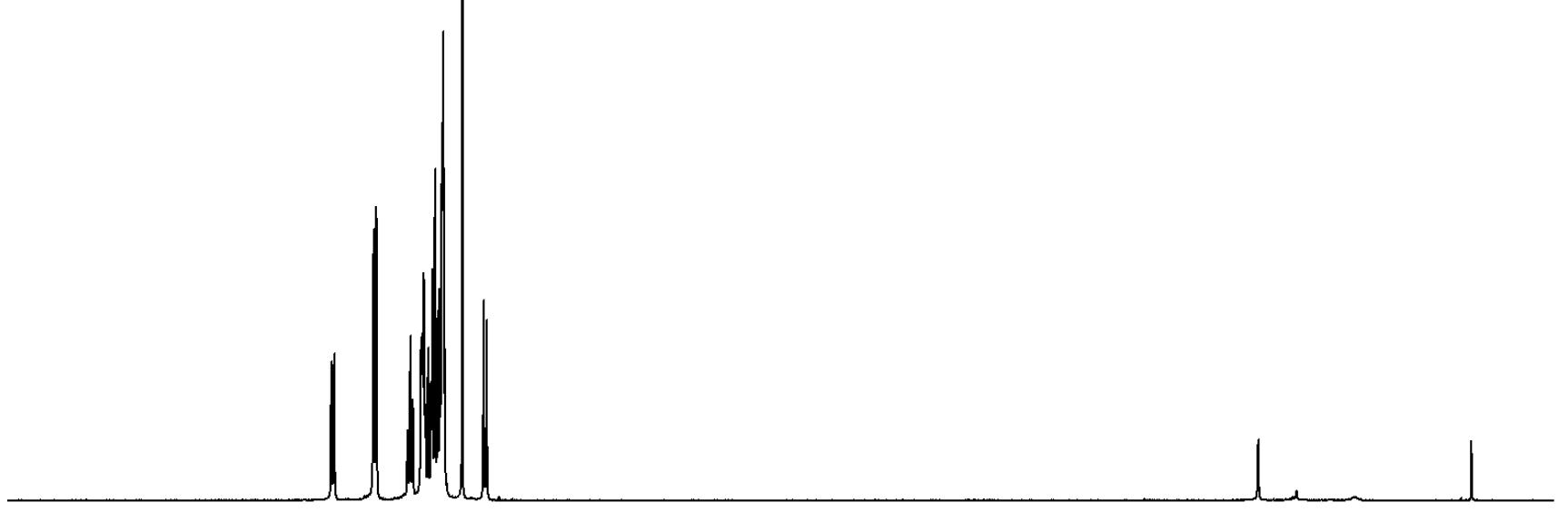

CHANNEL 11 1

LW 1

400.1324008 MHz $1 \mathrm{H}$ 15.00 usec 11.39999962 W

F2 - Processing parameters

SI 16384

SF $\quad 400.1300130 \mathrm{MHz}$

WDW

SSB
LB

LB

$\mathrm{PC}$

0

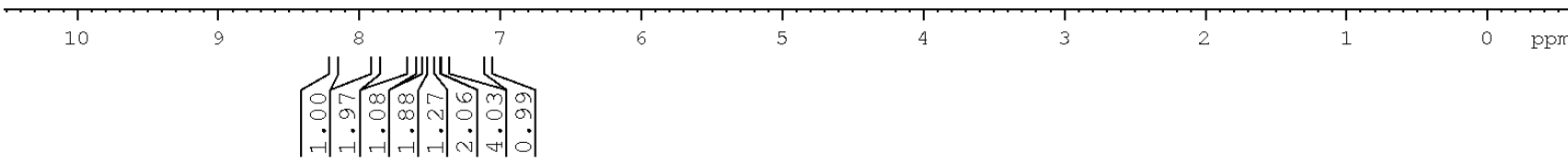


${ }^{13} \mathrm{C}$ NMR Spectrum of $7 \mathrm{am}\left(\mathrm{CDCl}_{3}, 100 \mathrm{MHz}\right)$

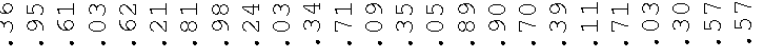

다에

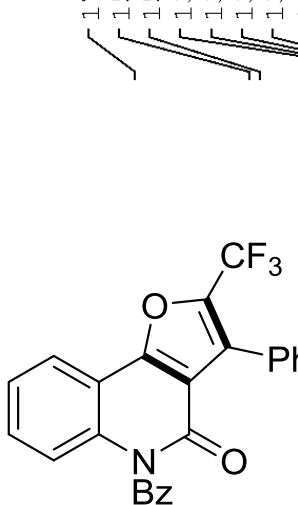

7 am

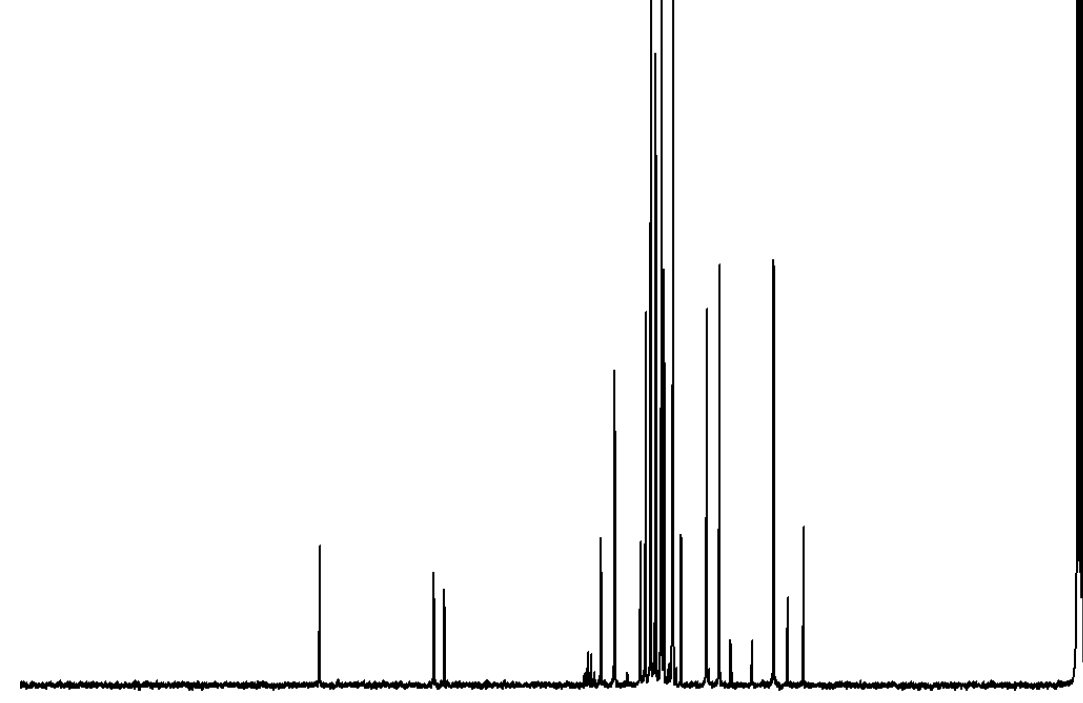

200

180

160

140

120

100

80
60

40

20

ppm

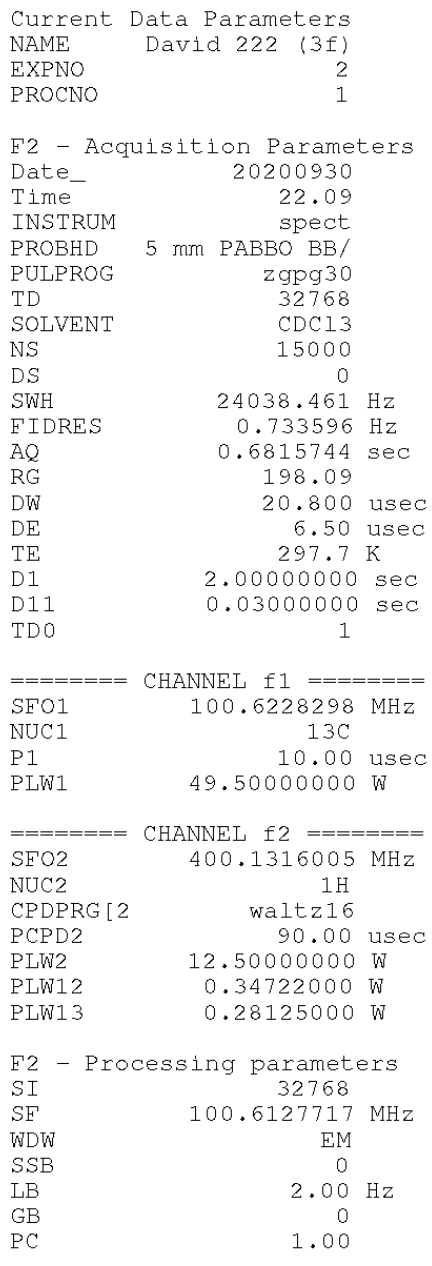


${ }^{19} \mathrm{~F}$ NMR Spectrum of $7 \mathbf{a m}\left(\mathrm{CDCl}_{3}, 376 \mathrm{MHz}\right)$

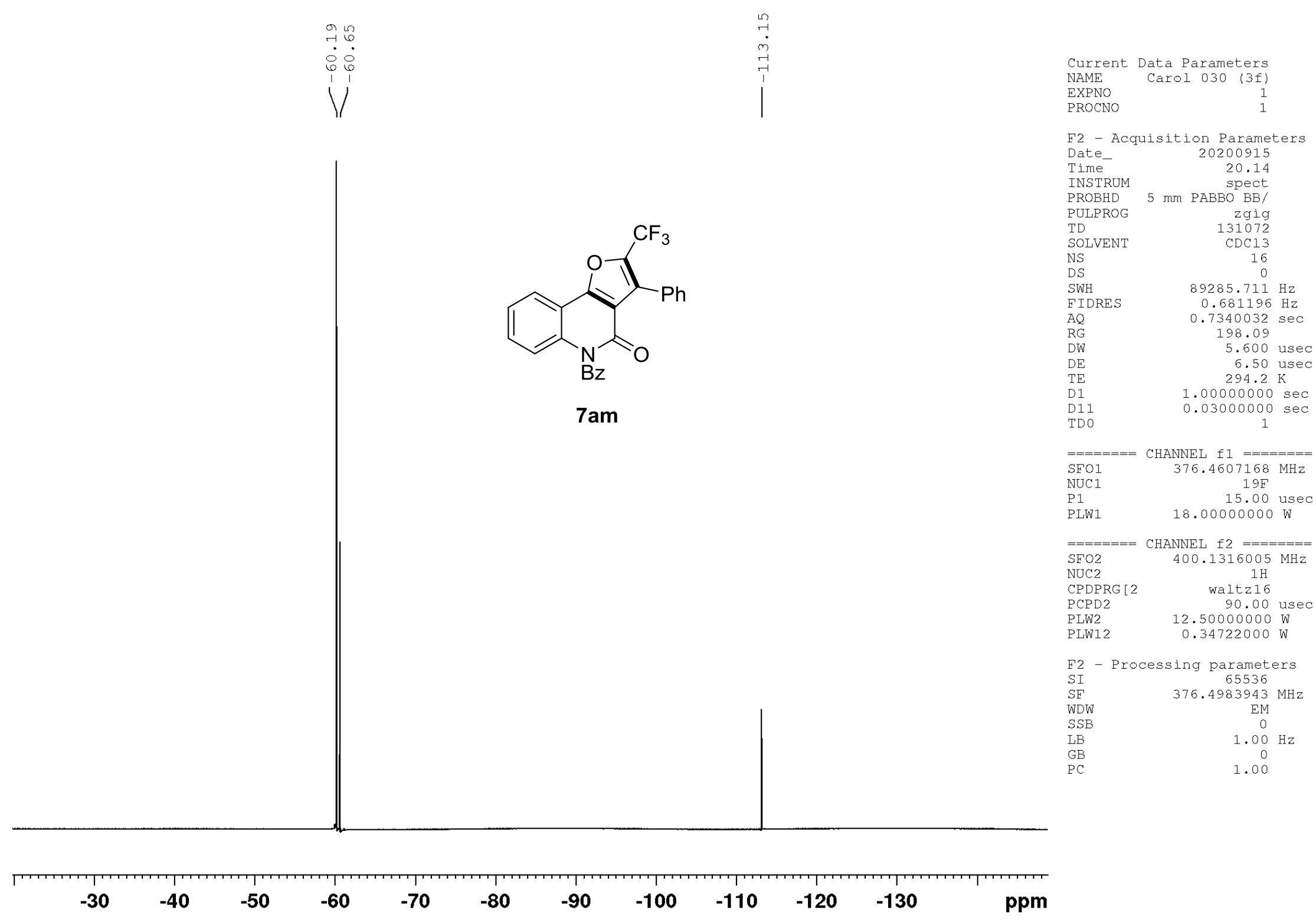


${ }^{1} \mathrm{H}$ NMR Spectrum of $\mathbf{2 a}\left(\mathrm{CDCl}_{3}, 400 \mathrm{MHz}\right)$
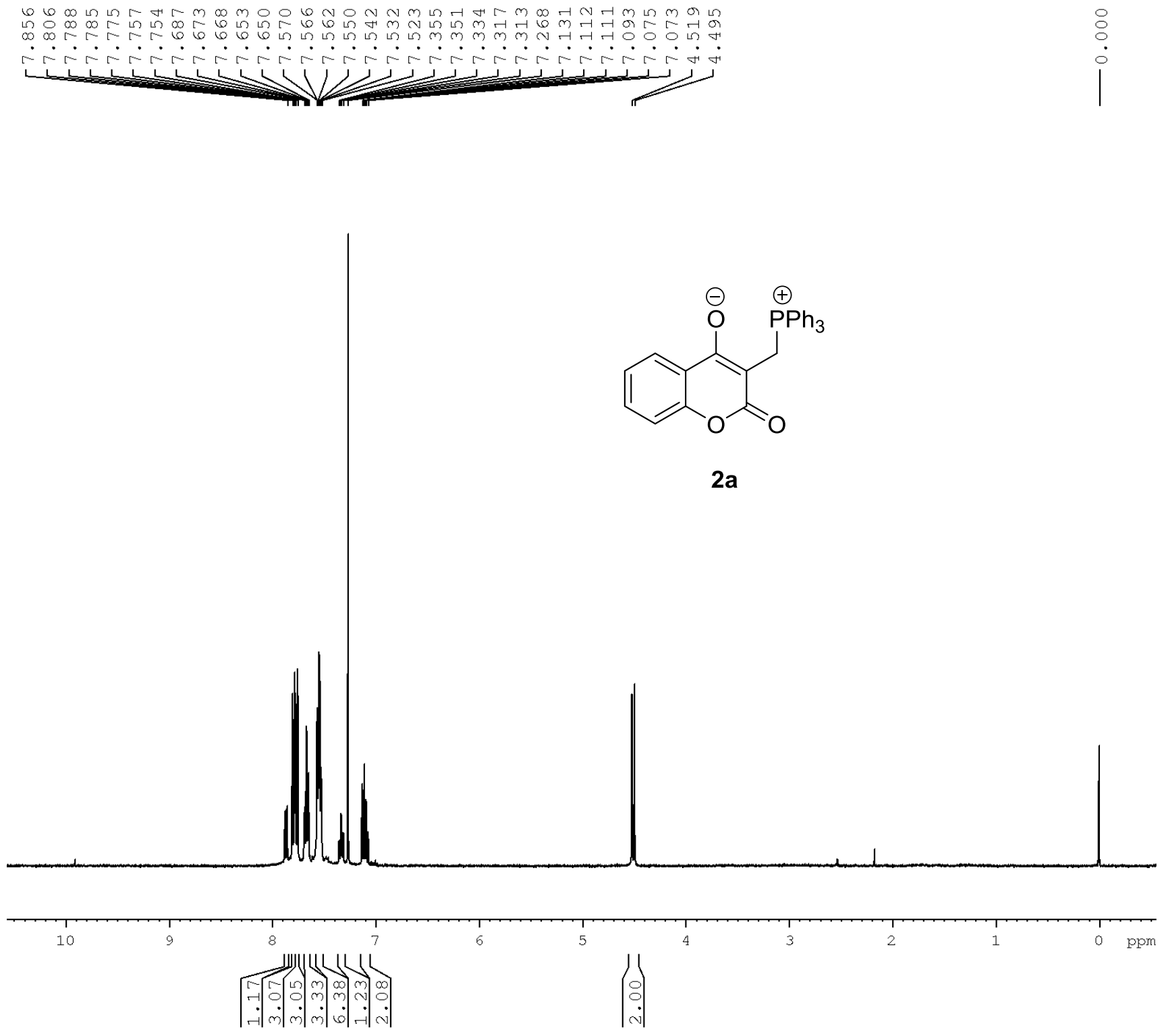

$\begin{array}{lr}\text { Current Data } & \text { Parameters } \\ \text { NAME } & \text { SW } 890-1 \\ \text { EXPNO } & 8 \\ \text { PROCNO } & 1\end{array}$

F2 - Acquisition Parameters

Date_ 20200528

Time

INSTRUM

ROBHD

ULPROG

SOLVEN

DS

SWH

FIDRES

$\mathrm{AQ}$
$\mathrm{RG}$

DW

$\mathrm{DE}$

D1

TDO

$====$

SEO

NUC

P1

$$
\begin{array}{r}
0.47 \\
\text { spect }
\end{array}
$$

$5 \mathrm{~mm}$ PABBO BB/

$\mathrm{zg} 30$
32768

CDCl3

0
$7211.539 \mathrm{~Hz}$

$0.220079 \mathrm{~Hz}$

2.2719147 sec

177.16

69.333 usec

10.06 usec

$2.00000000 \mathrm{sec}$
1

CHANNEL $\mathrm{f} 1=======$ $400.1324008 \mathrm{MHz}$

$1 \mathrm{H}$ 15.00 usec

11.39999962 W

F2 - Processing parameters

$\begin{array}{lr}\text { SI } & 16384 \\ \text { SF } & 400.1300065 \mathrm{MHz}\end{array}$

WDW

SSB

$\mathrm{AB}$

GB

400.1300065

1.00 
${ }^{13} \mathrm{C}$ NMR Spectrum of $\mathbf{2 a}\left(\mathrm{CDCl}_{3}, 100 \mathrm{MHz}\right)$

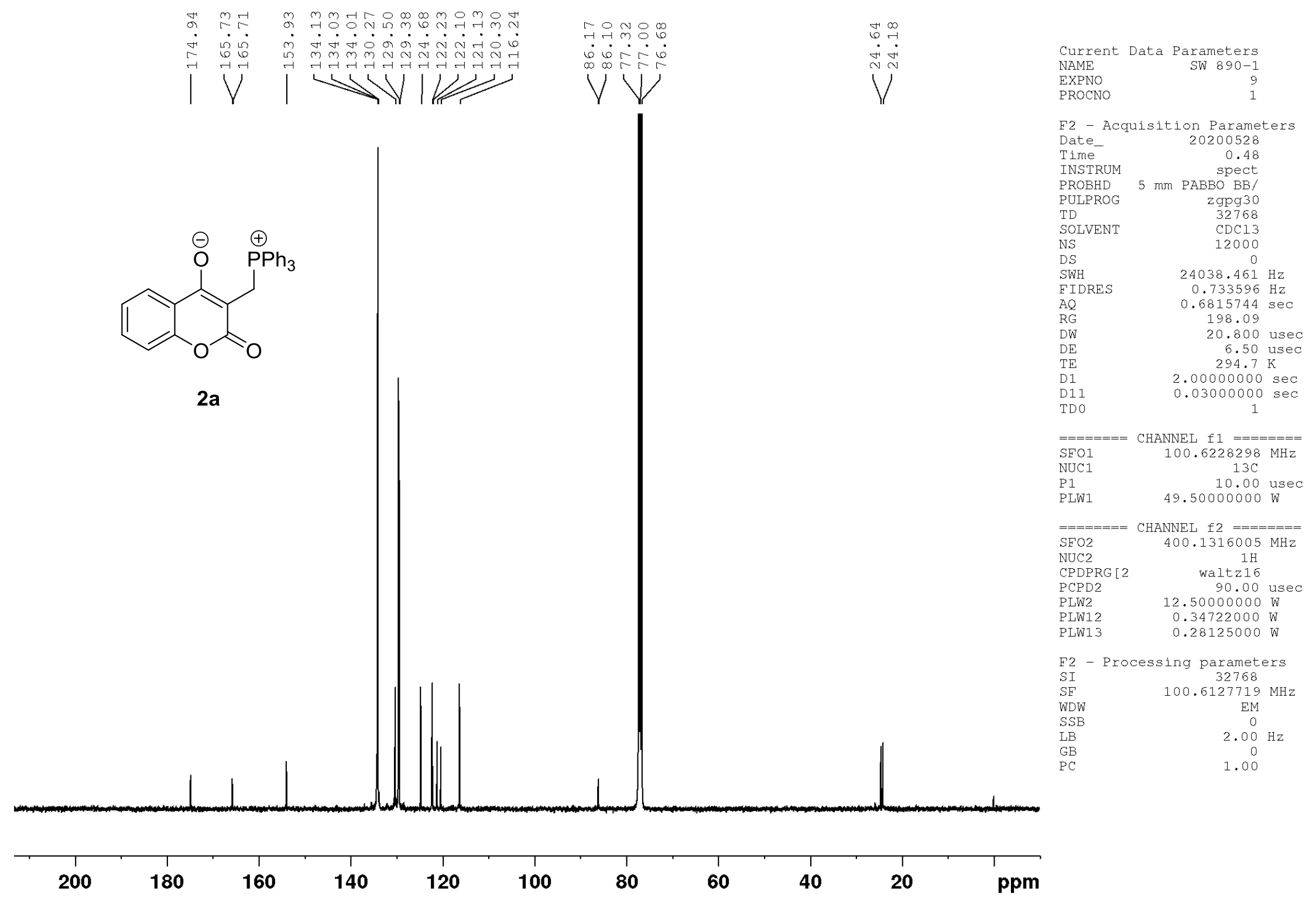


$\underline{{ }^{31} \mathrm{P} \text { NMR Spectrum of } 2 \mathbf{a}\left(\mathrm{CDCl}_{3}, 162 \mathrm{MHz}\right)}$

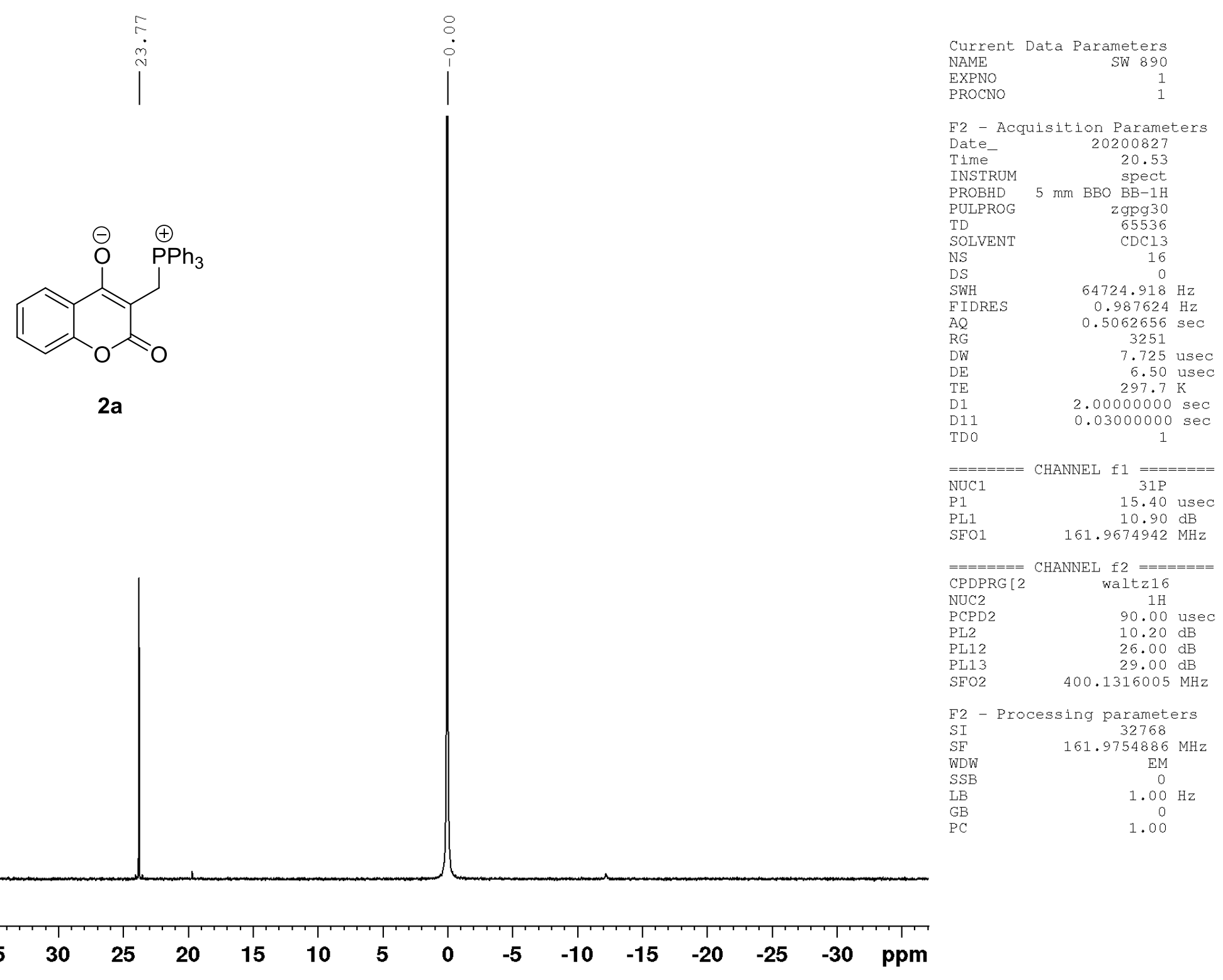


$\underline{{ }^{1} \mathrm{H} \text { NMR Spectrum of } 11 \mathrm{an}\left(\mathrm{CDCl}_{3}, 400 \mathrm{MHz}\right)}$

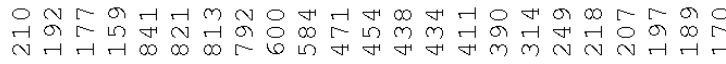

$\rightarrow$

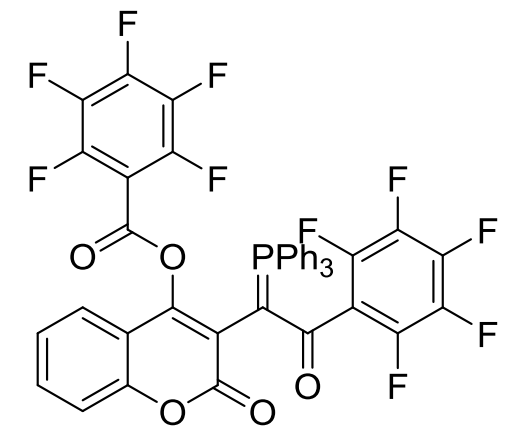

Current Data Parameters

NAME David 211 (3f)

EXPNO

PROCNO

F2 - Acquisition Parameters

Date_ 20200926

Time

INSTRUM spect

$5 \mathrm{~mm}$ spect

PULPROG

$\mathrm{BB} /$
$\mathrm{z} 930$

$\mathrm{TD}$

SOLVEN'

NS

DS

$\mathrm{SWH}$

FIDRES

$\mathrm{AQ}$
$\mathrm{RG}$
$\mathrm{DWT}$

DW

DE

D1

11 an

TD 0

$===$

$\mathrm{SEO} 1$

NUC1

P1
$\mathrm{zg} 30$
32768
$\mathrm{CDCl} 3$
0
$7211.539 \mathrm{~Hz}$
$0.220079 \mathrm{~Hz}$
$2.2719147 \mathrm{sec}$
71.42
69.333 usec
10.06 usec
$2.00000000 \mathrm{sec}$

CHANTET

$$
1
$$

15.00 usec
PLW1 $11.39999962 \mathrm{~W}$

F2 - Processing parameters

$\begin{array}{lr}\text { SI } & 16384 \\ \text { SF } & 400.1300141 \mathrm{MHz}\end{array}$

WDW

SSB

$\mathrm{HB}$

GB

400.1300141

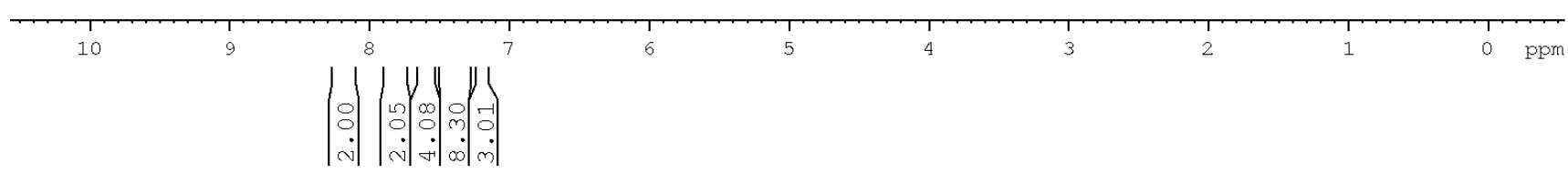


${ }^{13} \mathrm{C}$ NMR Spectrum of $11 \mathrm{an}\left(\mathrm{CDCl}_{3}, 100 \mathrm{MHz}\right)$
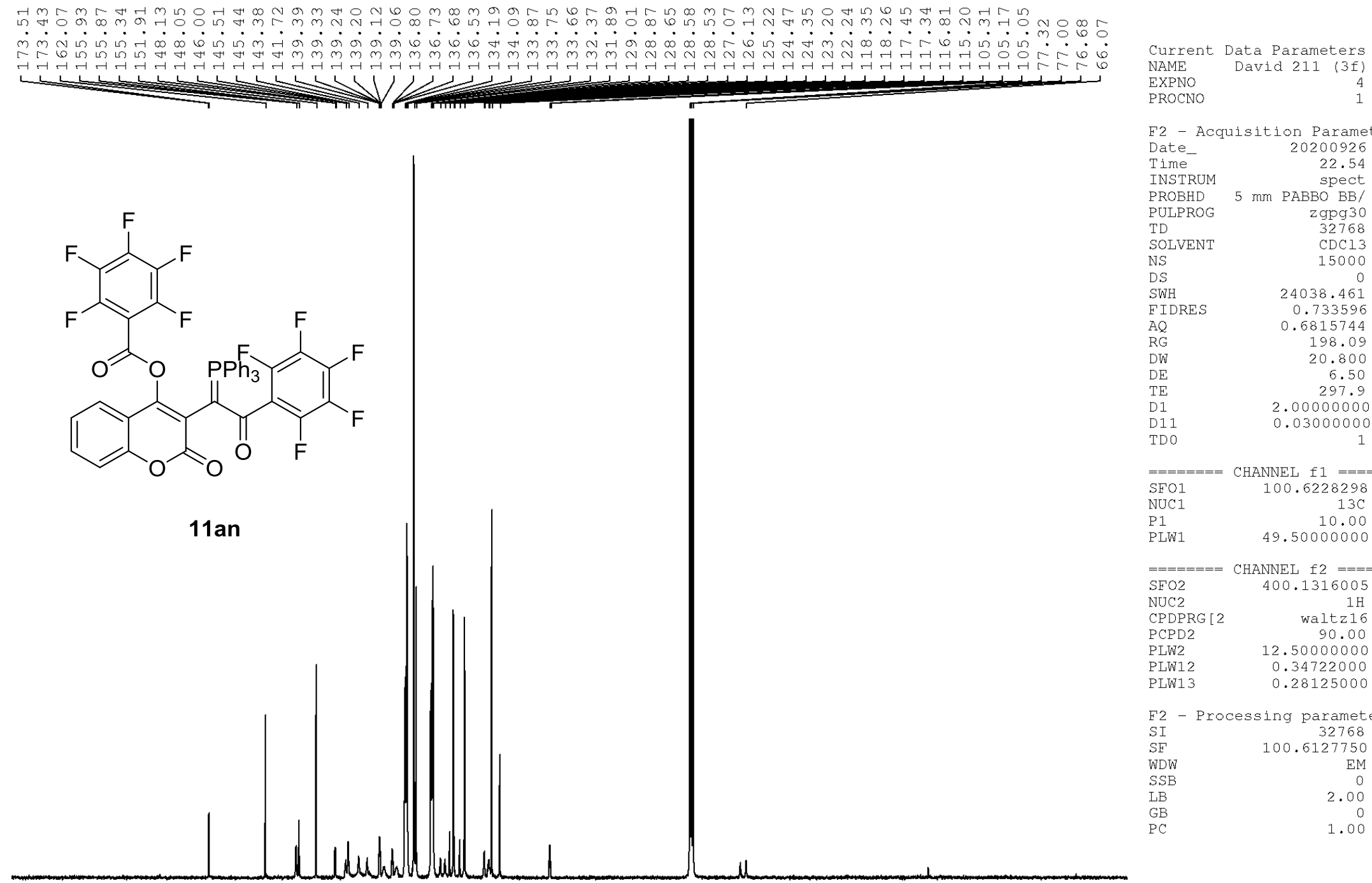

F2 - Acquisition Parameters

Date__ 20200926

Time 22.54

PROBHD $5 \mathrm{~mm}$ PABBO BB/

PULPROG
I mm PABBO

TD

DS

SWH $\quad 24038.461 \mathrm{~Hz}$

FIDRES $\quad 0.733596 \mathrm{~Hz}$

AQ $\quad 0.6815744 \mathrm{sec}$

DW

DE

198.09
20.800 usec

20.800 usec
6.50 usec

TE $\quad 297.9 \mathrm{~K}$

D11 $\quad 2.00000000 \mathrm{sec}$

TDO

$\begin{array}{ll}======= & \text { CHANNEL } \mathrm{f} 1 \quad======= \\ \text { SFO1 } & 100 \cdot 6228298 \mathrm{MHz}\end{array}$

$13 \mathrm{C}$ usec
P1 10.00 use

PLW1 49.50000000 W

$=======$ CHANNEL f2 $========$
SFO2
No0.1316005 MHz

CPDPRG [2

PCPD2

PLW12

PLW13

$1 \mathrm{H}$
000.1316005
90.00

90.00 usec

$12.50000000 \mathrm{~W}$

$0.34722000 \mathrm{~W}$

2 - Processing paraneters

SI 32768

WDW

SSB

PC

$\mathrm{EM}$
0
$2.00 \mathrm{~Hz}$
0

$2.00 \mathrm{H}$
0
1.00

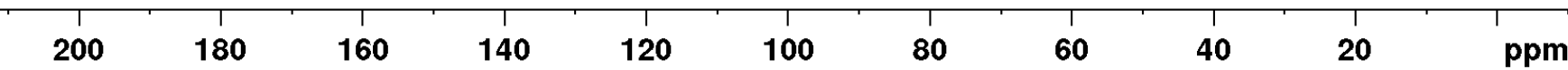


${ }^{19} \mathrm{~F}$ NMR Spectrum of $\mathbf{1 1 a n}\left(\mathrm{CDCl}_{3}, 376 \mathrm{MHz}\right)$

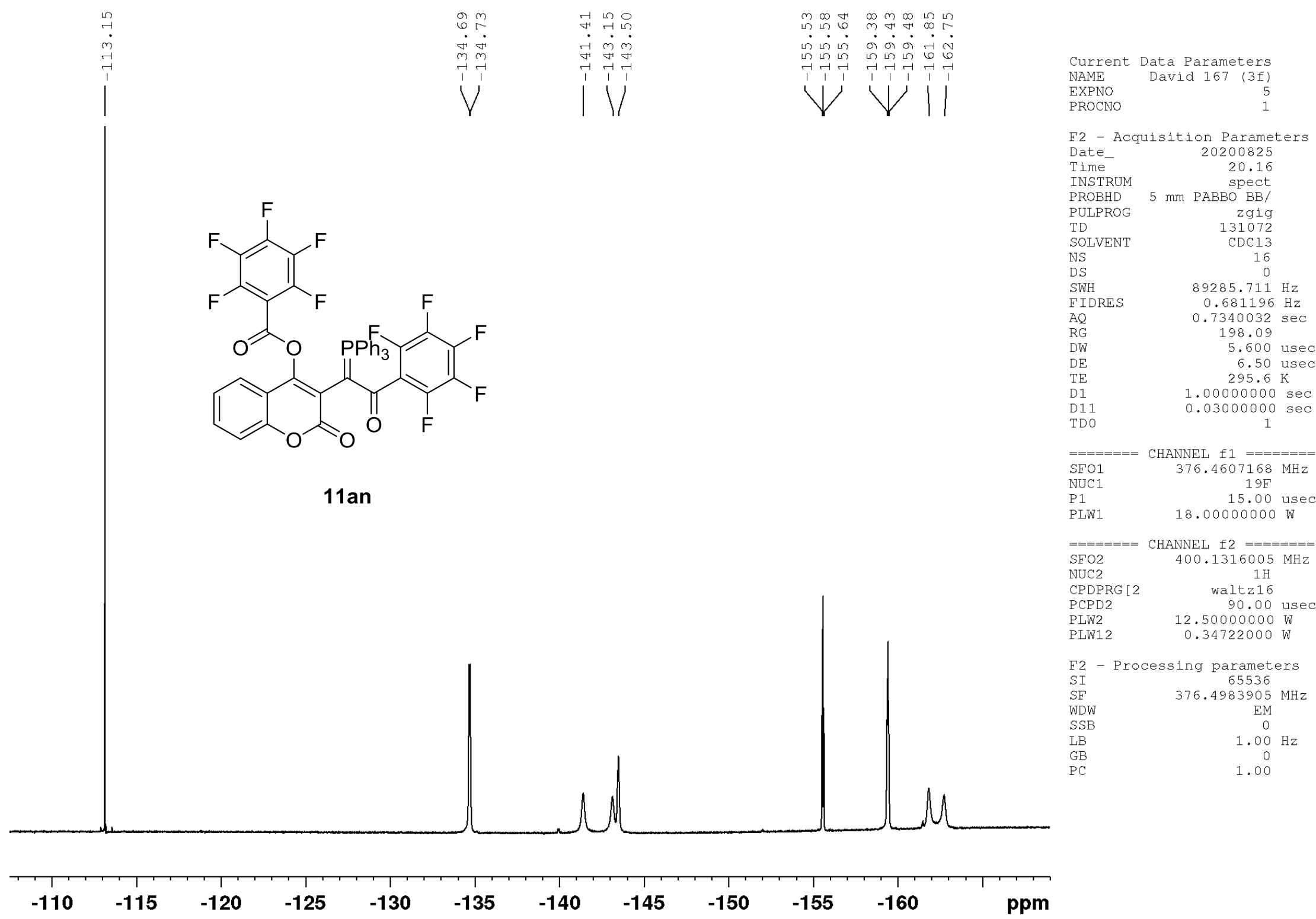


${ }^{31} \mathrm{P}$ NMR Spectrum of 11an $\left(\mathrm{CDCl}_{3}, 162 \mathrm{MHz}\right)$

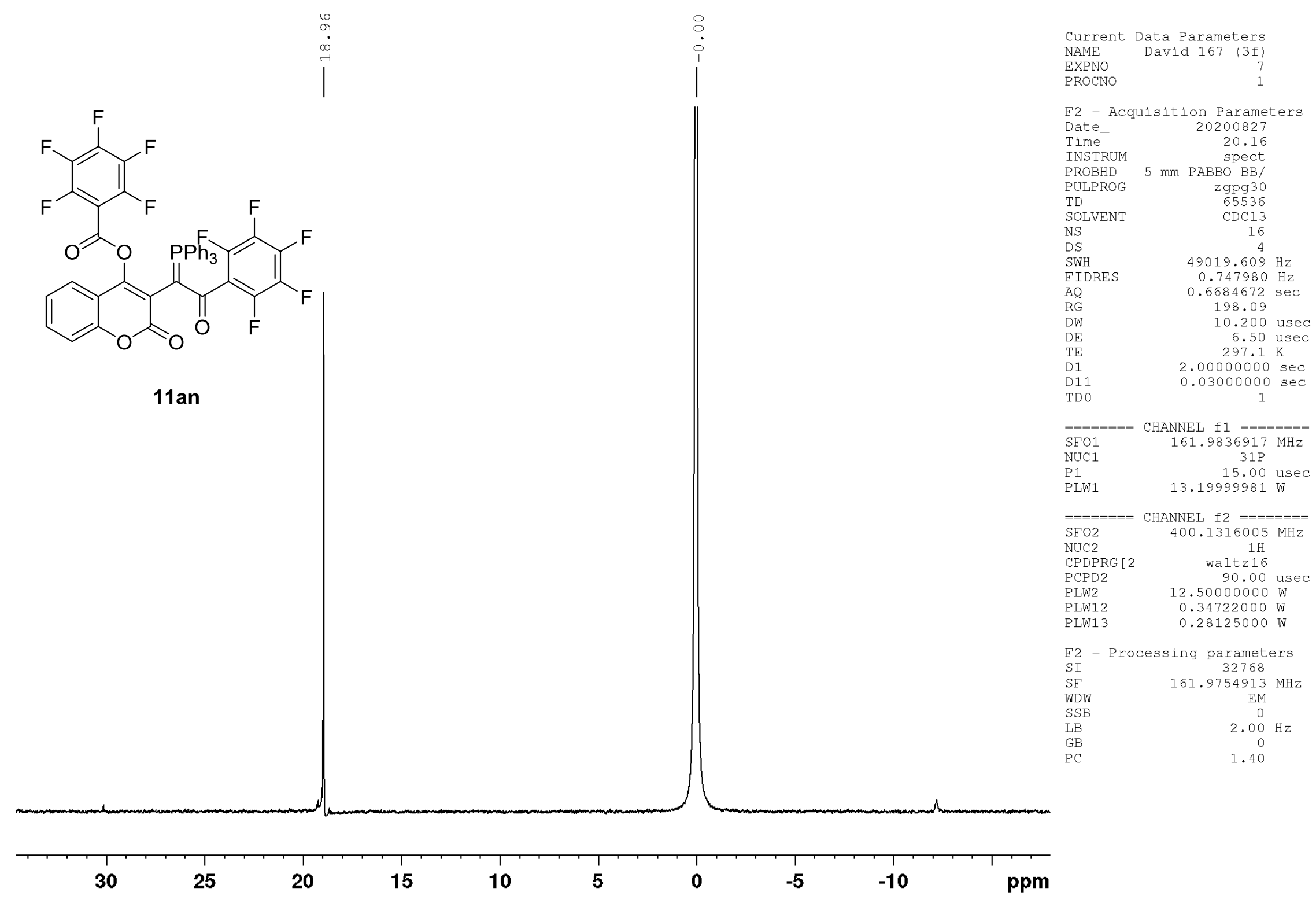

